Aus der Abteilung Medizinische Informatik

(Prof. Dr. med. O. Rienhoff)

im Zentrum Epidemiologie, Statistik und Informatik

der Medizinischen Fakultät der Universität Göttingen

\title{
Aufbau eines medizinischen Virtual-Reality-Labors und Entwicklung eines VR-gestützten neuropsychologischen Testsystems mit einer präklinischen und klinischen Evaluationsstudie
}

\author{
INAUGURAL - DISSERTATION \\ zur Erlangung des Doktorgrades \\ der Medizinischen Fakultät \\ der Georg-August-Universität zu Göttingen
}

vorgelegt von

Marcus Alfons Mehlitz

aus

Lüdinghausen/Westfalen

Göttingen 2004 
D e k a n:

I. Berichterstatter:

II. Berichterstatter/in:

III. Berichterstatter/in:
Prof. Dr. Med. M. Droese

Prof. Dr. med. O. Rienhoff

Prof. Dr. med. B. Bandelow

Tag der mündlichen Prüfung: 21. Oktober 2004 


\section{Inhaltsverzeichnis}

1. Einleitung 1

1.1. Motivation der Arbeit 1

1.2. Virtuelle Realität und ihre Anwendung in der Medizin und den Neurowissenschaften

1.2.1. Definition und Eigenschaften von Virtueller Realität 3

1.2.2. Technische Realisation von Virtueller Realität 4

1.2.2.1. Eingabesysteme (Real Environment Sensing) 4

1.2.2.2. Anzeigesysteme (Virtual Environment Display) 5

1.2.2.3. Steuer- und Kontrollsysteme (Virtual Environment Control) 7

1.2.2.3.1. Hardware 7

$\begin{array}{ll}\text { 1.2.2.3.2. Software } & 10\end{array}$

1.2.3. Unerwünschte Effekte bei der Benutzung virtueller Umgebungen 11

1.2.3.1. Definition und Pathophysiologie 11

1.2.3.2. Erfassung von Nebenwirkungen 11

1.2.3.3. Individuelle und technische Faktoren mit Einfluss auf $\begin{array}{ll}\text { Simulator-Sickness-Symptome } & 12\end{array}$

1.2.3.4. Behandlung der Nebenwirkungen 13

1.2.3.5. Probleme und offene Fragen hinsichtlich unerwünschter Nebenwirkungen 14

1.2.4. Medizinische Anwendung von VR 14

1.2.4.1. Überblick und Beispiele von Einsatzgebieten 14

1.2.4.2. VR als Werkzeug in den Neurowissenschaften 16

1.3. Neuropsychologische Grundlagen 16

1.3.1. Anwendungsgebiete neuropsychologischer Testverfahren 16

1.3.2. Neuropsychologische Funktionen mit Relevanz für Testsystem 16

1.3.3. Überblick über Virtual-Reality-gestützte Anwendungen im Bereich der Neuropsychologie 
1.4.1. Planung und Aufbau eines interdisziplinären, medizinischen VR-Labors 20

1.4.2. Entwicklung eines neuropsychologischen Testsystems 20

1.4.3. Evaluationsstudien zur Anwendung des Testsystems 21

1.4.3.1. Praktikabilität des Testsystems 22

1.4.3.2. Wirkungen virtueller Realität 22

1.4.3.3. Nebenwirkungen virtueller Realität 23

2. Methodik und Material 24

2.1. Analyse medizinischer VR-Anwendungen 24

2.2. Aufbau und Organisation des VR-Labors 25

2.3. Überblick: Testentwicklung und Evaluation 26

2.4. Entwicklung des Neuropsychologischen Testsystems 28

2.4.1. Analyse und Definition 28

2.4.1.1. Analyse und Definition: Neuropsychologische Virtual-Reality-Testumgebung 28

2.4.1.2. Analyse und Definition: EDV-gestützte Testadministration 31

2.4.1.3. Analyse und Definition: Auswertung 31

2.4.2. Testsystem-Prototyp 31

2.4.3. Entwurf 33

2.4.3.1. Entwurf Modul 1: Virtual-Reality-Testumgebung 33

2.4.3.2. Entwurf Modul 2: Testadministration 35

2.4.3.3. Entwurf Modul 3: Auswertung 36

$\begin{array}{ll}\text { 2.4.4. Implementation } & 37\end{array}$

$\begin{array}{ll}\text { 2.5. Evaluation } & 38\end{array}$

2.5.1. Erfassung erwünschter und unerwünschter VR-bedingter Effekte 38

2.5.2. Bedienbarkeit und Beurteilung des Testsystems durch die Testperson 38

2.5.3. Stichprobe, Material und Testdurchführung der präklinischen Studie 39 
2.5.3.1. Auswahl der Stichprobe 39

$\begin{array}{ll}\text { 2.5.3.2. Material } & 39\end{array}$

2.5.3.3. Testdurchführung 40

2.5.4. Stichprobe, Material und Testdurchführung der klinischen Studie 42

2.5.4.1. Auswahl der Stichprobe 42

2.5.4.2. Material 43

2.5.4.3. Testdurchführung 43

$\begin{array}{ll}\text { 2.5.5. Datenanalyse } & 44\end{array}$

3. Ergebnisse 45

3.1. Technische Analyse medizinischer Virtual-Reality-Anwendungen 45

3.1.1. Auswertung 46

$\begin{array}{ll}\text { 3.1.2. Zusammenfassung } & 48\end{array}$

3.2. Organisation und Aufbau des VR-Labors 49

3.2.1. Laborkonzept 49

3.2.2. Komponenten des eingerichteten VR-Labors 50

3.2.2.1. Entwicklungswerkzeuge 50

3.2.2.2. Steuer- und Kontrollsystem 50

3.2.2.3. Eingabesysteme 50

3.2.2.4. Anzeigesysteme 51

3.2.3. Zusammenfassung 52

3.3. Neuropsychologische VR-Anwendung 52

3.3.1. Virtual-Reality-Testumgebung 52

3.3.1.1. Technische Realisation 52

3.3.1.2. Neuropsychologische Testumgebung 53

3.3.2. Testadministration $\quad 54$

3.3.3. Auswertung $\quad 55$

3.3.4. Zusammenfassung 55 
3.4. Präklinische Evaluation des neuropsychologischen Testsystems 56

3.4.1. Messzeitpunkte und statistische Methoden 56

3.4.2. Beschreibung der Stichprobe 56

3.4.3. Benutzerevaluation: Technische Realisation des Testsystems 57

3.4.4. Ergebnisse zu unerwünschten Nebenwirkungen 58

3.4.4.1. Testabbrüche aufgrund von Nebenwirkungen 58

3.4.4.2. Simulator-Sickness-Scores im Testverlauf 59

3.4.5. Zusammenfassung 60

3.4.6. Veränderungen am Testsystem als Konsequenz der präklinischen Evaluation 61

\subsection{Klinische Evaluation des Testsystems}

\section{3}

3.5.1. Beschreibung der Stichprobe der klinischen Studie 63

3.5.2. Benutzerbewertung und Praktikabilität 64

3.5.3. Bewertung von Immersion und Realismus 66

3.5.4. Auswertung der erfassten Nebenwirkungen 68

3.5.4.1. Messzeitpunkte und statistische Methoden 68

3.5.4.2. Grundauswertung 68

3.5.4.3. Interferenzstatistische Untersuchung der Simulator-Sickness-Scores 69

3.5.4.3.1. Überprüfung des Einflusses des Alters auf SSQ-Scores 69

3.5.4.3.2. Einfluss des Messzeitpunktes auf die SSQ-Scores 69

3.5.4.3.3. Überprüfung möglicher Einflussfaktoren auf die SSQ-Scores 71

3.5.4.3.4. Untersuchung der SSQ-Scores auf Interaktionseffekte $\quad 75$

$\begin{array}{ll}\text { 3.5.5. Zusammenfassung } & 77\end{array}$ 
4. Diskussion

4.1. Planung und Aufbau eines interdisziplinär nutzbaren, medizinischen VR-Labors

\subsection{Technische Entwicklung eines VR-gestützten neuropsychologischen Test-Systems}

\subsection{Präklinische und klinische Evaluationsstudie}

4.3.1. Praktikabilität des Testsystems

4.3.2. Benutzerbewertung von Realismus und Immersion

\section{Zusammenfassung}

6. Anhang

6.1. Verzeichnis der verwendeten Abkürzungen 94

6.2. Simulator Sickness Questionnaire 95

6.3. Berechnung der Scores für den Simulator Sickness Questionnaire 96

6.4. Präklinische Studie: Prä-Test-Fragebogen 97

6.5. Präklinische Studie: Post-Test-Fragebogen 99

$\begin{array}{ll}\text { 6.6. Klinische Studie: Nachbefragung } & 102\end{array}$

6.7. Erläuterungen für gesunde Kontrollprobanden 104

$\begin{array}{ll}\text { 6.8. Erläuterung für Patienten } & 105\end{array}$

6.9. Einverständniserklärung für gesunde Kontrollprobanden 106

$\begin{array}{ll}\text { 6.10. Anleitung zur Testdurchführung } & 107\end{array}$

6.11. Bildschirmmasken des Testadministrationsmoduls 112

6.12. Beispiel eines Wegenetzes der Testumgebungen 115

6.13. Bildwiederholungsrate komplexer VR-Umgebungen 115

$\begin{array}{ll}\text { 7. Literaturverzeichnis } & 116\end{array}$ 


\section{Verzeichnis der Tabellen und Abbildungen}

\begin{tabular}{|c|c|c|c|}
\hline Abb. 1.1 & 1 & Tab. 1.1 & 8 \\
\hline Abb. 1.2. & 4 & Tab. 1.2. & 9 \\
\hline Abb. 1.3. & 6 & Tab. 1.3. & 12 \\
\hline Abb. 1.4 & 6 & Tab. 1.4 & 14 \\
\hline Abb. 1.5 & 10 & Tab. 1.5 & 15 \\
\hline Abb. 1.6. & 18 & Tab. 2.1. & 37 \\
\hline Abb. 1.7 & 18 & Tab. 2.2. & 37 \\
\hline Abb. 2.1 & 27 & Tab. 3.1 & 46 \\
\hline Abb. 2.2. & 34 & Tab. 3.2. & 46 \\
\hline Abb. 2.3. & 36 & Tab. 3.3. & 47 \\
\hline Abb. 2.4 & 41 & Tab. 3.4 & 47 \\
\hline Abb. 2.5 & 44 & Tab. 3.5. & 48 \\
\hline Abb. 3.1 & 49 & Tab. 3.6. & 57 \\
\hline Abb. 3.2. & 51 & Tab. 3.7. & 59 \\
\hline Abb. 3.3. & 53 & Tab. 3.8. & 60 \\
\hline Abb. 3.4 . & 54 & Tab. 3.9. & 63 \\
\hline Abb. 3.5. & 54 & Tab. 3.10. & 64 \\
\hline Abb. 3.6. & 54 & Tab. 3.11. & 65 \\
\hline Abb. 3.7. & 54 & Tab. 3.12. & 65 \\
\hline Abb. 3.8. & 62 & Tab. 3.13. & 67 \\
\hline Abb. 3.9. & 62 & Tab. 3.14. & 67 \\
\hline Abb. 3.10. & 62 & Tab. 3.15. & 69 \\
\hline Abb. 3.11 & 62 & Tab. 3.16. & 70 \\
\hline Abb. 3.12 . & 62 & Tab. 3.17. & 70 \\
\hline Abb. 6.1 & 112 & Tab. 3.18. & 71 \\
\hline Abb. 6.2. & 112 & Tab. 3.19. & 73 \\
\hline Abb. 6.3. & 113 & Tab. 3.20. & 74 \\
\hline Abb. 6.4 & 114 & Tab. 3.21. & 74 \\
\hline Abb. 6.5 . & 114 & Tab. 3.22. & 75 \\
\hline \multirow[t]{2}{*}{ Abb. 6.6. } & 115 & Tab. 3.23. & 77 \\
\hline & & Tab. 6.1 & 15 \\
\hline
\end{tabular}




\section{Einleitung}

\subsection{Motivation der Arbeit}

'Virtuelle Realität' (virtuell, engl. virtual = scheinbar) wird häufig als eine Schlüsseltechnologie bezeichnet, für die vielfältige Anwendungsszenarien in Technik, Naturwissenschaften und Unterhaltungsindustrie entworfen wurden (Pimentel und Teixeira 1995). Der Zweck dieser Technologie ist es, realistische Simulationen von Prozessen, Objekten und Systemen zu schaffen, mit denen ein Mensch interagieren kann, kurz: computergenerierte künstliche Welten. Virtuelle Realität (VR) bietet im medizinischen Bereich vielversprechende Forschungs- und Anwendungsansätze (Bullinger und Bauer 1994, Székely und Satava 1999).

Hürden für die Verbreitung der VR-Technologie waren lange Zeit vor allem die hohen Geräteund Entwicklungskosten. Die notwendigen Investitionen in mehrstelliger Millionenhöhe erlaubten es noch bis Anfang der Neunzigerjahre nur wenigen, vornehmlich industriell orientierten Einrichtungen in Deutschland, im Bereich der Virtuellen Realität zu arbeiten (z.B. Fraunhofer-Institut für Graphische Datenverarbeitung, Darmstadt). Leistungssprünge bei Personalcomputern (PC) und die Verfügbarkeit von kommerziellen Entwicklungssystemen haben den Zugang zur VR-Technologie in den letzten Jahren erheblich erleichtert, eine Schlüsselrolle spielte dabei die Unterhaltungsindustrie (Wann und Mon-Williams 1996).

Ein Beispiel für medizinische VR-Anwendungen sind sogenannte virtuelle Endoskopien (Ferrucci 2001): aus Computertomographien (CT) oder Magnetresonanztomographien (MRT) des Patienten werden dreidimensionale Modelle eines Organsystems am Computer geschaffen. Statt durch Einführen eines Endoskopes z.B. das Colon eines Menschen zu inspizieren, kann nun der Arzt das aus Schichtaufnahmen rekonstruierte 'virtuelle Colon' untersuchen (Abb. 1.1.). Ein Beispiel aus einem anderen Bereich der Medizin ist die Simulation

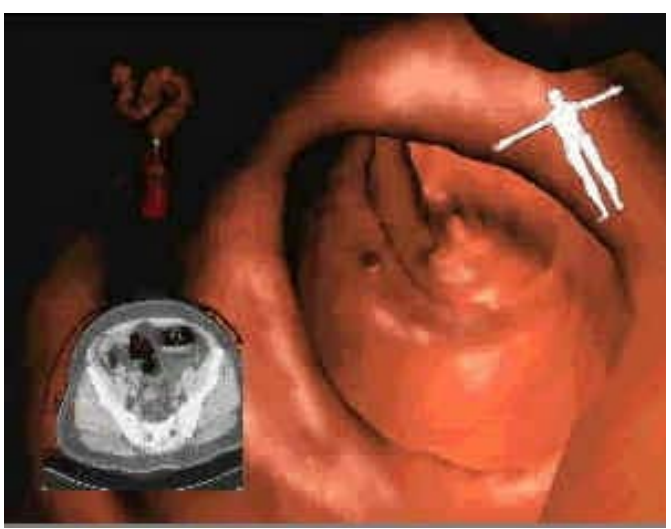

Abb. 1.1. Virtuelle Coloskopie, Daten rekonstruiert aus CTAufnahmen (Robb 1996) interaktiver Situationen zur Therapie von Angsterkrankungen (Rothbaum et. al. 1995). Bei der Verhaltenstherapie können die angsterzeugenden Stimuli mittels computergenerierter Situationen sehr variabel erzeugt werden, z.B. unterschiedlich hohe Gebäude und Aussichtspunkte bei der Therapie der Höhenangst. 
Trotz der inhaltlichen Unterschiede beider Beispiele medizinischer VR-Anwendungen basieren diese auf einem gemeinsamen technischen Prinzip: es handelt sich um interaktive, graphische Simulationen.

Um eine Forschungs- und Entwicklungsplattform für verschiedenste VR-Anwendungsgebiete am Universitätsklinikum Göttingen zu schaffen, wurde seit 1995 in der Abteilung Medizinische Informatik das Thema 'Virtuelle Realität' mit dem Ziel der Einrichtung eines interdisziplinären VR-Labors bearbeitet: durch die Bereitstellung von technischen und personellen Mitteln sollte klinischen Kooperationspartnern die Möglichkeit gegeben werden, VR-Anwendungen ohne eigene vertiefte technische Kenntnisse entwickeln zu können. Durch diese Art des Ressourcensharing sollten Anschaffungs- und Betriebskosten niedrig gehalten werden.

Bei der Planung des VR-Labors stand zunächst die Bestimmung einer notwendigen Grundausstattung, mit der sowohl psychiatrische wie chirurgische Anwendungen entwickelt werden konnten, im Vordergrund. Ein weiterer Arbeitsschwerpunkt lag aufgrund ethischer Bedenken beim Einsatz der recht jungen und undokumentierten VR-Technologie in der Medizin im Bereich Patientensicherheit und Verträglichkeit (Anonymous 1991, Whalley 1995).

Nach der Einrichtung des VR-Labors sollte im Rahmen eines Demonstrationsprojektes eine klinische Anwendung entwickelt werden. Durch Einsatz und Evaluation der Anwendung sollten Rückschlüsse auf das Laborkonzept, medizinethische Probleme und die Verwendung bestimmter VR-Technologien gewonnen werden.

Das erste klinische Kooperationsprojekt wurde durch die Abteilungen Medizinische Informatik und Psychiatrie (Schwerpunkt Neuropsychologie) der Universität Göttingen durchgeführt.

Um die Validität von Testverfahren zur Erfassung von Hirnleistungen zu verbessern, werden in der Neuropsychologie realistische Aufgabensituationen für notwendig erachtet (Buckwalter und Rizzo 1997). Exekutive und räumlich-topographische Funktionen sind Beispiele für Hirnleistungen, deren Operationalisierung aufgrund des geringen Realitätsgrades der verfügbaren Paper \& Pencil-Testverfahren als unzureichend betrachtet wird (Lezak 1995). Hier können möglicherweise durch virtuelle Realität realistischere und damit validere Testverfahren entwickelt werden. 


\subsection{Virtuelle Realität und ihre Anwendung in der Medizin und den Neurowissenschaften}

\subsubsection{Definition und Eigenschaften von Virtueller Realität}

Unter 'Virtueller Realität' (VR) versteht man Mensch-Computer-Schnittstellen, die es dem Benutzer erlauben, mit computergenerierten Umgebungen und Situationen zu interagieren und diese realitätsnah zu erleben (Pimentel und Teixeira 1995).

Eine wirklichkeitsnahe Darstellung und die realitätsnahe Einbindung und Interaktionsfähigkeit des Benutzers sind Schlüsselelemente der VR. Virtuelle Realität als 'die derzeit am weitesten entwickelte Technologie im Bereich der Mensch-Computer-Interaktion' (Kaltenborn und Rienhoff 1993, S. 407/8) besitzt gemeinsame Merkmale mit anderen Computertechniken, wie z.B. dreidimensionaler Simulation oder Multimedia. Um eine Abgrenzung zu verwandten Technologien zu ermöglichen, werden bei 'Virtueller Realität' drei wesentliche Eigenschaften gefordert (Kaltenborn und Rienhoff 1993):

1. Eine realistische Repräsentation der künstlichen Umgebung durch den Computer anhand visueller, evtl. zusätzlich auditiver oder taktiler Techniken.

2. Eine Interaktion in Echtzeit, d.h. zwischen einer Handlung des Benutzers und der daraus folgenden Veränderung der virtuellen Umgebung (z.B. Bewegung) sollten keine wahrnehmbaren Verzögerungen auftreten.

3. Verwendung von am Menschen orientierten Interaktionsmöglichkeiten, die im Gegensatz zu Tastatur und Maus natürliche Bewegungen und Kommunikationswege wie Sprache und Gestik erlauben.

Die oben genannten Forderungen nach einer realistischen Repräsentation, einer verzögerungsfreien und natürlichen Interaktion sind Idealvorstellungen für VR-Anwendungen. Diese müssen einerseits dem jeweiligen Stand der Technik als auch der Anwendungssituation entsprechend angepasst werden. Technische Realisationsmöglichkeiten werden weiter unten dargestellt (Kap. 1.2.2.).

Neben der technisch orientierten Definition von Virtueller Realität sind subjektive Kriterien eingeführt worden, mit denen Charakteristika von VR beschrieben werden sollen: das $z$. Zt. wichtigste Konzept, das zur Beschreibung von VR-induzierten Zuständen existiert, ist der 'Sense of Presence' (Sheridan 1992, Slater et al. 1994). Der 'Sense of Presence' (SOP) ist ein mehrdimensionales Konstrukt, das folgende Bewertungen des Benutzers beinhaltet:

- Inwieweit fühlt sich der Benutzer in die virtuelle Umgebung hineinversetzt (= 'Immersion', engl. to immerse $=$ eintauchen, sich vertiefen in) ? 
- Inwieweit fühlt sich der Benutzer befähigt, mit der virtuellen Umgebung zu interagieren ?

- Wie stark ist die Aufmerksamkeit des Benutzers auf die Interaktion mit der virtuellen Umgebung gerichtet?

Von Witmer und Singer wurde ein Fragebogen mit 32 Items zur Erhebung von Faktoren des SOP entwickelt (Witmer und Singer 1998). Der SOP wird auch als subjektives Maß zur qualitativen Bewertung von technischen Realisationen von VR-Anwendungen herangezogen (z.B. Hendrix und Barfield 1996, Welch et al. 1996).

\subsubsection{Technische Realisation von Virtueller Realität}

Grimsdale (1992) unterscheidet folgende Teilsysteme einer VR-Realisation: Systeme zur Erfassung von Zuständen der wirklichen Umgebung (eng/. real environment sensing), die Systemsteuerung zur Verarbeitung (Eingabe, Ausgabe) der Daten (eng/. virtual environment control), und Anzeigesysteme (engl. virtual environment display), die in ihrem Zusammenspiel in Abb. 1.2. dargestellt sind.

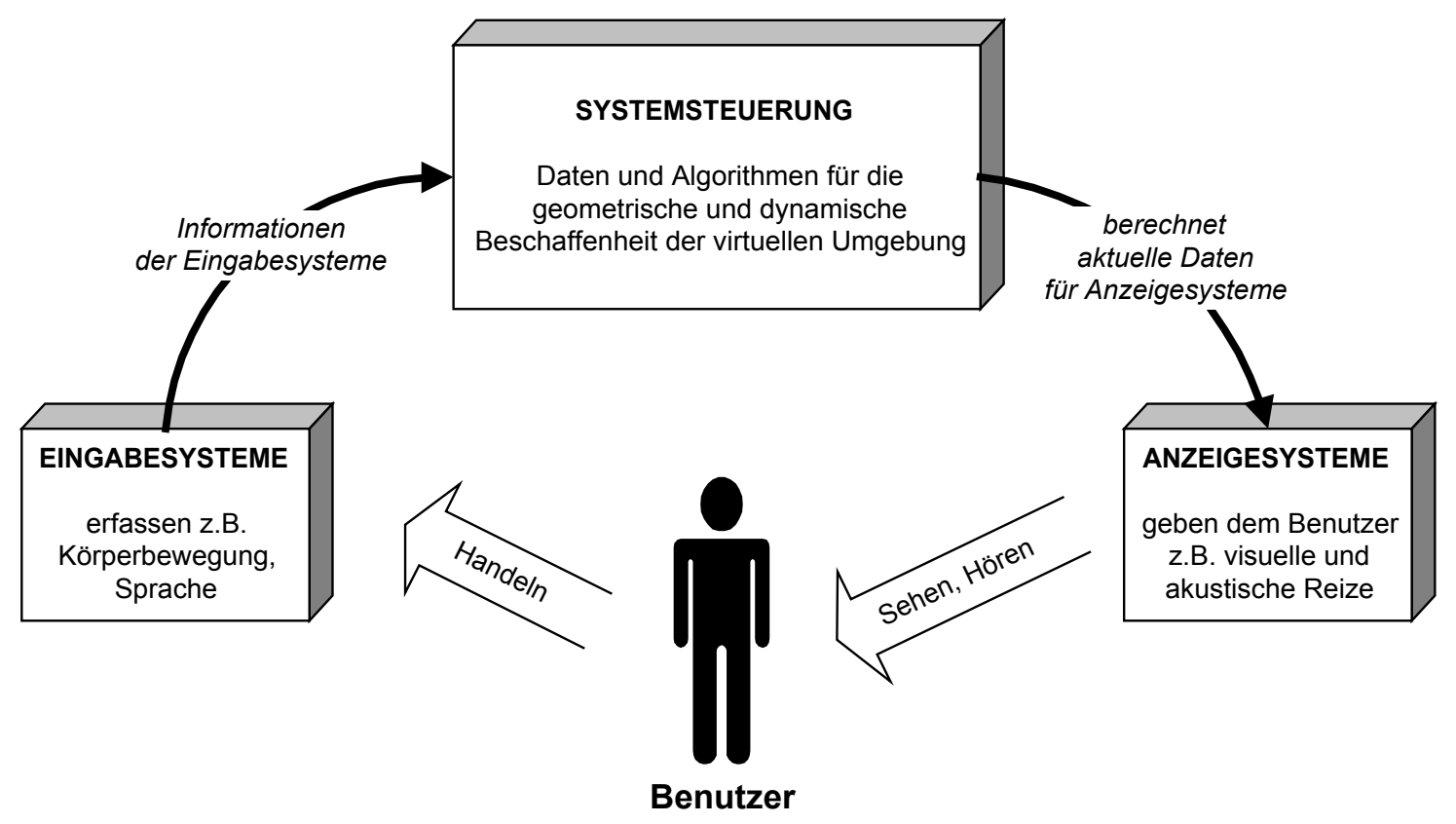

Abb 1.2. $\quad$ Schematische Darstellung der Systeme einer Virtual-Reality-Anwendung

\subsubsection{EINGABESYSTEME (REAL ENVIRONMENT SENSING)}

Unter Eingabesystemen versteht man Geräte, die der Benutzer zu Handlungszwecken (Interaktion) bedienen kann oder die Informationen aus der realen Umgebung messen können. Die vom Eingabesystem gewonnenen Daten können sehr unterschiedlicher Natur sein: Positionsdaten von Körperteilen, geometrische Informationen von Objekten, Sprache oder die 
Bewegung eines Steuerknüppels. In den meisten Fällen werden die Daten des Eingabegerätes zur Interaktion des Benutzers mit der virtuellen Umgebung verwendet.

Es existieren verschiedenste Systeme, um natürliche menschliche Interaktion abzubilden. Nach Youngblut et al. (1996) werden folgende Arten von Eingabe-Systemen unterschieden: Trackingsysteme, Taktil-Haptische Systeme, ,Primary-User-Input'-Systeme, ,Whole-Body Motion'-Systeme.

1. Tracking-Systeme (engl. to track: verfolgen) : Mit Sensoren werden Position (x, y, z) und Orientierung (Drehung um x-, y-, z-Achse) von Objekten im Raum erfasst. Beispiel: durch die Messung der Pupillenstellung wird die aktuelle Blickrichtung gewonnen.

2. Taktil-Haptische Systeme (taktil = die Berührung betreffend; haptisch $=$ den Tastsinn betreffend): In diesen Systemen sind Eingabe- und Ausgabeschnittstelle kombiniert: durch Sensoren werden Bewegungen von Körperteilen oder Werkzeugen gemessen. Stoßen diese in der virtuellen Umgebung auf ein Hindernis, erhält der Benutzer durch das System eine mechanische Rückmeldung (Kraftrückkopplung, engl. force feedback). Beispiel: ein an einem mechanischen Arm befestigtes Endoskop ermöglicht die Exploration eines Hohlorgans. Stößt man mit dem Endoskop gegen die Organwand, so vermittelt das System über den mechanischen Arm einen dynamischen Widerstand.

3. 'Primary-User-Input'-Systeme: dies sind Weiterentwicklungen von herkömmlichen Eingabegeräten (z.B. Maus) für die Verwendung bei räumlichen Aufgaben (z.B. 3D-Maus). Beispiel: Begehen eines Gebäudes bei einer Architektursimulation ohne Einschränkung der räumlichen Freiheitsgrade.

4. 'Whole-Body-Motion'-Systeme: Diese kombinierten Eingabe/Ausgabe-Systeme dienen einerseits dazu, Ganzkörperbewegungen zu erfassen, andererseits, dem Benutzer passive Bewegungen (Beschleunigung, Drehung) zu vermitteln. Beispiel: aufwändige VR-Spiele, hochrealistische Flugzeug-/ militärische Simulationen.

Neben den obengenannten Geräten werden für einfache Interaktionsaufgaben Standard-Mäuse oder Joysticks verwendet, obwohl diese nicht zur VR-Technologie gezählt werden. Für spezifische Aufgabensituationen, wie z.B. endoskopische Operationen, werden reale Instrumente mit taktil-haptischen Systemen bestückt, um die virtuelle und reale Interaktion möglichst ähnlich zu gestalten. Weiterhin können Spracheingabesysteme für verbale Befehle verwendet werden.

\subsubsection{ANZEIGESYSTEME (VIRTUAL ENVIRONMENT DISPLAY)}

Als Anzeigesysteme werden Geräte bezeichnet, die dem Benutzer sensible oder sensorische Informationen vermitteln. Dies sind auditorische, taktile, olfaktorische, thermische oder visuelle Reize zur Simulation einer Umgebung. 
Hauptaugenmerk bei vielen VR-Ausgabesystemen wird auf die visuelle Anzeige gelegt. Werden physiologische Charakteristika des Sehens als Maßstab für die Anzeige virtueller Umgebungen angelegt, so ergeben sich folgende Maximalansprüche (McKenna und Zeltzer 1992):

- die Anzeige sollte stereoskopes (räumliches) Sehen ermöglichen.

- die Darstellung sollte mit hoher graphischer Auflösung, Farbtreue und Helligkeit erfolgen.

- die Anzeige sollte das gesamte Gesichtsfeld einnehmen.

- Änderungen der Blickrichtung sollten ohne störende Verzögerung zur korrespondierenden Änderung des betrachteten Bildes führen.

Aus der praktischen Anwendung ergeben sich weitere Eigenschaften, die zu bewerten sind:

- die Benutzerfreundlichkeit des Systems und

- das Auftreten von Nebenwirkungen beim Anwender.

Eine Übersicht über wesentliche Anzeigesysteme wird in Tabelle 1.1. gegeben (Seite 8). Typische VR-Anzeigesysteme sind 'Head Mounted Displays' (HMD, sog. Datenbrillen; Sutherland 1968) und Mehr-Wand-Projektions-Systeme. Ihnen ist gemeinsam, dass sie eine stereoskopische, für den Betrachter 'greifbar' räumliche Darstellung bieten können. Das Konzept eines Anzeigesystems, welches das gesamte Blickfeld des Benutzers einnimmt, wird technisch unterschiedlich gelöst. Mehr. Wand-Projektions-Systeme (Abb. 1.3) sind begehbare Würfel mit bis zu sechs Projektionsflächen. Der Benutzer wird somit als reale Person Teil der virtuellen Umgebung. Beim Head Mounted Display (Abb. 1.4.) befinden sich zwei kleine Bildschirme wie Brillengläser vor den Augen. Das Blickfeld ist teilweise durch die Bildschirme eingenommen, der Rest wird durch die Abdeckungen des HMD verdeckt. Der eigene Körper kann daher vom Benutzer selbst nicht mehr visuell wahrgenomen werden.

In Zusammenhang mit Anzeigesystemen sind noch zwei Begriffe zu nennen, die häufig verwendet werden: als 'Desktop-VR' werden Systeme bezeichnet, bei denen ein

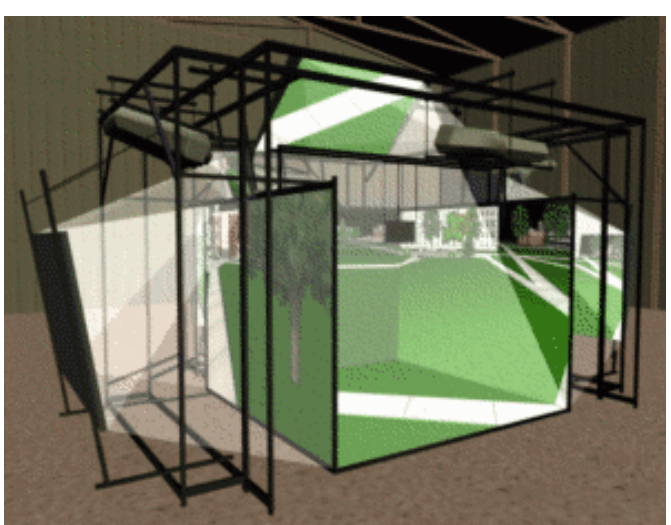

Abb. 1.3. Mehrwandprojektionssystem CAVE (Cruz-Neira et. al. 1993)

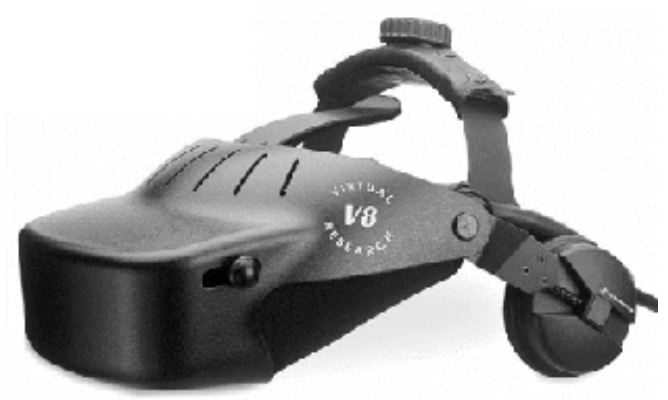

Abb. 1.4. Head Mounted Display (VirtualResearch Inc. 1998) 
stereoskopischer oder gewöhnlicher Bildschirm verwendet wird. Unter 'Augmented Reality' versteht man Systeme, die z.B. Datenbrillen mit transparenten Bildschirmen verwenden: so werden virtuelle Bildinformationen mit der realen Umgebung vermischt.

Akustische Stimuli sind für VR-Anwendungen lange vernachlässigt worden. Die VR-spezifische Präsentation von akustischen Stimuli zeichnet sich durch ihre Räumlichkeit aus. Dies bedeutet, dass der Benutzer z.B. einer Stimme oder einem Geräusch eine Position in der virtuellen Umgebung zuordnen kann. Weitere nicht-visuelle Anzeigesysteme sind KraftRückkopplungssysteme (engl. 'Force Feedback'), die bereits bei den taktilen Systemen im vorangehenden Abschnitt angeführt wurden.

Inwieweit visuelle Anzeigesysteme Einfluss auf die Immersion bzw. den Sense of Presence bei VR-Anwendungen haben, ist vielfach in Experimenten untersucht worden. Deisinger et al. (1997) ließen so z.B. VR-unerfahrene Benutzer einfache Navigationsaufgaben je mit Bildschirm, Head Mounted Display und einem Mehrwandprojektionssystem lösen und fragten nach Ihrer Präferenz und dem Immersionsgefühl. Vorgezogen wurde eindeutig das Projektionssystem, gefolgt vom Monitor. In die VR-Umgebung hineinversetzt fühlten sich die Probanden am ehesten mit dem Projektionssystem, ebenfalls mit dem HMD, kaum mit dem Monitor. Hendrix und Barfield (1996) beschreiben höhere Presence-Werte für Stereoskopie (vs. Monoskopie), bei Einsatz eines Kopf-Trackingsystems sowie einem Winkel des geometrischen Blickwinkels größer als $50^{\circ}$.

\subsubsection{STEUER- UND KONTROLLSYSTEME (VIRTUAL ENVIRONMENT CONTROL)}

\subsection{Hardware}

Die eingehenden Informationen der Eingabesysteme müssen verarbeitet und auszugebende Informationen für die Anzeigesysteme bereitgestellt werden. Dies geschieht mittels Graphikcomputer und VR-Software.

Bei den Graphikcomputern werden High-End-Rechner, Workstations und Personalcomputer unterschieden. High-End-Rechner, deren Anschaffungskosten bei bis zu mehreren Millionen Euro liegen, waren bis Anfang der neunziger Jahre die einzigen Systeme, die die aufwändigen Berechnungen zur Visualisierung und Datenverarbeitung durchführen konnten. Sie sind häufig Multiprozessorsysteme, deren interne Architektur auf die Verarbeitung sehr großer Datenmengen angelegt ist und die gleichzeitig von mehreren Benutzern verwendet werden können. Im Leistungsspektrum darunter befinden sich die sogenannten Workstations. Dies sind Einzelbenutzer-Rechner, deren interne Architektur auf graphische oder wissenschaftliche Aufgaben ausgerichtet ist. Personalcomputer sind Einzelbenutzer-Rechner, die aus Standardkomponenten gefertigt werden und für Büro-, Spiele- und einfache Graphikanwendungen benutzt werden. Rasante Weiterentwicklungen im Personalcomputer- 


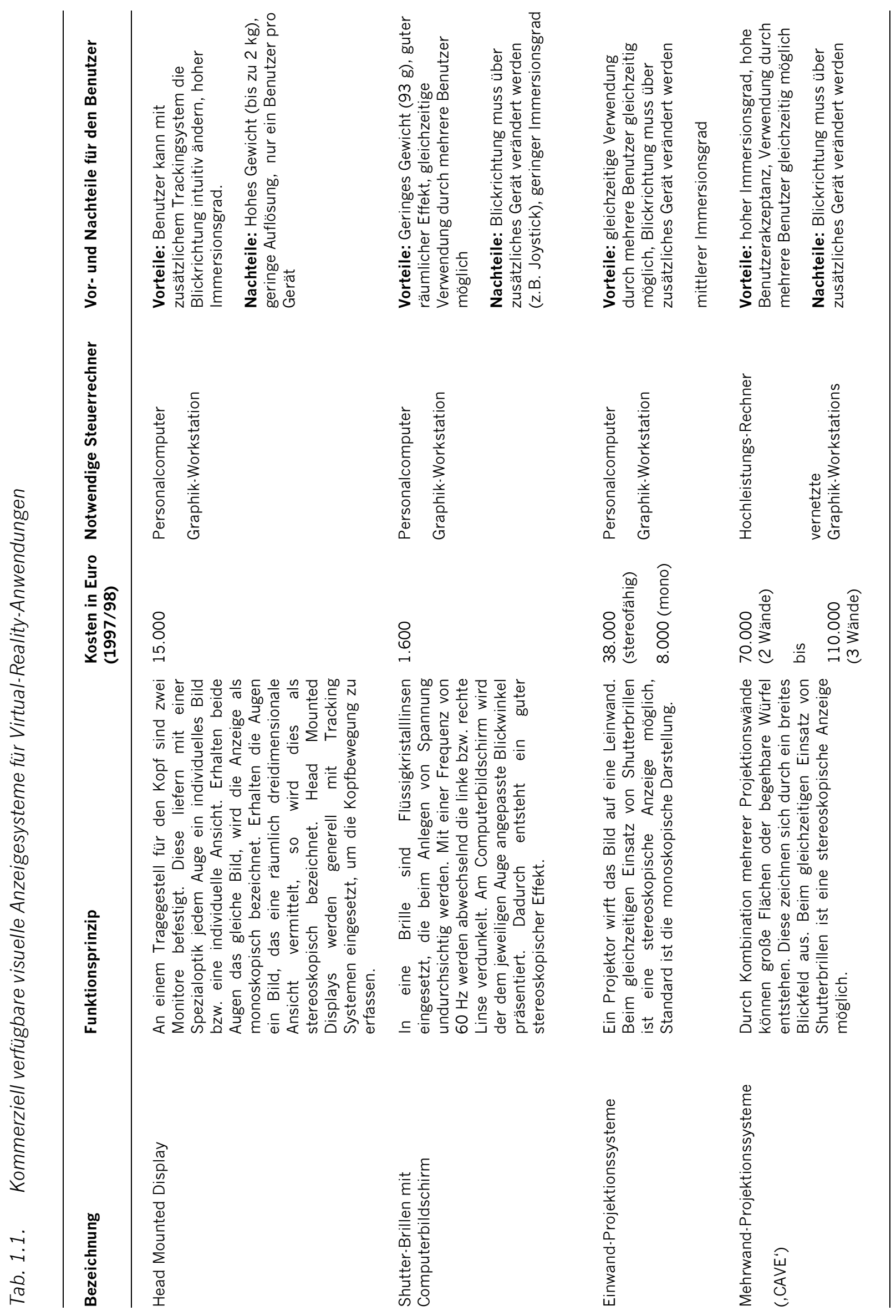


bereich erlauben inzwischen, die vormals High-End-Rechnern und Workstations vorbehaltenen Leistungsbereiche abzudecken, z.B. der Neuronavigation (Akatsuka et al. 2000). Diese Entwicklung ist vor allem durch die Spieleindustrie bedingt, die mit immer realistischeren Produkten hohe Anforderungen an die Rechner stellt. Neben der leistungsfähigeren Hardware im PC-Bereich (Prozessorgeschwindigkeit, Graphikkarten) revolutionieren vor allem Softwarelösungen zur Vernetzung und Synchronisation von PC-Graphiksystemen die Einsatzmöglichkeiten preisgünstigerer Systeme.

Eine Auswahl von Leistungsdaten einiger Graphikrechner bei Testmessungen zum Zeitpunkt der Projektplanungsphase geben Tabelle 1.2. und Abb.1.5.. Zu dem Testzeitpunkt existierte nur ein Gerät (SGI Onyx2), das bei komplexen VR-Umgebungen die Bildwiederholungsrate im physiologischen Bereich der visuellen Wahrnehmung produzieren konnte $(\sim 25 \mathrm{~Hz})$. Die Anschaffungskosten dieser High-End-Systeme betrugen das Vielfache der Workstations und von Personal-Computern. Abbildung 1.5. verdeutlicht noch einmal, dass für gute Bildwiederholungsraten und im besonderen für die Unix-basierten Systeme überdurchschnittliche Anschaffungskosten anfielen.

Tab.1.2. Leistungsdaten von ausgewählten Graphikcomputern zum Zeitpunkt der Projektplanungsphase (nach OpenGL Performance Characterization Group 1998)

\begin{tabular}{|c|c|c|c|c|c|}
\hline $\begin{array}{l}\text { Produkt- } \\
\text { bezeichnung }\end{array}$ & $\begin{array}{l}\text { Wichtige } \\
\text { Spezifikationen }\end{array}$ & $\begin{array}{l}\text { Betriebs- } \\
\text { system }\end{array}$ & $\begin{array}{l}\text { Preis in } \\
\text { US } \$ \\
(1998)\end{array}$ & $\begin{array}{l}\text { Durchschnittliche } \\
\text { Bildwieder- } \\
\text { holungsrate (BWR) }\end{array}$ & $\begin{array}{l}\text { Index } \\
\text { Preis } \\
\text { BWR }\end{array}$ \\
\hline $\begin{array}{l}\text { SGI Onyx2 DS } \\
\text { (High-End) }\end{array}$ & $\begin{array}{l}\text { Prozessor: } 2 \text { x } 195 \mathrm{MHz} \text { R10000 } \\
\text { Hauptspeicher: } 128 \mathrm{MB} \\
\text { Graphiksystem: InfiniteReality, } 80 \\
\text { MB Speicher, } 16 \mathrm{MB} \\
\text { Texturspeicher }\end{array}$ & Unix & 170120 & $45.43 / \mathrm{s}$ & 3744 \\
\hline $\begin{array}{l}\text { SGI Onyx2 } \\
\text { (High-End) }\end{array}$ & $\begin{array}{l}\text { Prozessor: } 180 \mathrm{MHz} \text { R10000 } \\
\text { Hauptspeicher: } 128 \mathrm{MB} \\
\text { Graphiksystem: Reality, } 40 \mathrm{MB} \\
\text { Speicher, } 16 \mathrm{MB} \text { Texturspeicher }\end{array}$ & Unix & 85120 & $23.70 / \mathrm{s}$ & 3592 \\
\hline $\begin{array}{l}\text { Hewlett Packard } \\
\text { Kayak XW } \\
\text { (Workstation) }\end{array}$ & $\begin{array}{l}\text { Prozessor: } 300 \mathrm{MHz} \text { Pentium II } \\
\text { Hauptspeicher: } 128 \mathrm{MB} \\
\text { Graphiksystem: HP Visualize fx4, } \\
\text { 34 MB Speicher, } 16 \mathrm{MB} \\
\text { Texturspeicher }\end{array}$ & Windows NT & 15907 & $18.04 / \mathrm{s}$ & 882 \\
\hline $\begin{array}{l}\text { SGI Octane } \\
\text { (Workstation) }\end{array}$ & $\begin{array}{l}\text { Prozessor: } 195 \mathrm{MHz} \text { R10000 } \\
\text { Hauptspeicher: } 128 \\
\text { Graphiksystem: MXI, } 27 \mathrm{MB} \\
\text { Speicher, } 4 \text { MB Texturspeicher }\end{array}$ & Unix & 45495 & $16.57 / \mathrm{s}$ & 2746 \\
\hline $\begin{array}{l}\text { Intergraph TDZ } \\
\text { (Workstation) }\end{array}$ & $\begin{array}{l}\text { Prozessor: } 300 \mathrm{MHz} \text { Pentium II } \\
\text { Hauptspeicher: } 128 \\
\text { Graphiksystem: RealiZM II, } 32 \text { MB } \\
\text { Speicher, } 4 \text { MB Texturspeicher }\end{array}$ & Windows NT & 14180 & $16.56 / \mathrm{s}$ & 856 \\
\hline $\begin{array}{l}\text { SNI Scenic } \\
\text { Celsius } 1000 \\
(P C)\end{array}$ & $\begin{array}{l}\text { Prozessor: } 300 \text { MHz Pentium II } \\
\text { Hauptspeicher: } 32 \\
\text { Graphiksystem: DiamondFire GL, } \\
15 \text { MB Speicher, } 16 \text { MB } \\
\text { Texturspeicher }\end{array}$ & Windows NT & 8470 & $9.98 / \mathrm{s}$ & 809 \\
\hline
\end{tabular}


Abb. 1.5. Preis in US-Dollar und Bildwiederholungsrate ausgewählter Graphikcomputer, Zeitpunkt Projektplanungsphase (OpenGL Performance Characterization Group 1998)

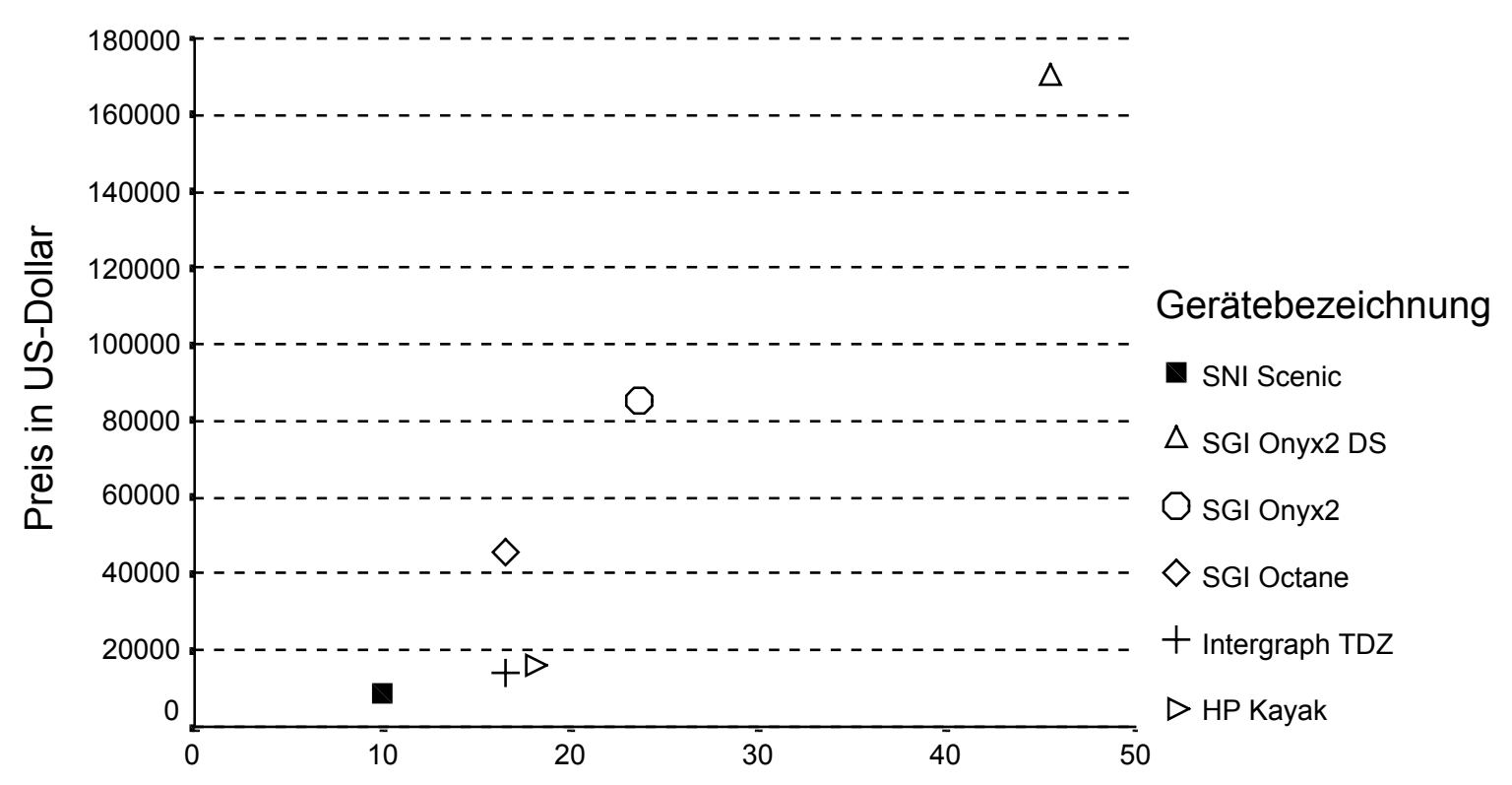

Bildwiederholungsrate pro Sekunde

Eine umfassende Übersicht über kommerzielle und als Prototypen vorhandene VR-Systeme ist von Youngblut erstellt worden (Youngblut et al. 1996).

\subsection{Software}

Bei der VR-Software müssen zwei Gruppen unterschieden werden: Entwicklungssoftware und Laufzeitsoftware.

Mit der Entwicklungssoftware werden Objekte und Funktionalität (physikalisches Verhalten, Aktionen) der Virtual-Reality-Umgebung erstellt. Dies geschieht zunächst mit Konstruktionssoftware, die die geometrischen Objekte und ihr Aussehen bestimmt. Mit VREntwicklungsprogrammen können diesen Objekten physikalische oder dynamische Eigenschaften verliehen werden, z.B. die Deformation einer Oberfläche bei Berührung oder das Ertönen eines Geräuschs beim Öffnen einer Tür.

Die Laufzeitsoftware liefert aus den Daten der Entwicklungssoftware die eigentliche virtuelle Umgebung. Sie stellt die geometrischen Objekte dar (z.B. eine Landschaft), registriert Handlungen des Benutzers (eine Kopfbewegung) über die Eingabesysteme und reagiert mit einer veränderten Darstellung über die Ausgabesysteme (Landschaft in neuer Blickrichtung).

Für viele chirurgische Anwendungen werden bei der Entwicklungssoftware zusätzlich Programme benötigt, die aus radiologischen Datensätzen (CT, MRT) durch mathematische Verfahren (Segmentierung) geometrische Objekte (Organdarstellungen) erstellen (Shin et al. 2000). 


\subsubsection{Unerwünschte Effekte bei der Benutzung virtueller Umgebungen}

\subsubsection{DEFINITION UND PATHOPHYSIOLOGIE}

Ähnlich den Kinetosen (eng/. Motion Sickness), wie sie zum Beispiel bei Autofahrten oder Achterbahnfahrten auftreten können, kann es bei der Verwendung von VR-Technologien beim Benutzer zum Auftreten von Symptomen wie Schwindel, Übelkeit bis hin zu Erbrechen, Kopfschmerz oder Müdigkeit der Augen kommen. Dieser Symptomkomplex, der vor allem aus der Verwendung von Flugzeugsimulatoren bekannt ist, wird im Kontext seiner Verwendung mit interaktiven Simulationen als 'Simulator Sickness' bezeichnet (Biocca 1992; Pausch et al. 1992).

Als Erklärung für das Auftreten der Simulator Sickness wird ein sogenanntes 'sensory-conflict'Modell herangezogen (Bles et al. 2000). Dabei wird angenommen, dass das Gehirn für intendierte Körperbewegungen bestimmte Erwartungen an das resultierende vestibuläre und sensorisch-sensible Feedback entwickelt. Unterscheidet sich das Feedback aufgrund einer veränderten sensorimotorischen Anordnung (engl. sensorimotor rearrangement), entsteht ein Konfliktsignal, das letztendlich für die Simulator-Sickness-Symptome verantwortlich ist (DiZio und Lackner 1992). VR-Anwendungen halten eine Vielzahl an veränderten sensorimotorischen Anordnungen bereit: simulierte aktive oder passive Bewegungen, Verzögerungen zwischen Interaktion und Antwort, geometrisch nicht korrekte Darstellungen oder die Beschränkung des sensorischen Feedbacks auf einzelne Modalitäten (DiZio und Lackner 1992).

\subsubsection{ERFASSUNG VON NEBENWIRKUNGEN}

Eine ausführliche Analyse des Simulator-Sickness-Symptomkomplexes haben Kennedy und Lane (1993) veröffentlicht, die bei 1119 Navy-Simulatoreinsätzen den für Kinetosen verwendeten Motion-Sickness-Questionnaire erhoben und anhand einer Faktorenanalyse die für Simulationen relevanten Symptome identifizierten. Resultierend aus der Faktorenanalyse wurde der 'Simulator Sickness Questionnaire' (SSQ) entwickelt, der inzwischen als etabliertes Instrument zur Erfassung von VR-Nebenwirkungen verwendet wird.

Nach Kennedy und Lane können drei voneinander unabhängige Symptomcluster unterschieden werden: der 'oculomotor'-Cluster mit verschwommenem Sehen, Schwierigkeiten zu fokussieren, ein 'nausea'-Cluster mit Übelkeit, erhöhtem Speichelfluss, Aufstoßen und ein 'disorientation'-Cluster mit Dreh- oder Schwankschwindel. Im SSQ werden zusätzlich allgemeines Unwohlsein, Konzentrationsschwierigkeiten und Schwitzen aufgeführt (s. SSQ u. Berechnung von SSQ Scores: Anhang 6.2./6.3.). Der aus Flugzeug.Simulatoren bekannte Effekt von Gleichgewichtsstörungen (z.B. Prothero et al. 1999) konnte nach VR-Anwendungen, vermutlich auch aufgrund methodischer Probleme, bisher nicht konsistent nachgewiesen werden (Kolasinski und Gilson 1999). 


\subsubsection{INDIVIDUELLE UND TECHNISCHE FAKTOREN MIT EINFLUSS AUF SIMULATOR- SICKNESS-SYMPTOME}

Die Inzidenz von Simulator-Sickness-Symptomen scheint sowohl von der individuellen Disposition, der technischen Realisation als auch von der Art der VR-Anwendung abhängig zu sein. Einen Überblick über Einflussfaktoren auf das Auftreten von Simulator-SicknessSymptomen gibt Kolasinski $(1995,1996)$. Wichtige, in empirischen Arbeiten nachgewiesene Einflussfaktoren sind in den Tabellen 1.3. und 1.4. angegeben.

Tab. 1.3. Auswahl individueller Faktoren, die mit einem verstärkten Auftreten von SimulatorSickness-Symptomen assoziiert sein können (nach Kolasinski 1995, 1996):

\begin{tabular}{ll}
\hline Faktor & Kommentar \\
\hline Alter & $\begin{array}{l}\text { Höchste Anfälligkeit zwischen } 2 \text { und } 12 \text { Jahren. Anfälligkeit fällt } \\
\text { danach sukzessive ab bis fast zum völligen Verschwinden um das } 50 . \\
\text { Lebensjahr. }\end{array}$ \\
$\begin{array}{l}\text { Erfahrung mit Simulatoren } \\
\text { Geschlecht }\end{array}$ & $\begin{array}{l}\text { Erfahrung mit VR senkt Anfälligkeit } \\
\text { Fähigkeit zur mentalen Rotation }\end{array}$ \\
\hline
\end{tabular}

Die Erkenntnisse zur Simulator Sickness und deren Auftreten unter verschiedenen technischen Realisationen und Anwendungen basieren zumeist auf Veröffentlichungen militärischer Simulationen, von Spielen oder Industrieapplikationen (Pausch et al. 1992, Kennedy et al. 1992, Cobb et al. 1999).

Publizierte Daten zum Auftreten von Nebenwirkungen unter verschiedenen VR. Anzeigekonfigurationen bei gleichem Anwendungsinhalt existieren lediglich im Vergleich von HMD und klassischem PC-Bildschirm. So brachen bei einem PC-Spiel 10\% der HMD-Benutzer das Experiment ab, keiner der Monitor-Benutzer. Nur bei der HMD-Gruppe kam es zur signifikanten Steigerung der SSQ-Scores (Naepflin und Menozzi 1998). Cobb et al. (1999) schildern in ihrer Arbeit, dass bei einem Experiment Nebenwirkungen mit HMD deutlich häufiger auftraten als mit einem klassischen Monitor.

Das Fehlen systematischer Untersuchungen zum Einfluss der Anzeigemodalität auf die Nebenwirkungen spiegelt sich in der Inhomogenität publizierter Daten wider. Bei Dokumentation mittels SSQ werden z.B. mit HMD ein Anstieg der SSQ Scores bei $61.80 \%$ der Probanden mit einer Abbrecherquote von 5\% beschrieben (Regan und Price 1994, Cobb et al. 1999), während andere Arbeitsgruppen 'keine' Nebenwirkungen bei Verwendung eines HMD beschreiben (Christiansen et al. 1998, Riva 1998). Für das Mehrwandprojektionssystem 'CAVE' sind Nebenwirkungen bei 2 von etwa 9000 Besuchern (Cruz-Neira et al. 1993) beschrieben. Stanney und Hash (1998) beschreiben bei einer Navigationsaufgabe mit stereoskopischem Bildschirm bei 95\% der Probanden einen Anstieg der mittels SSQ erfassten Symptome. 
Tab. 1.4. Auswahl technischer Faktoren, die mit einem verstärkten Auftreten von SimulatorSickness-Symptomen assoziiert sein können (nach Kolasinski 1995, 1996):

\begin{tabular}{ll}
\hline Faktor & Kommentar \\
\hline Binokulares Sehen & Stereoskopie führt zu stärkerer Anfälligkeit \\
Field of View (FOV = Breite des Gesichtsfeldes) & Höherer FOV führt zu stärkerer Anfälligkeit \\
Einsatz eines Tracking Systems & $\begin{array}{l}\text { Geometrische Verzerrungen und andere Fehlerquellen können zu } \\
\text { stärkerer Anfälligketi führen }\end{array}$ \\
Bildwiederholungsrate, Interaktionspause & $\begin{array}{l}\text { Jede Verzögerung in der Anzeige, z.B. durch geringe } \\
\text { Bildwiederholungsrate oder lange Interaktionspause, kann zu } \\
\text { stärkerer Anfälligkeit führen } \\
\text { Geschwindigkeit }\end{array}$ \\
\hline
\end{tabular}

Es existieren nur wenige Publikationen, die sich mit dem Auftreten von Simulator-SicknessSymptomen bei medizinischen Anwendungen beschäftigen. Eine systematische Erfassung und Auswertung von Nebenwirkungen fand bisher nicht statt. Bei Patienten mit diagnostizierter Alzheimer-Erkrankung wurden in einem Autosimulator die Fahrleistungen überprüft, der technische Aufbau entsprach mit drei 25-Zoll-Bildschirmen einem Blickwinkel von $165^{\circ} .29$ Probanden starteten den Versuch, 10 brachen wegen Konfusion, zwei aufgrund SimulatorSickness-Symptomen ab (Cox et al. 1998). Christiansen beschreibt in einer Anwendung zur Rehabilitation von Patienten nach Schädel-Hirn-Trauma mittels HMD keinerlei Nebenwirkungen (Christiansen et al. 1998), ebenso Skelton et al. (2000) bei einer Navigationsaufgabe mit Monitor. Bei einem VR-Programm zur Modifikation des Körperselbstbildes mittels HMD. Konfiguration wurden in einer vorklinischen Studie bei 2 von 71 Probanden SimulatorSickness-Symptome beschrieben (Riva 1997), in einer folgenden klinischen Studie mit 24 Probanden und 24 Patienten 'keine' Nebenwirkungen (Riva 1998). In einer Studie zum topographischen Lernen mit 34 Patienten nach unilateraler temporaler Lobektomie und 21 Kontrollprobanden mittels Monitorkonfiguration brachen wegen Übelkeit 2 der Patienten und 3 der Probanden ab, was einer Quote von 9\% entspricht (Spiers et al. 2001).

\subsubsection{BEHANDLUNG DER NEBENWIRKUNGEN}

Eine medikamentöse Behandlung bei Auftreten von Simulator-Sickness-Symptomen ist in der Regel nicht notwendig, nach Entfernung des Stimulus sind die Symptome meist rasch rückläufig und nach wenigen Stunden vollständig verschwunden (z.B. Cobb et al. 1999). Falls eine Behandlung notwendig wird, kann diese - angelehnt an die Kinetosenbehandlung - mit Antiemetika (Antidopaminergika, z.B. Metoclopramid; Anticholinergika, z.B. Scopolamin; Antihistaminika, z.B. Diphenhydramin) durchgeführt werden (Gahlinger 1999). Im Rahmen einer placebokontrollierten Studie zeigte sich bei Militärsimulationen, dass eine Einnahme von Hyoscinbutylbromid (antimuskarinisches Parasympatholytikum, z.B. Buscopan®) 40 Minuten vor der VR-Anwendung signifikant zur Minderung von Symptomen beitrug (Regan 1995). 


\subsubsection{PROBLEME UND OFFENE FRAGEN HINSICHTLICH UNERWÜNSCHTER NEBENWIRKUNGEN}

Das Auftreten unerwünschter Effekte wird als schwerwiegendes Hindernis für eine breite Anwendung von VR-Technologien gesehen (Biocca 1992) und hat im Hinblick auf medizinische Einsatzgebiete ethische Bedenken (Risiko vs. Nutzen) hervorgerufen (s.a. Kap. 1.2.4.). Die Datenlage zum Auftreten von Nebenwirkungen bei VR-Anwendungen ist - vor allem im medizinischen Bereich - unzureichend für eine Bewertung bezüglich der Anwendersicherheit (Rizzo et al. 1998a). Im Rahmen eines Symposiums wurden daher von führenden Wissenschaftlern zukünftige Forschungsschwerpunkte formuliert (Stanney et al. 1998), u.a. 'Pathophysiologie der Simulator Sickness', 'prädiktive Modelle zur Bestimmung der Anfälligkeit von Personen' und 'Etablierung von Richtlinien zum Design von VR-Anwendungen'.

\subsubsection{Medizinische Anwendung von VR}

\subsubsection{1. ÜBERBLICK UND BEISPIELE VON EINSATZGEBIETEN}

Bullinger \& Bauer (1994) sehen neben den Ingenieurwissenschaften und der Architektur in der Medizin den wissenschaftlichen Bereich, der das größte Potential von anwendungsorientierten VR-Entwicklungen aufweist. Die Einsatzgebiete reichen von der medizinischen Aus- und Fortbildung (Berlage 1997) über den therapeutischen Einsatz im Rahmen der Operationsunterstützung (Grönemeyer et al. 1996) bis hin zu anwendungsorientierten Studien auf dem Gebiet der humanen Neurowissenschaften (Glantz et al. 1997). Als qualitativ neue Einsatzmöglichkeiten wird z.B. der Ersatz von Coloskopien im Rahmen von Vorsorgeuntersuchungen durch virtuelle Endoskopien mit aus Computertomographien oder Kernspintomographien gewonnenen, 3D-rekonstruierten Daten gesehen (Fenlon et al. 1999, Székely und Satava 1999). In den Neurowissenschaften bietet Virtual Reality die Möglichkeit, Umgebungen mit beliebigen Eigenschaften zu kreieren und dabei motorische oder kognitive Prozesse zu untersuchen (Carrozzo und Lacquaniti 1998).

Die Anwendung von VR in der Medizin kann anhand des primären, d.h. des mit der virtuellen Umgebung interagierenden Benutzers klassifiziert werden (Tab. 1.5.):

- dem Patienten oder

- ärztlichem, medizinischem oder medizinisch-technischem Personal.

Patienten-basierte VR beruht zumeist auf dem Prinzip der Interaktion des Patienten mit der virtuellen Umgebung. Die Handlung des Patienten besitzt einen therapeutischen bzw. rehabilitativen Effekt oder erlaubt eine diagnostische Aussage. In erster Linie sind dies neurologische oder psychiatrische Anwendungen (Glantz et al. 1997). Dem gegenüber stehen VR-Realisationen, anhand derer medizinisches Personal mit Patientendaten anatomische 
Strukturen interaktiv simulieren kann, so z.B. im Rahmen von virtuellen Endoskopien in den Einsatzgebieten Ausbildung, Diagnostik und Therapie (z.B. Müller et al. 1995).

Tab. 1.5. Typische Einsatzfelder und Anwendungsarten von VR-Technologien in der Medizin mit exemplarischen Anwendungen (modifiziert nach Mehlitz et. al. 1998).

\begin{tabular}{|c|c|c|}
\hline Einsatzfeld & $\begin{array}{l}\text { Anwendungsart } \\
\text { VR mit Patientendaten oder Modellen } \\
\text { - Arzt interagiert mit Computer - }\end{array}$ & $\begin{array}{l}\text { VR mit künstlichen Umgebungen } \\
\text { - Patient interagiert mit Computer - }\end{array}$ \\
\hline Diagnostik & $\begin{array}{l}\text { Dreidimensionale, interaktive Aufbereitung } \\
\text { radiologischer Daten zur nicht-invasiven } \\
\text { Diagnostik, z.B.: } \\
\text { virtuelle Koloskopie (Fenlon et al. 1999) }\end{array}$ & $\begin{array}{l}\text { Aufgabenstellungen mit Operationalisierung von } \\
\text { motorischen oder kognitiven Funktionen, z.B.: } \\
\text { neurophysiologische Testverfahren zur Diagnostik } \\
\text { von Bewegungsstörungen (Kuhlen et al. 1996) }\end{array}$ \\
\hline Therapie & $\begin{array}{l}\text { Präoperative Planung und intraoperative } \\
\text { Navigation mittels 3D-Visualisierung, z.B.: } \\
\text { Neuronavigation (Haberland et al. 1997) }\end{array}$ & $\begin{array}{l}\text { Situationen und Szenarien mit therapeutischem } \\
\text { Effekt, z.B.: } \\
\text { Systematische Desensibilisierung von Phobien } \\
\text { durch virtuelle 'in-vitro'-Exposition, z.B. bei } \\
\text { Höhenangst (Rothbaum et al. 1995) }\end{array}$ \\
\hline Ausbildung & $\begin{array}{l}\text { Simulation invasiver oder lern-intensiver } \\
\text { Verfahren, z.B.: } \\
\text { virtuelle Arthroskopie (Müller et al. 1995), } \\
\text { virtuelle Echokardiographie (Berlage 1997) }\end{array}$ & \\
\hline Rehabilitation & & $\begin{array}{l}\text { Rehabilitation von motorischen und kognitiven } \\
\text { Beeinträchtigungen, z.B.: } \\
\text { Schlaganfall-Patienten (Johnson et al. 1998) }\end{array}$ \\
\hline $\begin{array}{l}\text { Grundlagen- } \\
\text { forschung }\end{array}$ & & $\begin{array}{l}\text { Neurowissenschaftliche Forschung, z.B.: } \\
\text { Identifizierung beteiligter anatomischer Strukturen } \\
\text { bei kognitiven Prozessen (Maguire et al. 1998) }\end{array}$ \\
\hline
\end{tabular}

Für medizinische Anwendungen der VR bestehen je nach Anwendungsart unterschiedliche Anforderungen an die technische Realisation: in den chirurgischen Fächern werden sehr hohe technische Anforderungen, z.B. an das Objektverhalten (Blutgefäße, Gewebe) und die taktilhaptische Interaktionsfähigkeit in Echtzeit gestellt (Shin et al. 2000, Székely et al. 1998). Diese werden oft nur unzureichend erfüllt (Durlach und Mavor 1995). Im Gegensatz hierzu existieren aufgrund des Anwendungscharakters in der Neuropsychologie und Psychiatrie einfache und effektive VR-Realisationen (Johnson et al. 1998).

Der Einsatz von VR in der Medizin ist aus ethischer Sicht bereits früh kritisch betrachtet worden (Anonymous 1991). Dies ist einerseits in den geringen Erkenntnissen begründet, die über körperliche Nebenwirkungen bei klinischen VR-Anwendungen existieren; empirische Daten zum Auftreten der Simulator Sickness, z.B. bei der Benutzung durch neurologische oder psychiatrische Patienten, liegen bisher nur unsystematisch und nicht standardisiert vor. Darüberhinaus wird in Frage gestellt, ob eine Verwendung von künstlichen Umgebungen bei psychiatrisch erkrankten Personen, die z.B. über eine gestörte Realitätswahrnehmung oder eine Suchtsymptomatik verfügen, vertretbar ist (Whalley 1995). 


\subsubsection{VR ALS WERKZEUG IN DEN NEUROWISSENSCHAFTEN}

Wesentliche Gründe für die Verwendung von VR-Technologien für neurowissenschaftliche Fragestellungen sind die präzise Kontrollfähigkeit der Teststimuli und die technisch einfache Erfassbarkeit von Verhaltensdaten in realistischen Situationen oder Aufgaben (Gillner und Mallot 1998, Carrozzo und Lacquaniti 1998). In der Psychiatrie wird VR vor allem für verhaltenstherapeutische Ansätze genutzt, so bei Angsterkrankungen (Rothbaum et al. 1995, Botella et al. 1998) oder Störungen des Körperselbstbildes (Riva 1998). In der neurologischen Rehabilitation besitzt VR Anwendungsfelder nach Schädel-Hirn-Trauma bei Kindern (Rose et al. 1997) und Erwachsenen (Johnson et al. 1998, Christiansen et al. 1998) oder bei motorisch beeinträchtigten Patienten (Kuhlen 1996). In der Neuropsychologie sind Untersuchungen zu exekutiven Funktionen (Pugnetti 1995b) und zur topographischen Orientierung (Gillner und Mallot 1998) mittels VR durchgeführt worden, in neueren Studien vor allem im Einsatz mit funktionellen Bildgebungsverfahren (fMRI: Grön et al. 2000, PET: Maguire et al. 1998).

\subsection{Neuropsychologische Grundlagen}

\subsubsection{Anwendungsgebiete neuropsychologischer Testverfahren}

Neuropsychologische Testverfahren werden verwendet, um Hirnleistungen von Patienten mit neurologischen oder psychiatrischen Erkrankungen zu überprüfen. Übergeordnete Kategorien der funktionellen Systeme des Gehirns sind Sprache, Lernen/Gedächtnis, Wahrnehmung, Intelligenz und Aufmerksamkeit (Lezak 1995). Mit der neuropsychologischen Testung werden verschiedene Ziele verfolgt: Unterstützung der Diagnostik zur medizinischen oder psychologischen Intervention, Krankheitsmanagement, Prognosenabschätzung, Kontrolle des Krankheitsverlaufs und wissenschaftliche Fragestellungen (Beaumont 1996, Lezak 1995).

\subsubsection{Neuropsychologische Funktionen mit Relevanz für das Testsystem}

Das Virtual-Reality-gestützte Testsystem wurde für den Einsatz in der neuropsychologischen Diagnostik der exekutiven Funktionen, des visuell-räumlichen Lernens und der topographischen Orientierung vorgesehen.

Als exekutive Funktionen des Gehirns werden in der Neuropsychologie im wesentlichen die Fähigkeit der Konzeptbildung und des Konzeptwechsels, der Kategorisierung, der Verhaltensplanung, der Aufmerksamkeit und des Affekts genannt (Stuss und Benson 1986, Stuss und Alexander 2000). Neuroanatomisch werden die exekutiven Funktionen in erster Linie dem präfrontalen Kortex zugeordnet, wobei dieser wahrscheinlich nur ein übergeordnetes Zentrum eines ausgedehnten Netzwerkes kortikaler und subkortikaler Strukturen ist (Smith und Jonides 1999, Mattes-Cramon und Cramon 2000). Gegenwärtig existieren keine neuropsychologischen Testverfahren, die exekutive Funktionen adäquat operationalisieren 
(Stuss und Benson 1986, Lezak 1995, Mattes-Cramon und Cramon 2000). Dies hat zur Folge, dass z.T. Patienten mit großen frontalen Defekten und deutlichen Problemen im Alltagsleben in neuropsychologischen Tests zu exekutiven Funktionen Normalleistungen erreichen (Tranel 1994).

Visuell-räumliche Leistungen sind notwendig, um Objekte, deren Größe und Form, Konfiguration, Orientierung und Position zu erkennen (Kerkhoff 2000). Neuroanatomischfunktionell werden ausgehend vom occipitalen visuellen Cortex zwei getrennte cortikale Systeme beschrieben (Ungerleider et al. 1998, Poucet 1993): objektbezogene Informationen (Form, Farbe) gehen von dort aus in den inferioren Temporalcortex (sog. ventraler Pfad, ,what'). Räumliche Informationen gelangen vom visuellen Cortex in den posterioren Parietalcortex (sog. dorsaler Pfad, ,where'). Im inferioren Temporalcortex erfolgt die Objekterkennung und deren mentale Repräsentation. Aguirre vermutet aufgrund der Ergebnisse bildgebender, neuropsychologischer und tierexperimenteller Arbeiten, das der Gyrus parahippokampalis die Informationen des ventralen und dorsalen Pfades assoziiert (Aguirre et al. 1998).

Topographisch-räumliches Wissen ist notwendig, um sich bei der Fortbewegung in der Umwelt orientieren zu können. Im Hinblick auf visuell-räumliches Lernen handelt es sich bei der topographischen Orientierung um eine Leistung des Gehirns, bei der nicht nur räumliche Konfigurationen erlernt werden, sondern eine Kenntnis der Anordnung und Abfolge von Wegen und Objekten anhand von Landmarken (topographischen Merkmalen) erworben wird (Aguirre und D'Esposito 1999). Dem medialen Temporallappen, insbesondere den hippokampalen und parahippokampalen Strukturen, wird eine entscheidende Rolle beim Neuerwerb topographischen Wissens beigemessen (Aguirre et al. 1998).

Bei der Untersuchung räumlicher Gedächtnisleistungen lassen sich weiterhin zwei mentale Repräsentationsarten räumlichen Wissens unterscheiden: die allozentrische sowie die egozentrische Repräsentation. Bei der allozentrischen Repräsentation einer räumlichen Konfiguration wird diese unter Zuhilfenahme von Objekten (engl. landmarks) gespeichert. Dies entspricht z.B. dem Lernen eines Weges in einer Landschaft und wird im engeren Sinne als „topographisches Lernen“ bezeichnet. Wenn eine räumliche Konfiguration zu erlernen ist, bei der keine zusätzlichen äußeren Anhaltspunkte zur Verfügung stehen (z.B. ein Haus mit leeren Räumen), so müssen in starkem Maße propriozeptive Signale (Kopfhaltung, Blickrichtung, Körperposition, Bewegung) dazu verwendet werden, eine mentales Bild der Umgebung zu schaffen. Dieses wird als egozentrische Repräsentation von räumlichem Wissen bezeichnet.

In funktionell-bildgebenden Untersuchungen konnte gezeigt werden, dass allozentrisches und egozentrisches Lernen nicht allein theoretische Konstrukte sind, sondern dass diesen unterschiedliche neuronale Aktivierungen zugrunde liegen. Bei einer Lernaufgabe in einer virtuellen Umgebung mit und ohne Landmarken zeigte sich in fMRI-Studien, dass mit 
Landmarken eine deutliche medio-temporale Aktivierung im Bereich des Gyrus parahippokampalis bestand, während ohne Landmarken hier keine Aktivierung bestand (Maguire et al. 1998). Hartley et al. (2003) berichten von parahippokampaler Aktivierung im fMRI beim Neuerwerb allozentrischen Wissens, während beim reinen Abgehen eines festgelegten Weges eine Aktivierung des medialen Temporallappens quasi fehlt. Einzelfallberichte von Patienten mit einer bilateralen hippokampalen Pathologie zeigten deutliche Beeinträchtigungen des allozentrischen Lernens, während die egozentrischen Testleistungen nur wenig eingeschränkt waren (Holdstock et al. 2000, King et al. 2002). In Serien mit Patienten mit medikamentenresistenter Epilepsie nach unilateraler Temporallappenchirurgie zeigte sich sowohl bei links- wie rechtsseitigen Eingriffen eine deutliche Beeinträchtigung allozentrischer Lernleistungen gegenüber den Kontrollprobanden (Maguire et al. 1996, Astur et al. 2002). Hinsichtlich der Lateralität bestehen keine einheitlichen Ergebnisse (s. Maguire et al. 1998), jedoch wird räumlich-topographisches Lernen eher dem rechten medialen Temporallappen zugeordnet (Burgess 2002).
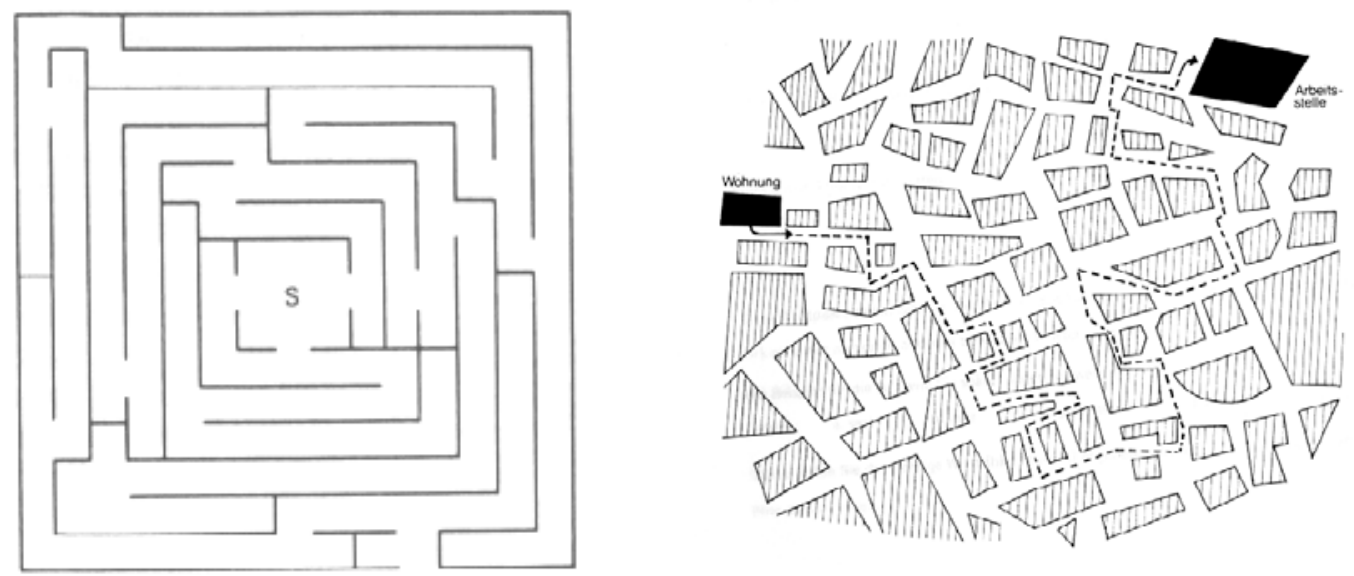

Abb. 1.6. u. 1.7.

Beispiele für konventionelle Paper \& Pencil-Tests zu visuellem Lernen und topographischer Orientierung: links Porteus Maze (nach Porteus 1959) und rechts LGT-3: Stadtplan, Lernvorlage (Bäumler 1974)

Bestehende verbreitete Testverfahren zu topographischem Lernen werden als unzureichend erachtet (Lezak 1995). Als besonders kritisch wird die sog. ,ökologische Validität' der Paper \& Pencil-Verfahren gesehen (Kindsmüller et al. 1999), wonach Testleistung und Alltagsleistung nur schlecht korrelieren (Aguirre und D'Esposito 1999). Den Standardtests fehlt z.B. als wichtiges Merkmal der Perspektivenwechsel (Maguire et al. 1996). Die Abbildungen 1.6. und 1.7. zeigen zwei Beispiele von Paper \& Pencil-Tests, den Porteus Maze sowie den LGT-3 Stadtplan. Es wird deutlich, dass zur Bearbeitung dieser zweidimensionalen Aufgaben nicht dieselben kognitiven Fähigkeiten notwendig sind wie zur räumlichen Orientierung im Alltag. 


\subsection{3. Überblick über Virtual-Reality-gestützte Anwendungen im Bereich der Neuropsychologie}

Virtual Reality wird als eine mögliche Methode gesehen, eine höhere ökologische Validität für Testverfahren bei standardisierten Versuchsbedingungen zu erreichen (Pugnetti et al. 1995a, Rizzo et al. 1998b). VR-Anwendungen wurden bereits für visuell-räumliches Lernen zur Untersuchung kognitiver Prozesse bei gesunden Testpersonen eingesetzt (z.B. Gillner und Mallot 1998, Astur et al. 1998), bei Personen nach Schädel-Hirn-Trauma (Skelton et al. 2000) und bei geistig-körperlich behinderten Kindern (Stanton et al. 1998). Zur Identifizierung der beteiligten neuroanatomischen Strukturen und Regionen wurden während VR-Aufgaben neurophysiologische und funktionell-neuroradiologische Verfahren wie EEG (Pugnetti et al. 1995a), subdurales EEG (Kahana et al. 1999), PET (Maguire et al. 1998) oder fMRI eingesetzt (z.B. Grön et al. 2000).

\subsection{Ziele und Fragestellungen der Arbeit}

Das Ziel der vorliegenden Arbeit war es, den Zugang zu einer neuartigen Technologie ('Virtual Reality', VR) am Klinikum der Universität Göttingen zu schaffen, eine medizinisch-technische Anwendung (neuropsychologisches Testsystem) auf Grundlage dieser Technik zu entwickeln und diese einer klinisch-technischen Prüfung zu unterziehen.

Es wurden dahingehend drei übergeordnete Ziele definiert, innerhalb derer verschiedene Fragestellungen bearbeitet werden sollten:

(A) Planung und Aufbau eines interdisziplinär nutzbaren, medizinischen Virtual-Reality-Labors.

(B) Technische Entwicklung eines VR-gestützten neuropsychologischen Testsystems.

(C) Präklinische und klinische Überprüfung des Testsystems mittels einer Evaluationsstudie hinsichtlich bekannter VR-bedingter Wirkungen und Nebenwirkungen sowie der Praktikabilität.

Die vorliegende Arbeit enthält sowohl theoretische als auch angewandte medizininformatische Teile. Die Laborplanung und die Vorüberlegungen zur Testentwicklung basieren auf theoretischen Vorarbeiten, während die Realisation und Evaluation des Testsystems vor allem angewandte medizininformatische Aspekte betrifft. Die mit der Testentwicklung verbundenen umfangreichen neuropsychologischen Fragestellungen (Operationalisierung, Validität und Reliabilität des Tests) sind nicht Bestandteil dieser Arbeit. Allerdings sollen im Rahmen von Punkt (C) auch empirische Daten zur Risikobewertung der VR-Technologie für ethische Fragestellungen gewonnen werden. 


\subsubsection{Planung und Aufbau eines interdisziplinären, medizinischen VR-Labors}

Bei der Planung des Virtual-Reality-Labors stellten sich folgende Fragen:

- Wie sieht die notwendige technische Grundausstattung für die Entwicklung von medizinischen VR-Anwendungen aus?

- Welche Basiskomponenten müssen vorhanden sein, damit z.B. chirurgische wie psychiatrische VR-Anwendungen realisiert werden können?

Anhand dieser Fragestellungen wurden folgende Ziele definiert:

(A.1) Identifizierung der verwendeten Technik existierender medizinischer VR-Projekte anhand einer Literaturanalyse.

Zu Beginn der Laborplanung war vorgesehen, neben informellen Quellen (z.B. Besuche von Kompetenzzentren im Bereich VR) bereits publizierte medizinische VR.Anwendungen bezüglich ihrer technischen Ausstattung und Anwendungsart zu analysieren, um repräsentative Daten als Entscheidungsgrundlage für die spätere Laboreinrichtung zu erhalten.

(A.2) Einrichtung eines Labors mit technischen Komponenten zur Entwicklung medizinischer VirtualReality-gestützter Anwendungen.

Anhand der durch die Literaturanalyse gewonnenen Informationen sollte unter Berücksichtigung von Kosten, bekannten Nebenwirkungen und technischer Realisierbarkeit ein VR-Labor eingerichtet werden. Das VR-Labor sollte eine ausreichende Geräte- und SoftwareAusstattung besitzen, um Projekte bis zum Prototypstadium realisieren zu können.

(A.3) Entwicklung eines organisatorischen Konzepts für den Betrieb eines interdisziplinär genutzten Virtual-Reality-Labors.

Durch die Bereitstellung von technischen und personellen Mitteln im VR-Labor sollte klinischen Abteilungen die Möglichkeit gegeben werden, VR-Anwendungen ohne eigene vertiefte technische Kenntnisse entwickeln zu können. Für die Projektarbeit im VR-Labor wurde der Entwurf eines organisatorischen Rahmens für notwendig erachtet.

\subsubsection{Entwicklung eines neuropsychologischen Testsystems}

Erstes Projekt innerhalb des VR-Labors war die Entwicklung eines neuropsychologischen Testsystems. Inhaltlich wurden Aufgabenoperationalisierungen zu visuell-räumlichem, räumlich-topographischem und exekutivem Lernen geplant. Bei der technischen Entwicklung wurden neben besonderer Berücksichtigung der neuropsychologischen Grundlagen folgende Ziele definiert: 
(B.1) Zielgruppenorientierte Entwicklung der Benutzerschnittstelle und der Aufgabensituation.

Trotz des Potentials von VR als Werkzeug in der Neuropsychologie wird deren Anwendung als kritisch betrachtet, auch im Hinblick auf die Benutzbarkeit durch die intendierte Zielgruppe (Patienten mit neurologischen Defiziten, höheres Lebensalter, geringe Computervorkenntnisse). Daher wurde für die Realisation des Testsystems eine möglichst einfaches, jedoch hinreichende Benutzerinteraktion angestrebt. Hiermit sollte gewährleistet werden, dass die Ergebnisse der neuropsychologischen Aufgabenbearbeitung nicht die Fähigkeit zur Computernutzung, sondern den Lernerfolg bei den neuropsychologischen Aufgaben zeigen.

(B.2) Automatisierte Erfassung, Reproduktion und Auswertung der Testleistung.

Hierbei sollten die Vorteile der Verwendung von Virtual Reality für neurowissenschaftliche Fragestellungen gegenüber Paper\&Pencil-Verfahren genutzt werden: die präzise Kontrollfähigkeit der Teststimuli und die technisch einfache Erfassbarkeit von Verhaltensdaten in realistischen Situationen oder Aufgaben.

(B.3) Verbesserung der ökologischen Validität durch VR.

Die ökologische Validität - d.h. im wesentlichen die Korrelation von Alltags- und Testleistung ist ein grundlegender Kritikpunkt an Paper \& Pencil-Tests. Zur Verbesserung dieses Aspektes sollten die technischen Möglichkeiten der virtuellen Realität beitragen - z.B. durch visuellen Realismus und realistische Interaktion bei der Aufgabenbearbeitung.

(B.4) Minimierung von Faktoren, die zum Auftreten von Simulator-Sickness-Symptomen führen konnten.

Bei der Entwicklung sollten bekannte Faktoren zur Entstehung der Simulator Sickness berücksichtigt werden, um eine größtmögliche Sicherheit für die Probanden zu erreichen. Die technische Entwicklung des Testsystems sollte im Rahmen eines methodisch präzisierten Vorgehens erfolgen, wie dies für die Softwareentwicklung gefordert und empfohlen wird.

\subsubsection{Evaluationsstudien zur Anwendung des Testsystems}

Eine Evaluationsstudie sollte als integraler Bestandteil der Systementwicklung Aufschlüsse hinsichtlich folgender Aspekte des neuentwickelten VR-gestützten Testsystems erbringen:

1. Praktikabilität und Benutzerfreundlichkeit des Testsystems

2. Wirkungen virtueller Realität

3. Nebenwirkungen virtueller Realität

Hierbei wurde zeitlich und methodisch ein zweistufiges Vorgehen vorgesehen:

- Zunächst sollte nach Testentwicklung eine präklinische Evaluationsstudie mit gesunden Probanden zur Beurteilung der technischen Umsetzung, der Wirkungen und 
Nebenwirkungen durchgeführt werden. Anhand der gewonnen Erkenntnisse sollten gegebenenfalls Modifikationen am Testsystem vorgenommen werden.

- Im zweiten Schritt war vorgesehen, anhand einer klinischen Evaluationsstudie an einer größeren Stichprobe gesunder Probanden und Patienten das modifizierte Testsystem hinsichtlich der technischen Umsetzung, der Wirkungen und Nebenwirkungen im klinischen Einsatz zu beurteilen.

\subsubsection{PRAKTIKABILITÄT DES TESTSYSTEMS}

Die Schnittstelle zwischen Benutzer und Testsystem ist charakterisiert durch das Eingabegerät, das visuelle Anzeigesystem und die Interaktionsmöglichkeit in der Aufgabensituation (s.a. Abb. 1.2.). Inwieweit die Testteilnehmer mit der vorgegeben Schnittstelle zurechtkamen, sollte in verschiedenen Konfigurationen untersucht werden.

(C.1) Untersuchung zur Benutzerbewertung der Praktikabilität des Testsystems und verschiedener VR-Anzeigesysteme (HMD, Projektionswand, Monitor) bei gesunden Probanden in einer präklinischen Pilotstudie und in einer klinischen Studie bei verschiedenen Patientengruppen.

Die Auswertung dieser Untersuchung sollte weitestgehend deskriptive Ergebnisse als Basis für zukünftige Fragestellungen liefern. Aufgrund des Fehlens empirischer Daten wurden keine Hypothesen aufgestellt. Die Ergebnisse der präklinischen Studie sollten zur Modifikation des Testsystems vor dem klinischen Einsatz genutzt werden. In der klinischen Studie sollten die Ergebnisse v.a. auf Unterschiede zwischen Patienten und Probanden untersucht werden.

\subsubsection{WIRKUNGEN VIRTUELLER REALITÄT}

Eine hohe Immersion und ein hoher Realismus sind als Zeichen einer guten VR-Realisation 'erwünschte Wirkungen' bei der Entwicklung des Testsystems. Der Einfluss des Anzeigesystems und des Testinhalts (=Testaufgabe) auf Immersion und Realismus sollte im Rahmen der neuropsychologischen Testung identifiziert werden.

(C.2) Untersuchung zur Benutzerbewertung von Realismus und Immersion.

Hypothese 1: Die Bewertung von Realismus und Immersion werden durch das eingesetzte VRAnzeigesystem und den Inhalt der Testaufgabe beeinflusst.

Hintergrund: Bereits die Bezeichnung und Zuordnung verschiedener Anzeigesysteme aufgrund des durch die VR-Darstellung eingenommenen Gesichtsfeldes in immersive (HMD, CAVE), semi-immersive (z.B. Einwand-Projektion) und non-immersive Systeme (Monitor) zeigt die allgemeine Einschätzung des Anzeigesystems als entscheidenden Faktor. Das Head Mounted Display bewirkt in der Regel eine gute Immersion und besitzt einen hohen Realitätsgrad bei der Navigation durch Einsatz eines Trackingsystems (Hendrix und Barfield 1996, Deisinger et al. 1997). Allerdings wird ebenso argumentiert, dass die Nebenwirkungen und Komfortprobleme, 
die durch den Einsatz eines HMD entstehen können, die Immersion verschlechtern. In Anlehnung an das 'Sense of Presence'-Konzept bewirkt ein besserer Realismus ein stärkeres Hineinversetztsein in die virtuelle Umgebung (Welch et al. 1996).

\subsubsection{NEBENWIRKUNGEN VIRTUELLER REALITÄT}

(C.3) Deskriptive Untersuchung zur Inzidenz und Art der Nebenwirkungen bei gesunden Probanden und verschiedenen Patientengruppen mittels Simulator Sickness Questionnaire.

Daten hinsichtlich des Auftretens unerwünschter Nebenwirkungen stammen zumeist aus militärischen Anwendungen und sind aufgrund des unterschiedlichen Inhalts auf das neuropsychologische VR-Testverfahren nicht übertragbar. Zum Zeitpunkt der Entwicklung des Testsystems existierten keine veröffentlichten systematischen Daten zum Auftreten von Simulator Sickness bei neurologischen, neuropsychologischen oder psychiatrischen VR. Anwendungen.

(C.4) Statistische Auswertung der Simulator-Sickness-Daten unter Berücksichtigung von beeinflussenden Faktoren.

Hypothese 2: Das Auftreten und die Ausprägung von Nebenwirkungen bei der neuropsychologischen VR-Testung werden durch individuelle (Alter, Geschlecht, Krankheit) und äußere Faktoren (verwendetes Anzeigesystem) beeinflusst.

Hintergrund: Daten aus der Forschung zur Simulator Sickness bei militärischen Anwendungen und insbesondere zur Motion Sickness legen nahe, dass individuelle Faktoren einen Einfluss auf das Auftreten von Symptomen haben. Mit steigendem Alter wird eine sinkende Inzidenz von Nebenwirkungen erwartet, im Geschlechtervergleich neigen Frauen eher zu Simulator Sickness (Kolasinski 1995). Inwiefern eine neurologische oder psychiatrische Erkrankung symptomverstärkend oder -mindernd ist, kann aufgrund fehlender Daten nicht beantwortet werden. Bekannt ist jedoch, dass Wahrnehmungsstörungen, Krankheit und Persönlichkeitsmerkmale Einfluss auf das Auftreten von Motion Sickness-Symptomen haben (Fox und Arnon 1988, Leimann Patt et al. 1988, Kolasinski 1995). Folglich kann vermutet werden, dass eine neuropsychiatrische Erkrankung ein eigenständiger Einflussfaktor in der vorliegenden Untersuchung ist. Das verwendete VR-Anzeigesystem wird als wesentlicher Faktor für das Auftreten von Simulator-Sickness-Symptomen gesehen (s.a. Kap. 1.2.3.3.), wobei kaum vergleichende Studien zwischen verschiedenen Anzeigemodalitäten existieren. 


\section{Methodik und Material}

Die folgenden Abschnitte zur Methodik beschreiben zeitlich und logisch aufeinander aufbauend die Phasen der Arbeit:

- Durchführung einer Literaturanalyse von existierenden medizinischen VR-Projekten zur Identifizierung verwendeter Technik zu Beginn der Laborplanung

- Planung eines medizinischen VR-Labors zur interdisziplinären Verwendung

- technische Entwicklung eines neuropsychologischen Testsystems als Projekt im VR-Labor

- Durchführung einer Evaluationsstudie als Teil der Testentwicklung

\subsection{Analyse medizinischer Virtual-Reality-Anwendungen}

Ein interdisziplinär nutzbares, medizinisches VR-Labor sollte den Bedürfnissen verschiedener Anwendergruppen gerecht werden. Dabei konnte aus wirtschaftlichen Gründen nur eine begrenzte Zahl an Geräten bereitgestellt werden, u.a. aufgrund der schnellen Systemwechsel bei ca. 1-jährigen Produktzyklen neuer Technologien. Um notwendige und ausreichende Basiskomponenten für eine interdisziplinäre Nutzung zu identifizieren, wurde vor der Konzeption des VR-Labors eine Literaturanalyse von existierenden medizinischen VirtualReality-Anwendungen unter dem Aspekt der technischen Realisierung durchgeführt: welche Komponenten wurden benutzt, welches Betriebssystem wurde verwendet und um welche Art von VR-Anwendung handelte es sich?

Für die Literaturanalyse wurden folgende VR-Anwendungen ausgewählt:

- Anwendungen, die als Publikationen in der elektronischen Datenbank MEDLINE 1996 bis Januar 1998 aufgeführt waren (National Library of Medicine des National Institute of Health, USA, verfügbar über http://www.ncbi.n/m.nih.gov). Als Suchbegriff wurde, virtual" (entsprechend ,virtual reality", ,virtual environments") verwendet.

- Anwendungen, die in den Proceedings des Kongresses ,Medicine Meets Virtual Reality 1996 und 1997 veröffentlicht wurden (Weghorst et al. 1996, Morgan et al. 1997).

Projekte und VR-Anwendungen, die in den o.g. Publikationen mehrfach auftraten, wurden nur einmal berücksichtigt. Ausgeschlossen wurden rein telemedizinische Anwendungen ohne VRCharakter (z.B. Telekonsultationen per PC), die dennoch häufig unter den Begriff der, Virtual Reality' summiert wurden.

Die Komponenten wurden nach der verwendeten Steuer- und Kontrollhardware, den visuellen Anzeigesystemen und den Eingabesystemen klassifiziert (nach Grimsdale 1992). Zusätzlich erfolgte eine Auflistung der verwendeten Betriebssysteme. 


\subsection{Aufbau und Organisation des VR-Labors}

An die Konzeption des VR-Labors wurden von der Planungsgruppe, bestehend aus Mitgliedern der Abt. Medizinische Informatik, Psychiatrie und Hämatologie/Onkologie, verschiedene Anforderungen gestellt:

- Das Labor sollte so eingerichtet werden, dass Projekte mit Partnern aus unterschiedlichen klinischen Disziplinen realisiert werden konnten. Daher sollten die VR-Komponenten vielfältig einsetzbar sein und gute Eigenschaften hinsichtlich Benutzerfreundlichkeit und unerwünschter Nebenwirkungen haben.

- Das Labor sollte in der Anschaffung deutlich unter $250.000 €$ liegen und ein Unterhalt durch laufende Mittel und zusätzliche Drittmitteleinwerbung möglich sein.

- Interdisziplinäre Projekte sollten Erkenntnisse zum medizinischen Einsatz von VRTechnologien liefern und daher Evaluationsphasen beinhalten.

- Eine dauerhafte Bindung von Ressourcen des VR-Labors durch Projekte sollte vermieden werden. Daher war beim klinischen Routineeinsatz eine weitgehende Unabhängigkeit vom VR-Labor anzustreben.

- Das Labor sollte in die vorhandene Datenverarbeitungs-Struktur eingebettet werden können und für spezifische Projekte erweiterbar sein.

Ein organisatorisches Ablaufschema sollte die Rahmenbedingungen zur Projektarbeit vereinheitlichen.

Die Komponenten des VR-Labors sollten aufgrund verschiedener Merkmale ausgewählt werden: für die Auswahl der Steuer- und Kontrollrechner waren Leistungsfähigkeit, Preis und die Einbettung in die Datenverarbeitungsstruktur des Universitätsklinikums Göttingen wesentliche Kriterien. Für die Eingabesysteme standen vor allem eine vielseitige Verwendbarkeit im Vordergrund. Bei den visuellen Anzeigesystemen gab es zahlreiche Kriterien gegeneinander abzuwägen: Preis, Benutzerfreundlichkeit, Immersionsgrad, Anzeigequalität, Vielseitigkeit und benötigter Steuerrechner.

Für die Auswahl der Komponenten wurden die in Kapitel 1.2.2. (Tab. 1.1. und 1.2.) beschriebenen Daten herangezogen: die Analyse bestehender medizinischer VR-Anwendungen sollte für bestimmte Anwendungsarten und Einsatzfelder essentielle Geräte identifizieren. 


\section{3. Überblick: Testentwicklung und Evaluation}

Die Neuentwicklung und Evaluation eines Virtual-Reality-gestützten neuropsychologischen Testsystems basierte auf Techniken und Methoden aus verschiedenen Fachgebieten:

- Die Art der Aufgabenstellung wurde anhand existierender neuropsychologischer Verfahren, Konzepte und Hypothesen entworfen und bestimmt.

- Die Planung und Realisierung eines computergestützten Testsystems erforderte Methoden aus den Computerwissenschaften.

In den Computerwissenschaften werden unter den Begriffen ,Systementwicklung' bzw. ,Software Engineering، Methoden angeboten, die eine systematische Erstellung von Computeranwendungen unterstützen. Diese Methoden sind eingeführt worden, um EDV. Anwendungen in planbaren und überschaubaren Schritten zu entwickeln und somit Fehlerquellen zu reduzieren, die Zuverlässigkeit zu verbessern und gleichzeitig ökonomisch vorzugehen (Pagel und Six 1994).

In der vorliegenden Arbeit wurde die Methodik der Testentwicklung in Anlehnung an die Planungs- und Realisationsschritte bei der Entwicklung von EDV-Systemen gegliedert. Grundsätzlich werden bei der Systementwicklung folgende Phasen unterteilt (modifiziert nach Zelkovitz 1979, Saranummi 1995, Birrel und Ould 1986):

- Analyse und Definition: Bestimmung des Funktionsumfangs und der Realisierungsanforderungen

- Entwurf: $\quad$ Erstellung der inneren Struktur des Systems mit Definition der Module und Komponenten

- Implementation: Umsetzung des Entwurfs zu einem Programm

- Test und Evaluation: Probeeinsatz, Testung auf Benutzbarkeit und Fehler

- Einsatz und Wartung: Routineeinsatz, Wartung, Erweiterung, Fehlerbehebung

Um Unsicherheitsfaktoren bei der Neuentwicklung eines Testsystems mit einer weitgehend neuartigen Technologie wie der Virtual Reality zu begegnen, ist bei der Entwicklung und Projektplanung auf ein sogenanntes ,Phasenmodell mit Rapid Prototype-Phase“ zurückgegriffen worden (Abb. 2.1.). Um unscharfe oder unklare Anforderungen an das System vor der Entwurfs- und Implementationsphase zu beheben, wurde nach der Analyse und Definition ein Prototyp mit Kernfunktionen entwickelt. Aufgrund einer praktischeren und realistischeren Einschätzung des Anforderungsprofils konnten so Änderungen am Funktionsumfang vorgenommen werden (s.a. Kap. 2.3.2./2.3.3.). 
Neuropsychologische Inhalte (z.B. Operationalisierung) stellten in fast allen Phasen der Systementwicklung ein wesentliches Element dar, lediglich in der Implementationsphase wurden allein programmiertechnische Aspekte berücksichtigt.

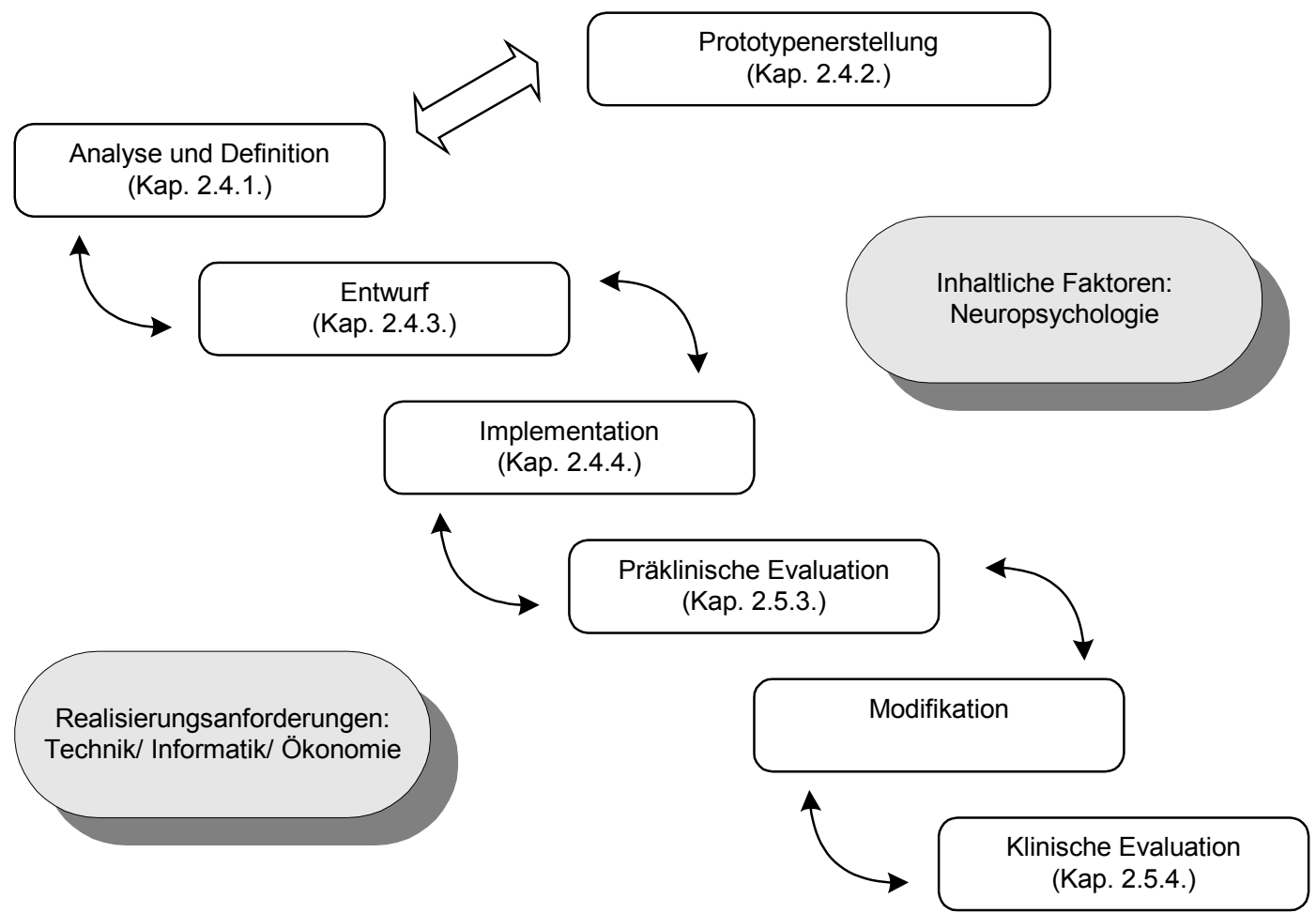

Abb.2.1. Um Prototypenerstellung erweitertes Phasenmodell der Systementwicklung (modifiziert nach Pagel u. Six 1994, S. 66). 


\subsection{Entwicklung des Neuropsychologischen Testsystems}

\subsubsection{Analyse und Definition}

\subsubsection{ANALYSE UND DEFINITION: NEUROPSYCHOLOGISCHE VIRTUAL-REALITY-TESTUMGEBUNG}

Allgemeine Beschreibung der erforderlichen Funktionen: Die neuropsychologischen Tests sollten mit Hilfe von Virtual-Reality-Aufgabenstellungen realisiert werden. Dabei sollten verschiedene kognitive Leistungen der Probanden getestet werden. Um einen qualitativen Unterschied zu Paper \& Pencil-Verfahren zu bieten, sollte ein möglichst hoher Realismus der Aufgabensituation gewährleistet werden. Die Handlungen der Testpersonen sollten so erfasst werden, dass diese hinsichtlich quantitativer Leistungsmerkmale ausgewertet werden konnten. Im Folgenden werden einzelne Aspekte zur Funktionalität und die theoretischen Hintergründe aufgeführt.

Die Operationalisierung neuropsychologischer Funktionen mit der Testumgebung sollte in der ersten Version folgende Lernaspekte umfassen:

1. visuell-räumliches Lernen in einer objektarmen Umgebung („Labyrinth“, egozentrische Repräsentation räumlichen Wissens)

2. topographisch-räumliches Lernen in einer objektreichen Umgebung („Landschaft“, allozentrische Repräsentation räumlichen Wissens)

Die Realisierung einer Testaufgabe zu exekutiven Funktionen war zu einem späteren Zeitpunkt vorgesehen. Die theoretischen Grundlagen zu den neuropsychologischen Konzepten sind in Kapitel 1.3. dargestellt worden. Für Navigationsaufgaben und topographisch-räumliches Lernen wurden bei Virtual-Reality-Anwendungen unterschiedliche Umgebungen verwendet: Gebäude (Ruddle et al. 1998), Labyrinthe (Maguire et al. 1998), Landschaften (Gillner und Mallot 1998), Städte (Aguirre und d'Esposito 1997), aber auch Adaptationen der in Tierexperimenten benutzten ,Morris Water Task، (Astur et al. 1998, Sandstrom et al. 1998).

Für die Testung sollte als objektarme Umgebung ein Labyrinth konstruiert werden, das bis auf die umgebenden Wände, Kreuzungen und Sackgassen keine Orientierungshilfen besaß. Demgegenüber sollte ein naturalistischer Park zur Verfügung stehen, in dem zahlreiche Objekte als Landmarken zur Orientierung dienen konnten. Die zugrunde liegenden Wegenetze für Labyrinth und Park sollten einen ähnlichen Schwierigkeitsgrad besitzen.

Ein wesentlicher Vorteil der Virtual-Reality-Testumgebung gegenüber bestehenden Testverfahren sollte eine hohe ökologische Validität sein (Pugnetti et al. 1995a, Rizzo et al. 1998a/b). Diese sollte vor allem durch eine realistische Testsituation geschaffen werden: der 
Benutzer sollte in ein virtuelles Labyrinth bzw. in eine virtuelle Landschaft hineinversetzt werden. Mit Durchlaufen der Wegenetze sollte der Benutzer aus einer aktiven Rolle heraus räumliches Wissen erwerben. Im Vergleich dazu kann bei Paper \& Pencil-Tests kein Positionsund damit Perspektivenwechsel vorgenommen werden, ein optischer Fluss fehlt (rein visuelles Lernen) und die reine 2D-Darstellung besitzt keine eigentlichen räumlichen Eigenschaften. Bei passiven Videopräsentationen zum Erwerb von topographischem Wissen kann die Testperson die Navigation nicht selbst steuern.

Wichtige technische Faktoren einer realistischen VR-Testsituation sind eine hohe visuelle Qualität, eine intuitiv verwendbare Benutzerschnittstelle und Echtzeitverhalten (Kaltenborn und Rienhoff 1993).

Die visuelle Qualität wird durch das Anzeigeverfahren und die Gestaltung der virtuellen Umgebung bestimmt. Die gängigen Anzeigeverfahren sind im Einleitungsteil (Tab. 1.1.) dargestellt. Kein Anzeigeverfahren konnte zum Zeitpunkt der Konzeption in der Summe möglicher Bewertungskriterien - räumliche Auflösung, Benutzerfreundlichkeit, Preis/ Leistungsverhältnis, Anpassung an physiologische Bedingungen - eindeutig favorisiert werden (Johnson et al. 1998, Deisinger et al. 1997). Die Eignung verschiedener Anzeigeverfahren zur neuropsychologischen Testung war deshalb Bestandteil der Evaluation innerhalb des vorliegenden Projekts. Die virtuelle Umgebung sollte hinsichtlich der optischen Gestaltung einen ausreichenden bildlichen Realismus besitzen, der es der Testperson ermöglichte, sich in die Situation hineinzuversetzen.

Die Benutzerschnittstellen für die Navigation in der Umgebung sollten mit ausreichender Funktionalität einfach bedienbar sein, da keine Computer-oder VR-Erfahrungen bei den Benutzern vorrausgesetzt werden konnte (Rizzo et al. 1998a). Eine sehr einfach erlernbare Navigation innerhalb eines festgelegten Wegenetzes kann mit zwei Handlungsarten erfolgen: (1) Bestimmung der Blickrichtung durch Drehen der Körperachse/ des Kopfes und (2) Starten der Laufbewegung zum nächsten Wegepunkt (s.a. Gillner und Mallot 1998).

Die auszuwählende Benutzerschnittstelle kann grundsätzlich nicht unabhängig vom visuellen Anzeigeverfahren gesehen werden: wird ein HMD verwendet, so geschieht die Drehung über Veränderung der Kopfposition mit einem Trackingsystem. Bei Projektionsverfahren oder Bildschirmpräsentation müssen andere Benutzerschnittstellen verwendet werden: 3D-Mäuse, Joysticks oder Tastatur. Da keine Bewegung im dreidimensionalen Raum, sondern lediglich in der Ebene notwendig ist, ist eine 3D-Maus mit komplizierter Handhabung ungeeignet. Sowohl Joysticks als auch eine Tastatur bieten sich für Drehung und Initiierung des Laufens an (z.B. Gillner und Mallot 1998, Sandstrom et al. 1998, Astur et al. 1998)

Die Echtzeit-Interaktivität war ein wichtiger Aspekt der Systementwicklung: entstehen zwischen einer Handlung des Benutzers und der Reaktion des VR-Systems auf die Handlung 
wahrnehmbare Verzögerungen, so wirkt sich dies negativ auf den "Sense of Presence aus (Welch et al. 1996). Daneben wird vermutet, dass diese Verzögerungen ein Cofaktor für die Entstehung der Simulator Sickness sind (Lewis und Griffin 1997). Daher sollten bei dem Testsystem für den Benutzer keine wahrnehmbaren Pausen zwischen Interaktion und Perspektivenwechsel entstehen.

Beim VR-System sollten durch Erfassung der Navigationsleistung die Aktionen soweit aufgezeichnet werden, dass die Navigation prinzipiell rekonstruiert werden konnte. Zeit, Ort und Position der Testperson in der virtuellen Umgebung sollten erfasst werden. Diese Rohdaten sollten archiviert werden, um mit dem Auswertungsmodul Leistungsparameter ermitteln zu können.

Das Testsystem sollte dahingehend entwickelt werden, dass auf der technischen Ebene Risikofaktoren zur Entstehung von Simulator-Sickness-Symptomen minimiert werden konnten. Allgemein kann die Navigation des Benutzers in der virtuellen Umgebung passiv (kein Einfluss auf die Navigation), aktiv (alle Freiheitsgrade vorhanden) oder aktiv-passiv (eingeschränkte Freiheitsgrade) geschehen. In einer Studie zeigte die aktiv-passive Form, die zwar nicht alle Drehungen und Bewegungen erlaubt, aber eine für die Aufgabe ausreichende Navigationsmöglichkeit bot, deutlich geringere Scores im Simulator Sickness Questionnaire als die rein aktive oder rein passive Navigation (Stanney und Hash 1998). Daher wurde die aktivpassive Form für die Benutzernavigation verwendet. Zwischen Handlung (z.B. Kopfdrehung) und Effekt (neue Blickrichtung) durfte keine wahrnehmbare Pause entstehen (Hettinger und Riccio 1992). Als akzeptabel wird im allgemeinen eine Interaktionspause von 100-125 ms betrachtet, für zeitkritische VR-Anwendungen wie Flugzeugsimulationen weniger als $35 \mathrm{~ms}$ (Pausch et al. 1992). Da die Messung der Interaktionspause methodischen Schwierigkeiten unterliegt, sind Orientierungswerte anhand der Bildwiederholungsrate vorgeschlagen worden. Diese soll stets größer als 10 Bilder pro Sekunde sein (Bryson 1995).

Im Hinblick auf das Benutzerprofil der primären Anwender des neuropsychologischen Testsystems (Patienten) durften keine Computer-oder VR-Kenntnisse angenommen werden. Neurologischen und psychiatrischen Patienten sollte eine Benutzerschnittstelle zur Verfügung gestellt werden, die einfach und schnell zu erlernen war. Diese sollte gewährleisten, dass der Patient sich ohne Einschränkung auf die Testsituation konzentrieren konnte.

Das VR-System sollte zur Evaluationsunterstützung flexibel mit verschiedenen technischen Konfigurationen (HMD, Projektionsverfahren, Shutter-Brillen) einsetzbar sein, um eine Evaluation der VR-Komponenten für medizinische Anwendungen zu gewährleisten. Die initiale Entwicklung und Evaluation des neuropsychologischen Testsystems sollte im VR-Labor ohne die Notwendigkeit einer zusätzlichen Gerätebeschaffung durchgeführt werden. Für die klinische Evaluation und den Einsatz sollten rein anwendungsorientierte, kostengünstige Geräte ausgewählt werden. 


\subsubsection{ANALYSE UND DEFINITION: EDV-GESTÜTZTE TESTADMINISTRATION}

Die Testadministration sollte den praktischen Einsatz des Testsystems erleichtern und den schnellen Zugriff auf Kenndaten zur Evaluationsunterstützung ermöglichen. Die Funktionen sollten im einzelnen sein:

- Testpersonenverwaltung: zur Erfassung soziodemographischer Daten, Vergabe einer automatischen Testpersonen-ID, Adresse.

- Durchführung der Tests: Auswahl, Aufruf und Dokumentation der durchgeführten Tests

- Erfassung der Evaluationsdaten: Datenbankbasierte Fragebögen zur Erfassung von evaluationsrelevanten Informationen zu Nebenwirkungen, subjektiver Bewertung etc.

Eine separate Stammdatenverwaltung (z.B. für Testauswahl, Testleiter, vorgegebene Antwortmöglichkeiten bei Fragebögen) wurde nicht als notwendig erachtet.

\subsubsection{ANALYSE UND DEFINITION: AUSWERTUNG}

Beschreibung der erforderlichen Funktionalität: Diese Auswertungswerkzeuge sollten Standard-Leistungsparameter aus den erfassten Navigationsdaten ermittteln und in einer Datenbank personenbezogen speichern.

Leistungsmerkmale bei Labyrinthversuchen sind Gegenstand zahlreicher Studien mit Tieren und Menschen, wobei Tierversuche meist videogestützt von Computern ausgewertet werden, während für Menschen Testversionen am Computer existieren (z.B. Morrison und Gates 1988). Bei Labyrinthversuchen wird das Betreten von falschen Pfaden oder Sackgassen generell als Fehler gezählt (Hoplight et al. 1996), wobei bei direkter Rückkehr auf den korrekten Pfad keine weiteren Fehler addiert werden. Die benötigte Zeit wird in Tierversuchen nicht als akkurates Maß für die Leistung betrachtet (Hoplight et al. 1996, Grossmann und Skinner 1996), vielmehr ist der Lernzuwachs (Abnahme der Fehlerzahl) über mehrere Versuche das entscheidende Leistungskriterium (Denenberg et al. 1991, Hoplight et al. 1996). Neben Fehlerzahl und Lernzuwachs sollten weitere Variablen ausgewertet werden können, z.B. Präferenzen beim Abbiegen an einer Kreuzung oder das mehrfache Begehen des gleichen Fehlers (Denenberg et al. 1991).

\subsubsection{Testsystem-Prototyp}

Im Rahmen der Systementwicklung wurde die Erstellung eines 'rapid prototype' mit Kernfunktionen vorgesehen, um zu überprüfen, ob die anhand der Analyse und Definition angestrebte Funktionalität des Systems sinnvoll oder noch überarbeitungsbedürftig ist. 
Der Prototyp der VR-Testumgebung wurde im Zeitraum von Februar bis April 1998 mit der Software ,Division dVise، entwickelt. Mit diesem Prototyp konnte sich der Benutzer innerhalb eines einfachen Wegenetzes entweder frei bewegen (aktive Navigation) oder von Wegepunkt zu Wegepunkt gehen (aktiv-passive Navigation). Die Navigation war mit einer 3D-Maus oder Tastatur möglich. Als visuelles Ausgabesystem konnten ein Computerbildschirm im monoskopischen oder stereoskopischen Modus (Shutter-Brillen), Projektionsgeräte oder ein HMD ohne Trackingsystem verwendet werden. Die Navigationsdaten konnten aufgezeichnet werden.

Der Prototyp wurde von Arbeitsgruppenmitgliedern und Abteilungsmitarbeitern getestet, um erste Hinweise auf die Praktikabilität der Navigationsart sowie Verständlichkeit und Komplexität der neuropsychologischen Testaufgabe zu erhalten.

Die informelle Testung des Prototypen durch die Projekt- und Abteilungsmitglieder ergab folgende Ergebnisse:

- Visuelles Anzeigesystem: Alle Anzeigeverfahren wurden als sinnvoll bezeichnet, wobei den Projektionsgeräten wegen der größeren Anzeigefläche gegenüber dem Bildschirm der Vorzug gegeben wurde. Das HMD wurde wegen des Tragekomforts kritisiert. Der Vergleich von stereoskopischen zu monoskopischen Verfahren ergab, dass sich für die Testaufgabe kein subjektiver Vorteil mit dem räumlichen Eindruck der Shutter-Brillen ergab. Teilweise wurde das stereoskopische Verfahren als qualitativ schlechter empfunden, da die halbierte Bildwiederholungsrate häufiger zu Flackereffekten führte.

- Navigation: die aktive Navigation, bei der sich der Benutzer frei innerhalb des Weges bewegen konnte, wurde vor allem von unerfahrenen Computerbenutzern als sehr schwierig bezeichnet. Im Zusammenspiel von HMD und 3D-Maus verloren die Anwender schnell die Orientierung; dies produzierte z.T. heftige Übelkeit. Die aktiv-passive Navigation, bei der der Benutzer von Wegepunkt zu Wegepunkt, geführt‘ wird, wurde positiv bewertet. Abrupte Beschleunigungen führten auch bei der aktiv-passiven Navigation bei einigen Anwendern zu unerwünschten Nebenwirkungen wie Übelkeit und ermüdeten Augen. Dies konnte jedoch durch eine verlangsamte und sanfte Beschleunigung eliminiert werden.

- Entwicklungswerkzeuge: das Virtual-Reality-Entwicklungspaket dVise, das aus den Ingenieurwissenschaften stammt, erschien wegen unflexibler Programmierung und hohen Hardwareanforderungen bei mäßigen Leistungsdaten nicht geeignet für die weitere Entwicklung des Systems und wurde im folgenden durch die Entwicklungsumgebung ,Realimation' ersetzt.

Die Ergebnisse der Prototypenevaluation wurden in einem modifizierten Entwurf berücksichtigt. 


\subsubsection{Entwurf}

Die in Kap. 2.3.2. aufgeführten Funktionsbeschreibungen sind Grundlage des Entwurfs. Hierbei wird das gesamte Softwaresystem in sogenannte ,Module' gegliedert: aufgrund der Funktionsbeschreibungen werden Anwendungsteile, die eine für sich abgrenzbare Funktionalität besitzen, bestimmt. Diese können dann einfacher und übersichtlicher entworfen, implementiert und gepflegt werden.

Beim Funktionsumfang des Testsystems ließen sich gemäß der Definition die Virtual-RealityTestumgebung, die Testadministration und die Auswertung unterscheiden. Vom Anwendungscharakter handelte es sich im ersten Fall um eine Virtual-Reality-Anwendung mit Datenaufzeichnung, bei den letzteren um Datenbankanwendungen mit Datenverwaltung und Datenauswertung. Das Zusammenspiel der Module ist in Abbildung 2.3. (Seite 36) dargestellt.

\subsubsection{ENTWURF MODUL 1: VIRTUAL-REALITY-TESTUMGEBUNG}

Das VR-Modul sollte eine Testumgebung mit definierten Handlungsmöglichkeiten und Aufzeichnung der Handlung zur Verfügung stellen. Dabei sollte die Möglichkeit vorgesehen werden, verschiedene VR-Geräte zu benutzen. Dieses Modul sollte in folgenden Arbeitsschritten ablaufen: Start des VR-Systems bei variabler technischer Ausstattung (Initialisierung), Bereitstellung der interaktiven Testumgebung, dabei Aufzeichnung der Handlung. Daneben musste eine Schnittstelle zum Datenbanksystem hergestellt werden (siehe Abb. 2.2., Seite 34).

Bevor der Test starten konnte, mussten durch Initialisierung des Virtual-Reality-Systems verschiedene Informationen zur Verfügung gestellt werden: die geometrischen Daten der virtuellen Umgebung, Aufgabenstellung und Regeln in der virtuellen Umgebung und Kennwerte für die Verwendung der gewünschten VR-Geräte.

Geometrische Daten mussten als Drahtmodelle in einer Datei gespeichert werden. Die Navigationsmöglichkeiten und Regeln sollten teilweise im Programm, teilweise extern in Dateien festgelegt werden. Für prinzipielle Funktionen wie die Art der Navigation war die Implementation im Programm vorgesehen, variable Daten wie richtige und falsche Wege für eine bestimmte Testumgebung sollten in externen Dateien hinterlegt werden können.

Um ohne Eingriff in das Programm verschiedene visuelle Anzeigesysteme (z.B. HMD, ShutterBrillen, Monitor, Projektionssysteme) benutzen zu können, sollten auch deren Kennwerte (Auflösung, Farbtiefe, Darstellungsfrequenz, Mono-/ Stereoskopie) in einer externen Datei 


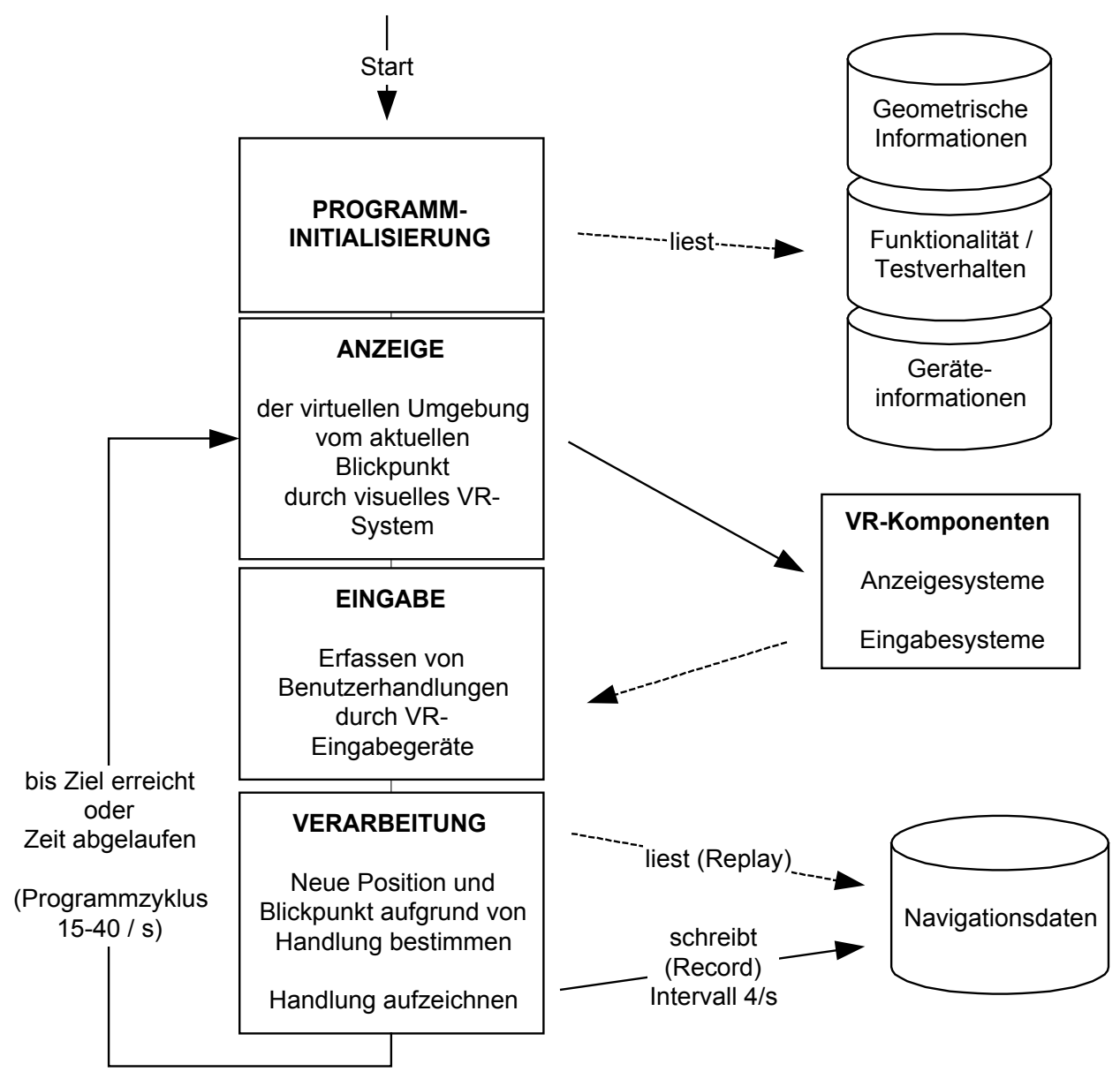

Abb. 2.2. Flussdiagramm der internen Arbeitsschritte im Modul, Virtual-Reality-System “

hinterlegt werden. Eine Unterstützung für Mehrwandprojektionssysteme, die vor Ort nicht vorhanden waren, wurde nicht vorgesehen.

Für die erste Version des Testsystems waren die Implementation von Tastatur, Joystick und Trackingsystem als Eingabegeräte vorgesehen.

Bei der Anzeige wird die virtuelle Umgebung vom aktuellen Blickpunkt des Benutzers aus als Bild dargestellt. Der Vorgang des Erstellens eines zweidimensionalen Bildes aus einer dreidimensionalen geometrischen Struktur von einem festgelegten Blickpunkt aus wird als ,Rendering“ bezeichnet. Bei der Anzeige mussten die Kenndaten des visuellen Ausgabesystems berücksichtigt werden.

In dem Teilsystem Eingabe wird auf eine Handlung des Benutzers gewartet. 
Allen Eingabesystemen sollte gemeinsam sein, dass man mit ihnen eine Rechtsdrehung, Linksdrehung und Auslösen des Geradeauslaufens von Wegepunkt (Kreuzung, Sackgasse) zu Wegepunkt realisieren konnte.

Bei Benutzung der Tastatur sollten die Aktionen mit den Pfeiltasten ausgelöst werden. Mit dem Joystick sollten die Drehungen mit dem Seitwärtsdrücken des Steuerknüppels ausgelöst werden, der Geradeauslauf mit Druck auf den Feuer-Knopf.

Vom Trackingsystem im Einsatz mit einem Head Mounted Display sollte lediglich der sogenannte yaw-Wert eingelesen werden (Drehung um die aufrechte Körperachse/ Z-Achse im kartesischen Koordinatensystem). Die Änderung des eingelesenen Winkels, resultierend aus der rechts/links Kopfdrehung des Probanden, sollte in einen Schwenk der Blickrichtung umgesetzt werden. Für die Initiierung des Geradeauslaufs war der Knopfdruck einer Maustaste vorgesehen.

Bei der Verarbeitung der Daten sollte die aktuelle Position der Testperson mit Zeitangaben und Fehlerinformation in die Navigationsdatenbank gespeichert werden. Anhand der Eingabedaten sollte die neue Position und Blickrichtung des Probanden berechnet werden.

Der Zyklus Anzeige - Eingabe - Verarbeitung sollte pro Sekunde häufiger als 15 mal durchgeführt werden. Bei einer Frequenz von größer als $25 \mathrm{~Hz}$ kommt es für den Betrachter zu einer absolut flüssigen Bewegung, $10 \mathrm{~Hz}$ werden als Minimum für VR-Anwendungen gesehen.

\subsubsection{ENTWURF MODUL 2: TESTADMINISTRATION}

Das Modul wurde als Datenbankprogramm konzipiert. Hier sollten Daten der Testpersonen aus Fragebögen erfasst und die Durchführung und Dokumentation der VR-Tests gesteuert werden. Die erfassten Daten sollten in einer Datenbank des VR-Testsystems abgelegt werden.

Mit der Testpersonen-Verwaltung sollte die Erfassung der soziodemographischen Daten mit einem Formular erfolgen, mit dem über eine automatische generierte Testpersonen-ID verknüpfte Informationen für jede Testperson einzusehen waren (z.B. bereits durchgeführte Tests).

Zur Unterstützung der Testdurchführung wurden folgende Funktionen vorgesehen: Generierung eines Eintrags mit Datum, Zeit und Testleiter für den Versuch; Auswahl der gewünschten Tests mit Unterstützung bestimmter VR-Komponenten aus einer vorgegebenen Liste; bei Durchführung des Tests Dokumentation über Zeitpunkt und Art des Tests für jeden Probanden. Zur Erfassung von Evaluationsdaten sollten elektronische Formulare der Fragebögen ,Prä-Test', ,Post-Test' und des ,Simulator Sickness Questionnaire' zur Verfügung stehen (Anhang 6.2., 6.4., 6.5). 


\subsubsection{ENTWURF MODUL 3: AUSWERTUNG}

Für jeden Versuchsdurchgang sollte eine Auswertung der Leistungsparameter und Speicherung der Leistungsdaten in der Datenbank durchgeführt werden. Folgende Leistungsmerkmale wurden für die Basisauswertung bestimmt:

- Zeit des Durchgangs,

- Erreichen des Ziels,

- Summe der Fehler, Entscheidungen, mit Entscheidungen verbrachte Zeit, Bewegungen auf korrektem Pfad und Bewegungen auf falschem Pfad.

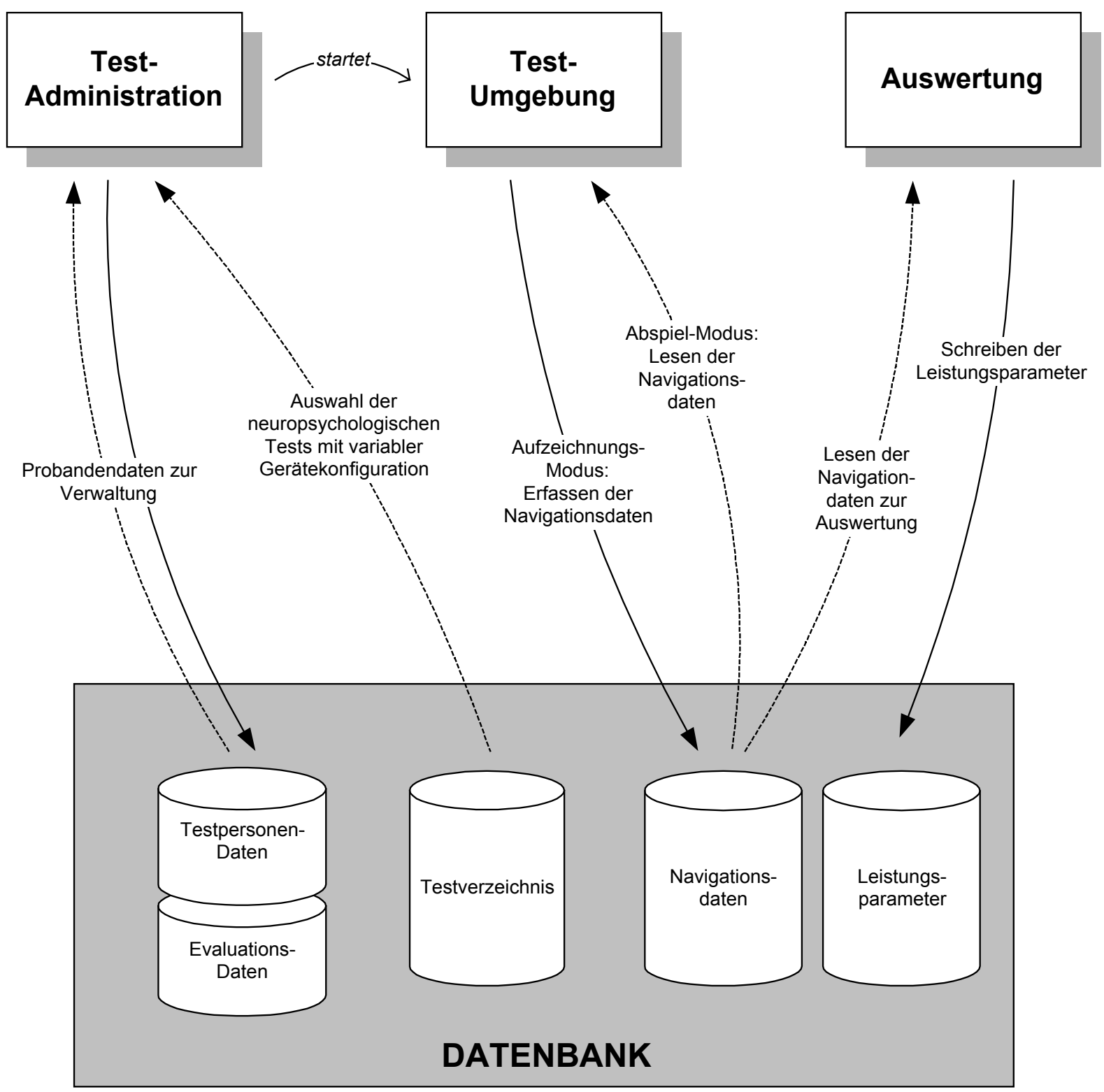

Abb. 2.3. Zusammenspiel der Module des Testsystems mit vereinfachtem Datenmodell 


\subsubsection{Implementation}

Bei der Implementation wurden auf Grundlage des Entwurfes die einzelnen Module programmiert und der technische Aufbau des Systems umgesetzt. Die dabei verwendeten Programme (Software) und technischen Komponenten (Hardware) sind tabellarisch mit Aufführung der Funktion angegeben (Tab. 2.1. und 2.2.).

Tab. 2.1. Verwendete Software zur Programmierung des Testsystems

\begin{tabular}{ll}
\hline Produkt & Aufgabe \\
\hline Blocks \& Materials & Vorgefertigte geometrische Objekte zur Benutzung in Kinetix 3D Studio Max \\
Borland Delphi & Datenbank-Entwicklungssystem zur Programmierung der Administration und \\
& Auswertungsroutinen. \\
Corel Photo Paint 7 & Texturenerstellung und -bearbeitung. \\
Division dVise & VR-Entwicklungsumgebung und Laufzeitbibliothek zur Entwicklung des, rapid \\
& prototype‘. \\
Kinetix 3D Studio Max R2 & Erstellung der statischen, geometrischen Modelle für die virtuelle Umgebung. \\
Microsoft C++ & C++-Entwicklungsumgebung zur Programmierung der VR-Anwendung unter Benutzung \\
& der Realimation VSG-Bibliothek. \\
Realimation VSG 4.4 & Optimierung der geometrischen Modelle und Laufzeitbibliothek für die VR-Anwendung. \\
\hline
\end{tabular}

Tab. 2.2. Verwendete Hardware bei der Entwicklung des Testsystems

\begin{tabular}{|c|c|}
\hline Produkt & Aufgabe \\
\hline Intergraph Workstation & Graphik-Workstation zur Entwicklung des VR-Systems. \\
\hline Polhemus 3SPACE FasTrak & $\begin{array}{l}\text { Tracking System zur räumlichen Erfassung der Kopfhaltung der Testperson. Polhemus } \\
\text { Incorporated, Colchester, Vermont, USA. }\end{array}$ \\
\hline Stereographics Crystal Eyes & $\begin{array}{l}\text { Stereoskopisches Brillensystem. Stereographics Corporation, San Rafael, California, } \\
\text { USA. }\end{array}$ \\
\hline Virtual Research V8 & $\begin{array}{l}\text { Head Mounted Display. Technische Spezifikation: Stereoskopisches Head Mounted- } \\
\text { Display auf LCD-Basis, farbfähig, Auflösung } 640 \text { x } 480 \text { Pixel. Virtual Research Systems } \\
\text { Inc., Santa Clara, California, USA. }\end{array}$ \\
\hline
\end{tabular}

Software und Hardware wurden nach Durchführung von Marktanalysen, Produktvergleichen und Testungen ausgewählt. Bei den Softwarekomponenten wurde besonders auf einen problemlosen Datenimport und -export von Graphikdaten zwischen den einzelnen Applikationen geachtet. Die Hardware wurde unter Berücksichtigung von Kosten, Leistungsfähigkeit, Schnittstellenfähigkeit sowie Einbindung in das Datenverarbeitungskonzept der Abteilung Medizinische Informatik ausgewählt. 


\subsection{Evaluation}

Die Evaluation des Testsystems hinsichtlich bekannter VR-bedingter Wirkungen und Nebenwirkungen sowie der Praktikabilität war in zwei Schritten vorgesehen (Abb. 2.1.):

- zunächst die Durchführung einer präklinischen (Pilot-)Evaluation, anhand deren Ergebnisse eine Modifikation des Testsystems durchgeführt werden sollte,

- im zweiten Schritt die Durchführung einer klinischen Evaluation, um an einer größeren Patienten- und Probandenstichprobe neuropsychologische und medizininformatische Fragestellungen zu beantworten.

\subsubsection{Erfassung erwünschter und unerwünschter VR-bedingter Effekte}

Zur Dokumentation von unerwünschten Nebenwirkungen ist von Kennedy und Lane der Simulator Sickness Questionnaire (SSQ) mit 16 Items erarbeitet worden (Kennedy und Lane 1993). Hierbei werden drei Symptomenkomplexe (Cluster) unterschieden: der 'oculomotor'-, 'nausea'- und 'disorientation'-Cluster. Der Fragebogen und die Berechnung der einzelnen Scores sind im Anhang aufgeführt (Anhang 6.2/6.3). Der Fragebogen wurde vor Testbeginn, nach Beendigung des VR-Testes und nach definierten Testabschnitten erhoben (siehe Abb. 2.4, S. 41; Abb. 2.5, S. 44), mit dem Ziel, Symptomverläufe zu dokumentieren. Zusätzlich zur Abfrage der einzelnen Testitems wurden die Patienten nach jedem Testdurchgang auf Unwohlsein befragt, um gegebenfalls frühzeitig Pausen einzulegen oder die VR-Testung abzubrechen.

Zu den erwünschten VR-Effekten gehört die 'Immersion', das Gefühl des VR-Benutzers, sich in die virtuelle Umgebung hineinzuversetzen. Die Probanden wurden nach Durchführung der VRTestung schriftlich dahingehend befragt (Anhang 6.5 - Frage 5, Anhang 6.6. - Frage 10).

\subsubsection{Bedienbarkeit und Beurteilung des Testsystems durch die Testperson}

Zur Bewertung der technischen Realisation des neuropsychologischen Testsystems wurden die Probanden nach der Testdurchführung neben der Immersion zur Realitätsnähe und - abhängig vom Versuchsdesign - zur Präferenz verschiedener Anzeigeverfahren befragt (Anhang 6.5 Frage 4/8, Anhang 6.6. - Frage 9).

Zur Bewertung der hier benutzten aktiv-passiven Navigationsart (s.a. Kap. 2.3.2.) mussten sich die Probanden nach der Testung zu Problemen bei Navigation und Handhabung äußern (Anhang 6.5 - Frage 2, Anhang 6.6. - Frage 6). 


\subsubsection{Stichprobe, Material und Testdurchführung der präklinischen Studie}

\subsubsection{AUSWAHL DER STICHPROBE}

Studenten bei Seminaren der Abteilung Psychiatrie/Schwerpunkt Neuropsychologie sowie Mitarbeiter innerhalb der Abteilungen Psychiatrie/Schwerpunkt Neuropsychologie und Medizinische Informatik wurden über die Möglichkeit der Teilnahme an einer Pilotstudie zu Hirnleistungstests mittels Virtual-Reality-Technik informiert. Zweiundzwanzig projektfremde Probanden wurden ausgewählt.

Als Ausschlusskriterium wurde das Vorliegen neurologisch-psychiatrischer und anderer Erkrankungen festgelegt, die eine Gefährdung des Probanden darstellen konnten, sowie ein Alter über 60 Jahre.

\subsubsection{MATERIAL}

Der Piloteinsatz war initial im technischen Rahmen der Basisausstattung des VR-Labors vorgesehen, doch wurde für die Evaluation ein für die Testung günstigerer Einsatzort ausgewählt. VR-Komponenten wurden bei Bedarf aus dem Labor verwendet. Im Rahmen einer Erweiterung der technischen Ausstattung wurden mit dem neuropsychologischen Testsystem Leistungstests verfügbarer Computersysteme durchgeführt. Dabei wurden der Park (34685 Dreiecksflächen) und das Labyrinth (6345 Dreiecksflächen) als VR-Umgebung verwendet, um die Bildwiederholungsrate bei Navigation zu messen. Als zusätzlicher Steuerrechner für den Einsatz bei der Evaluationsstudie wurde ein Doppelprozessor-PC mit zwei leistungsfähigen Graphikkarten (Spezifikation: siehe Anhang 6.13.) angeschafft.

Bei der technischen Evaluation wurden exemplarisch zwei Anzeigeverfahren herausgegriffen:

- Head Mounted Display (Virtual Research HMD V8 Virtual Research Corp., Santa Clara, USA, 2 LCD-Bildschirme mit einer Auflösung von 640 x 480 Pixel) im monoskopischen Modus mit Head-Tracking-System (Polhemus FasTrak) und Standardmaus zur Initiierung von Aktionen (Kap. 1.2., Abb. 1.4.).

- monoskopische Ein-Wand-Projektion mit LCD-Projektor (Davis DL450 projector, ASK/Infocus ASA, Fredrikstad, Norwegen, Auflösung: $800 \times 600$ Pixel) und einer Projektionsfläche von $\sim 2 \times 1.60$ m mit Standardtastatur als Eingabegerät.

Für das HMD resultiert ein durch die Anzeige abgedecktes Gesichtsfeld (FOV) von etwa 60 diagonal, ähnliche Werte ergeben sich für das Projektionssystem bei einer Position des Probanden etwa $2 \mathrm{~m}$ vor der Projektionsfläche. 


\subsubsection{TESTDURCHFÜHRUNG}

Der Testablauf erfolgte anhand eines festgelegten Protokolls, das vereinfacht in Abb. 2.4. (S. 41) zu sehen ist. Vor Beginn des Tests wurden die Probanden mittels eines standardisierten Textes über die Art des Versuches und mögliche Nebenwirkungen informiert (Anhang 6.7.) und bei Fragen mündlich beraten. Bei Einverständnis zur Teilnahme am Test (Anhang 6.9.) wurde ein Fragebogen zu soziodemographischen Daten, Sehproblemen, Computerkenntnissen, Vorerkrankungen und Anfälligkeit für Kinetosen ausgefüllt (Anhang 6.4.). Alle Daten wurden mit einer ID versehen und anonymisiert aufgezeichnet. Der Proband/ die Probandin wurde der Versuchsbedingung ,Park' oder ,Labyrinth“ randomisiert zugewiesen (klassifiziert nach Geschlecht). Vor der Einweisung in die Bedienung des VR-Systems wurde der erste Simulator Sickness Questionnaire (SSQ) ausgefüllt.

Anhand eines standardisierten Textes wurden die Probanden in die Bedienung des VR-Systems und die Aufgabenstellung eingewiesen (Anhang 6.10). Mit einer Trainingsaufgabe, die der späteren Aufgabenstellung ähnelte, hatten die Probanden sechs Minuten Zeit, die Bedienung des Systems im Projektionsmodus zu erlernen. Nach dem Trainingsdurchgang wurde erneut ein SSQ erhoben und gefragt, ob der Proband eine Pause oder den Versuch abbrechen möchte. Im Folgenden wurden zunächst 5 Versuchsdurchgänge im Projektionsmodus durchgeführt, für die die Probanden jeweils 6 Minuten Zeit hatten. Nach jedem Durchgang wurden die Probanden gefragt, ob sie eine Pause einlegen oder den Test abbrechen wollten. Nach dem letzten Durchgang im Projektionsmodus wurde wieder ein SSQ ausgefüllt und eine 10minütige Pause angeboten. An die Durchgänge im Projektionsmodus wurden 6 Durchgänge im HeadMounted-Displa+y (HMD)-Modus angeschlossen. Die Aufgabenstellung war identisch, aber mit einem anderen Wegenetz als Grundlage. Wieder wurde dem Benutzer die Funktion des Systems erklärt und ein Trainingsdurchgang durchgeführt. Das weitere Procedere entsprach dem Vorgehen beim Projektionsmodus.

Nach Durchführung aller 12 Testdurchgänge und auch bei vorzeitigem Abbruch wurde ein Fragebogen zur Benutzerbewertung des Testsystems von den Probanden ausgefüllt (Anhang 6.5.) und ein Abschlussgespräch geführt. 


\section{Vorbereitung}

Aufklärung und

Einverständnis

Randomisierung in
Gruppe PARK
Gruppe LABYRINTH

\section{Testabschnitt 'Projektionsmodus'}

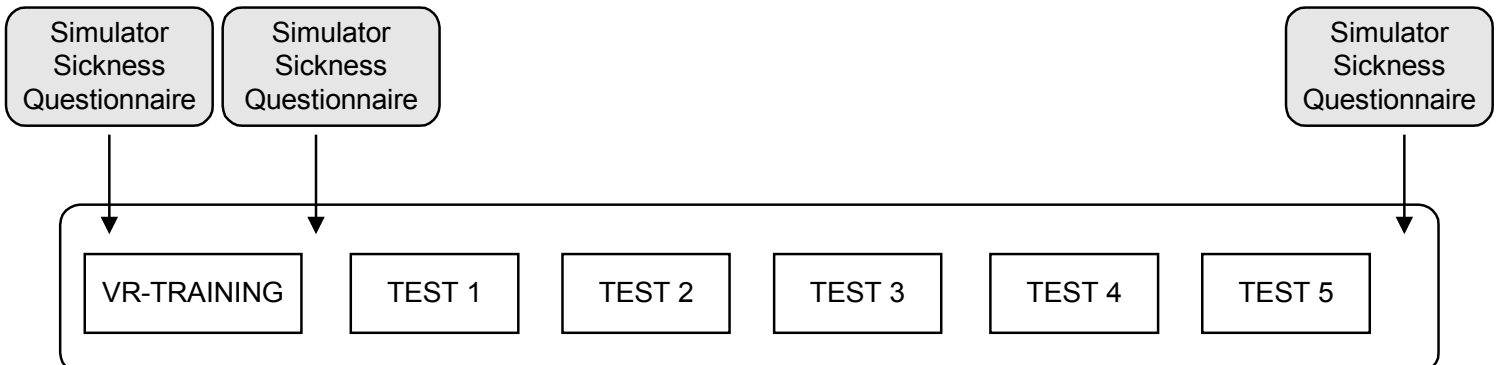

3. Testabschnitt 'Modus Head Mounted Display'

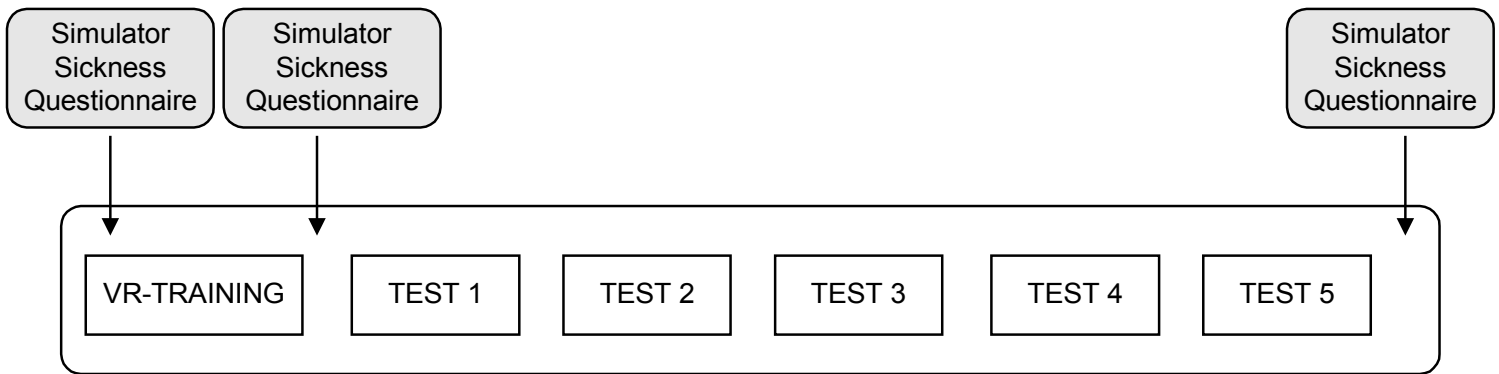

\section{Abschlussbewertung / -gespräch}

Fragebogen POST-TEST

Abb. 2.4. Schematische Darstellung des Versuchsablaufs der präklinischen Evaluation 


\subsubsection{Stichprobe, Material und Testdurchführung der klinischen Studie}

\subsubsection{AUSWAHL DER STICHPROBE}

- Patienten mit supratentoriellen ischämischen oder hämorrhagischen Läsionen

Es wurden Patienten mit umgrenzten und unilateralen vaskulären Läsionen im Bereich des Frontal-, Parietal- und des Temporallappens, welche in der Neurologischen Abteilung der Universitätsklinik Göttingen behandelt wurden, untersucht. Die Untersuchung erfolgte ca. 3 Monate nach Entlassung.

- Patienten mit medikamentenresistenter Frontal-oder Temporallappenepilepsie (prä-und postoperativ)

Es wurden Patienten mit medikamentenresistenter Frontal-oder Temporallappenepilepsie, die in der Neurochirurgischen oder Neurologischen Abteilung der Universitätsklinik Göttingen in Behandlung waren, untersucht. Die prächirurgischen Patienten befanden sich in der Regel zur neurophysiologischen Diagnostik des epileptogenen Focus in der stationären Behandlung, die Gruppe der post-operativen Epilepsiepatienten befand sich in der ambulanten Nachbehandlung nach Durchführung einer OP.

\section{- Patienten mit der Verdachtsdiagnose einer Demenz}

Patienten mit der Verdachtsdiagnose einer Demenz vom Alzheimer Typ oder einer vaskulären Genese, sowie ätiologisch ungeklärten Formen wurden von der Gedächtnissprechstunde der Psychiatrischen Abteilung der Universitätsklinik Göttingen sowie von niedergelassenen Nervenärzten zur Abklärung der kognitiven Defizite überwiesen. Aufgrund der Ergebnisse im zunächst durchgeführten MMSE (Mini-Mental State Examination) wurden nur diejenigen Patienten in die weitere Untersuchung eingeschlossen, bei welchen keine schweren kognitiven Beeinträchtigungen vorlagen (MMSE > 24 Rohpunkte) und die rechtliche Einwilligungsfähigkeit zur Untersuchung gegeben war.

\section{- Gesunde Kontrollprobanden}

Zur Bestimmung des normalen neuropsychologischen Leistungsniveaus in den virtuellen Testsystemen und als Referenzgruppe der technischen Evaluation wurden gesunde Kontrollprobanden untersucht. Diese wurden teilweise aus nicht-neurologischem Patientengut der Universitätsklinik akquiriert, teilweise über Aushänge und Annoncen. Ein sample-to-sample matching für die neuropsychologische Auswertung war vorgesehen.

\section{- Ausschlusskriterien}

a. Ausgeschlossen wurden Patienten bei Vorliegen von Zweiterkrankungen mit ZNS. Beteiligung, die neuropsychologisch relevante Auswirkungen haben konnten. 
b. Da eine mögliche Nebenwirkung der VR-Umgebung die Induktion von Übelkeit und Schwindel sein kann, wurden Patienten, bei welchen diese Symptome vorlagen, ausgeschlossen.

c. Für alle Patientengruppen mit Ausnahme der Demenzgruppe (Einschluss bis 80 Jahre) galt ein Lebensalter $>75$ als Ausschlusskriterium.

\subsubsection{MATERIAL}

Drei Anzeigekonfigurationen standen den Teilnehmern der klinischen Studie zur Verfügung:

- $\quad$ ein kommerzielles HMD (Sony Glasstron, 2 LCD-Bildschirme mit einer Auflösung von $800 \mathrm{x}$ 255, $\sim 35^{\circ}$ diagonaler FOV, Gewicht 180g) mit Head-Tracking-System (Intersense Intertrax). Dieses stand in der präklinischen Studie nicht zur Verfügung, die Gründe für den Wechsel des HMD werden im Ergebnisteil aufgeführt (Kap. 3.4.6.).

- $\quad$ ein Standard-21-Zoll-Monitor

- $\quad$ eine Ein-Wand-Projektion (wie in 2.5.3.2 beschrieben).

Der Doppelprozessor-PC wurde als Steuerrechner von der präklinischen Studie übernommen.

\subsubsection{TESTDURCHFÜHRUNG}

Der Testablauf wurde durch ein Protokoll festgelegt (Abb. 2.5.). Dies unterschied sich vom präklinischen Ablauf vor allem in der durchgehenden Verwendung einer Anzeigemodalität pro Proband. Vor dem Beginn des Tests wurden die Probanden mittels eines standardisierten Textes über die Art des Versuches und mögliche Nebenwirkungen informiert (Anhang 6.8.).

Bei Teilnahme wurde ein Fragebogen zu soziodemographischen Daten, Vorerkrankungen und Anfälligkeit für Kinetosen ausgefüllt, weiterhin wurden abhängig von der Gruppe krankheitsspezifische neuropsychologische Untersuchungen durchgeführt (z.B. Lateralität bei Epilepsie, Tests zum visuellen Lernen). Alle Daten wurden mit einer ID versehen und anonymisiert aufgezeichnet.

Allen Gruppen wurden die drei Anzeigesysteme (HMD, Projektion, Monitor) erklärt. Anhand initialer Beobachtungen - bei denen ältere Patienten eine deutliche Zurückhaltung oder Unsicherheit bei der Verwendung des HMD zeigten - wurde den Testteilnehmern die Auswahl wie folgt empfohlen: Schlaganfall- und Demenzpatienten wurden Monitor oder Projektion empfohlen; Epilepsie-Patienten und gesunden Probanden wurde vorgeschlagen, das HMD zu verwenden, jedoch bestand auch die Möglichkeit der Verwendung von Monitor oder Projektion.

Anhand eines standardisierten Textes wurden die Probanden in die Bedienung des VR-Systems und die Aufgabenstellung eingewiesen. Mit einer Trainingsaufgabe, die der späteren 


\section{Vorbereitung/ non-VR-Tests}
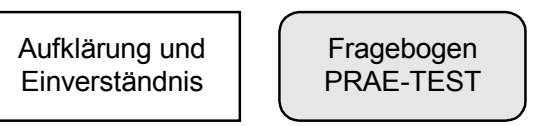

Auswahl visuelles

Anzeigesystem (HMD,

Monitor, Projektion)

\section{VR-Testung}

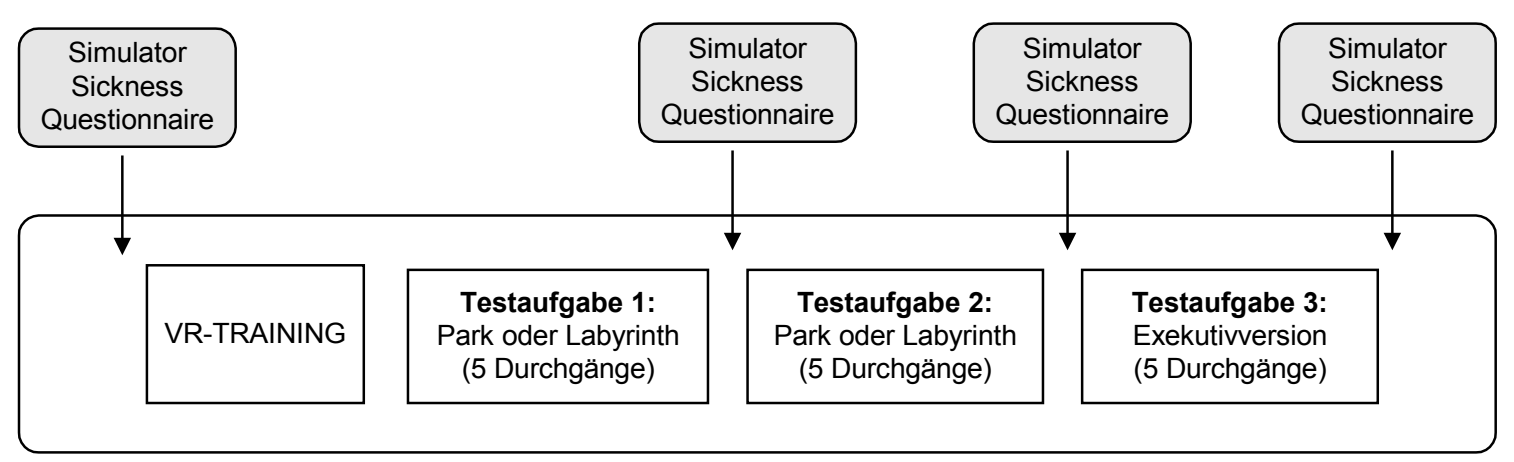

\section{Abschlussbewertung / -gespräch}

\section{Fragebogen Nachbefragung}

\section{Abb. 2.5. Schematische Darstellung des Versuchsablaufs der klinischen Evaluation}

Aufgabenstellung ähnelte, hatten die Probanden bis zu fünf Minuten Zeit, die Bedienung zu erlernen. Maximal drei Testaufgaben (Park, Labyrinth, Exekutiv) mit je fünf Testdurchgängen wurden durchgeführt. Die Testteilnehmer begannen entweder mit dem Park oder dem Labyrinth, beide Aufgaben sollten durch alle Testteilnehmer durchgeführt werden. Ließ die Konzentrationsspanne dies zu, wurde zusätzlich die Aufgabe zu exekutivem Lernen bearbeitet.

Ein Simulator Sickness Questionnaire (SSQ) wurde vor der ersten sowie nach jeder weiteren Testaufgabe durchgeführt, insgesamt also höchstens vier Mal. Nach Durchführung der Testdurchgänge, auch bei vorzeitigem Abbruch, wurde ein Fragebogen zur Benutzerbewertung des Testsystems von den Probanden ausgefüllt (Anhang 6.6.).

\subsubsection{Datenanalyse}

Die Auswertung der Daten erfolgte mit dem Statistikprogrammpaket SPSS (SPSS 2000). Hierzu wurden u.a. Daten der erfassten Simulator Sickness Questionnaires und Leistungsparameter aus dem Testadministrationsprogramm übernommen. Neben deskriptiven statistischen Verfahren wurden auch korrelations-, varianz- und kovarianzanalytische Verfahren durchgeführt. 


\section{Ergebnisse}

Dieses Kapitel stellt die Ergebnisse dreier Arbeitsabschnitte dar:

Analyse von existierenden medizinischen VR-Anwendungen unter technischen Aspekten,

Organisationsform und Aufbau des VR-Labors,

Entwicklung und Evaluation des neuropsychologischen VR-Testsystems.

Diese drei Abschnitte bauen inhaltlich aufeinander auf: der Aufbau des VR-Labors basierte auf den Erkenntnissen der technischen Analyse. Die Entwicklung des neuropsychologischen Testsystems wurde im organisatorischen und technischen Rahmen des VR-Labors durchgeführt.

\subsection{Technische Analyse medizinischer Virtual-Reality- Anwendungen}

Für die technische Analyse wurden nach den in Kapitel 2.1. beschriebenen Auswahlkriterien 47 medizinische Virtual-Reality-Anwendungen der Jahre 1996 bis Anfang 1998 ausgewertet. Folgende Aspekte der technischen Realisierung wurden untersucht:

verwendete Steuer- und Kontrollrechner

verwendete Betriebssysteme

verwendete visuelle Anzeigesysteme (und Anzeigeverfahren)

verwendete Eingabesysteme

Die damit gewonnen Daten wurden rein deskriptiv-statistisch ausgewertet, um im Sinne einer Technikrecherche einen Querschnitt der verwendeten Systeme zu erhalten.

Alle Daten wurden nach dem primären, interagierenden Benutzer der VR-Anwendung aufgeschlüsselt (Anwendungsart ,Patient' oder ,Arzt', s.a. Kap. 1.2.4 oder Tab. 1.5., Seite 15). 


\subsubsection{Auswertung}

Tab. 3.1. Häufigkeit der verwendeten Steuer- und Kontrollrechner (Hardware) bei medizinischen $V R$-Anwendungen, aufgeschlüsselt nach Anwendungsart

\begin{tabular}{|c|c|c|c|c|}
\hline & Hardware & $\begin{array}{l}\text { Anwendungsart } \\
\text { Patient als Anwender }\end{array}$ & Arzt als Anwender & Gesamt \\
\hline & High-End-Rechner & 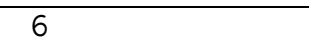 & 9 & 15 \\
\hline & Graphikworkstation & 5 & 11 & 16 \\
\hline & Standard.PC & 8 & 8 & 16 \\
\hline & Keine Angabe & 2 & 0 & 2 \\
\hline Gesamt & & 21 & 28 & 49 \\
\hline
\end{tabular}

* abweichende Gesamtzahl (49) durch Anwendungen, bei denen mehrere Steuerrechner verwendet wurden

In Tabelle 3.1. sind die verwendeten Steuer- und Kontrollrechner (Hardware) von 47 medizinischen VR-Anwendungen aufgeführt, aufgeschlüsselt nach der Anwendungsart. Auffällig ist die gleichmässige Gesamtverteilung auf die drei Hardwareklassen. High-End-Rechner werden bei beiden Anwendungsarten (Arzt bzw. Patient) prozentual gleich häufig eingesetzt, nämlich in jeweils 32\% der Nennungen. Graphikworkstations besitzen einen Anteil von 39\% (11 von 28) bei den Nennungen der ,Arzt'-Anwendungen, aber nur 26\% (5 von 19) bei ,Patient'-Anwendungen. Umgekehrt ist das Verhältnis bei den Standard-Personal Computern: 42\% (8 von 19) der ,Patient'-Anwendungen laufen auf diesen Rechnern, aber lediglich 29 \% (8 von 28) der ,Arzt'·Nennungen.

Tab. 3.2. Häufigkeit der verwendeten Betriebssysteme bei medizinischen VR-Anwendungen, aufgeschlüsselt nach Anwendungsart

\begin{tabular}{lllll}
\hline & Betriebssystem & Anwendungsart & & \\
& & Patient als Anwender & Arzt als Anwender & Gesamt \\
\hline & Unix & 10 & 20 & 30 \\
& Windows & 8 & 7 & 15 \\
& Sonstige & 1 & 0 & 2 \\
& Keine Angabe & 2 & 28 & 2 \\
\hline Gesamt & 21 & 0 & 49 \\
\hline
\end{tabular}

* abweichende Gesamtzahl (49) durch Anwendungen, die sowohl unter Windows als auch Unix lauffähig sind.

In Tabelle 3.2. sind die verwendeten Betriebssysteme der medizinischen VR-Anwendungen aufgeführt. Die Daten zeigen, dass Unix und Windows die dominierenden Betriebssysteme im VR-Bereich sind, wobei Unix mit 61\% (30 von 49) den Hauptteil einnimmt. Bei der Anwendungsart ,Patient‘ werden Unix- und Windows-Systeme etwa gleich häufig eingesetzt, anders ist dies bei der Anwendungsart ,Arzt‘: 71\% (20 von 28) verwenden hier UnixBetriebssysteme. 
Tab. 3.3. Häufigkeit der verwendeten visuellen Anzeigesysteme bei medizinischen VRAnwendungen, aufgeschlüsselt nach Anwendungsart

\begin{tabular}{lllll}
\hline Visuelles Interface & $\begin{array}{l}\text { Anwendungsart } \\
\text { Patient als Anwender }\end{array}$ & Arzt als Anwender & Gesamt \\
\hline Bildschirm & 8 & 15 & 23 \\
Shutter-Brille & 2 & 8 & 10 \\
HMD monoskopisch & 5 & 2 & 7 \\
& HMD stereoskopisch & 1 & 0 & 1 \\
& Einwandprojektion & 1 & 1 & 2 \\
& Mehrwandprojektion & 1 & 0 & 1 \\
& Andere Verfahren & 1 & 0 & 1 \\
& keine Angabe & 2 & 0 & 2 \\
\hline Gesamt & 21 & 26 & \multicolumn{2}{c}{47} \\
\hline
\end{tabular}

In Tabelle 3.3. sind die verwendeten visuellen Anzeigesysteme der medizinischen VRAnwendungen aufgeführt, aufgeschlüsselt nach der Anwendungsart. Die häufigsten Anzeigesysteme sind Bildschirme (23 von $47=49 \%$ ), Shutter-Brillen (10 von $47=21 \%$ ) und monoskopische Head Mounted Displays (7 von 47 = 15\%) mit zusammen 85\% Gesamtanteil (40 von 47). Bildschirme und Shutter-Brillen, die der sogenannten ,Desktop-VR' zugeordnet werden, machen damit $70 \%$ aller Anzeigesysteme aus. Head Mounted Displays und Projektionssysteme, die der Definition von Virtueller Realität am ehesten entsprechen, addieren sich auf lediglich 13\% der Anzeigesysteme. Aufgeschlüsselt nach Anwendungsart verschieben sich die Relationen: bei der Anwendungsart ,Patient' sind $48 \%$ aller Anzeigesysteme der Desktop-VR zuzuordnen (Bildschirm und Shutter-Brille), bei der Anwendungsart ,Arzt' 88\%. Immersive VR-Systeme haben einen Anteil von 38\% bei ,Patient'. Anwendungen, dagegen einen Anteil von nur $12 \%$ bei den Arzt-Anwendungen.

Tab. 3.4. Häufigkeit monoskopischer und stereoskopischer visueller Anzeigesysteme bei medizinischen VR-Anwendungen, aufgeschlüsselt nach Anwendungsart

\begin{tabular}{lllll}
\hline & Anzeigeverfahren & $\begin{array}{l}\text { Anwendungsart } \\
\text { Patient als Anwender }\end{array}$ & Arzt als Anwender & Gesamt \\
& & 16 & 17 & 33 \\
& monoskopisch & 9 & 14 \\
\hline stereoskopisch & 5 & 26 & 47 \\
\hline
\end{tabular}

In Tabelle 3.4. ist der Anteil monoskopischer und stereoskopischer Verfahren bei den verwendeten visuellen Anzeigesystemen aufgeführt. 70\% der Anzeigesysteme benutzen monoskopische Anzeigeverfahren (Bildschirme, HMD monoskopisch), 30\% versorgen den Benutzer mit stereoskopischen Informationen (Bildschirm mit Shutter-Brillen, HMD stereoskopisch, stereoskopische Projektionsverfahren). Innerhalb der Anwendungsarten ist der Anteil der stereoskopischen Verfahren bei ,Arzt'-Anwendungen mit 35\% höher als bei den ,Patient'-Anwendungen (24\%). 
Tab. 3.5. Häufigkeit der verwendeten Eingabesysteme bei medizinischen VR-Anwendungen, aufgeschlüsselt nach Anwendungsart

\begin{tabular}{|c|c|c|c|c|}
\hline & Eingabeinterface & $\begin{array}{l}\text { Anwendungsart } \\
\text { Patient als Anwender }\end{array}$ & Arzt als Anwender & Gesamt \\
\hline & Tastatur & 6 & 10 & 16 \\
\hline & Maus & 2 & 1 & 3 \\
\hline & Joystick & 6 & 1 & 7 \\
\hline & 3D Maus & 1 & 2 & 3 \\
\hline & Tracking-System & 7 & 10 & 17 \\
\hline & Datenhandschuh & 5 & 0 & 5 \\
\hline & individuelles Werkzeug & 2 & 10 & 12 \\
\hline & Sonstiges & 2 & 0 & 2 \\
\hline & Keine Angabe & 1 & 1 & 2 \\
\hline Gesamt & & 32 & 35 & 67 \\
\hline
\end{tabular}

* abweichende Gesamtzahl (67) durch mehrere Eingabesysteme in Anwendungen gleichzeitig verwendete Systeme (z.B. Trackingsystem für die Kopfdrehung, Joystick für weitere Handlungen).

In Tabelle 3.5. sind die verwendeten Eingabesysteme der VR-Anwendungen aufgeführt. $39 \%$ (26 von 67) der verwendeten Eingabesysteme sind VR-untypisch (Tastatur, Joystick, Maus). Den größten Anteil an den Eingabesystemen besitzen Trackingsysteme (25\%). Auffallend ist, dass sich bei der Anwendungsart ,Arzt' die Eingabesysteme zu $86 \%$ auf Tastatur, Tracking. Systeme und individuelle Werkzeuge (z.B. Endoskopiesysteme) verteilen (je 10 von 35). Bei der Anwendungsart ,Patient' sind neben den drei genannten Systemen auch Joysticks und Datenhandschuhe von Bedeutung, wobei individuelle Werkzeuge einen deutlich geringeren Anteil besitzen (2 von 32).

\subsubsection{Zusammenfassung}

Anhand der Literaturanalyse konnten wesentliche technische Komponenten identifiziert werden, die in einem Großteil der untersuchten medizinischen VR-Projekte zum Einsatz kamen. Bei den visuellen Anzeigesystemen waren $70 \%$ handelsübliche Bildschirme, z.T. im Einsatz mit Stereoskopie-fähigen Shutter-Brillen, verwendet worden, das für VR ,charakteristische' Head Mounted Display nur in 15\% der Fälle. Stereoskopische Verfahren machten 30\% der Anwendungen aus. Bei den Eingabesystemen traten im Hinblick auf Anwendungsart ,Arzt” und ,Patient” deutliche Unterschiede zu Tage: bei der ärztlichen Anwendung interagierte dieser fast ausschließlich mit Tastatur, Tracking-Systemen sowie individuell angefertigten Werkzeugen. Für den Patienten als Anwender wurden neben Tastatur und Tracking-Systemen häufig Joystick und Datenhandschuhe verwendet. Bei den Steuer- und Kontrollrechnern fand man eine gleichmäßige Verteilung über die drei Hardware-Klassen HighEnd-Rechner/ Graphikworkstation/ Standard-PC. Im Bereich der Betriebssysteme dominierte Unix deutlich mit $61 \%$ der eingesetzten Systeme. 


\subsection{Organisation und Aufbau des VR-Labors}

\subsubsection{Laborkonzept}

Für den organisatorischen Rahmen des Betriebs und der Evaluation des VR-Labors wurde ein Ablaufplan erstellt. Abbildung 3.1. zeigt ein Organigramm, das Grundlage für Projekte mit klinischen Partnern sein soll.

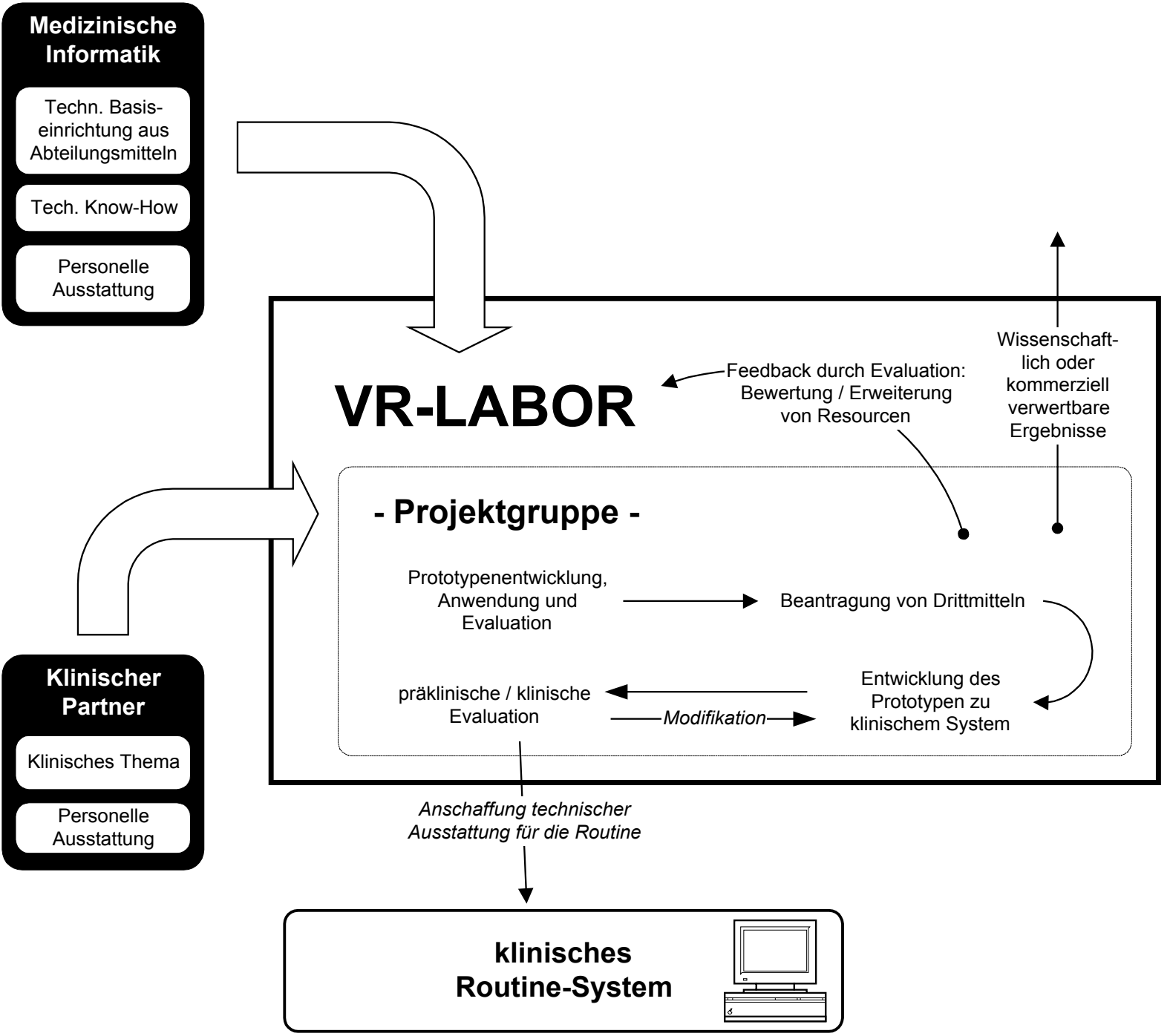

Abb. 3.1. Ablaufdiagramm für die Organisation von Projekten im VR-Labor

Mit Ersteinrichtung des VR-Labors seitens der Abteilung Medizinische Informatik wird eine Basisausstattung - technisch und personell - für interdisziplinäre Projekte zur Verfügung gestellt. Mit klinischen Partnern können Projektgruppen zur Bearbeitung von VR. 
Fragestellungen gebildet werden. Der klinische Partner muss in dieser Phase keine eigenen technischen Ressourcen zur Verfügung stellen, diese werden durch die Basisausstattung abgedeckt. Mit Entwicklung eines Prototypen mit den Kernfunktionen der geplanten VRAnwendung können die Zielvorstellungen präzisiert werden und eine Abschätzung zusätzlich notwendiger personeller und technischer Mittel vorgenommen werden. Diese müssen z.B. über eine Drittmitteleinwerbung finanziert werden. Aufgrund der Erfahrungen mit dem Prototyp kann die Entwicklung einer klinischen VR-Anwendung geplant und durchgeführt werden. Das System wird nun, evtl. über mehrere Evaluationsstufen, zur klinischen Anwendungsreife geführt. Ist die klinische Anwendungsreife erreicht, wird das Projekt organisatorisch weitgehend aus dem VR-Labor gelöst. Erkenntnisse über die Verwendung von VR-Technologien fließen in die Weiterentwicklung des Labor-Konzepts ein.

\subsubsection{Komponenten des eingerichteten VR-Labors}

\subsubsection{ENTWICKLUNGSWERKZEUGE}

Zur Konstruktion der virtuellen Umgebungen und Produktion von Animationen stand das Programm 3D Studio Max zur Verfügung, zur Programmierung von virtuellen Simulationen die dVise-Programmierbibliothek von Division und Microsoft $\mathrm{C}++$. Für Konstruktions- und Demonstrationszwecke wurden Objektbibliotheken angeschafft.

\subsubsection{STEUER- UND KONTROLLSYSTEM}

Die Graphikworkstation Intergraph TDZ 425 (Intergraph Corp., Huntsville, USA) läuft unter dem Windows NT System und besitzt, wie in Tab. 1.2. dargestellt, über ähnlich gute Leistungswerte wie deutlich teurere Unix-Workstations. Angeschafft wurde eine ausgebaute Variante des im OPC-Test angeführten Gerätes (Ausstattung: 2 x Pentium III, 300 Mhz. 2 Graphikkarten mit je 32 MB Graphikspeicher und je 32 MB Texturspeicher, Geometriebeschleuniger. 256 MB Hauptspeicher).

Die Software Dv/reality (Division Ltd., Bristol, UK) dient zur Visualisierung und Steuerung der virtuellen Umgebung. Sie unterstützt die Einbindung sämtlicher kommerzieller VR-Eingabeund Anzeigesysteme.

\subsubsection{EINGABESYSTEME}

Zur Interaktion wurden drei Systeme ausgewählt: ein handelsüblicher Joystick zur Navigation in der Ebene und für einfache Handlungen, das Trackingsystem Polhemus FasTrack (Polhemus Corp., Vermont, USA) mit einem Sensor zur Erfassung von Kopfhaltung oder Bewegung eines Werkzeugs (Leistungsdaten: Abtastrate $120 \mathrm{~Hz}$, Latenzzeit $4 \mathrm{~ms}$, Genauigkeit 0,07 cm / 0,15², Auflösung $0,005 \mathrm{~mm} / 0,025^{\circ}$ ) sowie die Space Mouse (DLR, Oberpfaffenhofen): ein Gerät, mit 
dem man sich durch manuelles Drehen, Kippen sowie Druck und Zug eines Pucks im dreidimensionalen Raum zu Konstruktions- und Navigationszwecken bewegen kann.

\subsubsection{ANZEIGESYSTEME}

Als Komponenten wurden nur visuelle Systeme ausgewählt. Die Wiedergabe von akustischen Reizen erfolgte über die Standard-PC-Soundkarte. Das HMD verfügte zusätzlich über Kopfhörer.

Mit dem Shutter-Brillen-System Stereographics CrystalEyes (Stereographics Corp., San Rafael, USA) konnten auf Kathodenstrahl-Bildschirmen Simulationen stereoskopisch betrachtet werden. Diese Technologie wird zum sogenannten Desktop-VR gerechnet.

Der nicht-stereoskopische Projektor Davis DL450 (ASK/Infocus ASA, Fredrikstad, Norwegen) wurde zur Leinwandprojektion ausgewählt. Dies ermöglichte z.B. eine VR-Anwendung, bei der das Blickfeld des Benutzers durch eine nahe Position vor der Projektionsfläche weitgehend von der virtuellen Realität eingenommen wurde (semi-immersiv). Der Videobeamer konnte auch zu Präsentationszwecken verwendet werden (Auflösung: 800 × 600 Pixel).

Das stereoskopisch oder nicht-stereoskopisch nutzbare Head Mounted Display Virtual Research HMD V8 (Virtual Research Corp., Santa Clara, USA) auf Kathodenstrahlbasis wurde für immersive VR-Anwendungen, vor allem im Zusammenspiel mit dem Trackingsystem für die Aufzeichnung der Kopfbewegung, ausgewählt.

Abb. 3.2. Übersicht über die Komponenten des VR-Labors
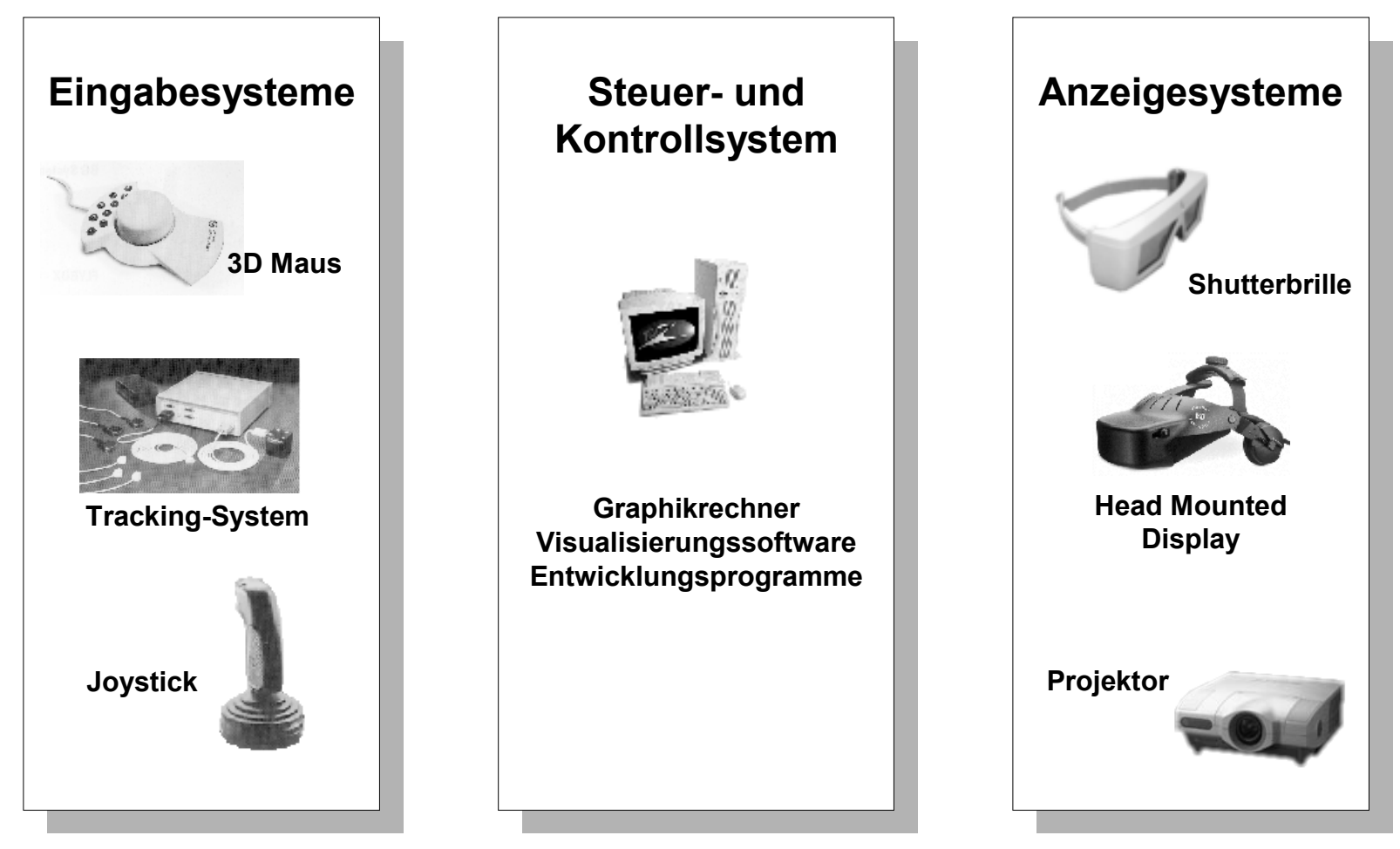


\subsubsection{Zusammenfassung}

Mit der Beschaffung der VR-Komponenten wurde die technische Voraussetzung für die Durchführung von medizinischen VR-Projekten geschaffen. Die Basisausstattung des VR-Labors wurde unter Einbeziehung der Marktübersicht sowie der in Kapitel 3.1. beschriebenen Analyse ausgewählt. Mit Bildschirm (+/-Shutter-Brille), Projektionssystem und Head Mounted Display standen sowohl monoskopische als auch stereoskopische Anzeigeverfahren zur Verfügung. Als VR-spezifisches Eingabesystem wurde ein Trackingsystem beschafft, das in erster Linie in Kombination mit dem HMD zum Head-Tracking verwendet werden sollte. Alternativ hierzu war ein Einsatz des Systems für individuelle Werkzeuge möglich. Als Steuer- und Kontrollrechner wurde eine Workstation auf Basis des Betriebssystems Windows NT ausgewählt, dessen Leistungskennzahlen UNIX-Systemen derselben Preiskategorie nicht nachstanden (Kap. 1.2.2., Tab 1.2.).

Als Grundlage für die Projektarbeit im VR-Labor und das vorgesehene erste interdisziplinäre Projekt wurde ein Laborkonzept erstellt. Organisatorisch wurden damit Projektablauf und Pflichten der beteiligten Gruppen umrissen, inhaltlich wurde der Stellenwert der wissenschaftlichen Evaluation gesichert.

\subsection{Neuropsychologische VR-Anwendung}

Das Neuropsychologische Testsystem wurde gemäß des Entwicklungsentwurfs (Kap. 2.3.3.) anhand von drei Modulen (Virtual-Reality-Testumgebung, Testadministration, Auswertung) implementiert und im Februar 1999 fertiggestellt.

\subsubsection{Virtual-Reality-Testumgebung}

\subsubsection{TECHNISCHE REALISATION}

Als visuelle Anzeigekomponenten konnten ein Computerbildschirm, ein Projektionsgerät oder ein Head Mounted Display verwendet werden. Die Navigation wurde dabei über einen Joystick, die Tastatur oder bei Benutzung des HMDs über ein Tracking-System und die Maus ausgeführt. Zwei Beispiele eingesetzter Konfigurationen sind in den Abb. 3.6. und 3.7. zu sehen.

Das in der Programmiersprache C++ realisierte Modul benötigt Laufzeitbibliotheken des VREntwicklungspakets ,Datapath Realimation‘. Zusammen mit den Bibliotheken kann das Programm auf beliebigen Graphikrechnern mit Windows-Betriebssystemen ohne weitere Zusätze installiert werden. 


\subsubsection{NEUROPSYCHOLOGISCHE TESTUMGEBUNG}

Zwei Aufgabenarten standen für neuropsychologische Testzwecke zur Verfügung: ein naturalistischer Park und ein Labyrinth (Abb. 3.4 u. 3.5.).

Für jede Testaufgabe existierten zwei verschiedene Wegenetze. Allen Wegenetzen war gemeinsam, dass sie 14 Wegepunkte, sechs Kreuzungen, einen Startpunkt, einen Zielpunkt und sechs Sackgassen besaßen (Abb. 3.3.). Vier der Sackgassen befanden sich einen Wegpunkt von der richtigen Route, zwei Sackgassen befanden sich zwei Wegepunkte von der richtigen Route entfernt. Der Park (Abb. 3.4.) wurde mit Objekten und Landschaftsmerkmalen ausgestattet, um Orientierungshilfen zu geben. Wichtige Landschaftsmerkmale waren eine Bergkette, ein Fluss der in einen See mündet, sowie kleinere Hügel innerhalb des Parks. Die kleineren Hügel dienten auch als Sichtschutz, um einzelne Bereiche voneinander abzugrenzen und eine Komplettübersicht über den Park zu verhindern. Als Objekte waren verschieden gestaltete Häuser, Bäume, ein Spielplatz, Zäune,

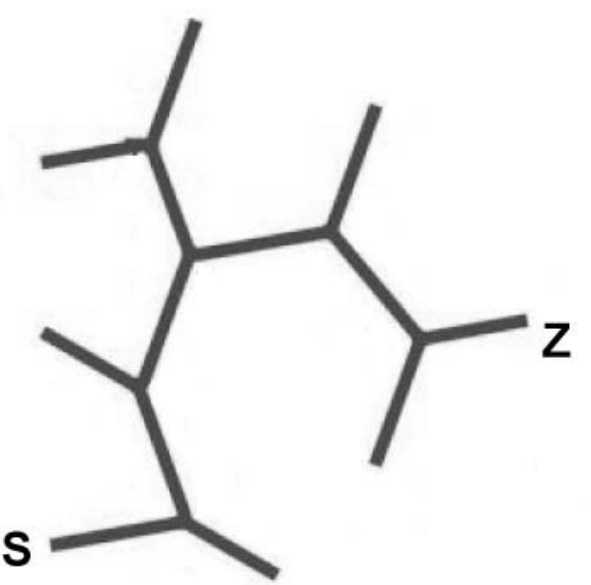

Abb. 3.3. Beispiel eines Wegenetzes $(S=$ Startpunkt, $Z=$ Zielpunkt) Kunstobjekte und Fußgängerbrücken eingebaut.

Wurde der Zielpunkt erreicht, so wurde dies dem Testteilnehmer durch die Einblendung einer Schrifttafel in der virtuellen Umgebung signalisiert. Durch die Objekte war die Geometrie des Parks im Vergleich zum Labyrinth mit 34685 Dreiecksflächen deutlich komplexer.

Das Labyrinth wurde als einfaches Gangsystem gestaltet, bei dem etwa zwei Meter hohe Wände bei einer Kopfhöhe von 1.80 eine Übersicht verhinderten. Das geometrische Modell des Labyrinths enthielt 6345 Dreiecksflächen. Der Startpunkt wurde mit einem roten Kreis markiert, ansonsten existierten für die Testperson keine Objekte als Orientierungshilfen. Abbildung 3.5. gibt einen Eindruck des Labyrinthes aus Sicht der Testperson wieder.

Nach Freigabe des Testdurchgangs durch den Testleiter wurde die Navigation der Testperson aufgezeichnet. Die Navigationsdaten konnten vom Auswertungsmodul zur Berechnung von Leistungsparametern eingelesen werden. Weiterhin war es möglich, mit Hilfe der aufgezeichneten Navigationsdaten den Testdurchgang aus einer Vogelperspektive zu beobachten, bei der die Navigation des Probanden durch einen Pfeil visualisiert war (Wiedergabe-Modus). 

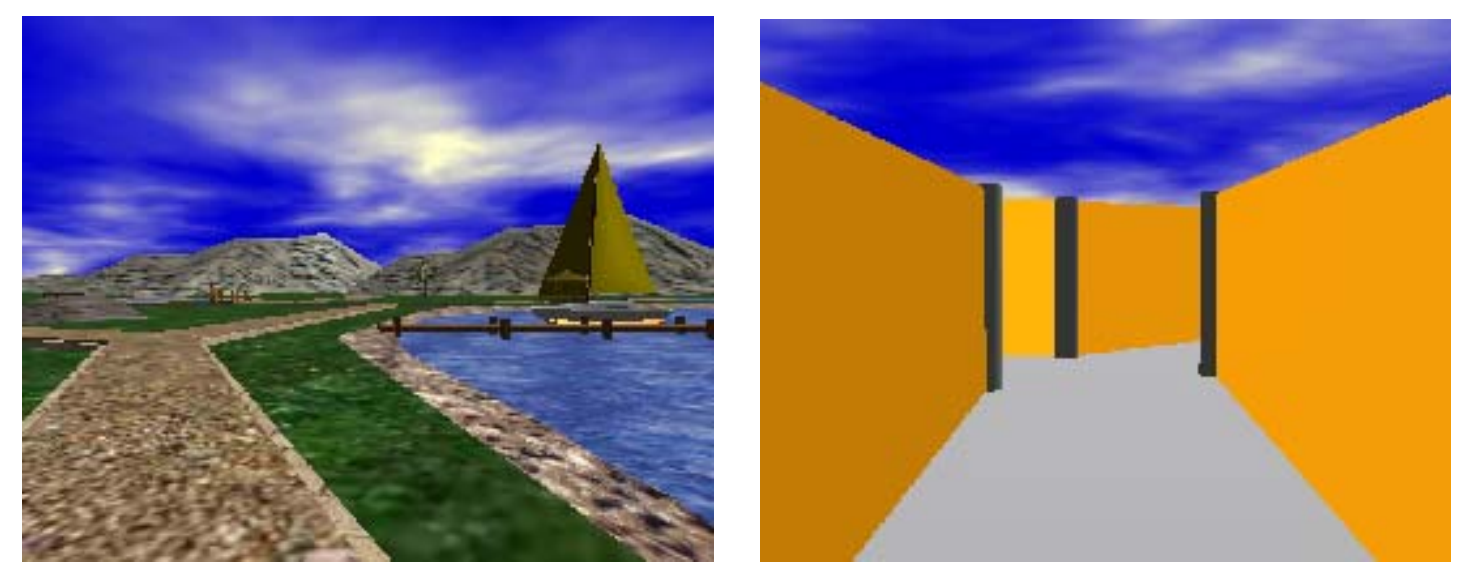

Abb. 3.4. und 3.5. Neuropsychologische VR-Testumgebung: Parkversion (links) und Labyrinthversion (rechts) aus der Perspektive der Testperson
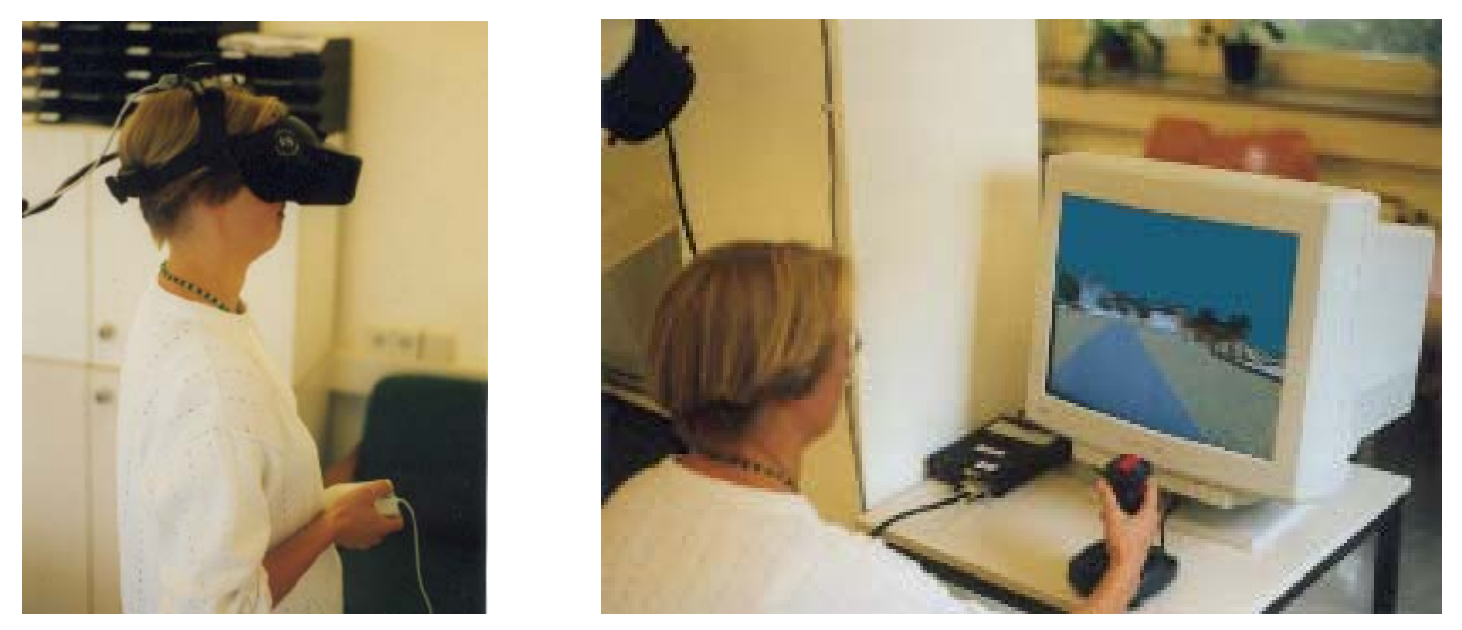

Abb. 3.6. und 3.7. Proband beim VR-Test mit Head Mounted Display/Maus- (links) sowie Bildschirm/Joystick-Kombination (rechts).

\subsubsection{Testadministration}

Für die Testadministration wurde ein Datenbank-basiertes Programm entwickelt. Im Startfenster konnten alle vorhandenen Testpersonen über eine Liste eingesehen werden, für jeden Probanden konnten dabei über ein Formular persönliche Daten erfasst werden (Anhang, Abb. 6.1.). Die Durchführung der Tests wurde mit einem Dialogfenster vereinfacht, mit dem nach Anlage eines Testdurchgangs-Nummer VR-Tests gestartet (Anhang, Abb. 6.3. / 6.5.) und korrespondierende SSQ erfasst (Anhang, Abb. 6.4.) werden konnten. In einem weiteren Dialog war es möglich, eine Liste mit den bereits vorhandenen Testdurchgängen und durchgeführten Tests zu erhalten (Anhang Abb. 6.2.). 


\subsubsection{Auswertung}

Mit dem Auswertungsmodul konnten Navigationsdaten gelesen, Leistungsparameter berechnet sowie diese in eine Datenbank abgelegt werden. Das Programm wurde auf Kommandozeilenebene mit Hilfe der Parameter ,Versuchspersonennummer und ,Testdurchgang، gestartet, die Prozessierung geschah im Weiteren automatisch. Die Ergebnisse wurden unter der Versuchspersonennummer in einer Tabelle in der gemeinsamen Datenbank hinterlegt. Dabei wurden die benötigte Zeit für den Durchgang, das Erreichen des Ziels, die Summe aller Fehler, die Summe aller Entscheidungen, die Summe der mit Entscheidungen verbrachten Zeit, die Summe aller Bewegungen auf korrektem Pfad und die Summe aller Bewegungen auf falschem Pfad aufgezeichnet.

\subsubsection{Zusammenfassung}

Das neuropsychologische Testsystem wurde durch die Abteilung Medizinische Informatik und die Abteilung Psychiatrie/ Schwerpunkt Neuropsychologie der Universität Göttingen entwickelt. Bei der Systementwicklung war durch Umsetzung eines, rapid prototype eine präzisere Abschätzung von Entwicklungsaufwand, inhaltlicher Gestaltung und technisch Machbarem möglich.

Für die präklinische Studie standen als Testumgebungen ein Labyrinth und eine Parklandschaft zum topographisch-räumlichen Lernen zur Verfügung. Für eine vollständige Dokumentation der Testleistung wurde eine kontinuierliche Aufzeichnung der Navigation des Probanden und der begangenen Fehler bei der Testbearbeitung implementiert.

Bei der Entwicklung wurde viel Wert auf die Minimierung nauseogener Faktoren sowie auf eine einfache Handhabung der Testaufgabe gelegt. Die VR-Testumgebung wurde so realisiert, dass eine flexible Anwendung sowohl als Desktop-VR- (Bildschirm, Shutter-Brillen), semi-immersives (Projektion) oder voll-immersives System (Head Mounted Display) möglich war. Als Eingabeschnittstellen wurden Tastatur, Maus, Joystick und Tracking-System angebunden.

Zur komfortablen Testadministration wurde eine datenbankorientierte Anwendung entwickelt. Eine Auswertung von Testleistungen war hiermit quantitativ (Fehler, Zeit) und visuell (Wiedergabemodus der Testdurchführung) möglich. 


\subsection{Präklinische Evaluation des neuropsychologischen Testsystems}

Die präklinische Evaluation des Testsystems wurde im März 1999 durchgeführt. Im Rahmen dieser Arbeit werden Ergebnisse zur Benutzerevaluation und zum Auftreten unerwünschter Nebenwirkungen vorgestellt.

\subsubsection{Messzeitpunkte und statistische Methoden}

Jeder Proband durchlief den in Kap. 2.4.3. dargestellten Versuchsablauf. Die soziodemographischen Daten wurden vor der Testung erhoben. Die Nebenwirkungen wurden mit dem Simulator Sickness Questionnaire (SSQ) an fünf Zeitpunkten dokumentiert während der insgesamt 12 vorgesehenen Testdurchgänge. Drei der SSQ wurden zur Auswertung herangezogen:

- to: SSQ vor Beginn der Testung, noch keine VR-Exposition erfolgt

- t1: SSQ am Ende des Projektionsmodus nach Durchführung von 6-Testdurchgängen

- t2: SSQ nach Abschluss des HMD-Modus, in der Regel nach dem zwölften Durchgang oder bei Testabbruch.

Die Einzelscores des Simulator Sickness Questionnaire (Total-Score, Nausea-Score, Oculomotor-Score und Disorientation-Score) wurden zunächst berechnet (siehe Anhang 6.3.) und anhand einer einfaktoriellen Varianzanalyse mit einem dreistufigen Messwiederholungsmodell untersucht (Faktor: Messzeitpunkt). Als zweiseitiges Signifikanzniveau wurde $\alpha=0.05$ gewählt. Da die Probanden in der Regel beide Anzeigemodalitäten sequentiell durchliefen, konnte damit ein orientierender Vergleich zwischen Projektion und HMD erfolgen. Hierzu wurden jeweils einfaktorielle Varianzanalysen mit einem Messwiederholungsmodell durchgeführt. Weitere Faktoren, die Einfluss auf das Auftreten von Simulator Sickness haben konnten, wurden in dieser Analyse u.a. wegen der geringen Stichprobengröße nicht untersucht. Die deskriptive Statisitk der zugrunde liegenden SSQ-Scores ist in Tab. 3.8. dargestellt.

Die Benutzerbewertung des Testsystems wurde nach Durchführung der VR-Testaufgaben durchgeführt. Hierbei wurden neben deskriptiv-statistischen Auswertungen $t$-Tests durchgeführt ( $\alpha=0.05$ ), um Bewertungen der beiden Anzeigemodalitäten zu vergleichen.

\subsubsection{Beschreibung der Stichprobe}

Es wurden insgesamt 22 Probanden getestet, hiervon waren 12 weiblich und 10 männlich. Das Durchschnittsalter lag bei 29.5 Jahren (Minimum 22, Maximum 50, Standardabweichung 6.83), den größten Anteil besaßen hierbei die 20-29jährigen mit 73\%, lediglich zwei 
Probanden waren älter als 40. Keiner der Probanden litt an einer psychiatrischen oder neurologischen Erkrankung. Alle Probanden hatten mindestens einen Gymnasialabschluss, 14 der 22 Probanden waren Studenten, 8 arbeiteten in einem Angestelltenverhältnis oder waren freiberuflich tätig. Auf einer 6-stufigen Likert-Skala siedelten $23 \%$ der Probanden ihre Computerkenntnisse im oberen Drittel und $69 \%$ im mittleren Drittel an, der Mittelwert lag bei 3.14 (1 = sehr gut, 6 = gering; Standardabweichung 1.25).

\subsubsection{Benutzerevaluation: Technische Realisation des Testsystems}

Nach Durchführung der Virtual-Reality-Tests wurden die Probanden mit einem Fragebogen (POST-Test) zur technischen Umsetzung hinsichtlich Realitätsgrad, Interaktion und Präferenzen befragt.

Tab. 3.6. Bewertungen der virtuellen Umgebung unter Berücksichtigung der verwendeten Anzeigemodalität

\begin{tabular}{lll}
\hline $\begin{array}{l}\text { Bewertung } \\
(1=\text { sehr hoch bis } 6=\text { sehr niedrig) }\end{array}$ & HMD & Projektion \\
\hline Realitätsgrad virtuelle Umgebung & $3.38(1.60)$ & MW (SD) \\
Realitätsgrad Umhersehen ${ }^{A}$ & $2.43(1.36)$ & $3.86(1.62)$ \\
Grad des ,Hineinversetztseins' (Immersion) $^{\text {B }}$ & $2.71(1.49)$ & $3.48(1.21)$ \\
\hline
\end{tabular}

Legende (MW = Mittelwert, SD = Standardabweichung):

A $t$-Test bei gepaarten Stichproben: HMD realistischer als Projektion $(p<0.05)$

B $t$-Test bei gepaarten Stichproben: HMD immersiver als Projektion $(p<0.05)$

Korrelation n. Pearson: Projektion ( $\mathrm{p}$ je < 0.01): Immersion*Realitäsgrad 0.71, Immersion*Umhersehen 0.56 HMD (p je < 0.01): Immersion*Realitäsgrad 0.82, Immersion*Umhersehen 0.76

Ein Proband führte die Testaufgaben nur im Projektionsmodus durch, da das HMD auf seine Kopfgröße nicht angepasst werden konnte.

Die Handhabung der VR-Eingabesysteme (Anhang 6.5., Frage 2.1-2.4) gestaltete sich für alle Probanden nach eigener Einschätzung problemlos: sowohl im Projektionsmodus mit Navigation durch die Tastatur als auch bei Benutzung des HMD mit Tracking-System und Maus als Eingabeschnittstelle verneinten alle Probanden Schwierigkeiten mit der Bedienung.

11 von 21 Probanden empfanden das HMD als beklemmend (Anhang 6.5., Frage 7). Nach der Benutzung beider Anzeigeverfahren entschieden sich bei der Frage nach einer Präferenz (Anhang 6.5., Frage 8) 11 Testpersonen für das Projektionssystem, 9 für das HMD (kein signifikanter Unterschied im $\chi^{2}$-Test).

Den Realitätsgrad der virtuellen Umgebung (1=hoch, 6=gering; Anhang 6.5., Frage 3.1-3.2.) ordneten die meisten Probanden sowohl das HMD als auch den Projektionsmodus in der unteren Hälfte der Skala ein. Im $t$-Test bei gepaarten Stichproben ergab sich kein signifikanter 
intraindividueller Unterschied der abhängigen Variablen Realitätsgrad/HMD und Realitätsgrad/Projektion (Tab. 3.6).

Beim Umhersehen in der virtuellen Umgebung wurde das HMD mit seinem Tracking-System im Vergleich zum Projektionsmodus mit Tastatur als realistischer beurteilt (Anhang 6.5., Frage 4.1.-4.2; Tab. 3.6.). Die Prüfung auf eine Signifikanz mittels $t$-Test bei gepaarten Stichproben (abhängige Variablen: Umhersehen/HMD, Umhersehen/Projektion) fiel positiv aus $(p<0.05)$.

Der subjektive Immersionsgrad (Anhang 6.5., Frage 5.1.5.2.) wurde ebenfalls mit dem HMD durchschnittlich höher eingeschätzt (Tab. 3.6.) als mit dem Projektionssystem, beim t-Test bei gepaarten Stichproben (abhängige Variablen: Immersion/HMD, Immersion/Projektion) ergab dies einen signifikanten Unterschied $(p<0.05)$

Die weiterführende Analyse der Bewertungen (Realitätsgrad der virtuellen Umgebung, Realitätsgrad des Umhersehens, Immersion) zeigte, dass diese auf signifikantem Niveau miteinander korrelierten (Korrelation n. Pearson; $p<0.01$; s.a. Tab. 3.6.).

Anhand von Beobachtungen während der Testdurchführung, freien Anmerkungen der Probanden und der Nachbesprechung konnten essentielle Probleme bei der Verwendung des Testsystems festgehalten werden. Nahezu alle Probanden kritisierten den Tragekomfort des HMD: vor allem das Gewicht, dessen Schwerpunkt auf Nasenrücken und Stirn drückt, wurde bemängelt. Bei zwei Probanden konnte das HMD wegen Kopfgröße/Haaren nicht angepasst werden. Ein Proband bestand darauf, den Test mit Festhalten des HMD durchzuführen, der andere Proband führte den Test nur im Projektionsmodus durch. Positiv war anzumerken, dass sich bei der Durchführung der Trainingsdurchgänge alle Probanden innerhalb der vorgesehenen fünf Minuten mit der Handhabung des Systems zurechtfanden.

\subsubsection{Ergebnisse zu unerwünschten Nebenwirkungen}

\subsubsection{TESTABBRÜCHE AUFGRUND VON NEBENWIRKUNGEN}

Zu allen Ergebnissen hinsichtlich unerwünschter Nebenwirkungen in der präklinischen Studie sei noch einmal auf eine mögliche Konfundierung von Messzeitpunkt und Anzeigemodus durch den Testablauf hingewiesen: grundsätzlich wurde zunächst die Testung im Projektionsmodus, anschließend im HMD-Modus durchgeführt (s.a. Kap. 2.4.3.3.).

Bei korrekter Durchführung des Tests brach ein Proband den Test vor Durchführung aller Durchgänge ab, dies entspricht einer Quote von 4.76 \% (1 von 21 im HMD-Modus).

Anzumerken ist, dass es bei einer zweiten Testung zu einem Testabbruch kam: hier wurde zum ersten Mal für den Projektionsmodus ein Projektor mit größerer Leuchtstärke benutzt; der leistungstärkere Lüfter des Gerätes führte zunächst unbemerkt zur Beeinträchtigung der Signalqualität des HMD-Tracking-Systems. Bei den Testdurchgängen mit HMD trat bei 
bestimmten räumlichen Konfigurationen (Bewegung der Probandin zum Projektor hin) eine Instabilität des Bildes auf. Nach mehrmaligem Auftreten dieses Effektes wies die Probandin darauf hin, dass ihr unwohl sei. Nach Auffinden und Beseitigung der Fehlerquelle führte die Probandin den Versuch zunächst weiter, brach aber bei Durchgang Nr. 10 die Testung endgültig ab. Da der Abbruch vermutlich durch die technische Fehlfunktion induziert oder zumindest beeinflusst wurde, fand 6 Wochen später eine erneute Testung der Probandin unter HMD-Bedingung statt. Diese nacherhobenen Werte sind in den nachfolgenden Statistiken statt der ersterhobenen Werte verwendet worden. Nur für die Auswertung der SSQ-Scores bei Testabbruch sind die ursprünglichen Werte verwendet worden, diese sind in Tabelle 3.8. aufgeführt. Die Probanden, die den Test aufgrund Unwohlseins abbrechen mussten, wurden hinsichtlich der Dauer der Symptome befragt; beide Probanden gaben an, dass nach ca. 1-11/2 Stunden die Symptome vollständig verschwunden waren.

\subsubsection{SIMULATOR-SICKNESS-SCORES IM TESTVERLAUF}

Im Gesamtverlauf über alle 3 Messzeitpunkte (einfaktorielle Varianzanalyse mit dreistufigem Messwiederholungsmodell, Faktor Messzeitpunkt to/t1/t2; $n=21$ ) ergaben sich für den Total-, Nausea-, Oculomotor- und Disorientation-Score keine signifikanten Veränderungen. Im folgenden wurden daher die beiden Testabschnitte einzeln untersucht (Projektionsmodus to/t1, HMD-Modus t1/t2).

Tab. 3.7. Scores der erhobenen Simulator Sickness Questionnaires

\begin{tabular}{lccc}
\hline SSQ Skala & t0 $(\mathbf{n}=\mathbf{2 2})$ & $\mathbf{t 1}(\mathbf{n}=\mathbf{2 2})$ & $\mathbf{t 2}(\mathbf{n}=\mathbf{2 1})$ \\
& MW $(\mathrm{SD})$ & MW $(\mathrm{SD})$ & MW $(\mathrm{SD})$ \\
\hline Nausea-Score & $9.1(9.5)$ & $11.3(12.4)$ & $15.9(21.2)$ \\
Oculomotor-Score & $15.2(16.5)$ & $12.4(15.6)^{\mathrm{A}}$ & $13.0(13.2)$ \\
Disorientation-Score & $1.2(2.4)$ & $2.4(3.9)$ & $3.9(5.5)^{\mathrm{B}}$ \\
Total-Score & $12.2(12.5)$ & $12.9(14.3)$ & $16.6(18.5)$ \\
\hline
\end{tabular}

Legende $(\mathrm{MW}=$ Mittelwert, SD = Standardabweichung):

* Ein Proband führte die Testaufgaben mit HMD nicht durch, da das HMD auf die Kopfgröße nicht angepasst werden konnte.

A einfaktorielle Varianzanalyse mit zweistufigem Messwiederholungsmodell, Faktor Messzeitpunkt t0/t1: signifikanter Abfall des Oculomotor-Score $(p<0.05)$.

$B$ einfaktorielle Varianzanalyse mit zweistufigem Messwiederholungsmodell, Faktor Messzeitpunkt $t 1 / t 2$ : signifikanter Anstieg des Disorientation-Score $(p<0.05)$.

Nach Durchführung der Testdurchgänge im Projektionsmodus ließen sich für die SSQ-Scores ebenfalls keine Anstiege nachweisen (einfaktorielle Varianzanalyse mit zweistufigem Messwiederholungsmodell, Faktor Messzeitpunkt to/t1), auffällig war jedoch ein signifikanter Abfall des Oculomotor-Scores $[F(1,21)=6.721 ; p<0.05 ; n=22]$ (s.a. Tab. 3.7.).

In den darauf folgenden Testdurchgängen mit Verwendung des $\underline{H M D}$ zeigte sich ein signifikanter Anstieg des Disorientation-Score $[F(1,20)=5,455, p<0.05 ; n=21]$ bei der 
einfaktoriellen Varianzanalyse mit zweistufigem Messwiederholungsmodell (Faktor: Messzeitpunkt t1/t2). Für die übrigen Scores zeigten sich keine Auffälligkeiten.

In Tabelle 3.8. sind die SSQ.Scores im Testverlauf klassifiziert nach regulärem Testende versus Abbruch des Testes wegen Nebenwirkungen klassifiziert. Eine interferenzstatistische Analyse der Daten hinsichtlich eines möglichen Gruppenunterschiede ist aufgrund der kleinen Fallzahlen hier nicht möglich.

In dieser Auswertung fällt auf, dass bereits zu Testbeginn die Scores der Abbrechergruppe im Mittel deutlich über denen der regulären Gruppe lagen. Während des Projektionsmodus stiegen Nausea- und Disorientation-Score in der Abbrechergruppe leicht an, der Total-Score blieb stabil und der Oculomotor-Score fiel ab. Überproportional stiegen die SSQ-Scores der Abbrechergruppe im HMD-Modus, für den Total-Score war dies das 2.3fache des Ausgangswertes. Der Total-Score der regulären Gruppe stieg in deutlich geringerem Maße an (1.4fach). Anhand der Mittelwerte in Tabelle 3.8. lässt sich demnach nachvollziehen, dass die Abbrechergruppe einen deutlicheren Anstieg im Verlauf des HMD-Modus zeigte als die Probanden mit regulärem Ende. Dieser Unterschied ließ sich allerdings nicht für den Projektionsmodus nachweisen.

Tab. 3.8. SSQ Skalen klassifiziert nach Abbruch wegen Nebenwirkungen ( $n=2)$ versus reguläres Ende der Testung ( $n=19)$ *

\begin{tabular}{llccl}
\hline SSQ Skala & Beendigung & t0 & t1 & t2 \\
& & MW (SD) & MW (SD) & MW (SD) \\
\hline Nausea-Score & Regulär & $7.6(8.8)$ & $8.5(11.0)$ & $11.5(14.1)$ \\
& Abbruch & $19.1(13.5)$ & $28.6(0.0)$ & $76.3(13.5)$ \\
\hline Oculomotor-Score & Regulär & $11.6(12.7)$ & $9.6(11.8)$ & $11.0(12.5)$ \\
& Abbruch & $26.6(16.1)$ & $15.2(10.7)$ & $30.2(10.7)$ \\
\hline Disorientation-Score & Regulär & $2.9(7.5)$ & $5.1(9.5)$ & $11.7(19.3)$ \\
& Abbruch & $13.9(19.7)$ & $20.9(9.8)$ & $41.8(0.0)$ \\
\hline Total-Score & Regulär & $9.4(9.6)$ & $9.4(10.3)$ & $13.6(15.6)$ \\
& Abbruch & $24.3(18.5)$ & $24.3(2.6)$ & $56.1(10.6)$ \\
\hline
\end{tabular}

Legende (MW = Mittelwert, SD = Standardabweichung):

* für die Auswertung der SSQ-Scores bei Testabbruch sind die ersterhobenen Werte des Probanden verwendet worden, bei dem eine technische Fehlfunktion die Symptome induzierte (s. Kap. 3.4.4.1.)

\subsubsection{Zusammenfassung}

In der präklinischen Studie wurden 22 gesunde Probanden getestet. Zunächst wurden die Testaufgaben in der Konfiguration ,Projektionsmodus/ Tastatur' durchgeführt, anschließend in gleicher Sitzung mit ,HMD/Head-Tracking'-Konfiguration bearbeitet.

Bei der Benutzerevaluation gaben die Probanden keine Schwierigkeiten bei der Bedienung des Systems an. Über die Hälfte der Testteilnehmer empfand das HMD als beklemmend, der schlechte Tragekomfort dieses Systems wurde auch in der offenen Nachbefragung 
beanstandet. Hinsichtlich der Beurteilung des Realismus der Testumgebung konnte kein Effekt der Anzeigemodalität (HMD vs. Projektion) ausgemacht werden. Das HMD wurde allerdings signifikant besser bewertet hinsichtlich eines realitätsnahen Umhersehens und der Immersion. Die weiterführende Auswertung zeigte eine Verknüpfung von Realismus und Immersion im Sinne einer signifikanten Korrelation.

Bei den Ergebnissen zu unerwünschten Nebenwirkungen wurde eine Abbruchquote von $4.8 \%$ verzeichnet. Bei der Analyse der Simulator Sickness Questionnaires zeigte sich, dass über den Gesamtverlauf (1. Projektionsmodus, 2. HMD-Modus) der Testung keine signifikanten Änderungen der SSQ-Scores eintraten. In der weiteren Auswertung der Testabschnitte konnte jedoch im Projektionsmodus ein signifikanter Abfall des Oculomotor-Scores sowie im HMD. Modus ein signifikanter Anstieg des Disorientation-Scores nachgewiesen werden. In einer deskriptiven Analyse zeigt sich des Weiteren, dass die Gruppe der Testabbrecher vor allem im HMD-Modus gegenüber der Gruppe mit regulärer Beendigung des Tests deutlichere SSQ. Score-Anstiege hatte. Bei allen Ergebnissen muss berücksichtigt werden, dass durch den Versuchsaufbau (zunächst immer Projektion, danach HMD) eine Konfundierung von Testzeitpunkt und Anzeigemodus möglich ist.

\subsubsection{Veränderungen am Testsystem als Konsequenz der präklinischen Evaluation}

Aufgrund der Evaluationsergebnisse der präklinischen Studie wurden Modifikationen am Testsystem vorgenommen. Bei den Anzeigesystemen wurde das Head Mounted Display vom Typ 'Virtual Research V8' (2 kg Gewicht) durch ein 'Sony Glasstron' ersetzt, das mit $150 \mathrm{~g}$ wesentlich leichter und komfortabler war (Abb. 3.8.). Leider handelte es sich auch hierbei nicht um ein kabelloses System, so dass eine von einer Deckenkonstruktion unabhängige Bewegung des Probanden weiterhin nicht möglich war. Neben dem Austausch des HMD wurden kleinere Veränderungen zur Minimierung der Simulator-Sickness-Symptome vorgenommen: als Anregung von Benutzern wurden weniger kräftige und irritierende Farben - vor allem bei der Labyrinthversion - verwendet (Abb. 3.10.). Die Gänge wurden deutlich breiter gestaltet (vgl. Abb. 3.5. und 3.10.). Aus testpsychologischen Überlegungen wurden kritische Punkte der neuropsychologischen Operationalisierung aufgegriffen. Der Park wurde hinsichtlich der Komplexität des Wegenetzes erweitert und das Labyrinth vereinfacht. Park und Labyrinth wurden im Erscheinungsbild weiterentwickelt (z.B. breitere Wege, zusätzliche Landmarken, realistischere Gestaltung; s. Abb. 3.11./3.12.). Die Aufgabenstellung für die Testpersonen wurde aus Verständnisgründen modifiziert: in der präklinischen Studie sollte das Wegenetz ungezielt zum Auffinden des Zielpunktes exploriert werden, in der klinischen Studie wurden Tontöpfe am Ende eines jeden Ganges aufgestellt, die auf einen Inhalt überprüft werden sollten. Als neue Testversion wurden Aufgaben zum exekutiven Lernen hinzugefügt, bei der Regeln erkannt und angewandt werden sollten (Abb. 3.9.). 
Abb. 3.8.

Sony Glasstron Head Mounted Display
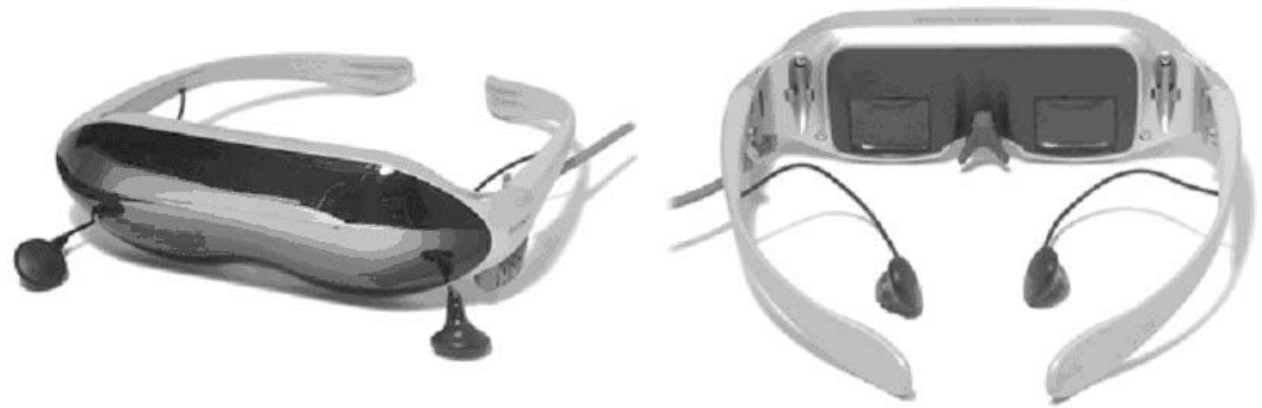

Abb. 3.9./3.10. Vogelperspektive aus der Exekutivversion (links), Probandensicht in der Labyrinthaufgabe (rechts)
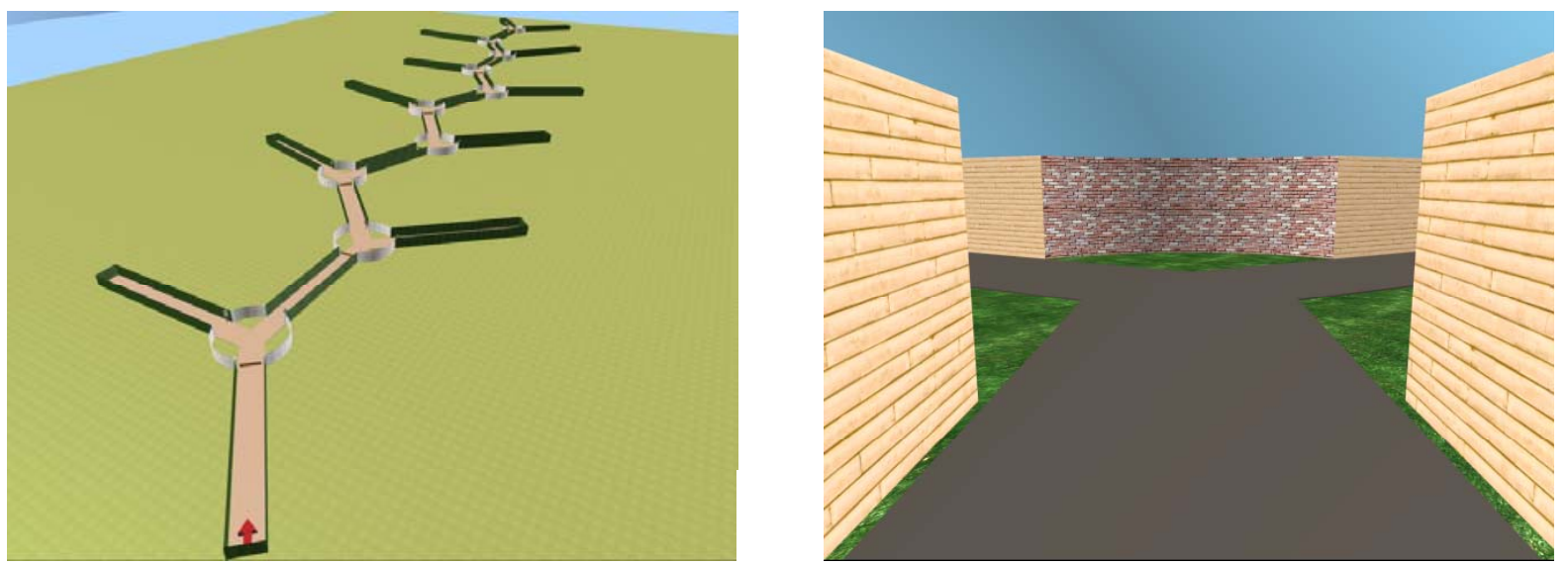

Abb. 3.11./3.12. Park aus Benutzer-(links) und Vogelperspektive (rechts)
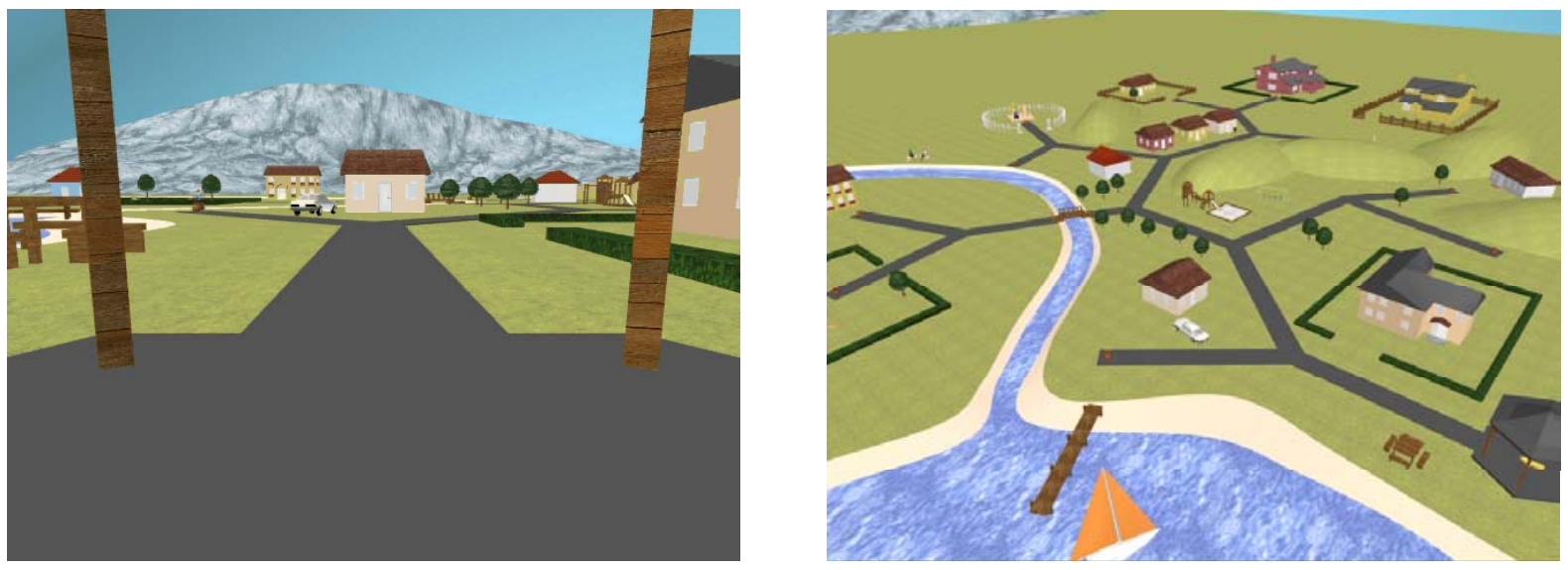


\subsection{Klinische Evaluation des Testsystems}

Die klinische Evaluation des Testsystems wurde von August 1999 bis August 2002 durchgeführt. Dabei durchliefen die Teilnehmer den in Kap. 2.4.4. dargestellten Versuchsablauf. Im Rahmen dieser Arbeit werden Ergebnisse zur Benutzerevaluation und zum Auftreten unerwünschter Nebenwirkungen vorgestellt.

\subsubsection{Beschreibung der Stichprobe der klinischen Studie}

215 Teilnehmer, 87 weibliche und 128 männliche, führten mindestens eine VR-Aufgabe durch und wurden so in die klinische Studie aufgenommen. Das mittlere Alter betrug 47.8 Jahre (Standardabweichung 15.2, Minimum 15, Maximum 76). Neben 75 gesunden Probanden der Kontrollgruppe (CTRL) wurden 40 Patienten vor einem epilepsiechirurgischen Eingriff (EPI. PRÄ), 25 Patienten nach einem epilepsiechirurgischen Eingriff (EPI-POST), 27 Schlaganfallpatienten (STROKE) und 48 Patienten mit V.a. Alzheimer-Demenz (AD) eingeschlossen. Merkmale der einzelnen Gruppen sind Tab. 3.9. aufgeschlüsselt.

Tab. 3. 9. Geschlecht, Alter und Computervorkenntnisse der Teilnehmergruppen

\begin{tabular}{|c|c|c|c|c|c|c|}
\hline & \multicolumn{6}{|c|}{ Teilnehmergruppe } \\
\hline & CTRL & EPI-PRÄ & EPI-POST & STROKE & $A D$ & Gesamt \\
\hline$\overline{\text { Anzahl }(w / m)^{A}}$ & $75(30 / 45)$ & $40(18 / 22)$ & $25(14 / 11)$ & $27(11 / 16)$ & $48(14 / 34)$ & $215(87 / 128)$ \\
\hline Alter $(\mathrm{MW} / \mathrm{SD})^{\mathbf{B}}$ & $45.2(17.1)$ & $37.2(12.1)$ & $38.9(11.3)$ & $60.0(8.6)$ & $58.4(8.0)$ & $47.8(15.2)$ \\
\hline $\begin{array}{l}\text { Computervorkenntnisse } \\
\text { (MW/SD) C, D }\end{array}$ & $1.14(0.69)$ & $0.97(0.79)$ & $0.96(0.86)$ & $0.81(0.74)$ & $0.70(0.78)$ & $0.95(0.77)$ \\
\hline
\end{tabular}

Legende (MW = Mittelwert, SD = Standardabweichung):

${ }^{A} \mathrm{w}=$ weiblich, $\mathrm{m}=$ männlich

B post-hoc Bonferonni-Mehrfachvergleiche: AD / STROKE signifikant älter als CTRL / EPI-POST / EPI-PRÄ (je p<0.01), CTRL signifikant älter als EPI.PRÄ $(p<0.05)$

c Antwortskala: 0 = keine Kenntnisse, 1 = vereinzelte Kenntnisse, 2 = gute Kenntnisse

D post-hoc Bonferonni-Mehrfachvergleiche: CTRL signifikant besser Vorkenntnisse als AD $(p<0.05)$.

Bei der Untersuchung der Teilnehmergruppen hinsichtlich der Geschlechterzusammensetzung ergaben sich bei der statistischen Auswertung mittels $\chi^{2}$-Test keine signifikanten Unterschiede. Die einfaktorielle Varianzanalyse hinsichtlich der Teilnehmergruppe als Zwischensubjektfaktor zeigte signifikante Unterschiede für die abhängigen Variablen ,Alter' $[F(4,199)=18.17, p<0.01$; $n=215]$ sowie ,Computervorkenntnisse' $[F(4,193)=2.44, p<0.05 ; n=209]$.

Die post-hoc durchgeführten Mehrfachvergleiche mit Adjustierung nach Bonferonni $(p<0.05)$ zeigten, dass die AD. und STROKE-Teilnehmer signifikant älter waren als die CTRL-, EPI-PRÄund EPI-POST-Teilnehmer (jeweils $p<0.01$ ) sowie die CTRL-Gruppe älter war als die EPI-PRÄ. Gruppe war $(p=0.026)$. STROKE und AD unterschieden sich hinsichtlich des Alters nicht 
signifikant voneinander. Die post-hoc Bonferonni-Testung der Computervorkenntnisse bezüglich der Teilnehmergruppen zeigte lediglich einen signifikanten Unterschied zwischen der Gruppe AD und CTRL $(p<0.05)$, die Kontrollgruppe besaß damit signifikant bessere Computerkenntnisse. Die Computervorkenntnisse korrelierten auf signifikantem Niveau negativ mit dem Alter, somit sanken erwartungsgemäß mit steigendem Alter die Kenntnisse der Computerbedienung (Korrelation n. Pearson -0.39, $\mathrm{p}<0.01 ; \mathrm{n}=209$ ).

\subsubsection{Benutzerbewertung und Praktikabilität}

Die 215 Teilnehmer wurden nach Durchführung der Virtual-Reality-Aufgaben hinsichtlich der Benutzerbewertung und Praktikabilität des Testsystems befragt (Fragebogen s. Anhang 6.6.). Die Verteilung der Testteilnehmer auf die bei der Testung verwendeten Anzeigemodalitäten (HMD, Monitor, Projektion) ist in Tabelle 3.10. angegeben. Da keine Randomisierung auf die verschiedenen Anzeigemodalitäten vorgenommen wurde und älteren Probanden eher zu den nicht-immersiven Verfahren (Monitor, Projektion) geraten wurde, zeigten sich entsprechend diesem Bias bezüglich der Verteilung der Teilnehmergruppen auf die Anzeigemodalitäten im $\chi^{2}$-Test signifikante Unterschiede $(p<0.01 ; s$. Tab. 3.10.). Als eigene Entität unter den verwendeten Anzeigemodalitäten bei den Testteilnehmern waren es 17 Probanden, die während der maximal drei VR-Testaufgaben die Anzeigemodalität wechselten. Dies geschah unter anderem wegen technischer Probleme (4 Fälle), Unwohlsein (3 Fälle) oder aus Komfortgründen (2). 6 Probanden aus der Gruppe der HMD gaben bei der Nachbefragung eine generelle Unbehaglichkeit bei der Verwendung dieses Gerätes an (Anhang 6.6., Frage 7).

Tab.3.10. Verwendete Anzeigesysteme bei der Testung, aufgeschlüsselt nach Teilnehmergruppen

\begin{tabular}{llllll}
\hline \multicolumn{7}{l}{ Anzeigemodalität } & & & & Gesamt \\
Teilnehmergruppe & HMD & Monitor & Projektion & Wechsler & 75 \\
\hline CTRL & 41 & 18 & 10 & 6 & 40 \\
EPI.PRÄ & 18 & 17 & 3 & 2 & 25 \\
EPI.POST & 15 & 5 & 0 & 5 & 27 \\
STROKE & 0 & 22 & 1 & 0 & 48 \\
AD & 4 & 31 & 13 & $17^{\text {B }}$ & 215 \\
\hline Gesamt & 78 & 93 & 27 & & \\
\hline
\end{tabular}

Legende:

A $\chi^{2}$-Test: signifikante Unterschiede in der erwarteten Verteilung der Anzeigemodalitäten auf die Gruppen $(p<0.01)$

$\mathbf{B}$ von 17 Teilnehmern wechselten 9 in den ersten beiden Durchgängen

Die Gruppe der Teilnehmer, die einen Wechsel der Anzeigemodalität vornahm, wurde statistisch auf Unterschiede zu den Teilnehmern untersucht, die die Modalität beibehielten. In der einfaktoriellen Varianzanalyse zeigte sich bezüglich des Alters (abhängige Variable) zwischen den beiden Gruppen (gewechselt/ nicht gewechselt) kein signifikanter Unterschied. Die Verteilung von Anzeigemodalität, Geschlecht und Computervorkenntnissen differierte im $\chi^{2}$. 
Test nicht signifikant. Die Teilnehmerzusammensetzung der Wechslergruppe zeigte im $\chi^{2}$-Test signifikante Auffälligkeiten $(p<0.05)$ : aus der Gruppe AD wechselte niemand, aus der Gruppe EPI-POST (5 Wechsler) und STROKE (4 Wechsler) mehr Teilnehmer als erwartet.

In 10 von 215 Fällen gaben Teilnehmer in der Nachbefragung an, Probleme mit der Gerätebedienung gehabt zu haben (Anhang 6.6., Frage 6). Signifikante Auffälligkeiten bei der statistischen Untersuchung in der Gruppe der Teilnehmer mit Gerätebedienungsproblemen im Vergleich mit Probanden ohne Bedienungsprobleme ergaben sicht nicht. Hierzu wurde hinsichtlich des Altersunterschiedes der beiden Gruppen eine einfaktorielle Varianzanalyse durchgeführt (abhängige Variable: Alter; Faktor: Gruppe Bedienungsprobleme/ keine Bedienungsprobleme), wobei kein signifikanter Unterschied bestand. Im $\chi^{2}$-Test unterschieden sich die beiden Gruppen ebenfalls nicht signifikant voneinander für die Variablen Geschlecht, Computervorkenntnisse, Gruppe und Anzeigemodus.

Von 215 Teilnehmern brachen 7 während oder nach der ersten VR-Testaufgabe ab. 208 begannen die zweite Testaufgabe und 171 die dritte, die ausschließlich in der Exekutiv-Version bestand (Tab. 3.11.). Die niedrige Teilnehmerzahl in der dritten Aufgabe (171 vs. 215 bei der ersten Aufgabe) erklärt sich dadurch, dass diese optional durchgeführt wurde, wenn dies die zur Verfügung stehende Zeit und die subjektive Konzentrationsspanne des Probanden zuließen.

Tab. 3.11. Verteilung der Probanden während des Testverlaufs auf die Testaufgaben

\begin{tabular}{llcl}
\hline Testaufgabe & $\begin{array}{l}\text { Probanden } \\
\text { in Test Nr. 1 }\end{array}$ & $\begin{array}{c}\text { Probanden } \\
\text { In Test. Nr. 2 }\end{array}$ & $\begin{array}{l}\text { Probanden } \\
\text { In Test. Nr. 3. }\end{array}$ \\
\hline Park & 120 & 93 & $\cdot$ \\
Labyrinth & 95 & 113 & $\cdot$ \\
Executive & - & 2 & 171 \\
Fehlend & - & 7 & 44 \\
\hline Gesamt & 215 & 215 & 215 \\
\hline
\end{tabular}

Eine nicht-reguläre Beendigung des Tests bei der Aufgabenbearbeitung wurde in insgesamt 17 Fällen verzeichnet (Tab. 3.12.). Häufigste Ursache dafür waren Misserfolg oder Überforderung bei der Aufgabenbearbeitung (7 Personen), Nebenwirkungen waren dreimal die Ursache des Testabbruchs. In den übrigen Fällen wurden Zeitmangel, technische Probleme oder keine Gründe angegeben. Auf die Abbrecher aufgrund von Nebenwirkungen wird im Kapitel 3.5.4. noch näher eingegangen.

Tab. 3.12. Gründe für Nichtfortsetzen des Tests

\begin{tabular}{llll}
\hline Grund & $\begin{array}{l}\text { Während oder nach } \\
\text { Aufgabe Nr. 1 }\end{array}$ & $\begin{array}{l}\text { Während oder nach } \\
\text { Aufgabe Nr. 2 }\end{array}$ & $\begin{array}{l}\text { Während } \\
\text { Aufgabe Nr. 3. }\end{array}$ \\
\hline Misserfolg/ Überforderung & 4 & 3 & $\cdot$ \\
Simulator Sickness & - & 3 & - \\
Sonstiges & 3 & 4 & - \\
Reguläres Ende & - & 35 & - \\
\hline Gesamt & 7 & 44 & 0 \\
\hline
\end{tabular}




\subsubsection{Bewertung von Immersion und Realismus}

Die Bewertung der Immersion und des Realismus durch die Teilnehmer in der Nachbefragung wurde auf Unterschiede zwischen den einzelnen Aufgabenarten (Park, Labyrinth, Exekutiv) sowie auf den Einfluss der verwendeten Anzeigemodalität untersucht.

Jeder Teilnehmer bewertete Immersion und Realismus jeweils für jede der maximal drei Aufgabenarten, die er durchlief (Park, Labyrinth, Exekutiv). Zur Auswertung gelangten daher sechs Variablen: Immersion/Park, Immersion/Labyrinth, Immersion/Exekutiv, Realismus/Park, Realismus/Labyrinth und Realismus/Exekutiv (Anhang 6.6., Fragen 9/10).

Die Bewertung des Realismus wurde auf Unterschiede zwischen den Aufgabenarten sowie auf den Einfluss der verwendeten Anzeigemodalität untersucht.

Die Antworten der Testpersonen zum Realismus der Testumgebung wurden mit einer einfaktoriellen Varianzanalyse untersucht (ANOVA; Zwischensubjektfaktor Aufgabenart: Park, Labyrinth, Exekutiv). Hierbei fand sich ein signifikanter Unterschied zwischen den Gruppen $[F(2,553)=5.597, p<0.01 ; 556$ Antworten]. In der post-hoc Bonferonni-Testung zeigte sich eine signifikant bessere Beurteilung des Realismus der Aufgabenart ,Park' im Vergleich zum Labyrinth $(p<0.05)$, die Vergleiche von Park/Exekutiv und Labyrinth/Exekutiv blieben ohne signifikantes Ergebnis (Tab. 3.13.).

Zur Untersuchung eines möglichen Einflusses der Anzeigemodalität auf die Bewertung des Realismus wurde zunächst aus den maximal drei Einzelbewertungen (Realismus/Park, Realismus/Labyrinth und Realismus/Exekutiv) pro Testperson ein Durchschnittswert gebildet (Realismus/Gesamt). Dieser wurde mittels einer einfaktoriellen Varianzanalyse statistisch untersucht (ANOVA; Zwischensubjektfaktor Anzeigemodalität: HMD, Projektion, Monitor). Signifikante Unterschiede in der durchschnittlichen Bewertung des Realismus zwischen den Anzeigemodalitäten kamen nicht zu Tage.

In gleicher Art wurde die Testpersonenbewertung der Immersion auf Einflüsse der Aufgabenart und der Anzeigemodalität untersucht.

In der einfaktoriellen Varianzanalyse (ANOVA; Zwischensubjektfaktor Aufgabenart: Park, Labyrinth, Exekutiv) zeigte sich ein deutlicher Einfluss der Aufgabenart auf die Bewertung der Immersion $[\mathrm{F}(2,564)=15.407, \mathrm{p}<0.01 ; 566$ Antworten]. Anhand der post-hoc BonferonniTestung ließ sich eine signifikant bessere Immersion im Park gegenüber dem Labyrinth und der Exekutivaufgabe ablesen (je $\mathrm{p}<0.01$ ), signifikante Unterschiede zwischen Labyrinth und Exekutivaufgabe bestanden jedoch nicht.

Ein Einfluss der Anzeigemodalität auf die Bewertung der Immersion wurde mittels einfaktorieller Varianzanalyse untersucht. Hierzu wurde wiederum aus den drei Einzelbewertungen (Immersion/Park, Immersion/Labyrinth, Immersion/Exekutiv) der 
Durchschnittswert ,Immersion/Gesamt' für jede Testperson gebildet. In der Varianzanalyse (ANOVA; Zwischensubjektfaktor Anzeigemodalität: HMD, Projektion, Monitor) zeigte sich kein Einfluss der Anzeigemodalität auf die Bewertung der Immersion.

Tab.3.13. Bewertung von Realismus und Immersion aufgeschlüsselt nach verwendeter Aufgabenart

\begin{tabular}{lll}
\hline Aufgabenart & $\begin{array}{l}\text { Bewertung }^{\text {A }} \\
\text { Realismus MW (SD) }\end{array}$ & Immersion MW (SD) \\
\hline Park & $3.3(1.3)^{\mathrm{B}}$ & $3.5(1.2)^{\mathrm{C}}$ \\
Labyrinth & $2.9(1.3)$ & $2.8(1.3)$ \\
Exekutiv & $3.1(1.3)$ & $3.0(1.3)$ \\
\hline Gesamt & $3.1(1.3)$ & $3.1(1.3)$ \\
\hline
\end{tabular}

Legende (MW = Mittelwert, SD = Standardabweichung):

A 1 = niedrig, 5 = hoch

B ANOVA / post-hoc Bonferonni-Testung: Realimus Aufgabenart Park signifikant höher bewertet als Labyrinth $(p<0.05)$

c ANOVA / post-hoc Bonferonni-Testung: Immersion Aufgabenart Park signifikant höher bewertet als Labyrinth und Exekutiv (je $p<0.01$ )

Tab. 3.14. Gesamtbewertung von Realismus und Immersion aufgeschlüsselt nach verwendeter Anzeigemodalität

\begin{tabular}{lll}
\hline Aufgabenart & $\begin{array}{l}\text { Bewertung } \\
\text { Realismus MW (SD) }\end{array}$ & Immersion MW (SD) \\
\hline HMD & $3.1(1.1)$ & $3.1(1.0)$ \\
Monitor & $3.0(1.2)$ & $3.1(1.1)$ \\
Projektion & $2.8(1.1)$ & $2.9(1.2)$ \\
\hline Gesamt & $3.0(1.2)$ & $3.1(1.1)$ \\
\hline
\end{tabular}

Legende (MW = Mittelwert, SD = Standardabweichung):

A $1=$ niedrig, $5=$ hoch

Inwiefern ein Zusammenhang zwischen der Bewertung der Immersion und des Realismus besteht, wurde mittels einer Korrelationsberechnung untersucht. Hierbei zeigte sich, dass die Gesamtbewertung von Immersion und Realismus signifikant miteinander korrelieren (Signifikanz n. Pearson=0.437; $p<0.01 ; n=195$ ).

Beobachtungen aus der Testdurchführung und Anmerkungen der Testteilnehmer der klinischen Studie Als limitierender Faktor hinsichtlich einer ungestörten Interaktion bei der Benutzung des HMD wurde vor allem das Strom-/Datenkabel des Head Mounted Display genannt. Das Gewicht und das Design des alternativ eingesetzten HMD (Sony Glasstron) führten dazu, dass das die Anpassung an die Kopfgröße (Brillendesign) und Gewicht unproblematisch waren. Ältere Probanden zeigten eine deutliche Zurückhaltung und Unsicherheit bei der Benutzung des HMD (s.a. 2.4.4.3.). 


\subsubsection{Auswertung der erfassten Nebenwirkungen}

\subsubsection{MESSZEITPUNKTE UND STATISTISCHE METHODEN}

Bei der Auswertung der Simulator-Sickness-Daten (Nausea-, Oculomotor-, Disorientation und Total-Score) wurden den Analysen folgende Messzeitpunkte zugrunde gelegt:

- Messzeitpunkt to: vor der ersten VR-Testaufgabe (prä).

- Messzeitpunkt t1: nach der ersten VR-Testaufgabe (post-1).

- Messzeitpunkt t2: nach der zweiten VR-Testaufgabe (post-2).

- $\quad$ Messzeitpunkt t3: nach der dritten (optionalen) VR-Testaufgabe (post-3).

Das zweiseitige Signifikanzniveau in allen statistischen Auswertungen (Varianzanalysen, Korrelationen) wurde mit $\alpha=0.05$ festgesetzt. Zur Analyse der Simulator-Sickness-Daten auf beeinflussende Faktoren wurden lediglich vollständige Daten der ersten beiden Testaufgaben verwendet (to- t2). Ausgeschlossen wurden Daten der Teilnehmer, die in diesen Durchgängen die Anzeigemodalität wechselten, die Exekutiv-Aufgabe bearbeiteten, nur die erste Aufgabe bearbeiteten oder deren SSQ-Bögen nicht vollständig waren. Dies erschien sinnvoll und gerechtfertigt aus folgenden Gründen:

Alle Abbrecher aufgrund von Nebenwirkungen waren in diesem Auswertungsmodell inbegriffen (Tab. 3.12.).

- Die erste und zweite Aufgabe (Park und Labyrinth) wurden in der Reihenfolge stratifiziert (siehe Tab. 3.11.), während die dritte Aufgabe immer in der Exekutiv-Version bestand. Ein Einschluss der Daten der dritten Aufgabe hätte eine statistische Untersuchung auf einen Einfluss des Testinhalts (Aufgabenart) und der Testaufgabenreihenfolge erschwert.

- Der dritte Durchgang wurde nur von 171 der 215 Teilnehmer durchgeführt, sodass hinsichtlich der Auswertung die Datenbasis nochmals geringer ausgefallen wäre.

Von initial 215 Teilnehmern wurden somit 194 Teilnehmer in die weiterführende Auswertung der Simulator-Sickness-Daten aufgenommen. Von den Teilnehmern führten 83 die Tests mit HMD, 87 mit Monitor und 24 mit Projektion durch.

\subsubsection{GRUNDAUSWERTUNG}

Die reguläre Testdurchführung brachen drei von 215 Teilnehmern wegen Nebenwirkungen ab, dies entspricht einer Gesamtquote von 1.4 \% (s. a. Tab. 3.12.). Da sich alle drei Abbrecher aus der HMD-Gruppe $(n=78)$ rekrutierten, bestand für diese Modalität eine Abbruchquote von 3.8 \%. Zwei Abbrecher stammten aus der CTRL-Gruppe, einer aus der Gruppe EPI-PRÄ. Die beobachtete Verteilung der Abbrecher in den Gruppen (Anzeigemodalität, Teilnehmer) wich im $\chi^{2}$-Test nicht signifikant von der erwarteten Verteilung ab. Die erfassten SSQ-Scores der drei 
Teilnehmer zum Zeitpunkt des Testabbruchs sind in Tabelle 3.15. aufgeführt. Kein Teilnehmer aus der Wechsel-, Monitor- oder Projektionsgruppe brach den Test wegen Nebenwirkungen ab. In Tabelle 3.15. ist in einer Übersicht die deskriptive Statistik für die SSQ-Scores über die Gesamtheit der Stichprobe angegeben, da in den nachfolgenden interferenzstatistischen Untersuchungen auf eine beschränkte Stichprobe zurückgegriffen wurde (s. Kap. 3.5.4.1.).

Tab. 3.15. Scores der erhobenen Simulator Sickness Questionnaires

\begin{tabular}{lcccr}
\hline SSQ-Score & $\mathbf{t 0}(\mathrm{n}=215)$ & $\mathbf{t 1}(\mathrm{n}=215)$ & $\mathbf{t 2}(\mathrm{n}=207)^{\mathbf{A}}$ & $\mathbf{t 3}(\mathrm{n}=171)$ \\
MW (SD) & & & & \\
\hline Nausea-Score & $7.9(12.0)$ & $8.7(13.8)$ & $8.5(17.4)$ & $7.3(12.5)$ \\
Oculomotor-Score & $11.0(14.0)$ & $11.1(13.9)$ & $10.9(14.9)$ & $10.0(14.2)$ \\
Disorientation-Score & $2.3(4.5)$ & $2.9(5.7)$ & $3.0(5.8)$ & $2.8(5.5)$ \\
Total-Score & $10.8(14.3)$ & $11.8(15.6)$ & $11.7(17.3)$ & $10.6(15.2)$ \\
\hline
\end{tabular}

Legende (MW = Mittelwert, SD = Standardabweichung):

A einer der 208 nachbefragten Teilnehmer füllte keinen Fragebogen nach Durchführung der zweiten Aufgabe aus.

\subsubsection{INTERFERENZSTATISTISCHE UNTERSUCHUNG DER SIMULATOR-SICKNESS- SCORES}

\subsubsection{1. Überprüfung des Einflusses des Alters auf SSQ-Scores}

Vor Durchführung der Varianzanalysen wurde die Notwendigkeit der Berücksichtigung des Alters als Kovariate überprüft. Die SSQ-Scores zu den einzelnen Messzeitpunkten wurden mit dem Alter korreliert. Hier zeigte sich lediglich bei einer von 12 Berechnungen ein signifikantes Ergebnis, und zwar für die Nausea-Score bei t1 (Korrelation n. Pearson -0.153, p<0.05; $\mathrm{n}=194)$. Im zweiten Schritt wurden die Differenzen zwischen den Messzeitpunkten ( 11 - to, $t 2$. to, t2 - t1) mit dem Alter korreliert, wobei keine signifikanten Korrelationen aufgezeigt werden konnten. Dennoch wurden zur weiteren Absicherung alle folgenden Varianzanalysen mit und ohne die Kovariate Alter berechnet. Einmalig wurde durch Berücksichtigung der Kovariate ein marginal signifikantes Ergebnis zu einem nicht signifikanten. Da sich jedoch keine Differenzen hinsichtlich der signifikanten Ergebnisse ergaben, werden im Folgenden nur die Varianzanalysen ohne die Kovariate Alter dargestellt.

\subsection{Einfluss des Messzeitpunktes auf die SSQ-Scores}

Zur Auswertung der SSQ-Scores wurden im ersten Schritt einfaktorielle Varianzanalysen mit einem dreistufigen Messwiederholungsmodell durchgeführt.

Zunächst wurden einfaktorielle Varianzanalysen mit dreistufigem Messwiederholungsmodell (abhängige Variablen: SSQ Total-, Nausea-, Oculomotor- und Disorientation-Score; Faktor: Messzeitpunkt to, t1, t2) durchgeführt. Im Gesamtverlauf ergab sich für den Total-Score hierbei kein signifikanter Anstieg oder Abfall, ebenso für die Unterscores ,Nausea' und ,Oculomotor'. Der Disorientation-Score stieg jedoch signifikant im Gesamtverlauf an (s. Tab. 3.16.) 
$[F(2,386)=3.153, p<0.05 ; n=194]$. In der post-hoc Analyse zur Untersuchung der einzelnen Testabschnitte (to/t1, t1/t2) zeigte sich in der einfaktoriellen Varianzanalyse mit zweistufigem Messwiederholungsmodell, dass sich die signifikante Veränderung von Disorientation-Score und Messzeitpunkt auf den ersten Testabschnitt (to/t1) beschränkte $[F(1,193)=3.691, p=0.056$; $\mathrm{n}=194$; marginal signifikant].

Tab. 3.16. Scores der erhobenen Simulator Sickness Questionnaires ( $n=194)$

\begin{tabular}{lccc}
\hline SSQ Score & t0 & t1 & t2 \\
MW (SD) & & $7.8(13.1)$ & $8.3(17.6)$ \\
\hline Nausea-Score & $7.1(11.2)$ & $10.3(13.3)$ & $10.1(13.9)$ \\
Oculomotor-Score & $10.3(13.9)$ & $2.6(5.2)$ & $2.7(5.5)$ \\
Disorientation-Score A B & $2.1(4.3)$ & $10.8(14.5)$ & $11.0(16.6)$ \\
Total-Score & $10.0(13.8)$ & & \\
\hline
\end{tabular}

Legende (MW = Mittelwert, $\mathrm{SD}=$ Standardabweichung): einfaktorielle Varianzanalyse mit

A dreistufigem Messwiederholungsmodell: Disorientation-Score steigt im Gesamtverlauf (t0-t2) signifikant an $(p<0.05)$

B zweistufigem Messwiederholungsmodell: Disorientation-Score steigt im Verlauf zwischen t0 und t1 marginal signifikant an $(p=0.056)$

In einer nachfolgenden Untersuchung wurde die Gruppe der Abbrecher aufgrund von Nebenwirkungen besonders betrachtet. Diese wurden mit denjenigen Testpersonen verglichen, die die Testung regulär oder aus sonstigen Gründen (s.a. Tab 3.12.) beendeten. Aufgrund der geringen Gruppengröße wurde lediglich eine deskriptive Analyse durchgeführt. An der Tabelle 3.17. lässt sich ablesen, dass ab dem Messzeitpunkt $t 1$ die SSQ-Mittelwerte der Abbrecher deutlich über denen der anderen Testpersonen lagen. Während für die Gruppe der regulären Beendigung der maximale Faktor für einen SSQ-Score-Anstieg 1.2 betrug (to vs. t2, Disorientation-Score), zeigte sich ein bis zu siebenfacher Anstieg der Werte bei den Abbrechern (Nausea-Score).

Tab. 3.17. SSQ-Skalen klassifiziert nach Abbruch wegen Nebenwirkungen $(n=3)$ versus reguläres oder sonstiges Ende der Testung ( $n=191)$

\begin{tabular}{llccc}
\hline SSQ Skala & Beendigung & t0 & t1 & t2 \\
& & MW (SD) & MW (SD) & \multicolumn{1}{c}{ MW (SD) } \\
\hline Nausea-Score & Regulär / sonstige & $7.0(11.0)$ & $7.2(12.1)$ & $7.0(12.1)$ \\
& Abbruch & $12.7(14.6)$ & $44.5(22.0)$ & $89.0(77.7)$ \\
\hline Oculomotor-Score & Regulär / sonstige & $10.4(13.9)$ & $10.1(13.3)$ & $9.7(13.1)$ \\
& Abbruch & $7.6(7.6)$ & $22.7(13.1)$ & $37.9(33.0)$ \\
\hline Disorientation-Score & Regulär / sonstige & $2.1(4.3)$ & $2.6(5.2)$ & $2.6(5.3)$ \\
& Abbruch & $1.2(2.2)$ & $3.7(3.7)$ & $12.5(7.8)$ \\
\hline Total-Score & Regulär / sonstige & $10.0(13.9)$ & $10.4(14.3)$ & $10.1(14.1)$ \\
& Abbruch & $10.0(11.4)$ & $32.4(16.9)$ & $66.1(54.1)$ \\
\hline
\end{tabular}

Legende: $(M W=$ Mittelwert, $\mathrm{SD}=$ Standardabweichung $)$ 


\subsubsection{3. Überprüfung möglicher Einflussfaktoren auf die SSQ-Scores}

Im zweiten Schritt wurden mögliche Einflussfaktoren auf die SSQ-Scores untersucht. Dies waren im Einzelnen:

- die verwendete Anzeigemodalität (HMD, Monitor, Projektionssystem)

- das Geschlecht (weiblich, männlich)

- die Zugehörigkeit zu einer Teilnehmergruppe (CTRL, EPI-PRÄ, EPI-POST, STROKE, AD)

- die Reihenfolge der Aufgabenbearbeitung (Labyrinth/Park vs. Park/Labyrinth).

Hierzu wurden zweifaktorielle Varianzanalysen mit dreistufigem Messwiederholungsmodell durchgeführt (abhängige Variablen: SSQ Total-, Nausea-, Oculomotor- und DisorientationScore; Faktor: möglicher Einflussfaktor + Messzeitpunkt).

Die ANZEIGEMODALITÄT (HMD, Monitor, Projektionssystem) zeigte einen signifikanten Einfluss auf den Total-Score in der Varianzanalyse $[F(4,364)=3.229, p<0.05 ; n=194]$ (Mittelwerte s. Tab. 3.18.). Im nächsten Schritt wurden daraufhin post-hoc Einzelvergleiche zwischen den drei Anzeigemodalitäten durchgeführt (HMD/Monitor, HMD/Projektion, Projektion/Monitor), wiederum in einem dreistufigen Messwiederholungsmodell.

Tab. 3.18. Deskriptive Statistik für die SSQ-Scores zu den drei Messzeitpunkten, unterteilt nach Anzeigemodalitäten

\begin{tabular}{|c|c|c|c|c|}
\hline $\begin{array}{l}\text { SSQ-Score } \\
\text { MW (SD) }\end{array}$ & Art der Anzeige & to & t1 & t2 \\
\hline \multirow[t]{3}{*}{ Nausea-Score ${ }^{\mathbf{A}}$} & HMD & $6.9(11.1)$ & $9.4(16.2)$ & $10.8(23.9)$ \\
\hline & Monitor & $7.0(11.5)$ & $6.7(10.6)$ & $6.7(11.0)$ \\
\hline & Projektion & $8.3(10.6)$ & $6.0(7.9)$ & $5.2(8.4)$ \\
\hline \multirow[t]{3}{*}{ Oculomotor-Score } & HMD & $9.6(11.2)$ & $10.8(12.9)$ & $11.1(14.2)$ \\
\hline & Monitor & $10.4(13.7)$ & $9.8(13.3)$ & $9.1(12.8)$ \\
\hline & Projektion & $12.6(21.2)$ & $10.4(15.5)$ & $10.1(16.7)$ \\
\hline \multirow[t]{3}{*}{ Disorientation-Score $^{\mathbf{B}}$} & HMD & $1.4(2.6)$ & $2.9(6.0)$ & $3.3(6.1)$ \\
\hline & Monitor & $2.1(4.3)$ & $2.2(4.3)$ & $2.0(4.1)$ \\
\hline & Projektion & $4.2(7.6)$ & $3.3(5.4)$ & $3.4(7.4)$ \\
\hline \multirow[t]{3}{*}{ Total-Score $^{\mathbf{C}}$} & HMD & $8.9(10.8)$ & $11.9(16.2)$ & $13.0(19.9)$ \\
\hline & Monitor & $9.9(14.0)$ & $9.6(13.0)$ & $9.1(12.6)$ \\
\hline & Projektion & $13.7(20.7)$ & $10.7(13.7)$ & $10.4(16.5)$ \\
\hline
\end{tabular}

Legende (MW = Mittelwert, SD = Standardabweichung):

zweifaktorielle Varianzanalyse mit dreistufigem Messwiederholungsmodell: Anzeigemodalität und Messzeitpunkt interagieren signifikant für:

A Nausea-Score $(p<0.05)$

post-hoc Einzelvergleich: HMD > Monitor $(p=0.06)$ und HMD > Projektion $(p=0.08)$, marginal signifikant

B Disorientation-Score $(p<0.05)$

post-hoc-Einzelvergleich: HMD > Monitor $(p<0.01)$ und HMD > Projektion $(p<0.05)$

c Total-Score $(p<0.05)$

post-hoc-Einzelvergleich: HMD > Monitor $(p<0.01)$ und HMD > Projektion $(p<0.05)$ 
Das HMD zeigte in den durchgeführten Einzelvergleichen einen signifikant stärkeren Einfluss auf den Anstieg des Total-Scores als der Monitor $[F(2,336)=5.028, p<0.01 ; n=170]$ und die Projektion $[F(2,210)=3.705, p<0.05 ; n=111]$. Zwischen Monitor und Projektion ergab sich kein signifikanter Unterschied im Einfluss auf den Total-Score.

Nach Untersuchung des Total-Score erfolgte die Analyse der Subskalen. Für die abhängige Variable Nausea-Score konnte zum Zeitpunkt to, t1 und t2 in der zweifaktoriellen Varianzanalyse mit dreistufigem Messwiederholungsmodell ein signifikanter Einfluss der Anzeigemodalität nachgewiesen werden $[F(4,382)=2.597, p<0.05 ; n=194]$. In der post-hoc-Analyse via zweifaktorieller Varianzanalyse im dreistufigen Messwiederholungsmodell zeigten sich in den Einzelvergleichen zwischen den Anzeigemodalitäten lediglich marginal signifikante Ergebnisse. Hierbei führte wiederum das HMD zu einem Anstieg der Nausea-Scores (HMD vs. Monitor: $F(2,236)=2.837, p=0.06 ; n=170 ; \quad H M D$ vs. Projektion: $F(2,210)=2.520, p=0.083 ; n=107$; Monitor vs. Projektion: nicht signifikant).

Bei der statistischen Untersuchung des Oculomotor-Scores zeigte sich kein signifikanter Einfluss der Anzeigemodalität für einen Anstieg oder Abfall des Scores.

Bei der Untersuchung des Disorientation-Score ließen sich ähnliche Ergebnisse wie beim Total-Score beobachten. Die Anzeigemodalität hatte einen signifikanten Einfluss auf den Score $[F(4,364)=3.281, p<0.05 ; n=194]$. Die drei post-hoc durchgeführten Einzelvergleiche mit jeweils zwei Anzeigemodalitäten umfassenden Stichproben zeigten, dass das HMD im Vergleich zu Monitor $[\mathrm{F}(2,336)=6.359, \mathrm{p}<0.01 ; \mathrm{n}=170]$ und Projektion $[F(2,210)=3.782, p<0.05 ; n=111]$ zu einem signifikanten Anstieg bei dem Disorientation-Score führtw. Zwischen Monitor und Projektion existierte kein signifikanter Unterschied.

In einer weiteren post-hoc-Analyse wurde untersucht, inwieweit sich der nachgewiesene Einfluss der Anzeigemodalität auf die SSQ Scores auf einzelne Testabschnitte beschränkte (Aufgabe 1 entspricht to vs. t1, Aufgabe 2 entspricht t1 vs. t2). Hierzu wurden jeweils zweifaktorielle Varianzanalysen in einem zweistufigen Messwiederholungsmodell für die Messzeitpunkte to vs. $t 1$ und t1 vs. t2 durchgeführt (abhängige Variablen: SSQ Total-, Nausea-, Oculomotor- und Disorientation-Score; Faktor: Anzeigemodalität+ Messzeitpunkt).

Die zweifaktorielle Varianzanalyse für to gegen t1 (Aufgabe 1) zeigte einen signifikanten Einfluss der Anzeigemodalität auf den Total-Score $[F(2,191)=4.864, p<0.01 ; n=194]$, Nausea-Score $[F(2,191)=3.611, p<0.05 ; n=194]$ und Disorientation-Score $[F(2,191)=4.561, p<0.05 ; n=194]$. Für den Oculomotor-Score ließ sich kein Einfluss nachweisen. In der Analyse von t1 gegen t2 (entspricht Aufgabe 2) wurden keine signifikanten Einflüsse der Anzeigemodalität auf die SSQ. Scores aufgezeigt, womit in Zusammenschau letztere Ergebnisse mit dem dreistufigen Messwiederholungsmodell korrespondieren. Somit beruhen die nachgewiesenen signifikanten Veränderungen im Gesamtverlauf auf den Veränderungen zwischen Messzeitpunkt to und $t 1$. 
In der zweifaktoriellen Varianzanalyse mit dreistufigem Messwiederholungsmodell zeigte sich keine Interaktion von GESCHLECHT und Messzeitpunkt auf den Total-Score im Sinne eines signifikanten Anstiegs oder Abfalls im Testverlauf. Allerdings zeigte sich ein von den Messzeitpunkten unabhängiger, konstanter signifikanter Einfluss der Geschlechtszugehörigkeit auf den Total-Score $[F(1,182)=5.108, p<0.05 ; n=194]$. Die post-hoc Auswertung mittels dreier $t$-Tests bei unabhängigen Stichproben ergab (abhängige Variablen: Total-Score jeweils zum Zeitpunkt t1, t2 und t3; kategorisiert nach Geschlecht), dass für alle Messzeitpunkte der TotalScore der Frauen signifikant über dem der Männer lag (Tab. 3.19.). Für den abhängige Variable Nausea-Score zum Zeitpunkt to, t1 und t2 ergaben sich keine signifikanten Einflüsse in den zweifaktoriellen Varianzanalysen mit dreistufigem Messwiederholungsmodell für die Faktoren Geschlecht. Bei der statistischen Untersuchung des Oculomotor-Scores zeigte sich ebenfalls keine signifikante Interaktion zwischen Geschlecht und Messwiederholungsfaktor. Für das Geschlecht wurde jedoch wiederum beobachtet, dass dieses einen vom Testverlauf unabhängigen Einfluss ausübt $[F(1,182)=6.833, p<0.05 ; n=194]$ : die Werte der Frauen lagen signifikant über denen der Männer (Tab. 3.19.). Bei der Untersuchung des DisorientationScore ließen sich ähnliche Ergebnisse wie beim Total- und Oculomotor-Score beobachten. Das Geschlecht zeigte keinen Interaktionseffekt mit dem Messzeitpunkt, jedoch lagen die Werte des Disorientation-Scores bei den Frauen bei zwei von drei Messzeitpunkten ( $t 1$ und $t 2$ ) signifikant über denen der Männer (Tab. 3.19.) $[F(1,182)=4.051, p<0.05 ; n=194]$.

Tab. 3.19. Deskriptive Statistik für die SSQ-Scores zu den drei Messzeitpunkten, aufgeschlüsselt nach Geschlecht

\begin{tabular}{|c|c|c|c|c|}
\hline $\begin{array}{l}\text { SSQ Score } \\
M W(S D)\end{array}$ & & to & t1 & t2 \\
\hline \multirow[t]{2}{*}{ Nausea-Score } & weiblich & $8.7(13.4)$ & $9.4(14.9)$ & $9.5(14.8)$ \\
\hline & männlich & $6.1(9.3)$ & $6.7(11.7)$ & $7.4(19.2)$ \\
\hline \multirow[t]{2}{*}{ Oculomotor-Score } & weiblich & $13.3(18.0)^{A}$ & $13.4(16.8)^{\mathrm{B}}$ & $12.9(15.7)^{A}$ \\
\hline & männlich & $8.4(9.9)$ & $8.2(10.1)$ & $8.2(12.2)$ \\
\hline \multirow{2}{*}{ Disorientation-Score } & weiblich & $2.5(5.0)$ & $3.6(6.8)^{C}$ & $4.0(6.5)^{D}$ \\
\hline & männlich & $1.8(3.8)$ & $2.0(3.7)$ & $1.9(4.5)$ \\
\hline \multirow[t]{2}{*}{ Total-Score } & weiblich & $12.4(17.4)^{\mathrm{E}}$ & $13.9(18.3)^{\mathrm{E}}$ & $14.0(17.3)^{\mathrm{E}}$ \\
\hline & männlich & $8.3(10.6)$ & 8.7 (10.9) & $8.9(15.8)$ \\
\hline
\end{tabular}

Legende (MW = Mittelwert, SD = Standardabweichung):

Zweifaktorielle Varianzanalyse mit dreistufigem Messwiederholungsmodell: Geschlecht und Messzeitpunkt interagieren nicht signifikant.

post-hoc Auswertung mittels $t$-Test bei unabhängigen Stichproben: Score unterscheidet sich signifikant unter Berücksichtigung des Geschlechts (= messzeitpunktunabhängiger Einfluss):

\footnotetext{
A Oculomotor-Score $(p<0.05)$

B Oculomotor-Score $(p<0.01)$

c Disorientation-Score: $p<0.05$,

D Disorientation-Score $p=0.09$ (marginal)

E Total-Score $(p<0.05)$
} 
In keiner der statistischen Auswertungen zeigten die ZUGEHÖRIGKEIT ZU EINER

TEILNEHMERGRUPPE (CTRL, EPI-PRÄ, EPI-POST, STROKE, AD; Tab. 3.20.) sowie die REIHENFOLGE DER AUFGABENBEARBEITUNG (Labyrinth/Park vs. Park/Labyrinth; Tab. 3.21.) einen signifikanten Einfluss auf die einzelnen SSQ.Scores.

Tab. 3.20. Deskriptive Statistik für die SSQ-Scores zu den drei Messzeitpunkten, aufgeschlüsselt nach Teilnehmergruppe

\begin{tabular}{|c|c|c|c|c|}
\hline $\begin{array}{l}\text { SSQ-Score } \\
\text { MW (SD) }\end{array}$ & & to & t1 & t2 \\
\hline Nausea-Score & $\begin{array}{l}\text { CTRL } \\
\text { EPI-PRÄ } \\
\text { EPI-POST } \\
\text { STROKE } \\
\text { AD } \\
\text { PAT-GESAMT * }\end{array}$ & $\begin{array}{c}5.7(8.7) \\
10.9(15.3) \\
7.2(8.7) \\
7.4(12.8) \\
6.3(10.7) \\
8.0(12.3) \\
\end{array}$ & $\begin{array}{r}7.2(14.1) \\
10.6(16.5) \\
9.1(10.9) \\
9.1(13.6) \\
5.2(7.5) \\
8.1(12.5) \\
\end{array}$ & $\begin{array}{c}8.9(23.8) \\
9.6(16.3) \\
10.0(12.2) \\
8.7(13.5) \\
5.2(8.6) \\
7.9(12.7)\end{array}$ \\
\hline Oculomotor-Score & $\begin{array}{l}\text { CTRL } \\
\text { EPI-PRÄ } \\
\text { EPI-POST } \\
\text { STROKE } \\
\text { AD } \\
\text { PAT-GESAMT * }\end{array}$ & $\begin{array}{r}8.2(10.2) \\
12.0(13.4) \\
10.6(12.4) \\
12.1(14.9) \\
11.4(18.9) \\
11.6(15.5)\end{array}$ & $\begin{array}{r}8.5(10.6) \\
11.4(13.5) \\
11.4(15.3) \\
12.4(15.8) \\
10.7(15.2) \\
11.3(14.7) \\
\end{array}$ & $\begin{array}{r}8.5(12.2) \\
10.9(13.4) \\
12.1(15.0) \\
12.7(15.0) \\
9.8(5.4) \\
11.1(14.7) \\
\end{array}$ \\
\hline Disorientation-Score & $\begin{array}{l}\text { CTRL } \\
\text { EPI-PRÄ } \\
\text { EPI-POST } \\
\text { STROKE } \\
\text { AD } \\
\text { PAT-GESAMT * }\end{array}$ & $\begin{array}{l}1.6(3.0) \\
2.8(4.4) \\
1.3(2.8) \\
2.7(6.2) \\
2.4(5.4) \\
2.4(4.9) \\
\end{array}$ & $\begin{array}{l}2.7(5.4) \\
2.7(4.2) \\
2.8(7.5) \\
2.7(6.3) \\
2.4(4.0) \\
2.6(5.1) \\
\end{array}$ & $\begin{array}{l}2.9(5.7) \\
3.0(4.4) \\
3.0(6.5) \\
2.9(7.7) \\
2.0(4.1) \\
2.6(5.3) \\
\end{array}$ \\
\hline Total-Score & $\begin{array}{l}\text { CTRL } \\
\text { EPI-PRÄ } \\
\text { EPI-POST } \\
\text { STROKE } \\
\text { AD } \\
\text { PAT-GESAMT * }\end{array}$ & $\begin{array}{r}7.8(10.0) \\
12.9(15.2) \\
9.4(9.7) \\
11.6(16.4) \\
10.5(17.7) \\
11.2(15.5) \\
\end{array}$ & $\begin{array}{r}9.7(13.9) \\
12.4(15.1) \\
12.0(17.3) \\
12.4(16.7) \\
9.7(12.8) \\
11.4(14.9) \\
\end{array}$ & $\begin{array}{r}10.5(18.8) \\
12.2(14.9) \\
12.9(16.9) \\
12.6(17.5) \\
9.0(13.4) \\
11.2(15.1) \\
\end{array}$ \\
\hline
\end{tabular}

Legende: $(M W=$ Mittelwert, $S D=$ Standardabweichung $)$

* PAT-GESAMT = alle Patientengruppen zusammengefasst

Tab. 3.21. Deskriptive Statistik für die SSQ-Scores zu den drei Messzeitpunkten, aufgeschlüsselt nach Reihenfolge der Testaufgaben

\begin{tabular}{|c|c|c|c|}
\hline $\begin{array}{l}\text { SSQ-Score } \\
\text { MW (SD) }\end{array}$ & to & t1 & t2 \\
\hline Nausea-Score & 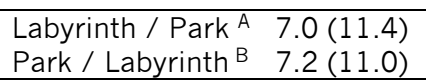 & $\begin{array}{l}8.1(13.4) \\
7.5(12.9)\end{array}$ & $\begin{array}{l}8.2(18.9) \\
8.3(16.5)\end{array}$ \\
\hline Oculomotor-Score & $\begin{array}{ll}\text { Labyrinth / Park } & 10.3(12.8) \\
\text { Park / Labyrinth } & 10.3(15.0)\end{array}$ & $\begin{array}{r}10.9(12.8) \\
9.7(13.8)\end{array}$ & $\begin{array}{r}9.8(12.8) \\
10.4(14.8)\end{array}$ \\
\hline Disorientation-Score & $\begin{array}{ll}\text { Labyrinth / Park } & 2.1(3.6) \\
\text { Park / Labyrinth } & 2.1(4.8) \\
\end{array}$ & $\begin{array}{l}2.3(3.6) \\
2.9(6.3)\end{array}$ & $\begin{array}{l}2.4(4.2) \\
3.0(6.4)\end{array}$ \\
\hline Total-Score & $\begin{array}{lr}\text { Labyrinth / Park } & 9.9(12.5) \\
\text { Park / Labyrinth } & 10.0(14.9)\end{array}$ & $\begin{array}{l}10.9(13.0) \\
10.6(15.8)\end{array}$ & $\begin{array}{l}10.4(15.9) \\
11.4(17.2)\end{array}$ \\
\hline
\end{tabular}

Legende (MW = Mittelwert, SD = Standardabweichung):

A Reihenfolge: 1. Labyrinth (t0/t1), 2. Park (t1/t2)

B Reihenfolge: 1. Park (t0/t1), 2. Labyrinth (t1/t2) 


\subsection{Untersuchung der SSQ-Scores auf Interaktionseffekte}

In einem weiteren Auswertungsschritt der Simulator-Sickness-Daten wurde ein multifaktorielles Auswertungsmodell entworfen, um Interaktionseffekte zwischen unabhängigen Variablen zu untersuchen.

Als abhängige Variablen wurden die Simulator-SicknessScores (Nausea-, Oculomotor-, Disorientation und Total-Score) in einem dreistufigen Messwiederholungsmodell untersucht. Für eine multifaktorielle Analyse der Simulator-Sickness-Daten wurden - soweit zulässig einzelne Faktoren vereinfacht, um hinreichende Stichprobenzahlen in den jeweiligen Faktorstufenkombinationen zu erhalten.

Das Geschlecht wurde als Faktor beibehalten. Die einzelnen Teilnehmergruppen wurden in 'Gesunde Probanden' und 'Patienten' unterteilt. Für die Anzeigemodalität wurde die Kategorisierung 'immersiv' vs. 'non-immersiv' gewählt (s.a. Definition Kap. 1.2.1.). Das HMD, das mit seinem ,Brillen'-Charakter das Gesichtsfeld des Teilnehmers weitgehend einnimmt, wurde als immersiv eingestuft. Demgegenüber wurden das Projektionssystem und der Monitor als nicht-immersive Anzeigemodalitäten bewertet, da der Teilnehmer die Anzeigeflächen zwar betrachtet, jedoch gleichzeitig die Umwelt (den Raum) wahrnehmen kann. Diese Einteilung erschien auch hinsichtlich der Einzelvergleiche der Modalitäten (siehe Kap. 3.5.3.) gerechtfertigt.

Die SSQ-Scores (abhängige Variablen: SSQ Total-, Nausea-, Oculomotor- und DisorientationScore) wurden in einer Varianzanalyse mit dreistufigem Messwiederholungsmodell untersucht, mit folgenden Faktoren:

- $\quad$ Art der Anzeigemodalität (immersiv vs. non-immersiv)

- Geschlecht (weiblich vs. männlich)

- Gruppe (Patienten vs. Gesunde Probanden)

- Messzeitpunkt (to, t1, t2)

Somit ergab sich eine 4-faktorielle Varianzanalyse mit 3 Zwischensubjektfaktoren (Gruppe, Geschlecht, Anzeigemodalität), jeweils 2-fach gestuft, und einem 3-fach-gestuften Messwiederholungsfaktor.

Tab. 3.22. Fallverteilung zur multifaktoriellen Analyse

\begin{tabular}{|c|c|c|c|c|}
\hline \multirow[b]{2}{*}{ Gruppe } & \multirow[b]{2}{*}{ Geschlecht } & \multicolumn{2}{|c|}{ Art der Anzeigemodalität } & \multirow[b]{2}{*}{ Gesamt } \\
\hline & & Immersiv & Non-immersiv & \\
\hline \multirow[t]{2}{*}{ Gesund } & Weiblich & 18 & 11 & 29 \\
\hline & Männlich & 27 & 16 & 43 \\
\hline \multirow[t]{2}{*}{ Patienten } & Weiblich & 21 & 27 & 48 \\
\hline & Männlich & 17 & 57 & 74 \\
\hline Gesamt & & 83 & 111 & 194 \\
\hline
\end{tabular}


Bei der statistischen Analyse wurden keine signifikanten Interaktionen einzelner Faktoren dritter oder vierter Ordnung beobachtet. Weiterhin zeigten sich keine Interaktionen innerhalb der Zwischensubjektfaktoren (Anzeigemodalität, Gruppe, Geschlecht). Ähnlich der vorherigen Analyse (Kap. 3.5.4.3.2.) ließen sich jedoch als höchstrangiger Effekt deutliche Interaktionen zwischen Messzeitpunkt und dem Zwischensubjektfaktor Art der Anzeigemodalität nachweisen.

Hier ergaben sich signifikante Interaktionen für den Total-Score $[F(2,372)=5.756, p<0.01$; $\mathrm{n}=194]$, den Nausea-Score [ $\mathrm{F}(2,372)=3.929, \mathrm{p}<0.05 ; \mathrm{n}=194]$ sowie den Disorientation-Score $[F(2,372)=6.190, p<0.01 ; n=194]$. Für den Oculomotor-Score ließ sich ein marginal signifikanter Einfluss der Art der Anzeigemodalität im Testverlauf nachweisen $[F(2,372)=3.025, p=0.05 ; n=194]$. Anhand der in Tab. 3.23. dargestellten Mittelwerte ist $z u$ erkennen, dass die immersive Anzeigemodalität (HMD) einen Anstieg aller Scores im Vergleich zu den nicht-immersiven Verfahren im Gesamtverlauf bewirkte. In einer post-hoc Analyse wurde untersucht, inwiefern die aufgeführten Effekte sich auf einen einzelnen Testabschnitt beschränkten (Aufgabe 1 entspricht to vs. t1, Aufgabe 2 entspricht t1 vs. t2).

Für alle Scores wurden für die beiden Testabschnitte zweifaktorielle Varianzanalysen in einem zweistufigen Messwiederholungsmodell durchgeführt (Zwischensubjektfaktor Art der Anzeigemodalität). Hierbei zeigte sich, dass sich eine signifikante Interaktion zwischen Messzeitpunkt und Anzeigemodalität auf die erste Aufgabe beschränkte, für Aufgabe 2 konnten keine signifikanten Interaktionen nachgewiesen werden. Für Aufgabe 1 ergab sich ein Einfluss für den Nausea-Score $[F(1,186)=6.810, p<0.05 ; n=194]$, Oculomotor-Score $[F(1,186)=4.109, p<0.05 ; n=194]$, Disorientation-Score $[F(1,186)=6.306, p<0.05 ; n=194]$ und Total-Score $[F(1,186)=7.955, p<0.01 ; n=194]$.

Geschlecht und Gruppe hatten in der statistischen Untersuchung in keinem Fall Einfluss auf einen Anstieg oder Abfall der einzelnen SSQ-Scores.

Aus Gründen der Übersichtlichkeit sind in Tabelle 3.23. lediglich die SSQ-Scores klassifiziert nach Messzeitpunkt und Art der Anzeigemodalität dargestellt. Die entsprechenden Ergebnisse für Geschlecht und Gruppe erschienen bereits in den Tabellen 3.19. und 3.20.. 
Tab. 3.23. Deskriptive Statistik für die SSQ-Scores zu den drei Messzeitpunkten, unterteilt nach immersiven und non-immersiven Anzeigemodalitäten

\begin{tabular}{|c|c|c|c|c|}
\hline $\begin{array}{l}\text { SSQ-Score } \\
\text { MW (SD) }\end{array}$ & Art der Anzeige & to & t1 & t2 \\
\hline \multirow[t]{2}{*}{ Nausea-Score ${ }^{\mathbf{A}}$} & Immersiv & $6.9(11.1)^{D}$ & $9.4(16.2)$ & $10.8(23.9)$ \\
\hline & Non-immersiv & $7.3(11.3)$ & $6.5(10.0)$ & $6.4(10.5)$ \\
\hline \multirow[t]{2}{*}{ Oculomotor-Score } & Immersiv & $9.6(11.2)^{\mathrm{E}}$ & $10.8(12.9)$ & $11.1(14.2)$ \\
\hline & Non-immersiv & $10.9(15.6)$ & $9.9(13.7)$ & $9.4(13.6)$ \\
\hline \multirow[t]{2}{*}{ Disorientation-Score $^{\mathbf{B}}$} & Immersiv & $1.4(2.6)^{\mathrm{F}}$ & $2.9(6.0)$ & $3.3(6.1)$ \\
\hline & Non-immersiv & $2.6(5.2)$ & $2.4(4.5)$ & $2.3(5.0)$ \\
\hline Total-Score $^{\mathbf{C}}$ & $\begin{array}{l}\text { Immersiv } \\
\text { Non-immersiv }\end{array}$ & $\begin{array}{c}8.9(10.8)^{\mathrm{G}} \\
10.0(13.8)\end{array}$ & $\begin{array}{r}11.9(16.2) \\
9.9(12.1)\end{array}$ & $\begin{array}{r}13.0(19.9) \\
9.4(13.5)\end{array}$ \\
\hline
\end{tabular}

Legende (MW = Mittelwert, SD = Standardabweichung):

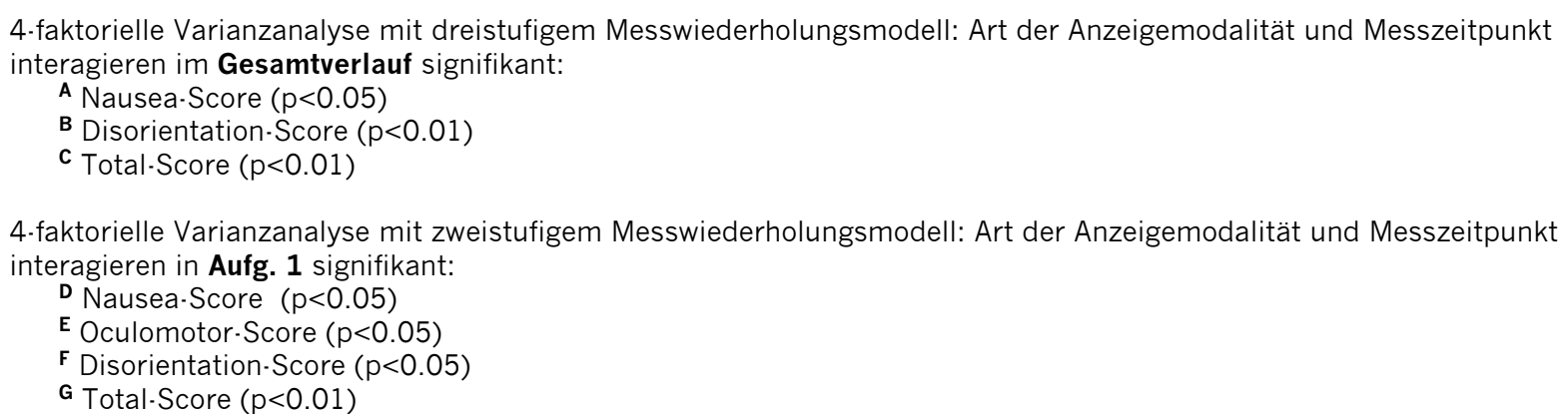

\subsubsection{Zusammenfassung}

In die klinische Studie wurden 215 Testteilnehmer aufgenommen, darunter Kontrollprobanden (CTRL, $n=75$ ), prä-operative (EPI-PRÄ, $n=40$ ) und post-operative Patienten mit medikamentenrefraktärer Epilepsie (EPI-POST, $n=25)$, Patienten mit Z.n. Schlaganfall (STROKE, $n=27$ ) sowie Patienten mit V.a. Alzheimer-Demenz (AD, $n=48)$. Die Testpersonen wurden einer Anzeigemodalität zugeordnet und bearbeiteten bis zu drei Testaufgaben.

In der Benutzerevaluation gaben 10 von 215 Probanden Probleme mit der Gerätebedienung an, diese Gruppe unterschied sich jedoch hinsichtlich Alter, Computervorkenntnissen, Anzeigemodus und Teilnehmergruppe nicht signifikant vom übrigen Kollektiv. Ein Testabbruch wurde in 17 Fällen verzeichnet, davon waren unerwünschte Nebenwirkungen drei Mal die Ursache. Mit der Verwendung eines neuen Head Mounted Display (Sony Glasstron) konnte der Tragekomfort wesentlich verbessert werden. Bei der Beurteilung des Realismus zeigte sich, dass der Park gegenüber den anderen Testaufgaben realistischer eingeschätzt wurde. Die Park-Testaufgabe zeigte auch bei der Analyse der Immersion signifikant bessere Beurteilungen. Die Anzeigemodalität hatte weder einen Einfluss auf den Realismus noch auf die Immersion. Wie in der präklinischen Studie korrelierten die Beurteilung von Immersion und Realismus signifikant miteinander.

Die Untersuchung der erfassten Nebenwirkungen erfolgte wie in der Vorstudie mittels Simulator Sickness Questionnaire. 1.4\% der Testpersonen (3 von 215) brachen die Testung 
wegen Nebenwirkungen ab, alle Abbrecher stammten aus der HMD-Gruppe. Die interferenzstatistische Untersuchung der SSQ-Scores wurde an 194 Probanden durchgeführt.

Im ersten Schritt wurden die SSQ-Scores auf signifikante Veränderungen im Testverlauf untersucht. Als Ausgangsbasis dienten hierfür die vor der VR-Exposition erhobenen SSQ-Werte. Ein signifikanter Anstieg im Gesamtverlauf der Testung wurde lediglich für den DisorientationScore nachgewiesen. In der post-hoc Analyse zeigte sich, dass die signifikanten Änderungen bereits im ersten Testabschnitt auftraten und danach kein weiterer Anstieg zu verzeichnen war. Für die übrigen Scores ließen sich keine signifikanten Änderungen im Testverlauf nachweisen. In der weiteren Analyse wurden mögliche Einflussfaktoren auf die SSQ-Scores im Testverlauf untersucht. Für Alter, Reihenfolge der Aufgabenbearbeitung und Zugehörigkeit zu einer Gruppe (Gesunde, Patientengruppen) konnte kein relevanter Einfluss auf die SSQ-Scores nachgewiesen werden. Allerdings wiesen weibliche Testteilnehmer unabhängig vom Testverlauf signifikant höhere Werte für den Oculomotor-, Disorientation und Nausea-Score auf. Ein signifikanter geschlechterabhängiger SSQ-Score-Anstieg oder -Abfall zeigte sich nicht.

Eine deutlicher Einfluss der Anzeigemodalität auf die Messzeitpunkte wurde statistisch nachgewiesen. Für den Nausea-, Disorientation- und Total-Score konnte gezeigt werden, dass die Gruppe der HMD-Benutzer einen signifikant stärkeren Anstieg der Werte über den Testverlauf bot als die Monitor- und Projektionsgruppe. Der signifikante Einfluss beschränkte sich jedoch auf den ersten Testabschnitt, im zweiten Testabschnitt veränderten sich die Werte der HMD-Gruppe nicht signifikant im Vergleich zu den anderen Anzeigemodalitäten. Diese Beobachtung der Unterschiede konnte in der multifaktoriellen Analyse noch akzentuiert werden. Hier wurden zur Untersuchung auf Interaktionseffekte höherer Ordnung die Anzeigemodalitäten in ,immersiv' (HMD) und ,nicht-immersiv' (Monitor/ Projektion) kategorisiert, als weitere Faktoren wurden Geschlecht, Gruppe (Patient/Gesund) und Messzeitpunkt herangezogen. Signifikante Interaktionen wurden wiederum zwischen Anzeigemodalität und Messzeitpunkt beobachtet, im ersten Testabschnitt stiegen sämtliche SSQ-Scores der immersiven Gruppe signifikant stärker als die der non-immersiven. Weitere höhergradige Interaktionen zwischen Faktoren wurden nicht beobachtet. 


\section{Diskussion}

\subsection{Planung und Aufbau eines interdisziplinär nutzbaren, medizinischen VR-Labors}

Mit der Analyse existierender medizinischer Virtual-Reality-Anwendungen [A.1] ${ }^{*}$ aus den Jahren 1996/1997 konnten Ausgangsdaten für die Laborplanung gewonnen werden.

Anhand einer Auswertung von Publikationen medizinischer VR-Anwendungen wurden technische Komponenten identifiziert, die in einem Großteil der untersuchten Projekte zum Einsatz kamen. Bei den visuellen Anzeigesystemen waren $85 \%$ handelsübliche Bildschirme, z.T. mit Stereoskopie-fähigen Shutter-Brillen, sowie Head Mounted Displays. Stereoskopische Verfahren wurden insgesamt nur in 30\% angewandt. Bei den Eingabesystemen wurde in der Analyse zwischen der Anwendungsart ,Arzt' und ,Patient' differenziert. Bei der ärztlichen Anwendung interagierte dieser fast ausschliesslich mit Tastatur, Tracking-Systemen sowie individuell angefertigten Werkzeugen (z.B. mit Sensoren bestückten Endoskopen). Für den Patienten als Anwender zeigte sich ein heterogeneres Bild: Neben Tastatur und TrackingSystemen wurden häufig Joystick und Datenhandschuhe verwendet. Bei den Steuer- und Kontrollrechnern fand man eine gleichmäßige Verteilung über die drei Hardware-Klassen HighEnd-Rechner/ Graphikworkstation/ Standard-PC. Im Bereich der Betriebssysteme dominierte Unix deutlich mit $61 \%$ der eingesetzten Systeme.

Auffällig war, dass die meisten VR-Anwendungen nicht mit VR-spezifischer Technik betrieben wurden:

- häufigstes visuelles Anzeigesystem waren herkömmliche Bildschirme,

- mehr als zwei Drittel der Anzeigesysteme wurden monoskopisch verwendet,

- $\quad 39 \%$ der Eingabesysteme waren VR-untypisch (Tastatur, Maus, Joystick).

Im Hinblick auf Definition und Anspruch ,virtueller Realität' entsprach die Ausstattung zu einem großen Teil konventioneller Computertechnik. Dies hat unterschiedliche Gründe:

- hohe Anschaffungskosten für VR-Hardware (s.a. Pugnetti et al. 1998),

- zurückhaltender Einsatz der Technologie wegen unzureichend evaluierter Nebenwirkungen (Whalley 1995, Ring 1998),

- nicht gesicherte Vorteile der Virtual Reality gegenüber konventioneller PC-Technologie und Standardverfahren für viele Aufgabenstellungen (Rizzo et al. 1998a).

\footnotetext{
* die in eckige Klammern gesetzten Ziffern beziehen sich auf die in Kapitel 1.4 (Seite 19-23) definierten Ziele und Hypothesen.
} 
Jede Technikanalyse - auch die hier vorgestellte und Anfang 1998 durchgeführte Untersuchung - bleibt bei einer sich rasant weiterentwickelnden Technologie nur eine Momentaufnahme. Dennoch konnten wichtige Basiskomponenten für ein interdisziplinäres, medizinisches VR. Labor identifiziert werden.

Anhand dieser Analyse sowie der Marktübersicht wurde die Grundausstattung des VR-Labors [A.2] vorgenommen. Als Steuer- und Kontrollrechner wurde eine Windows NT-Workstation ausgewählt, die gegenüber konkurrierenden UNIX-Systemen gleichwertige Leistungsmerkmale bei geringeren Kosten bot. Bei den Anzeigeverfahren wurden drei Geräte ausgewählt: ein Standardmonitor mit einem Shutter-Brillen-System zur stereoskopischen Visualisierung, ein monoskopisches Einwand-Projektionssystem sowie ein Head Mounted Display mit monoskopischem als auch stereoskopischem Anzeigemodus. Damit standen zum einen nonimmersive Anzeigeverfahren (Monitor, Projektion) als auch ein immersives System (HMD) für die Breite medizinischer VR-Anwendungsmöglichkeiten zur Verfügung. Als einziges VRspezifisches Eingabesystem wurde ein Trackingsystem beschafft. Dieses war für den Einsatz mit dem HMD zum Head Tracking vorgesehen.

Die eingesetzte VR-Technologie im Bereich der Medizin hat sich in den letzten Jahren gewandelt: anhand der Proceedings der Tagung ,Medicine Meets Virtual Reality' 2000 und 2001 (Westwood et al. 2000, 2001) lassen sich die technischen Veränderungen seit Abschluss der Konzeptionsphase dieser Arbeit nachvollziehen. Bezugnehmend auf die in dieser Arbeit gewählten Analyseschwerpunkte soll hier in einer nicht-quantitativen Übersicht der aktuelle Stand wiedergegeben werden.

Bei den verwendeten Rechnersystemen spiegelt sich der in der gesamten IT.Welt zu beobachtende Trend wider, für Graphikanwendungen verstärkt auf Personal Computer (PC) mit leistungsfähigen Graphikkarten statt auf Unix-basierte Workstations (z.B. SGl Octane) zurückzugreifen. Teilweise werden Standard-PCs in Netzwerken zu sogenannten ,Clustern' zusammengefasst, um anspruchsvolle Rechen- oder Visualiserungsaufgaben zu bewältigen (Blanquer et. al. 2000). Inzwischen existieren PC-betriebene Lösungen für das hoch aufwändige Anzeigesystem CAVE (Pimentel de Sampaio Goes et al. 2001) oder zur Neuronavigation (Akatsuka et al. 2000). Bei den Betriebssystemen zeigt sich entsprechend der überwiegende Einsatz der Windows-Plattformen und verschiedener Unix-Derivate, neu dabei LINUX für Personal-Computer. Erwähnenswert ist, dass bei zahlreichen Web-basierten Anwendungen das verwendete Betriebssystem keine Rolle mehr spielt (z.B. John und Phillips 2000). Die Eingabesysteme diversifizieren sich in zwei Richtungen: auf der einen Seite stehen die Standardeingabesysteme (Maus, Tastatur) für Forschungs- und Web-basierte Anwendungen, andererseits werden anwendungsspezifische Systeme - v.a. im Trainingsbereich - für realistische Interaktionen eingesetzt (Tasto et al. 2000). Keine relevanten Änderungen zeigen sich bei den visuellen Anzeigeverfahren. 
Als erstes Projekt im Labor wurde im Rahmen dieser Arbeit ein neuropsychologisches Testsystem realisiert. Die Vorgehensweise orientierte sich dabei an dem in Kap. 3.2. vorgestellten Laborkonzept [A.3]. Die technische Basisausstattung erwies sich als ausreichend für das neuropsychologische Projekt. Eine weitgehende Herauslösung des Projektes aus dem VR-Labor folgte mit Beginn der DFG-geförderten klinischen Evaluation.

Für die Evaluation des neuropsychologischen Testsystems im klinischen Bereich wurde aus praktischen Gesichtspunkten ein zusätzlicher VR-Arbeitsplatz eingerichtet, sodass ein weiterer Graphikrechner angeschafft werden musste. Leistungstests zur Auswahl eines geeigneten Rechners zeigten, dass innerhalb des Entwicklungszeitraums des Testsystems (etwa 1 Jahr) sehr leistungsfähige Alternativen zu den Workstations im PC-Bereich aufkamen. Die Tendenz, erweiterte Standard-PC-Systeme auch für anspruchsvollere VR-Anwendungen einzusetzen, zeigt sich z.B. auch am Projekt, Personal Immersion“ des Fraunhofer Instituts IAO Stuttgart. VR-Anwendungen mit Mehrwandprojektionssystemen galten lange aufgrund der hohen Leistungsanforderungen als die klassische Domäne der High End-Rechner (z.B. SGl Onyx2). Dort konnte gezeigt werden, dass inzwischen mit vernetzten PCs auf Linux-Basis bei gleicher Leistungsfähigkeit im Vergleich zu High End-Rechnern Kosten von bis zu 80\% eingespart werden können (FHG-IAO 2001).

Zur Zeit werden im VR-Labor neben dem neuropsychologischen Testsystem weitere Projekte bearbeitet. In einem Kooperationsprojekt mit der Abteilung Kieferchirurgie der Universität Göttingen wird eine VR-Anwendung für die intraoperative Visualisierung von 3D-Ultraschall mit MRT-Matching entwickelt (Verhey et al. 2002, Ludwig u. Verhey 2000). In einem internen Projekt der Abt. Medizinische Informatik wurde der Prototyp eines Krankenhausnavigationssystems als virtueller Wegweiser entwickelt. Weiterhin wurden auf der Grundlage der neuropsychologischen Testumgebung Animationssequenzen als Stimulus für die funktionelle MRT erstellt (Schwerpunkt Neuropsychologie, Abt. Psychiatrie, Universität Göttingen). Das VR-Labor wird den Anforderungen laufender Projekte entsprechend ständig aktualisiert. Erweitert wurde die Ausstattung neben neueren PC-Graphiksystemen z.B. um eine medizinische Bildverarbeitungssoftware (3D Slicer, Massachusetts Institute of Technology) zur Segmentierung und Visualisierung radiologischer Daten.

Das Konzept einer VR-Kompetenzeinrichtung für Interessenten ohne eigenes technisches KnowHow mit Interesse an der Verwendung von VR-Technologien wird an anderen Orten bereits seit längerem erfolgreich praktiziert, so z.B. am Competence Center Virtual Reality des Fraunhofer Instituts für Arbeitswirtschaft und Organisation (IAO) in Stuttgart. Als Vorteil wird auch dort hervorgehoben, dass mit einem ,Kompentenzzentrum“ die relativ teure Technik und das Fachwissen durch verschiedene Gruppen oder Industriepartner verwendet werden können (Riedel 1997). 


\subsection{Technische Entwicklung eines VR-gestützten neuropsychologischen Test-Systems}

Das neuropsychologische Testsystem wurde in mehreren Stufen entwickelt, dieses umfasste auch eine Prototypenerstellung. Am Ende des Entwicklungsprozesses stand ein funktionsfähiges Testsystem, welches dem Testleiter ermöglichte, komfortabel ohne vertieftes technisches Wissen verschiedene VR-Konfigurationen zu bedienen. Mit dem Testsystem einsetzbar waren alle im VR-Labor verfügbaren Anzeigeverfahren (HMD, Monitor, Projektion), also sowohl immersive als auch non-immmersive Systeme.

Für die präklinische Studie standen als Testumgebungen ein Labyrinth und eine Parklandschaft zum räumlichen Lernen zur Verfügung, zur Durchführung der klinischen Studie wurde zusätzlich eine Aufgabe zu exekutivem Lernen implementiert. Zur benutzerfreundlichen Anwendung der Testaufgaben und Verwaltung der Probanden wurde ein Testadministrationsprogramm entwickelt. Werkzeuge zur quantitativen (Fehler, Zeit) und qualitativen (Wiedergabemodus der Testdurchführung) ermöglichten die Auswertung von Testleistungen.

Besonderes Augenmerk bei der Entwicklung lag auf einer einfachen, zielgruppenorientierten Bedienbarkeit der Testaufgaben [B.1]. Mit der Zielgruppe neurologischer und psychiatrischer Patienten höheren Lebensalters wurden bereits im Prototypenstadium verschiedene Interaktionsarten zur Navigation in der virtuellen Umgebung erprobt. Hier zeigte sich als wesentlich, dass eine teilweise geführte Navigation auf definierten Pfaden in der virtuellen Umgebung eine Vereinfachung der Bedienbarkeit nach sich zog und eine deutlich geringere Nauseogenität besaß.

Im Vergleich zu den konventionellen Paper \& Pencil-Tests zeigte die entwickelte VR-Anwendung vor allem drei Vorteile für das visuell-räumliche Lernen:

- die Testperson bearbeitet nicht mehr eine abstrakte zweidimensionale Aufgabe aus der Vogelperspektive (z.B. Aufsicht auf ein Labyrinth), sondern bewegt sich aus der IchPerspektive innerhalb einer dreidimensionalen Umgebung [B.3].

- Die Anzahl der Freiheitsgrade, die eine Testperson benutzen kann, ist in der virtuellen Umgebung deutlich höher (Perspektivenwechsel, Bewegung, realistische Geschwindigkeit, wirklichkeitsnahe Darstellung).

- Eine automatische Auswertung von Fehlerindizes kann durchgeführt werden, weiterhin können anhand der aufgezeichneten Daten neben Standardauswertungen nachträglich weitere Verhaltensparameter berechnet werden [B.2].

Insbesondere die ersten beiden Punkte sind ein wesentliches Argument für die Anwendung einer virtuellen Testumgebung für die Erfassung des visuell-räumlichen Lernens. Nur durch 
diese Charakteristika der Testumgebung ist eine valide Erfassung des visuell-räumlichen Lernens überhaupt möglich. Es wird daher angenommen, dass VR-Anwendungen für zahlreiche neuropsychologische Fragestellungen eine höhere Sensitivität, Testvalidität und damit einen besseren prädiktiven Wert für funktionelle Einschränkungen im Alltag besitzen (Pugnetti et al. 1998). Ein systematischer Nachweis hierzu steht jedoch aus und soll Gegenstand weiterer Untersuchungen sein.

Ein bildlicher Realismus der Testaufgaben war im Rahmen der technischen Möglichkeiten unserer Studie nicht intendiert. Trotz, virtueller' Bewegung ist ein Kritikpunkt bezüglich der ökologischen Validität von neuropsychologischen computergestützten Tests das Fehlen einer körperlichen Bewegung und damit der Integration sensorischer und motorischer Informationen (Wartenberg et al. 1998). Technisch aufwändigere Ansätze zur Lösung dieses Problems sind z.B. mit der Verwendung eines stationären Fahrrads (van Veen et al. 1998) oder eines Laufbandes (Witmer und Kline 1998) gemacht worden. Es ist jedoch festzuhalten, dass solche aufwändigen und kostspieligen Installationen den Rahmen dieser ersten Studie überschritten hätten.

\subsection{Präklinische und klinische Evaluationsstudie}

Die Evaluation des Testsystems wurde in zwei Phasen vorgenommen, einer präklinischen sowie einer klinischen Studie. Die präklinische Studie (im folgenden aufgeführt als PILOT-Studie) wurde mit 22 gesunden Probanden durchgeführt, als Anzeigemodalitäten wurden ein Projektionssystem sowie ein Head Mounted-Display verwendet. Im Anschluss an die PILOT. Studie wurden am Testsystem Modifikationen vorgenommen: wesentlich ist hier die Auswahl eines komfortableren HMD sowie die Überarbeitung der virtuellen Umgebungen (Realismus, Aufgabenkomplexität). An der folgenden klinischen Studie (KLIN-Studie) nahmen 215 Personen teil, darunter gesunde Testprobanden sowie Patienten mit verschiedenen neurologischen Krankheitsbildern.

\subsubsection{Praktikabilität des Testsystems [C.1]}

Das VR-Testsystem und die Aufgabenstellung konnten generell von den Probanden nach kurzer Trainingsphase (max. 6 Minuten) ohne Bedienungsprobleme beherrscht werden. Die kurze und ausreichende Trainingsdauer ist insofern hervorzuheben, als für vergleichbare VRNavigationsaufgaben bei unterschiedlicher technischer Realisierung Trainingszeiten von bis zu 75 Minuten angegeben werden (Waller et al. 1998).

In der PILOT-Studie verneinten alle Probanden Schwierigkeiten mit der Bedienung des Testsystems (Projektion mit Tastatur, anschließend HMD ,VirtualResearch V8' mit Tracking. System/ Maus). Das circa zwei Kilogramm schwere HMD wurde in etwa der Hälfte der Fälle als beklemmend bezeichnet, in der Nachbefragung bemängelten die Probanden Gewicht und 
Komfort des Gerätes. Ein Proband konnte die HMD-Testung nicht durchführen, da dieses nicht an die Kopfgröße anzupassen war. Als hinderlich bei der Bewegung und Kopfdrehung wurde das mit dem HMD verbundene Strom-/Datenkabel empfunden. Eine deutliche Präferenz für ein Anzeigeverfahren (HMD vs. Projektion) bestand allerdings nicht.

Die Teilnehmer der klinischen Studie wurden im Gegensatz zur PILOT-Studie nur einem Anzeigeverfahren zugeordnet (HMD ,Sony Glasstron' / Tracking.System / Maus oder Projektion oder Monitor mit Tastatur / Joystick). Aufgrund der Teilnehmerrückmeldung der PILOT-Studie wurde für die klinische Studie ein leichteres HMD-System verwendet (Sony Glasstron, $150 \mathrm{~g}$ ). Die Teilnehmer wurden hinsichtlich der eingesetzten Anzeigeverfahren nicht randomisiert. Die Probanden hatten die Gelegenheit zwischen den Anzeigeverfahren zu wählen, wobei jüngeren Probanden eher zum HMD, älteren eher zu Monitor / Projektion geraten wurde. Die Auswahl des Gerätes unterlag damit einem deutlichen Bias, dieses ist bei den folgenden Ergebnissen zu berücksichtigen.

17 Teilnehmer wechselten die Anzeigemodalität während der max. 3 Testaufgaben, unter anderem aus technischen oder Komfortgründen. Statistisch unterschied sich die WechslerGruppe nicht in ihrer Zusammensetzung bezüglich der anfangs ausgewählten Anzeigemodalität, des Alters und der Computervorkenntnisse von den Nicht-Wechslern. Es befanden sich jedoch signifikant überzufällig viele EPI-POST. und STROKE-Patienten unter den Wechslern. Dies ist durchaus als indirekter Hinweis zu beachten, dass bei klinischen Patientengruppen - insbesondere bei Patienten mit strukturellen Läsionen - im Unterschied zu gesunden Probanden eine größere Problematik bei der Verwendung immersiver Anzeigeverfahren zu sehen ist. Lediglich 6 von 78 HMD-Benutzern gaben eine Unbehaglichkeit bei der Geräteverwendung an, Probleme mit der Gerätebedienung gaben 10 von 215 Probanden an, wobei sich diese in keinem Merkmal signifikant vom Rest-Kollektiv unterschieden.

Vergleichbare Studien, in denen eine Benutzerbewertung von Navigation und Anzeigesystem bei einer neurowissenschaftlichen Fragestellung durchgeführt wurde, sind uns nicht bekannt. In einer VR-Navigationsaufgabe (Morris Water Maze Test) mit 12 gesunden Probanden und 12 Patienten (Zustand nach Schädel-Hirn-Trauma) mittels Monitor und Joystick ermittelten Skelton et al. (2000), dass weder mit der Aufgabe noch der Navigation Probleme bestanden. Rizzo et al. (2002) wiesen für eine VR-gestützte neuropsychologische Testaufgabe darauf hin, dass Kinder keine Probleme mit der HMD-Verwendung hätten.

Während in der PILOT-Studie für die positive Benutzerbewertung der Navigation Bildungsstand und Computerwissen der 22 Probanden als Begründung ins Feld geführt werden können, zeigte sich in der KLIN.Studie, dass auch mit einer älteren Teilnehmerpopulation und Patienten weniger als 5\% der Teilnehmer Probleme mit der Gerätebedienung angaben (10 von 215 Teilnehmern). Leider ergab die statistische Auswertung der Gruppe mit 
Bedienungsproblemen keinen Hinweis auf ein spezielles Problemfeld. An dieser Stichprobe konnte kein signifikanter Zusammenhang zwischen Alter, Geschlecht, Anzeigemodalität, Computervorkenntnissen oder Gruppenzugehörigkeit und den Bedienungsproblemen nachgewiesen werden.

Inwiefern hinsichtlich der Bedienungsprobleme Verbesserungsmöglichkeiten bestehen, kann anhand der Befragungsdaten und unsystematischer Beobachtung der Probanden nicht beantwortet werden: wenn auch aufwändig, wird eine strukturierte Benutzerbeobachtung und Einbindung zukünftiger Benutzer während der Entwicklung als sehr effektives Mittel gesehen, die Schnittstelle zwischen Benutzer und Anwendung zu optimieren (Gomoll 1990, Kushniruk et al. 1997). Sicher wünschenswert wäre eine kabellose Interaktion bei Probanden, die das HMD verwenden.

\subsubsection{Benutzerbewertung von Realismus und Immersion [C.2]}

Die Bedeutung der verwendeten Anzeigemodalität (HMD, Projektion, Monitor) sowie des Testinhalts (Testaufgabe Park, Labyrinth, ggf. Exekutiv) auf die Bewertung von Realismus und Immersion wurden zur Überprüfung der Hypothese 1 untersucht.

In der PILOT-Studie zeigte die Anzeigemodalität (HMD vs. Projektion) auf die Bewertung des Realismus der VR.Umgebung keinen signifikanten Einfluss. Hinsichtlich der Immersion wies das HMD jedoch eine signifikant bessere Bewertung auf. In der KLIN-Studie konnte weder ein Einfluss der Anzeigemodalität auf die Bewertung des Realismus noch auf die Immersion nachgewiesen werden.

Bei der Untersuchung des Einflusses von Testinhalt auf Immersion und Realismus zeigten sich dagegen deutliche Interaktionen: bei der Bewertung des Realismus der virtuellen Umgebung in der KLIN-Studie wurde die Park-Aufgabe signifikant realistischer eingestuft als Labyrinth- und Exekutiv-Aufgabe. Die Bewertung des Immersionsgefühls lag ebenfalls signifikant höher im Park.

Sowohl in der PILOT. als auch in der KLIN-Studie zeigte sich eine positive Korrelation von Immersion mit der Bewertung des Realitätsgrads der virtuellen Umgebung.

In der Forschungsagenda von Stanney et al. (1998) werden zahlreiche Einflussfaktoren auf die Immersion als Gefühl der Anwesenheit in einer virtuellen Umgebung aufgeführt. In der vorliegenden Arbeit wurden ein Teil dieser Faktoren in den drei Anzeigemodalitäten konstant gehalten (z.B. Anzahl der sensorischen Modalitäten, Mono./ Stereoskopie, Bildwiederholungsrate).

In der Literatur wird das HMD per definitionem als immersives Anzeigeverfahren bezeichnet. Wesentliche Unterschiede des HMD gegenüber anderen Anzeigeverfahren (z.B. Monitoren oder Projektionsverfahren) sind das Head-Tracking-System sowie die konstruktionsbedingte 
Abschirmung des Benutzers von anderen visuellen Einflüssen im Raum (Hendrix und Barfield 1996, Witmer und Singer 1998). In der KLIN-Studie erreichte das HMD jedoch in der Teilnehmerbeurteilung der Stichprobe $(n=215)$ keine signifikant höhere Immersion als die konventionellen Anzeigeverfahren. In der PILOT-Studie, in der den 22 Probanden ein direkter Vergleich zwischen HMD und Projektion ermöglicht wurde, erreichte das HMD allerdings bessere Immersionsbeurteilungen. Insgesamt lässt sich aus den Ergebnissen keine deutliche Überlegenheit des HMD ablesen. Die Ursache hierfür mag vielleicht darin liegen, dass durch die teilgeführte Bewegung in der Testumgebung und die wenig interaktive Testaufgabe die grundsätzlichen Vorteile des HMD nicht zum Tragen kamen.

In der vorliegenden Studie wurde - wie auch in anderen Untersuchungen (z.B. Deisinger at al. 1997) - die Immersion als einzelnes Fragebogenitem abgefragt. Inzwischen wurden Empfehlungen ausgesprochen, dass bei dezidierten Fragestellungen zu Immersion und ,Sense of Presence' neben ausführlichen Fragebögen (z.B. Lessiter et al. 2001) auch physiologische Variablen wie Hautwiderstand oder Herzfrequenz und Verhaltensparameter erfasst werden sollten (Insko 2003).

Der signifikant höhere Realitätsgrad des Parks kann auf die inhaltliche Gestaltung zurückgeführt werden: Technik und Interaktion wurden zwischen den drei Aufgabenarten nicht variiert, jedoch profitiert die Park-Umgebung anscheinend von der Ausgestaltung mit zahlreichen, alltäglichen Objekten (Witmer und Singer 1998). Die signifikante Korrelation von Realismus und Immersion unterstützt die Annahme, dass der Realitätsgrad einer virtuellen Umgebung dazu beiträgt, das Gefühl der Immersion zu steigern (Stanney et al. 1998, Witmer und Singer 1998), obgleich die Ergebnisse hierzu nicht einheitlich sind (Welch et al. 1996). Inwiefern die graphische Komplexität (bildlicher Realismus) und die Hinzunahme weiterer Interaktions- und Anzeigemodalitäten (z.B. Laufband, Geräusche) Einfluss auf die Bearbeitung einer neuropsychologischen Testaufgabe haben, ist nicht bekannt.

\subsubsection{Nebenwirkungen virtueller Realität: Simulator Sickness}

In der präklinischen Pilotstudie (PILOT-Studie) sowie der klinischen Studie (KLIN-Studie) wurde das Auftreten von unerwünschten Nebenwirkungen mittels mehrfach im Testverlauf erhobener Simulator Sickness Questionnaires (SSQ) untersucht sowie Testabbrüche dokumentiert [C.3]. Anhand dieser Daten sollte überprüft werden, inwieweit in dem neuropsychologischen Testsystem ein Einfluss individueller (Alter, Geschlecht, Krankheit) und äußerer (Anzeigesystem) Faktoren bestätigt werden konnte [C.4].

Hinsichtlich der Dokumentation von Nebenwirkungen wird zur Erfassung der subjektiven Symptome der Simulator Sickness Questionnaire (Kennedy und Lane 1993) als weitverbreitetes und geeignetes Mittel angesehen. Eine Vorhersage der individuellen Prädisposition zur Simulator Sickness kann im Gegensatz zu den Kinetosen (Motion History 
Questionnaire) noch nicht geleistet werden, auch standardisierte objektive Messverfahren zur Simulator Sickness fehlen (Stanney et al. 1998).

In der PILOT-Studie konnte gezeigt werden, dass der Projektionsmodus zu keinem Anstieg der SSQ-Scores führte: für den Oculomotor-Score konnte sogar ein signifikanter Abfall nachgewiesen werden. In den darauffolgenden Durchgängen mit HMD kam es jedoch zu einem signifikanten Anstieg des Disorientation-Scores. Aufgrund des Versuchsablaufs ist die Schlussfolgerung, dass das HMD nauseogener als der Projektionsmodus ist, nur eingeschränkt zulässig. Dennoch wird diese These durch die Tatsache, dass sich der einzige reguläre Testabbruch bei Verwendung des HMD ereignete (einer von 21 Probanden $=4.76 \%$ ), unterstützt. Als weiterer Hinweis kann die Tatsache gewertet werden, dass die AbbrecherGruppe erst im HMD-Modus einen deutlichen Anstieg der SSQ-Scores zeigte, während sich diese im Projektionsmodus nicht von der übrigen Gruppe unterschieden.

Mit Einsatz des modifizierten neuropsychologischen Testsystems der KLIN-Studie wurden an einem größeren Teilnehmerkollektiv mögliche Einflussfaktoren auf das Auftreten von Nebenwirkungen untersucht. In den interferenzstatistischen Untersuchungen wurde der Einfluss des Alters, der verwendeten Anzeigemodalität, des Geschlechts, der Zugehörigkeit zu einer Teilnehmergruppe und die Reihenfolge der Aufgabenbearbeitung analysiert. Ohne Einfluss auf die SSQ-Scores blieben Alter, Zugehörigkeit zu einer Teilnehmergruppe (Patient/Gesunde) und die Reihenfolge der Aufgabenbearbeitung.

Die Anzeigemodalität (Monitor, Projektion, HMD) hatte einen signifikanten Einfluss auf einen Anstieg der SSQ-Scores im Testverlauf. Für den Total- und Disorientation-Score zeigte sich, dass das HMD einen signifikanten Einfluss im Sinne eines Score-Anstiegs ausübte; für den Nausea-Score konnten marginal signifikante Einflüsse derselben Art nachgewiesen werden. Bei der Untersuchung der beiden Testabschnitte (Aufgabe 1, Aufgabe 2) zeigte sich, dass der signifikante Anstieg der SSQ-Scores in allen Fällen im Verlauf der ersten Testaufgabe $\left(t_{0} / t_{1}\right)$ eintrat. Dies könnte ein Hinweis für eine Adaptation der Probanden an die VR-Technik nach einer Gewöhnungsphase sein. Projektion und Monitor unterschieden sich nicht in Hinblick auf die SSQ-Scores. Aufgrund dieser Daten konnten in einem weiteren Auswertungsschritt mit der Klassifikation immersive (HMD) vs. non-immersive (Projektion, Monitor) Anzeigemodalität die vorhergenannten Ergebnisse noch akzentuiert werden: die immersive Anzeigemodalität führte zu einem signifikanten (T-, N-, D-Score) oder marginal-signifikanten (O-Score) Anstieg aller SSQ-Scores.

Von den 215 Teilnehmern brachen drei wegen Nebenwirkungen ab (=1.4\%). Diese stammten aus der Gruppe der HMD-Benutzer, darunter zwei gesunde Kontrollprobanden und ein Patient. In Zusammenschau der Ergebnisse der PILOT. und KLIN.Studie kann festgehalten werden, dass Testabbrüche aufgrund von Nebenwirkungen nur bei Verwendung eines HMD verzeichnet 
wurden. Korrespondierend konnte in beiden Studien ein signifikanter Anstieg von einzelnen SSQ-Scores beim HMD-Einsatz aufgezeigt werden, während nicht-immersive Anzeigemodalitäten ohne Einfluss blieben.

Der in den Studien von Naepflin und Menozzi (1998) und Cobb et al. (1999) gewonnene Eindruck, dass im Vergleich von HMD mit einer Monitorkonfiguration das HMD stärker relevante Simulator-Sickness-Symptome hervorruft, konnte in unserer Studie bestätigt werden. Vergleichend konnten jedoch z.B. in verhaltenstherapeutischen Studien mittels VR bei Verwendung eines HMD bei einem Kollektiv von 48 Teilnehmern (Riva 1998: Modifikation des Körperselbstbildes) und 23 Teilnehmern (Garcia-Palacios et al. 2002: Desensibilisierung bei Spinnen-Phobie) keine Abbrecher aufgrund von Nebenwirkungen verzeichnet werden. Auch die hier präsentierten günstigen Daten hinsichtlich nicht-immersiver Anzeigeverfahren lassen sich in der Literatur nicht immer verifizieren. In einer sehr ähnlich konfigurierten kognitionspsychologischen Testaufgabe mit Verwendung eines halb-zylindrischen $180^{\circ}$. Projektionssystems brach einer von 20 Probanden die Testung wegen Nebenwirkungen ab (Riecke et al. 2002). Bei einer Navigationsaufgabe mit einer stereoskopischen Monitorkonfiguration zeigten bis zu 95\% der Probanden einen Anstieg der SSQ-Scores (Stanney und Hash 1998), wobei hier u.a. eine rein passive Navigation verwendet wurde.

In unserer Studie zeigte das Geschlecht einen vom Testverlauf unabhängigen Einfluss auf die SSQ-Scores: für den Total-, Oculomotor- und Disorientation-Score konnte nachgewiesen werden, dass die ermittelten Werte weiblicher Testteilnehmer zu allen Testzeitpunkten über denen der männlichen Teilnehmer lagen, ohne jedoch einen signifikanten Anstieg zu zeigen. Worauf der von uns beobachtete Effekt zurückzuführen ist, kann anhand existierender Erkenntnisse nicht sicher bestimmt werden.

In Übersichtsarbeiten wird ein Geschlechtseffekt im Sinne einer höheren Empfindlichkeit für Kinetosen bei Frauen beschrieben (Kolasinski 1995, 1996), Nichols und Patel (2002) führen in ihrer Zusammenstellung eine Studie auf, in der alle 9 Testabbrecher weiblich waren. Die meisten Studien, die einen Geschlechtseinfluss nachwiesen, bezogen sich allerdings auf die Motion Sickness History oder auf Nachbefragungen, also Angaben, die sich vergangene Ereignisse reflektieren. Für die während eines Bewegungsstimulus erhobenen Werte konnte z.B. kein signifikanter Geschlechtseinfluss gezeigt werden (Jokerst et al. 1999, Park und Hu 1999). Einen signifikant stärkeren Anstieg des Total-Scores bei weiblichen Testteilnehmern mit ähnlichen Ausgangswerten wie die männliche Gruppe beschreiben allerdings Jaeger und Mourant (2001). Als Ursache eines Geschlechtereinflusses wurden Unterschiede in der körperlichen Aktivität, der Exposition (nauseogene Situationen) sowie des Antwortverhaltens (engl. reporting bias) untersucht: hier konnten keine signifikanten Unterschiede demonstriert werden (Dobie et al. 2001). Es gibt jedoch Hinweise, dass hormonelle Einflüsse eine Rolle bei der Empfindlichkeit gegenüber Kinetosen spielen. Einerseits existiert ein Einfluss des 
Menstruationszyklus auf Simulator-Sickness-Symptome, andererseits zeigen postmenopausale Frauen keine signifikanten Unterschiede zu altersentsprechenden Männern (Clemens und Howarth 2001).

In unserer Studie ließ sich anhand des Simulator Sickness Questionnaire kein Geschlechtseffekt im Sinne einer höheren Empfindlichkeit von Frauen zeigen: diese hätten sonst einen signifikant stärkeren Anstieg im Testverlauf zeigen müssen. Eher ist zu vermuten, dass Körperwahrnehmung und Antwortverhalten eine Rolle spielen könnten (s.a. Jokerst et al. 1999.)

Der von Kolasinski $(1995,1996)$ in seinen Übersichtsarbeiten beschriebene Effekt des Alters auf Simulator-Sickness-Symptome, wonach jüngere Probanden eher zur Simulator Sickness neigen, konnte in unserer Studie statistisch nicht nachgewiesen werden. Dies könnte zum einen an der Altersverteilung unserer Stichprobe mit einem Mittelwert von 47.8 Jahren liegen (Min. 15, Max. 76). Zum anderen stammen die Daten zur Altersabhängigkeit nicht aus VirtualReality-Anwendungen, sondern aus der Motion Sickness-Forschung. Inwiefern diese Ergebnisse, die z.B. die Kinetosenanfälligkeit beim Autofahren beschreiben, übertragbar sind, ist unklar. Als Indiz, dass auch jüngere Anwendergruppen keine hohe Anfälligkeit für Simulator Sickness besitzen, ist eine Anwendung zur neuropsychologischen Testung und kognitiven Rehabilitation von Kindern zu nennen (Rizzo et al. 2002): hier wurden ,keine' der per SSQ erfassten Nebenwirkungen registriert.

Ein Einfluss der Gruppenzugehörigkeit (gesunde Teilnehmer, Patientengruppen) auf die SSQ. Scores konnte in unserer Studie nicht gezeigt werden. Dies impliziert nicht, dass generell für neurologische Erkrankungen ein unbedenklicher Einsatz der VR-Technik möglich ist, da als Ausschlusskriterium der klinischen Studie z.B. Übelkeit und Schwindel galten. Ob Patienten mit diesen Charakteristika eventuell eine deutlich höhere Anfälligkeit für SSQ-Symptome besitzen, ist mit dieser Studie nicht beantwortet worden. Weiterhin fehlen Daten zu großen Teilen des psychiatrischen Patientenguts mit Wahrnehmungsstörungen (z.B. Schizophrenien). Weiterführende statistische Untersuchungen zum Einfluss neurologischer und psychiatrischer Erkrankungen auf das Auftreten von Simulator-Sickness-Symptomen sind bislang nicht veröffentlicht worden. Als Beispiel einer ähnlich starken Anfälligkeit für Nebenwirkungen kann eine Studie mit 34 Patienten nach unilateraler temporaler Lobektomie und 21 Kontrollprobanden zum topographischen Lernen mittels Monitorkonfiguration genannt werden. Hier brachen wegen Übelkeit 2 der Patienten und 3 der Probanden ab (Spiers et al. 2001).

Während in unserer Studie die Anzeigemodalität als einziger wesentlicher nauseogener Faktor nachgewiesen wurde, zeigen die Ergebnisse anderer Arbeitsgruppen, dass die Modifikation verschiedener Parameter einer VR-Anwendung ebenfalls deutlichen Einfluss haben. Bis auf den Testaufgabeninhalt (Park, Labyrinth) und die mit der Anzeigemodalität verbundenen Unterschiede wurden in unserer Studie Parameter wie Freiheitsgrade bei der Navigation 
(Stanney und Hash 1998) oder Geschwindigkeit (So et al. 2001) konstant gehalten und im vorhinein auf eine niedrige Inzidenz an Nebenwirkungen ausgerichtet.

Die Daten der PILOT-Studie sind aufgrund des Versuchablaufs nur eingeschränkt bewertbar: inwiefern die HMD-Nebenwirkungen im Sinne eines Summations- oder Schwelleneffekts teilweise durch die Projektionsdarbietung hervorgerufen wurden (s. Abb. 2.4.), ist nicht eindeutig zu klären. Wesentlicher Kritikpunkt an der KLIN-Studie hinsichtlich des Einflusses der Anzeigemodalität ist die fehlende systematische Randomisierung von Teilnehmergruppen unter Berücksichtigung von Alter und Geschlecht zu den drei Anzeigemodalitäten.

Inwieweit das Ziel der Arbeit, im Rahmen der Systementwicklung eine Verringerung und Optimierung von Nebenwirkungen zu bewirken, erreicht wurde, ist aufgrund der unterschiedlichen Anzahl der Testteilnehmer nicht sicher zu bewerten (PILOT-Studie: 22 Probanden; KLIN-Studie 215 Teilnehmer). In der PILOT-Studie kam es bei $4.8 \%$ der Probanden zu einem Testabbruch wegen Nebenwirkungen, in der KLIN-Studie bei $1.4 \%$ des Gesamtkollektivs. Bei Verwendung von Monitor und Projektion musste kein Teilnehmer wegen Nebenwirkungen abbrechen, was als sehr positives Ergebnis zu werten ist. Die Inzidenz von Testabbrechern wegen unerwünschter Nebenwirkungen wird in der Literatur bei VR. Anwendungen unterschiedlich angegeben, bei vergleichbaren Anwendungen wurden bis zu $16 \%$ Prozent Abbrecher genannt (Witmer et al. 1996).

Anhand der vorliegenden Daten wird das grundsätzliche Dilemma bezüglich der Notwendigkeit eines Head Mounted Displays als ,der' klassischen Virtual-Reality-Komponente deutlich: mit dem HMD wird eine geringfügig höhere Immersion bei den Testteilnehmern erreicht, unter Inkaufnahme von Nebenwirkungen, Anschaffungskosten und - v.a. mit dem visuell hochqualitativen aber schweren HMD-Modell der PILOT-Studie - einer Komforteinbuße. Über den Einsatz eines HMD sollte letztendlich unter Berücksichtigung der Vor- und Nachteile im Einzelfall entschieden werden (s.a. Johnson et al. 1998), eine generelle Notwendigkeit für neurowissenschaftliche Anwendungen wird nicht gesehen (Rizzo et al. 1998a).

Ist die Inzidenz von Nebenwirkungen in dieser Studie akzeptabel für einen klinischen Einsatz des Testsystems bei den getesteten Patientengruppen? - Die Inzidenz von 1.4\% Testabbrechern wäre durch die Verwendung ausschließlich nicht-immersiver Anzeigeverfahren (Monitor, Projektion) anhand unserer Datenlage weiter reduzierbar. Bei der Benutzung eines HMD wäre dagegen eine höhere Inzidenz zu erwarten, doch handelt es sich bei den beobachteten Nebenwirkungen um vorübergehende Symptome, die nach Entfernen des Stimulus rasch nachlassen. Lediglich in der Frühphase der Testentwicklung wurde einmalig eine heftige Reaktion mit Übelkeit beobachtet.

Für einen orientierenden Vergleich der Nebenwirkungsrate des VR-Testsystems kann als Beispiel eines anderen diagnostischen Verfahrens die Magnetresonanztomographie 
herangezogen werden. Das MRT, das für Fragestellungen struktureller zerebraler Schädigungen und Abbauprozesse sowie zunehmend zur Untersuchung funktioneller Systeme eingesetzt wird, zeigt Abbruchquoten wegen temporärer Nebenwirkungen und Klaustrophobie von $4.10 \%$, bis zu 30\% der Patienten erleben eine Angstreaktion bei der MR-Untersuchung (Münte et al. 2002). Dennoch wird, auch mit Kontrastmittelapplikation, das MRT generell als sichere Untersuchungsmethode eingestuft (Ordrige et al. 2000, Chung 2002). Unter Berücksichtigung der Daten der präklinischen und klinischen Evaluationsstudie kann die Inzidenz nennenswerter VR-Nebenwirkungen für die untersuchte Anwendung und das Patientenkollektiv als tolerabel eingestuft werden. 


\section{Zusammenfassung}

Hintergrund: Virtuelle Realität (VR) bietet in der Medizin und insbesondere in den Neurowissenschaften vielversprechende Anwendungsmöglichkeiten. So werden z.B. VRRealisationen zur Testung von visuell-räumlichem Lernen bei neuropsychologischen Fragestellungen den üblichen Paper- und Pencil-Tests als überlegen eingeordnet.

Hindernisse für eine rasche Verbreitung in der klinischen Anwendung sind u.a. der hohe Investitionsaufwand, unerwünschte Nebenwirkungen bei der Anwendung und die personal- und kostenintensive Entwicklung. Bei der Nutzung der VR-Technologie durch Patienten haben bisherige Arbeiten Fragen über Eignung und Notwendigkeit bestehender VR-Komponenten und über das Auftreten von Nebenwirkungen weitgehend offen gelassen.

Ziel: Mit der vorliegenden Arbeit wurde der Aufbau eines interdisziplinär nutzbaren VirtualReality-Labors am Universitätsklinikum Göttingen verfolgt, um klinische Gruppen mit personellen und technischen Ressourcen bei der Entwicklung VR-basierter Anwendungen zu unterstützen. Als Pilotprojekt sollte die Entwicklung sowie die präklinische und klinische Evaluation eines neuropsychologischen Testsystems zum visuell-räumlichen und topographischen Lernen durchgeführt werden. In diesem Rahmen sollten Fragestellungen zur Praktikabilität, Wirkungen und Nebenwirkungen virtueller Realität bearbeitet werden.

Methode: Zu Beginn der Konzeptionsphase des VR-Labors (1997/98) wurde eine Marktübersicht und technische Analyse bestehender medizinischer VR-Anwendungen durchgeführt. Auf Grundlage eines strukturierten Systemdesigns wurde ein neuropsychologisches Testsystem zum räumlichen Lernen entwickelt. Zur Evaluation wurde eine präklinische Pilotstudie mit 22 gesunden Probanden (PILOT-Studie) sowie eine klinische Studie (KLIN-Studie) mit 215 Teilnehmern durchgeführt (75 Gesunde, 40 Patienten vor epilepsiechirurgischem Eingriff, 25 Patienten nach epilepsiechirurgischem Eingriff, 27 Schlaganfallpatienten, 48 Patienten mit V.a. Alzheimer-Demenz). Nebenwirkungen der VRAnwendung wurden mit dem Simulator Sickness Questionnaire (SSQ) systematisch dokumentiert und es erfolgte eine schriftliche Nachbefragung hinsichtlich Praktikabilität und Wirkungen (Immersion, empfundener Realismus). Anhand varianz- und korrelationsanalytischer Verfahren wurden mögliche Einflussfaktoren auf Wirkungen und Nebenwirkungen untersucht.

Ergebnisse: Anhand der Analyse existierender VR-Anwendungen wurde ein PC-basiertes VRLabor mit der Möglichkeit von Desktop-basierten (Bildschirm), semi-immersiven (Projektion) und immersiven (Head Mounted Display, HMD) VR-Anwendungen eingerichtet. Das neuropsychologische Testsystem beinhaltete Aufgabenstellungen zur topographischen Orientierung, zu visuell-räumlichem Lernen und exekutiven Funktionen, operationalisiert durch einen naturalistischen Park und verschiedene Labyrinthumgebungen. Eine Testadministration und semiautomatische Testauswertung wurden datenbankbasiert umgesetzt. Anhand der 
Ergebnisse der PILOT-Studie wurden für die KLIN-Studie Aufgabenoperationalisierung, Visualisierung und Komponentenauswahl modifiziert. Hinsichtlich der Praktikabilität und Benutzerbewertung zeigte sich für die Anzeigemodalität (Projektionssystem vs. HMD) in der PILOT-Studie keine Benutzerpräferenz. Das HMD der PILOT-Studie (Virtual Research V8) erwies sich nur als bedingt alltagstauglich und wurde in der KLIN-Studie durch ein leichteres und komfortableres Modell ersetzt (Sony Glasstron). Trotz Zurückhaltung älterer Testteilnehmer in Bezug auf das HMD-Verfahren wurden insgesamt wenig Bedienungsprobleme beobachtet. Die Bewertung von Immersion und Realismus zeigte keine sicheren Vorteile einer Anzeigemodalität. Bedingt durch Nebenwirkungen wurden Testabbruchquoten zwischen 1.4\% (KLIN-Studie) und $4.8 \%$ (PILOT-Studie) verzeichnet. Im Testverlauf ließen sich für das Gesamtkollektiv lediglich in der KLIN-Studie im SSQ-Subscore, Disorientation' ein signifikanter Anstieg von Symptomen beobachten. Die weiterführende Analyse der Ergebnisse identifizierte als wesentlichen Risikofaktor für das Auftreten von Simulator-Sickness-Symptomen das Head Mounted Display. Im Vergleich zu diesem konnten für den Projektions- und Monitormodus kein verstärktes Auftreten von Simulator-Sickness-Symptomen beobachtet werden. Keinen signifikanten Einfluss auf das Entstehen unerwünschter Nebenwirkungen im Testverlauf zeigten das Geschlecht, das Alter sowie die Zugehörigkeit zu einer Teilnehmergruppe (Kontrollgruppe, Patienten), allerdings wurden bei weiblichen Testteilnehmern unabhängig vom Testverlauf höhere SSQ-Scores beobachtet.

Fazit: Die vorliegende Arbeit hat die Leistungsfähigkeit und Funktionalität des wissenschaftlichen Konzepts eines medizinischen VR-Labors bestätigt. In der Zwischenzeit konnten weitere Projekte mit Anbindung an das VR-Labor begonnen werden. Mit einem System, das auf PC-Basis aufgebaut wurde, konnte zu Projektbeginn eine wirtschaftliche Lösung für eine leistungsfähige Ausstattung gefunden werden. Dieses Konzept findet inzwischen breite Anwendung.

In dieser Arbeit wurde zum ersten Mal für eine klinisch-neurowissenschaftliche VR-Anwendung das Auftreten von Nebenwirkungen systematisch untersucht. Hierbei konnte die Sicherheit des vorliegenden Testsystems für Patienten bei nicht-immersiven Anzeigeverfahren demonstriert werden. Die Ergebnisse der vorliegenden Studie sind aufgrund der Vielfalt technischer VR. Komponenten und medizinischer VR-Anwendungen jedoch nicht generalisierbar. Inwiefern immersive Anzeigeverfahren (z.B. Head Mounted Displays) für neuropsychologische Anwendungen notwendig oder konventioneller Technik überlegen sind, kann anhand der Ergebnisse nicht beurteilt werden. Wesentliche Vorteile hinsichtlich Realismus und Immersion lassen sich nicht herausarbeiten, demgegenüber stehen ein schlechteres Handling, eine Zurückhaltung älterer Testteilnehmer gegenüber der Technik und eine stärkere Nauseogenität. Aus ethischen, rechtlichen und wirtschaftlichen Gründen sollte auch zukünftig vor der breiten Anwendung vor allem immersiver VR-Technologien mit Patienten eine Risikobewertung von Technik und konkreter Anwendung in kontrollierten Untersuchungen erfolgen. 


\section{Anhang}

\subsection{Verzeichnis der verwendeten Abkürzungen}

$\begin{array}{ll}\text { Abb. } & \text { Abbildung } \\ \text { AD } & \text { Patienten mit V.a. Alzheimer-Demenz } \\ \text { CT } & \text { Computertomographie } \\ \text { CTRL } & \text { gesunde Probanden der Kontrollgruppe } \\ \text { D-Score } & \text { Disorientation-Score } \\ \text { EEG } & \text { Elektroenzephalogramm } \\ \text { engl. } & \text { englisch } \\ \text { EPI-POST } & \text { Patienten nach einem epilepsiechirurgischen Eingriff } \\ \text { EPI-PRÄ } & \text { Patienten vor einem epilepsiechirurgischen Eingriff } \\ \text { fMRI } & \text { Funktionelle Magnetresonanztomographie } \\ \text { FOV } & \text { engl. Field of view, Gesichtsfeldbreite } \\ \text { HMD } & \text { Head Mounted Display; Datenhelm } \\ \text { KLIN-Studie } & \text { Klinische Evaluationsstudie } \\ \text { LCD } & \text { engl. Liquid Crystal Display, Flüssigkristallbildschirm } \\ \text { MB } & \text { Megabyte } \\ \text { MHz } & \text { Megahertz } \\ \text { MMSE } & \text { Mini-Mental State Examination } \\ \text { MRT } & \text { Magnetresonanztomographie } \\ \text { N-Score } & \text { Nausea-Score } \\ \text { O-Score } & \text { Oculomotor-Score } \\ \text { PC } & \text { Personal Computer } \\ \text { PET } & \text { Positronen-Emissions-Tomographie } \\ \text { PILOT-Studie } & \text { präklinische Evaluationsstudie } \\ \text { SOP } & \text { Sense of Presence } \\ \text { SSQ } & \text { Simulator Sickness Questionnaire } \\ \text { STROKE } & \text { Schlaganfallpatienten } \\ \text { T-Score } & \text { Total-Score } \\ \text { VR } & \end{array}$




\subsection{Simulator Sickness Questionnaire}

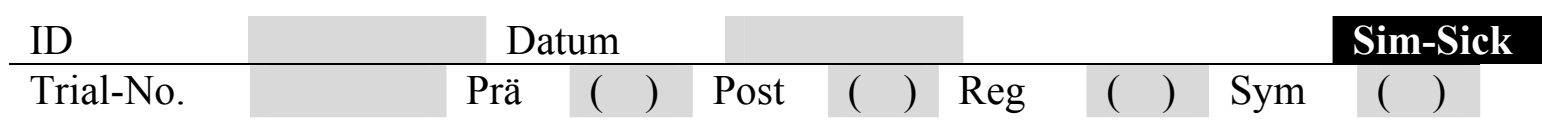

\section{Fragebogen}

Bitte füllen Sie folgenden Fragebogen aus, der verschiedene Symptome aufführt, die bei der Benutzung von virtuellen Umgebungen auftreten können. Die Daten werden anonymisiert, d.h. ohne eine mögliche Zuordnung zu Ihrer Person, gespeichert. Falls Sie Fragen zu den einzelnen Symptomen haben, sprechen Sie bitte Ihren Testleiter an.

\section{Symptom Ausprägung}

\begin{tabular}{|c|c|c|c|c|}
\hline (1) Allgemeines Unwohlsein & $\begin{array}{l}\text { ( ) } \\
\text { nicht vorhanden }\end{array}$ & $\begin{array}{l}(\text { ) } \\
\text { etwas }\end{array}$ & $\begin{array}{l}(\mathrm{r}) \\
\text { deutlich }\end{array}$ & $\begin{array}{l}(\text { ) }) \\
\text { sehr stark }\end{array}$ \\
\hline (2) Müdigkeit & $\begin{array}{l}\text { ( ) } \\
\text { nicht vorhanden }\end{array}$ & $\begin{array}{l}(\text { ) } \\
\text { etwas }\end{array}$ & $\begin{array}{l}(\text { ) } \\
\text { deutlich }\end{array}$ & $\begin{array}{l}(\text { ) }) \\
\text { sehr stark }\end{array}$ \\
\hline (3) Kopfschmerzen & $\begin{array}{l}\text { ( ) } \\
\text { nicht vorhanden }\end{array}$ & ( ) & $\begin{array}{l}\text { ( ) } \\
\text { deutlich }\end{array}$ & $\begin{array}{l}\text { ( ) } \\
\text { sehr stark }\end{array}$ \\
\hline (4) Überanstrengte Augen & $\begin{array}{l}\text { ( ) } \\
\text { nicht vorhanden }\end{array}$ & ( ) & $\begin{array}{l}\text { ( ) } \\
\text { deutlich }\end{array}$ & $\begin{array}{l}\text { ( ) } \\
\text { sehr stark }\end{array}$ \\
\hline (5) Schwierigkeiten beim Scharfsehen & $\begin{array}{l}\text { ( ) } \\
\text { nicht vorhanden }\end{array}$ & $\begin{array}{l}\text { ( ) } \\
\text { etwas }\end{array}$ & ( ) & $\begin{array}{l}\text { ( ) } \\
\text { sehr stark }\end{array}$ \\
\hline (6) Erhöhter Speichelfluß & $\begin{array}{l}\text { ( ) } \\
\text { nicht vorhanden }\end{array}$ & ( ) & $\begin{array}{l}\text { ( ) } \\
\text { deutlich }\end{array}$ & $\begin{array}{l}\text { ( ) } \\
\text { sehr stark }\end{array}$ \\
\hline (7) Schwitzen & $\begin{array}{l}\text { ( ) } \\
\text { nicht vorhanden }\end{array}$ & $\begin{array}{l}\text { ( ) } \\
\text { etwas }\end{array}$ & $\begin{array}{l}\text { ( ) } \\
\text { deutlich }\end{array}$ & $\begin{array}{l}\text { ( ) } \\
\text { sehr stark }\end{array}$ \\
\hline (8) Übelkeit & $\begin{array}{l}\text { ( ) } \\
\text { nicht vorhanden }\end{array}$ & $\begin{array}{l}\text { ( ) } \\
\text { etwas }\end{array}$ & $\begin{array}{l}\text { ( ) } \\
\text { deutlich }\end{array}$ & $\begin{array}{l}\text { ( ) } \\
\text { sehr stark }\end{array}$ \\
\hline (9) Konzentrationsschwierigkeiten & $\begin{array}{l}\text { ( ) } \\
\text { nicht vorhanden }\end{array}$ & $\begin{array}{l}(\text { ) } \\
\text { etwas }\end{array}$ & $\begin{array}{l}\text { ( ) } \\
\text { deutlich }\end{array}$ & $\begin{array}{l}\text { ( ) } \\
\text { sehr stark }\end{array}$ \\
\hline (10) Druckgefühl im Kopf & $\begin{array}{l}\text { ( ) } \\
\text { nicht vorhanden }\end{array}$ & $\begin{array}{l}\text { ( ) } \\
\text { etwas }\end{array}$ & $\begin{array}{l}\text { ( ) } \\
\text { deutlich }\end{array}$ & $\begin{array}{l}\text { ( ) } \\
\text { sehr stark }\end{array}$ \\
\hline (11) Getrübtes Sehen & $\begin{array}{l}\text { ( ) } \\
\text { nicht vorhanden }\end{array}$ & ( ) & $\begin{array}{l}\text { ( ) } \\
\text { deutlich }\end{array}$ & $\begin{array}{l}\text { ( ) } \\
\text { sehr stark }\end{array}$ \\
\hline (12) Schwindel bei geöffneten Augen & $\begin{array}{l}\text { ( ) } \\
\text { nicht vorhanden }\end{array}$ & $\begin{array}{l}\text { ( ) } \\
\text { etwas }\end{array}$ & () ) & $\begin{array}{l}\text { ( ) } \\
\text { sehr stark }\end{array}$ \\
\hline $\begin{array}{l}\text { (13) Schwindel bei geschlossenen } \\
\text { Augen }\end{array}$ & ( ) & ( ) & ( ) & $\underset{\text { sehr stark }}{(\text { ) }}$ \\
\hline (14) Drehschwindel & $\begin{array}{l}\text { ( ) } \\
\text { nicht vorhanden }\end{array}$ & $\begin{array}{l}\text { ( ) } \\
\text { etwas }\end{array}$ & $\begin{array}{l}\text { ( ) } \\
\text { deutlich }\end{array}$ & $\begin{array}{l}\text { ( ) } \\
\text { sehr stark }\end{array}$ \\
\hline (15) Wahrnehmung des Magens & $\begin{array}{l}\text { ( ) } \\
\text { nicht vorhanden }\end{array}$ & $\begin{array}{l}\text { ( ) } \\
\text { etwas }\end{array}$ & $\begin{array}{l}\text { ( ) } \\
\text { deutlich }\end{array}$ & $\begin{array}{l}\text { ( ) }) \\
\text { sehr stark }\end{array}$ \\
\hline (16) Aufstoßen & $\begin{array}{l}\text { ( ) } \\
\text { nicht vorhanden }\end{array}$ & ( ) & $\begin{array}{l}\text { ( ) } \\
\text { deutlich }\end{array}$ & $\begin{array}{l}\text { ( ) } \\
\text { sehr stark }\end{array}$ \\
\hline
\end{tabular}




\subsection{Berechnung der Scores für den Simulator Sickness Questionnaire}

Die SSQ-Scores werden auf der Grundlage der 16 Einzelantworten gebildet, wobei für jede Antwort 0 (keine Beschwerden) bis 3 (sehr starke Beschwerden) Punkte gewertet werden. Die SSQ-Scores (N - Nausea, O - Oculomotor, D - Disorientation, T - Total) setzen sich wie folgt zusammen:

$[1]=$ Summe der Antworten 1, 6, 7, 8, 9, 15, 16

[2] = Summe der Antworten 1, 2, 3, 4, 5, 9, 11

[3] = Summe der Antworten 5, 8, 10, 11, 12, 13, 14

$N=[1] \times 9,54$

$\mathrm{O}=[2] \times 7,58$

$\mathrm{D}=[3] \times 13,92$

$T=([1]+[2]+[3]) \times 3,74$

Anmerkung: die Originalarbeit zur Entwicklung des Simulator Sickness Questionnaire ist in englischer Sprache abgefasst (Kennedy u. Lane 1993). Die Übersetzung des Fragebogens in die deutsche Sprache wurde durch Mitarbeiter der Abteilung Psychiatrie / Schwerpunkt Neuropsychologie vorgenommen. 


\subsection{Präklinische Studie: Prä-Test Fragebogen}

ID

Datum

Prä-Test

\section{Fragebogen}

Bitte füllen Sie folgenden Fragebogen aus, in dem wir Sie um einige persönliche Angaben bitten. Die Daten werden anonymisiert, d.h. ohne eine mögliche Zuordnung zu Ihrer Person, ausgewertet. Falls Sie Fragen zu den einzelnen Punkten haben, sprechen Sie bitte Ihren Testleiter an.
(1) Geschlecht
( ) männlich
( ) weiblich

(2) Geburtsdatum

(3) Abgeschlossene

Schulbildung
Art der Schule:
( ) Sonderschule
( ) Hauptschule
( ) Realschule
( ) Gymnasium
( ) Fach- oberschule
( ) $\mathrm{FH} /$ Hochschule

Dauer: Jahre
(4) Beruf
( ) Freiberufler
( ) Arbeiter
( ) Landwirt
( ) leitender
Angestellter
( ) Hausfrau
( ) ungelernter
( ) Student/
Arbeiter
( ) Angestellter
Schüler
( )

(5) Sind bei Ihnen oder in Ihrer Familie psychiatrische oder (6.1)

( ) nein, keine neurologische Erkrankungen Bei mir selbst: ( ) ja, Art der Erkrankung: bekannt? ( ) nein, keine

In der Familie: ( ) ja, Art der Erkrankung: 
(6) Nehmen Sie regelmäßig Medikamente ein?
( ) nein

( ) ja Präparate, Dosis:

(7.1) Haben Sie Sehprobleme?

( ) ja

( ) nein

(7.2) Wenn ja, benutzen Sie ..

( ) eine Brille

( ) Kontaktlinsen

(7.3) Wenn ja, sind Sie ...

( ) weitsichtig

( ) kurzsichtig

( )

(8) Wie würden Sie Ihre Kenntnisse zur Benutzung von ( ) 1 sehr gut
( ) 2

( ) 3

( ) 4

( ) 5

( ) 6

Computern einschätzen?

(9) Wie häufig spielen Sie Computerspiele?

(10) Sind jemals bei Ihnen während der Benutzung von Computer-/ Videospielen körperliche oder seelische Probleme aufgetreten?

$\begin{array}{ll}\text { ( ) } 1 & \text { ( ) } 2 \\ \text { sehr oft }\end{array}$

( ) 3 sehr oft
( ) nein

( ) ja, körperliche Probleme.
Art der Probleme:
( ) 4
( ) 5
( ) 6
nie 


\subsection{Präklinische Studie: Post-Test Fragebogen}

ID

Datum

Post-Test

\section{Fragebogen}

Bitte füllen Sie folgenden Fragebogen aus, der verschiedene Punkte aufführt, die bei der Bewertung und Weiterentwicklung des Testsystems hilfreich sein können. Die Daten werden anonymisiert, d.h. ohne eine mögliche Zuordnung zu Ihrer Person, ausgewertet. Falls Sie Fragen zu den einzelnen Punkten haben, sprechen Sie bitte Ihren Testleiter/ Testleiterin an.

(1) Wie haben Sie den

Schwierigkeitsgrad für die

( ) 1

( ) 2

( ) 3

( ) 4

( ) 5

( ) 6

Aufgabenstellung empfunden?

leicht

sehr

schwer

(2.1) Sind Sie mit der

Fortbewegung durch die Tastatur

( ) ja

( ) nein

zurechtgekommen?

(2.2) Wenn nein, was hat Ihnen

Schwierigkeiten gemacht?

(2.3) Sind Sie mit der

( ) ja

( ) nein

Fortbewegung mit Datenhelm und

Maus zurechtgekommen?

(2.4) Wenn nein, was hat Ihnen

Schwierigkeiten gemacht?

(3) Wie realistisch erschienen

Ihnen die virtuellen Umgebungen?

(3.1) mit dem Datenhelm:

( )

( ) 2

( ) 3

( ) 4

( ) 5

( ) 6

sehr

( ) 2

( ) 3

( ) 4

( ) 5

( ) 6

sehr

wenig 
(4) Wie realistisch konnten Sie sich in der virtuellen Umgebung umsehen?

(4.1) mit dem Datenhelm:

( )

sehr

( ) 2

( ) 3

( ) 4

( ) 5

( ) 6

wenig

(4.2) mit der Projektionsleinwand:

$\begin{array}{llllll}\text { ( ) } 1 & \text { ( ) } 2 & \text { ( ) } 3 & \text { ( ) } 4 & \text { ( ) } 5 & \text { ( ) } 6 \\ \text { sehr } & & & & & \text { wenig }\end{array}$

(5) Wie stark haben Sie sich in die virtuelle Umgebung hineinversetzt gefühlt?
(5.1) mit dem Datenhelm:
( ) 1
( ) 2
( ) 3
( ) 4
( ) 5
( ) 6
sehr
wenig
(5.2) mit der Projektionsleinwand:
( ) 1
( ) 2
( ) 3
( ) 4
( ) 5
( ) 6
sehr
wenig

(6) Wie stark haben Sie während der Versuche Eindrücke ausserhalb der virtuellen Umgebung (Raum, Personen) wahrgenommen?
(6.1) mit dem Datenhelm:
( ) 1
( ) 2
( ) 3
( ) 4
( ) 5
( ) 6
( ) 2
( ) 3
( ) 4
( ) 5
( ) 6
sehr
wenig

(7) Haben Sie die Benutzung des

Datenhelms als beklemmend
( ) ja
( ) nein empfunden?

(8) Welche Art der Anzeige würden Sie bevorzugen?
( ) Projektions- ( ) Datenhelm leinwand
(9) Wie würden Sie die Erfahrung mit der Benutzung des Testsystem beschreiben?
( ) interessant
( ) langweilig
( )
( ) motivierend ( ) frustrierend 
(10.1) Sind Ihnen persönliche Strategien bei der Bearbeitung der

Aufgaben aufgefallen?

(10.2) Falls ja, können Sie die

Strategien beschreiben?
( ) ja
( ) nein
(11) Waren Ihnen der Testablauf
( ) ja
( ) nein und die gestellten Fragen verständlich?

(12) Freie Anmerkungen: 


\subsection{Klinische Studie: Nachbefragung}

VR-Projekt: NACHBEFRAGUNG Vp-Nr:___ Datum:

1. Ich empfand die Aufgabe insgesamt...

im Park: $\quad$ sehr schwer $1-2-3-4-5$ sehr leicht

im Labyrinth: $\quad$ sehr schwer $1-2-3-4-5$ sehr leicht

im Nebel: $\quad$ sehr schwer $1-2-3-4-5$ sehr leicht

2. Um den Park beim ersten Kennenlernen sinnvoll durchsuchen zu können,

habe ich folgende Strategie benutzt:

0 gar keine

0 ich habe immer möglichst sofort eine Sackgasse durchsucht, wenn ich an eine Gabelung kam

0 ich habe immer zunächst die rechte (bzw. linke) Abzweigung gewählt

0 ich habe das Betreten von Sackgassen vermieden

0 andere Strategie, und zwar:

3. Um das Ziel im zweiten (dritten usw.) Durchgang schnell wiederzufinden, habe ich mir im Park folgendes eingeprägt:

0 eine Abfolge von Richtungswechseln (z.B. rechts, links, links,...)

0 eine Abfolge von markanten Orten und Richtungswechseln (z.B. am Spielplatz links,

vor der Brücke rechts, am Backsteinhaus rechts,...)

0 einen räumlichen Überblick über die Landschaft oder über die Form des Weges

0 etwas anderes:

4. Um das Labyrinth beim ersten Kennenlernen sinnvoll durchsuchen zu können,

habe ich folgende Strategie benutzt:

0 gar keine

0 ich habe immer möglichst sofort eine Sackgasse durchsucht, wenn ich an eine Gabelung kam

0 ich habe immer zunächst die rechte (bzw. linke) Abzweigung gewählt

0 ich habe das Betreten von Sackgassen vermieden

0 andere Strategie, und zwar:

5. Um das Ziel im zweiten (dritten usw.) Durchgang schnell wiederzufinden, habe ich mir im Labyrinth folgendes eingeprägt:

0 eine Abfolge von Bewegungs-Anweisungen (z.B. rechts, links, links,...)

0 einen räumlichen Überblick über das Labyrinth oder über die Form des Weges

0 etwas anderes: 
6. Bei der Fortbewegung bin ich mit Datenhelm und Maus zurechtgekommen.

0 ja 0 nein, ich hatte Schwierigkeiten mit

7. Die Benutzung des Datenhelms war für mich unangenehm / beängstigend.

0 ja 0 nein

8. Die Erklärung des Testablaufs, die Anweisungen und die Fragen waren verständlich. 0 ja 0 nein, mir war folgendes unklar:

9. Die virtuellen Umgebungen erschienen mir realistisch.

Park: $\quad$ wenig realistisch $01-02-03-04-05$ sehr realistisch

Labyrinth: wenig realistisch $01-02-03-04-05 \mathrm{sehr}$ realistisch

Nebel: $\quad$ wenig realistisch $01-02-03-04-05 \mathrm{sehr}$ realistisch

10. Ich habe mich in die Umgebungen hineinversetzt gefühlt.

Park: $\quad$ kaum $01-02-03-04-05$ sehr stark

Labyrinth: kaum $01-02-03-04-05$ sehr stark

Nebel: $\quad$ kaum $01-02-03-04-05$ sehr stark

11. Ich habe während des Experiments Eindrücke außerhalb der virtuellen Umgebungen wahrgenommen (z.B. Testleiter, andere Personen, Raum).

kaum $01 \longleftarrow 02-03-04-05$ sehr stark

12. Insgesamt war die Erfahrung mit dem Testsystem... (z.B. langweilig, interessant, frustrierend) im Park:

im Labyrinth:

im Nebel:

13. Ich hatte schon Vorerfahrungen mit dem Computer

0 gar keine

0 vereinzelte Erfahrungen / Grundkenntnisse (z.B. in Textverarbeitung)

0 häufige Erfahrungen / gute Kenntnisse (z.B. Grafikverarb., Computerspiele, Programmieren)

14. Im Alltag ist mein Orientierungsvermögen ...

sehr schlecht $01-02-03-04-05$ sehr gut 


\title{
6.7. Erläuterungen für gesunde Kontrollprobanden (Beispiel aus präklinischer Studie)
}

\author{
Erläuterungen für gesunde Kontrollprobanden
}

der Untersuchung "Virtual Reality-gestütztes neuropsychologisches Testsystem zur Diagnose und Therapie neuropsychiatrischer Erkrankungen"

Es handelt sich um eine wissenschaftliche Untersuchung, bei der die Fähigkeit, sich in einer fremden Umgebung zu orientieren, überprüft wird. Sie persönlich haben keinerlei Nutzen aus der Teilnahme an der Studie zu erwarten.

Die Aufgaben werden statt mit einem herkömmlichen Computer-Bildschirm mittels einer Datenbrille oder eines Wandprojektors dargestellt. Der Versuchsleiter wird Ihnen die Geräte zum Verständnis erklären und vorführen. Mit Hilfe dieser Geräte werden Sie in einer sogenannten virtuellen Umgebung Orientierungsaufgaben lösen. Eine virtuelle Umgebung ist dadurch charakterisiert, daß Sie in eine künstliche, nicht reale Umgebung versetzt werden, in der jedoch verschiedene Merkmale der Realität von einem Computer geschaffen werden.

In seltenen Fällen kann es durch die Datenbrille oder den Projektor in der virtuellen Umgebung zum Auftreten von Übelkeit, Kopfschmerzen oder Doppelbildern kommen. In diesem Fall sollten Sie den Versuch sofort abbrechen. Auch wenn Sie sich unsicher oder unwohl fühlen, sollten Sie den Versuch sofort beenden und dem Versuchsleiter Ihre Probleme oder Schwierigkeiten mitteilen.

Die Untersuchung dauert insgesamt ca. 90 Minuten. Sie können unabhängig von den vorgesehenen Pausen, falls Sie es wünschen, eine Pause einlegen. Falls Sie während der Untersuchung zu erschöpft sind, kann die Untersuchung an einem anderen Tag fortgesetzt werden.

Sie haben weiterhin jederzeit das Recht, ohne Angaben von Gründen die Untersuchung abzubrechen, ohne daß Ihnen daraus irgendwelche Nachteile entstehen.

Alle personenbezogenen Daten werden gemäß den geltenden rechtlichen Bestimmungen (Paragraph 203 StGB) vor unbefugter Offenbarung strikt geschützt. Die Daten werden nur anonym, ohne die Zuordnung der individuellen Ergebnisse zu einzelnen Personen, ausgewertet.

Prof. Dr. O. Rienhoff

(Abteilung Medizinische Informatik)

Dipl.-Psych. Arzt G.Weniger

(Abteilung Psychiatrie,

Schwerpunkt Neuropsychologie) 


\subsection{Erläuterung für Patienten (Beispiel aus klinischer Studie)}

\section{Erläuterung der Untersuchung „VR-gestütztes neuropsychologisches Testsystem zur Diagnose und Therapie neuropsychiatrischer Erkrankungen" (Patienten mit der Verdachtsdiagnose einer Demenz)}

Es handelt sich um eine wissenschaftliche Untersuchung, bei der die Fähigkeit, sich in einer fremden Umgebung zu orientieren, überprüft wird.

Die Untersuchungen werden zu zwei verschiedenen Zeitpunkten durchgeführt, wobei die zweite Untersuchung sechs Monate nach der ersten Testung durchgeführt wird.

In beiden Untersuchungen werden die Aufgaben zum einen direkt am Bildschirm eines Computers und zum anderen mittels einer sogenannten virtuellen Umgebung dargeboten. In seltenen Fällen kann es in der virtuellen Umgebung zum Auftreten von Übelkeit, Kopfschmerzen oder Doppelbildern kommen. In diesem Fall sollten Sie den Versuch sofort abbrechen. Auch wenn Sie sich unwohl oder unsicher fühlen, sollten Sie den Versuch sofort beenden, und dem Versuchsleiter ihre Probleme oder Schwierigkeiten mitteilen. Die Untersuchung dauert insgesamt circa 4 1 1/2 Stunden. Nach jeweils spätestens 1 1 1/2 Stunden wird eine Pause von 20 Minuten eingelegt. Sie können jedoch unabhängig von den vorgesehenen Pausen, falls Sie es wünschen, eine Pause einlegen. Falls Sie während der Untersuchung zu erschöpft sind, kann die Untersuchung auch an einem anderen Tag fortgesetzt werden.

Sie haben weiterhin jederzeit das Recht, ohne Angaben von Gründen die Untersuchung abzubrechen, ohne daß Ihnen daraus irgendwelche Nachteile hinsichtlich Ihrer Betreuung und Behandlung entstehen werden.

Alle personenbezogenen Daten werden gemäß den geltenden rechtlichen Bestimmungen (Paragraph 203 StGB) vor unbefugter Offenbarung strikt geschützt. Die Daten werden nur anonym, ohne die Möglichkeit der Zuordnung der individuellen Ergebnisse zu den einzelnen Personen, ausgewertet.

Prof. Dr. O. Rienhoff

(Abteilung Medizinische Informatik)

Dipl.-Psych. Arzt G.Weniger

(Abteilung Psychiatrie, Schwerpunkt Neuropsychologie)
Prof. Dr. E. Irle

(Schwerpunkt Neuropsychologie) 


\subsection{Einverständniserklärung für gesunde Kontrollprobanden (Beispiel aus präklinischer Studie)}

Einverständniserklärung für gesunde Kontrollprobanden

der Untersuchung „,Virtual Reality-gestütztes neuropsychologisches Testsystem zur Diagnose und Therapie neuropsychiatrischer Erkrankungen"

Ich habe die Erläuterungen der Untersuchung gelesen und erkläre mich bereit, an der Untersuchung teilzunehmen.

Ich habe jederzeit das Recht, ohne weitere Angabe von Gründen, die Untersuchung abzubrechen, ohne daß mir daraus irgendwelche Nachteile entstehen.

Alle von mir erhobenen Daten werden gemäß den rechtlichen Bestimmungen (Paragraph 203 StGB) vor unbefugter Offenbarung strikt geschützt. Weiterhin werden alle meine Daten nur anonym, ohne die Möglichkeit der Zuordnung meiner individuellen Ergebnisse zu meiner Person, ausgewertet.

Göttingen, den

Unterschrift des Probanden 


\subsection{Anleitung zur Testdurchführung (Beispiel der Labyrinth-Version aus präklinischer Studie)}

Sehr geehrter Versuchsteilnehmer, sehr geehrte Versuchteilnehmerin,

Vielen Dank für Ihre Teilnahme an unserem Versuch.

In diesem Versuch soll getestet werden, wie Sie Sich in einer Ihnen fremden Umgebung orientieren und welche Auswirkungen verschiedene Computer-Anzeigegeräte auf Ihr Testverhalten haben.

Dazu werden Ihnen verschiedene virtuelle, computergenerierte Labyrinthe dargeboten. Ziel dabei ist es, daß Sie in dieser Umgebung den Weg zu einem verborgenen Ziel-Zeichen finden. In den folgenden Durchläufen sollen Sie sich den kürzesten Weg dorthin merken und wiedererkennen, d.h. ohne Umwege zu gehen oder die falsche Abzweigung zu nehmen.

Im ersten Teil des Versuches verwenden wir ein Projektions-System zur Anzeige. In der virtuellen Umgebung können Sie mit Hilfe von Tasten auf der Computertastatur herumlaufen.

Im zweiten Teil des Versuches verwenden wir den Datenhelm zur Anzeige. In der virtuellen Umgebung können Sie mit Hilfe Ihrer Kopfbewegung und einer Maus sich fortbewegen.

Sowohl im ersten wie im zweiten Teil des Versuchs üben wir mit Ihnen zunächst das Umherlaufen, bis Sie sich sicher darin fühlen.

Beide Versuchsteile enthalten je einen Trainingsdurchgang und je fünf identisch gestaltete Versuchsdurchgänge.

Bei Fragen hilft Ihnen gerne die Versuchsleiterin / der Versuchleiter. 


\section{Übungsphase Labyrinth: Projektionssystem}

In den nächsten sechs Minuten haben Sie jetzt Gelegenheit, sich in einer Übungsumgebung an die Testsituation zu gewöhnen. Die Übungsumgebung ist ganz einfach gestaltet und soll Ihnen helfen zu lernen, wie Sie sich in der Testsituation bewegen und das Ziel finden können.

Vom Startpunkt aus können Sie sich auf den Wegen und innerhalb der Wände eines Labyrinths fortbewegen. Dabei können Sie sich drehen und von Weggabelung zu Weggabelung laufen, indem die Steuerungstasten der Tastatur gedrückt werden.

Noch einmal zur Erinnerung:

Die drei Tasten $\leftarrow, \rightarrow$ und $\uparrow$ ermöglichen die Fortbewegung:

$\rightarrow$-Taste: nach rechts drehen.

$\leftarrow$-Taste: nach links drehen.

个-Taste: Laufen des Weges bis zur nächsten Abzweigung.

Das Laufen zur nächsten Weggabelung funktioniert nur, wenn sich ein Weg in der Mitte Ihres Blickfeldes, also geradeaus, befindet.

Sie erkennen an einem Schild, daß Sie das Ziel erreicht haben. Dieses Schild ist von der Wegkreuzung aus nicht sichtbar, sondern erscheint erst, wenn Sie dem Ziel sehr nahe gekommen sind. Das Ziel befindet sich am Ende eines Weges.

Alle weiteren Fragen beantworten Ihnen die Versuchsleiterin/der Versuchsleiter gerne.

Wenn Sie das Gefühl haben, daß Sie sich sicher durch das Labyrinth bewegen können oder wenn die Übungszeit vorbei ist, beendet die Versuchleiterin/der Versuchsleiter die Übungsphase.

Haben Sie noch irgendwelche Fragen?

Wenn nicht, geht es jetzt weiter. 


\section{Versuchsteil Labyrinth: Projektionssystem}

Im nun folgenden Teil des Versuchs sollen Sie den Weg vom Start zum Ziel durch ein Labyrinth finden und in weiteren Durchläufen immer besser behalten.

Insgesamt gibt es für Sie fünf Durchläufe durch das Labyrinth, auch dann, wenn Sie nach einer geringeren Anzahl von Durchläufen bereits den Weg zum Ziel kennen sollten. Begonnen wird jedes Mal vom Startpunkt. Ein Durchlauf ist zu Ende, wenn Sie das Zielschild erblickt haben. Das Ziel befindet sich am Ende eines Weges. Es gibt nur einen möglichen Weg vom Start zum Ziel. Für jeden Durchgang haben Sie maximal fünf Minuten Zeit.

Versuchen Sie bitte, den Weg zum Ziel so direkt wie möglich zu wählen.

Jedesmal, wenn Sie das Zielschild erreicht haben, beendet die Versuchsleiterin/ der Versuchsleiter den Durchlauf und sorgt dafür, daß Sie in einem neuen Durchlauf wieder vom Startpunkt aus beginnen können.

Haben Sie noch Fragen? Dann geht es jetzt gleich los.

Noch einmal zu Ihrer Information:

$\rightarrow$-Taste: nach rechts drehen.

$\leftarrow$-Taste: nach links drehen.

$\uparrow$-Taste: Laufen des Weges bis zur nächsten Abzweigung.

Das Laufen zur nächsten Weggabelung funktioniert nur, wenn sich ein Weg in der Mitte Ihres Blickfeldes befindet. 


\section{Übungsphase Labyrinth: Datenhelm}

In den nächsten sechs Minuten haben Sie jetzt Gelegenheit, sich in einer Übungsumgebung an die Testsituation mit dem Datenhelm zu gewöhnen. Die Übungsumgebung ist ganz einfach gestaltet und soll Ihnen helfen zu lernen, wie Sie sich in der Testsituation bewegen und das Ziel finden können.

Vom Startpunkt aus können Sie sich auf den Wegen und innerhalb der Wände eines Labyrinths fortbewegen. Dabei können Sie sich mit Hilfe Ihrer Kopfbewegung nach links und rechts drehen und von Weggabelung zu Weggabelung laufen, indem Sie eine Taste auf der Maus drücken.

Noch einmal zur Erinnerung:

\section{Links-/Rechts-Kopfdrehung: Laufrichtung bestimmen Maustaste drücken: laufen bis zur nächsten Abzweigung.}

Am wohlsten fühlen Sie sich in der Regel bei der Benutzung des Datenhelms, wenn Sie nicht zu hastige Kopfbewegungen durchführen und vor dem Loslaufen Ihren Körper wie beim „normalen Laufen“ gerade richten.

Das Laufen zur nächsten Weggabelung funktioniert nur, wenn sich ein Weg in der Mitte Ihres Blickfeldes, also geradeaus, befindet.

Sie erkennen an einem Schild, daß Sie das Ziel erreicht haben. Dieses Schild ist von der Wegkreuzung aus nicht sichtbar, sondern erscheint erst, wenn Sie dem Ziel sehr nahe gekommen sind. Das Ziel befindet sich am Ende eines Weges.

Alle weiteren Fragen beantworten Ihnen die Versuchsleiterin/der Versuchsleiter gerne.

Wenn Sie das Gefühl haben, daß Sie sich sicher durch das Labyrinth bewegen können oder wenn die Übungszeit vorbei ist, beendet die Versuchleiterin/der Versuchsleiter die Übungsphase.

Haben Sie noch irgendwelche Fragen? Wenn nicht, geht es jetzt weiter. 


\section{Versuchsteil Labyrinth: Datenhelm}

Im nun folgenden Teil des Versuchs sollen Sie den Weg vom Start zum Ziel durch ein Labyrinth finden und in weiteren Durchläufen immer besser behalten.

Insgesamt gibt es für Sie fünf Durchläufe durch das Labyrinth, auch dann, wenn Sie nach einer geringeren Anzahl von Durchläufen bereits den Weg zum Ziel kennen sollten. Begonnen wird jedes Mal vom Startpunkt. Ein Durchlauf ist zu Ende, wenn Sie das Zielschild erblickt haben. Das Ziel befindet sich am Ende eines Weges. Es gibt nur einen möglichen Weg vom Start zum Ziel. Für jeden Durchgang haben Sie maximal fünf Minuten Zeit.

Versuchen Sie bitte, den Weg zum Ziel so direkt wie möglich zu wählen.

Jedesmal, wenn Sie das Zielschild erreicht haben, beendet die Versuchsleiterin/ der Versuchsleiter den Durchlauf und sorgt dafür, daß Sie in einem neuen Durchlauf wieder vom Startpunkt aus beginnen können.

Haben Sie noch Fragen? Dann geht es jetzt gleich los.

Noch einmal zur Erinnerung:

\section{Links-/Rechts-Kopfdrehung: Laufrichtung bestimmen Maustaste drücken: laufen bis zur nächsten Abzweigung.}

Am wohlsten fühlen Sie sich in der Regel bei der Benutzung des Datenhelms, wenn Sie nicht zu hastige Kopfbewegungen durchführen und vor dem Loslaufen Ihren Körper wie beim „normalen Laufen“ gerade richten.

Das Laufen zur nächsten Weggabelung funktioniert nur, wenn sich ein Weg in der Mitte Ihres Blickfeldes, also geradeaus, befindet. 


\subsection{Bildschirmmasken des Testadministrationsmoduls}

Abb. 6.1. Eingabe der Probandendaten

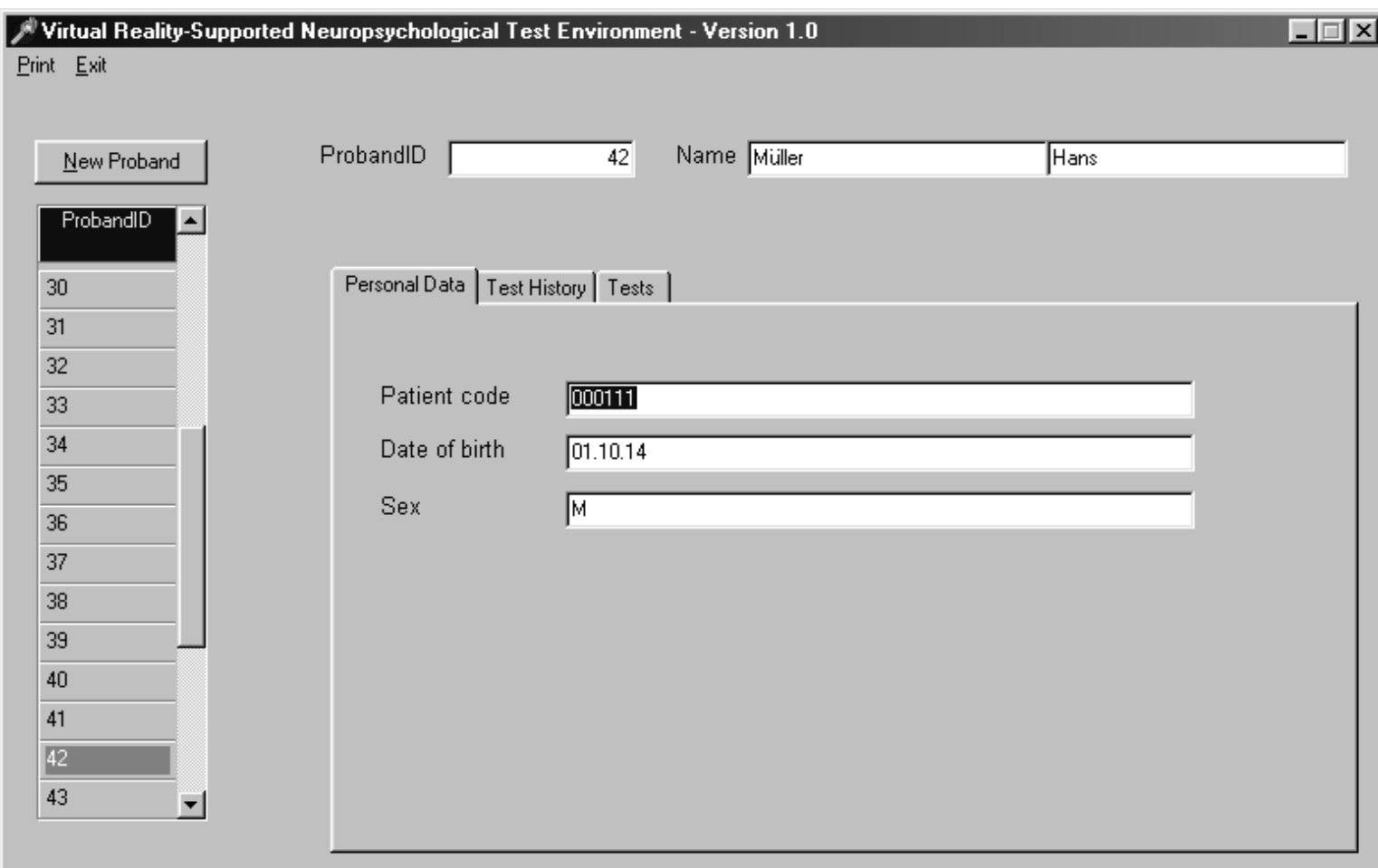

Abb. 6.2. Übersicht der durchgeführten VR-Tests eines Probanden

Virtual Reality-Supported Neuropsychological Test Environment - Version 1.0 Print Exit

\begin{tabular}{|c|c|c|c|c|c|c|}
\hline New Proband & ProbandID & 42 & \multirow[t]{2}{*}{ Name Miiller } & \multicolumn{2}{|l|}{ Hans } & \\
\hline \multicolumn{6}{|l|}{ ProbandID } & \\
\hline 30 & Personal Data & a Test History |Tests & & & & \\
\hline 31 & \multirow{2}{*}{$\begin{array}{c}\text { ProbandID, } \\
\text { TrialNo. }\end{array}$} & \multirow{2}{*}{ Date, Time } & \multirow{2}{*}{$\begin{array}{l}\text { TestExec [SimSick], } \\
\text { TestExec [VRT est] }\end{array}$} & \multirow{2}{*}{ TestID, DataFileName } & \multirow{2}{*}{ T_Score } & $\Delta$ \\
\hline 32 & & & & & & \\
\hline 33 & 42,1 & $15.03 .99,13: 50: 45$ & No, Yes & P5, 42_1.nav & & \\
\hline 34 & 42,2 & $15.03 .99,14: 12: 50$ & No, Yes & P1, 42_2nav & & \\
\hline 35 & 42,3 & $15.03 .99,14: 16: 43$ & No, Yes & P1, 42_3.nav & & \\
\hline 36 & 42,4 & $15.03 .99,14: 19: 05$ & No, Yes & P1, 42_4.nav & & \\
\hline 30 & 42,5 & $15.03 .99,14: 21: 28$ & No, Yes & P1, 42_5nav & & \\
\hline 37 & 42,6 & $15.03 .99,14: 23: 46$ & No, Yes & P1, 42_6.nav & & \\
\hline 38 & 42,7 & $15.03 .99,14: 29: 47$ & No, Yes & H5, 42_7.nav & & \\
\hline 39 & 42.8 & $15.03 .99,14: 33: 22$ & No, Yes & H3, 42_8nav & & \\
\hline 40 & 42,9 & $15.03 .99,14: 35: 51$ & No, Yes & H3, 42_9.nav & & \\
\hline 41 & 42,10 & $15.03 .99,14: 46: 01$ & No, Yes & H3, 42_10.nav & & \\
\hline 42 & 42,11 & $15.03 .99,14: 48: 43$ & No, Yes & H3, 42_11.nav & & \\
\hline 42 & 42,12 & $15.03 .99,14: 51: 16$ & No, Yes & H3, 42_12.nav & & \\
\hline 43 & 42,13 & $15.01 .00,22: 38: 12$ & No, Yes & H1, 42-13.nav & & $\nabla$ \\
\hline
\end{tabular}


Abb.6.3. Generierung eines neuen Testdurchlaufs und Aufruf des VR-Tests mit korrespondierendem Simulator Sickness Questionnaire

Virtual Reality-Supported Neuropsychological Test Environment - Version 1.0

Print Exxit

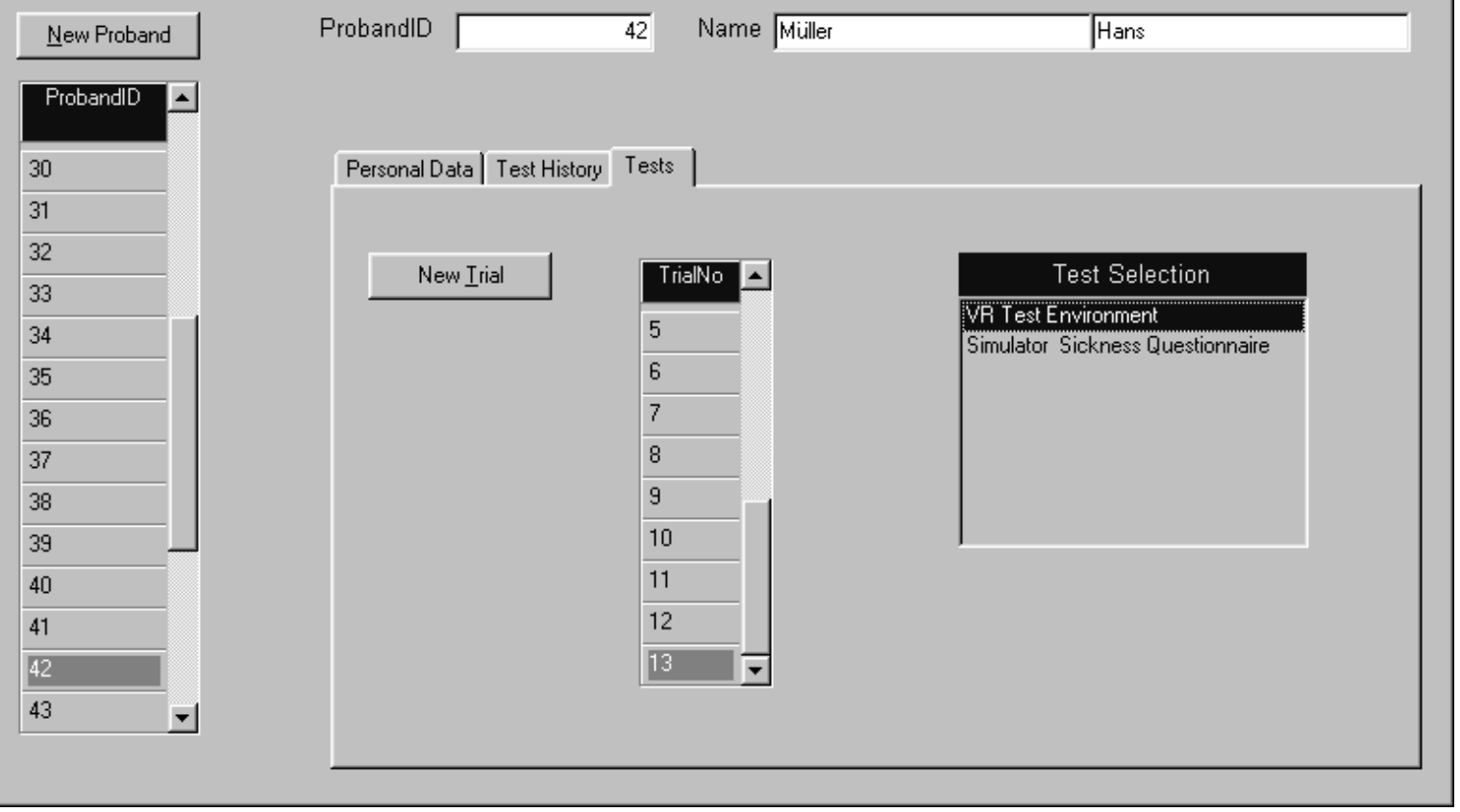


Abb. 6.4. Eingabe der SSQ Daten

\begin{tabular}{|c|c|c|c|c|c|}
\hline \multicolumn{6}{|c|}{ Simulator Sickness Questionnaire } \\
\hline \multicolumn{6}{|c|}{ PLEASE SCORE } \\
\hline \multicolumn{4}{|c|}{-General discomfort } & & Difficulty concentrating \\
\hline co & ○ 1 & $\subset 2$ & $\mathrm{C}_{3}$ & & ○ 3 \\
\hline \multicolumn{4}{|c|}{ Fatigue } & & Fullness of head \\
\hline co & $\odot 1$ & $\odot 2$ & c 3 & & c 3 \\
\hline \multicolumn{4}{|c|}{ Headache } & & Blurred vision \\
\hline co & $\odot 1$ & ${ }^{2}$ & ○ 3 & & $c_{3}$ \\
\hline \multicolumn{4}{|c|}{ Eyestrain } & & Dizzy (eyes open) \\
\hline co & ○ 1 & $\mathrm{C}_{2}$ & $c_{3}$ & & $c_{3}$ \\
\hline \multicolumn{4}{|c|}{-Difficulty focusing } & & Dizzy (eyes closed) \\
\hline co & ○ 1 & $\odot 2$ & c 3 & & $c_{3}$ \\
\hline \multicolumn{4}{|c|}{ Increased salivation } & & Vertigo \\
\hline co & $\odot 1$ & $\odot 2$ & ○ 3 & & $c_{3}$ \\
\hline \multicolumn{4}{|c|}{ Sweating } & & Stomache awareness \\
\hline co & $\odot 1$ & $\mathrm{C} 2$ & $c_{3}$ & & $\mathrm{c}_{3}$ \\
\hline \multicolumn{4}{|c|}{ Nausea } & & Burping \\
\hline co & ○ 1 & $\mathrm{C} 2$ & c 3 & & $\mathrm{C} 3$ \\
\hline & & & & & \\
\hline
\end{tabular}

Abb. 6.5. Auswahl der Testumgebung mit VR-Konfiguration

\section{C) VR Test Environment}

Choose version:

\begin{tabular}{|c|c|}
\hline Projektion Park A & $\nabla$ \\
\hline $\begin{array}{l}\text { HMD Park B } \\
\text { HMD Maze B } \\
\text { HMD Training Maze } \\
\text { HMD Training Maze }\end{array}$ & $\Delta$ \\
\hline Projektion Park $\mathrm{A}$ & \\
\hline $\begin{array}{l}\text { Projektion Maze A } \\
\text { Projektion Park B }\end{array}$ & 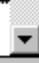 \\
\hline
\end{tabular}




\subsection{Beispiel eines Wegenetzes der Testumgebungen}

Abb. 6.6. Wegenetz Labyrinth $(S=$ Startpunkt, $Z=$ Zielpunkt $)$

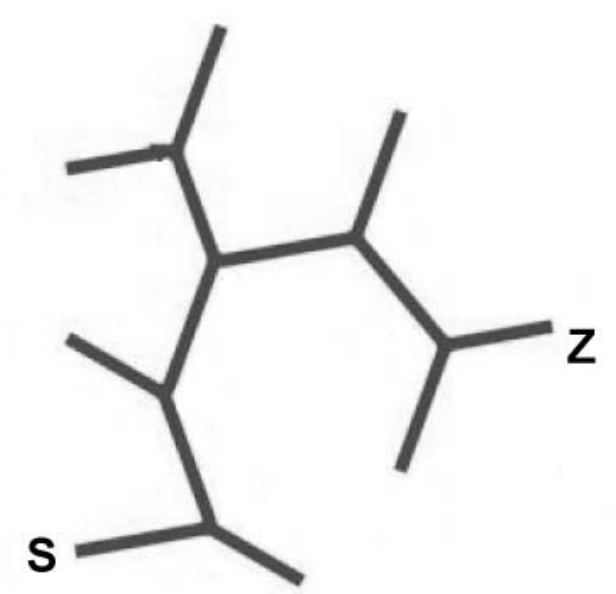

6.13. Bildwiederholungsrate (engl. frame rate) komplexer VRUmgebungen auf verschiedenen Computersystemen

\begin{tabular}{|c|c|c|c|c|}
\hline Rechnerbezeichnung & $\begin{array}{l}\text { Frame Rate Park } \\
\text { Mittelwert }\end{array}$ & $\begin{array}{l}\text { Frame Rate Maze } \\
\text { Mittelwert }\end{array}$ & $\begin{array}{l}\text { Preis Ausstattung } \\
\text { (ca. in tsd Euro) }\end{array}$ & $\begin{array}{l}\text { Markteintritt } \\
\text { des Rechners }\end{array}$ \\
\hline $\begin{array}{l}\text { Intergraph Workstation } \\
2 \text { x Pentium II } 300 \mathrm{MHz}, 256 \mathrm{MB} \text { RAM, } \\
2 \text { OpenGl-Grafikkarten mit } 64 \mathrm{MB} \\
\text { Speicher und } 32 \mathrm{MB} \text { Textur-Speicher, } \\
\text { Geometriebeschleuniger, } \\
\text { Windows NT } 4.0\end{array}$ & 13,0 & 14,6 & 30 & 1997 \\
\hline $\begin{array}{l}\text { Standard PC } \\
2 \text { x Pentium II } 350 \mathrm{MHz}, 128 \mathrm{MB} \text { RAM, } \\
\text { Diamond FireGI } 4000 \text { Grafikkarte, } \\
\text { Windows NT } 4.0\end{array}$ & 9,24 & 12,8 & 2 & 1999 \\
\hline Dto., mit Vodoo2 3D-AddOn-Grafikkarten * & 25,0 & 36,1 & 2,2 & 1999 \\
\hline $\begin{array}{l}\text { Standard PC } \\
\text { AMD K6.2 } 300 \mathrm{MHz}, 64 \mathrm{MB} \text { RAM, } \\
\text { ELSA Erazor III (Riva TNT2) } \\
\text { Grafikkarte } \\
\text { Windows } 98\end{array}$ & 8,4 & 22,0 & 1,5 & 1999 \\
\hline $\begin{array}{l}\text { SGI Visual Workstation 320, } \\
2 \text { x Pentium III } 450 \mathrm{MHz}, 512 \mathrm{MB} \text { RAM, } \\
\text { Cobalt Chipsatz, Windows NT } 4.0\end{array}$ & 8,6 & 11,6 & 6 & 1999 \\
\hline
\end{tabular}

* Rechner, der in der präklinischen und klinischen Evaluation eingesetzt wurde 


\section{Literaturverzeichnis}

Aguirre GK, D’Esposito M (1997): Environmental Knowledge Is Subserved by Separable Dorsal/Ventral Neural Areas. J Neurosci 17(7), 2512-2518

Aguirre GK, D’Esposito M (1999): Topographical disorientation: a synthesis and taxonomy. Brain 122, 1613-1628

Aguirre GK, Zahran E, D'Esposito M (1998): Neural components of topographical representation. Proc Natl Acad Sci USA 95, 839.846

Akatsuka Y, Shibasaki T, Saito A, Kosaka A, Masuzaki H, Asano T, Furuhashi Y: Navigation System for Neurosurgery with PC Platform; in: Medicine Meets Virtual Reality 2000 (Studies in Health Technology and Informatics, Vol. 70); hrsg v. Westwood JD, Miller Hoffman H, Mogel GT, Robb RA, Stredney D; IOS Press, Amsterdam 2000, 10.16

Anonymous (1991): Being and believing: ethics of virtual reality. Lancet $\underline{338}$, 283.284

Astur RS, Ortiz ML, Sutherland RJ (1998): A characterization of performance by men and women in a virtual Morris water task: A large and reliable sex difference. Behav Brain Res $\underline{93}$, $185 \cdot 190$

Astur RS, Taylor LB, Mamelak AN, Philpott L, Sutherland RJ (2002): Humans with hippocampus damage display severe spatial memory impairments in a virtual Morris water test. Behav Brain Res 132, 77.84.

Barfield W, Hendrix C, Bjorneseth O, Kaczmarek K, Lotens W (1995): Comparison of human sensory capabilities with technical specifications of virtual environment equipment. Presence Teleoper Virtual Environ 4 , 329-356

Bäumler G: Lern- und Gedächtnistest (LGT-3). 1. Auflage. Hogrefe Verlag, Göttingen 1974, ohne Seitenangabe

Beaumont JG: The Aims of Neuropsychological Assessment; in: Assessment in Neuropsychology; hrsg. v. Harding L, Beech JR; Routledge, London-New York 1996, 3-15

Berlage T: Augmented reality for diagnosis based on ultrasound images; in: CVRMed-MRCAS '97 - First International Joint Conference on Computer Vision, Virtual reality and Robotics in Medicine and Medical Robotics and Computer Assisted Surgery; hrsg. v. Troccaz J, Grimson E, Mösges R; Springer, Berlin 1997, 253-262

Biocca F (1992): Will simulation sickness slow down the diffusion of virtual environment technology? Presence Teleoper Virtual Environ 1, 334-343 
Birrell ND, Ould MA: A practical handbook for software development. Cambridge University Press, Cambridge 1986

Blanquer I, Hernandez V, Ramirez FJ, Vidal A: Parallel Segmentation and Rendering Using Clusters of PCs; in: Medicine Meets Virtual Reality 2000 (Studies in Health Technology and Informatics, Vol. 70); hrsg v. Westwood JD, Miller Hoffman H, Mogel GT, Robb RA, Stredney D; IOS Press, Amsterdam 2000, 33.35

Bles W, Bos JE, Kruit H (2000): Motion sickness. Curr Opin Neurol 13, 19.25

Botella C, Banos RM, Perpina C, Villa H, Alcaniz M, Rey A (1998): Virtual reality treatment of claustrophobia: a case report. Behav Res Ther $\underline{36}$, 239-246

Bryson S: Approaches to the successful design and implementation of VR applications; in: Virtual Reality Applications; hrsg. v. Earnshaw RA, Vince JA, Jones H; Academic Press, London 1995, 9.1-9.11

Buckwalter JG, Rizzo A: Virtual reality and the neuropsychological assessment of persons with neurologically based cognitive impairments; in: Studies in Health Technology and Informatics (Vol. 39); hrsg. v. Morgan KS, Hoffman HM, Stredney D, Weghorst SJ; IOS Press, AmsterdamOxford-Tokyo-Washington DC 1997, 17.33

Bullinger HJ, Bauer W: Strategische Dimensionen der Virtual Reality; in: Proceedings IPA/ IAO. Forum: Virtual Reality '94 - Anwendungen \& Trends; o. Hrsg.; Springer, Stuttgart 1994, ohne Seitenangaben

Burgess N (2002): The hippocampus, space, and viewpoints in episodic memory. Q J Exp Psychol A $\underline{55}, 1057-1080$.

Carrozzo M, Lacquaniti F (1998): Virtual reality: a tutorial. Electroencephalogr Clin Neurophysiol $\underline{109}, 1.9$

Christiansen C, Abreu B, Ottenbacher K, Huffman K, Brent M, Culpepper R (1998): Task Performance in Virtual Environments Used for Cognitive Rehabilitation After Traumatic Brain Injury. Arch Phys Med Rehabil 79, 888.892

Chung SM (2002): Safety Issues in Magnetic Resonance Imaging. J Neurol Ophtalmo 22, 35 . 39.

Clemens SA, Howarth PA: Changes in virtual simulator sickness susceptibility over the menstrual cycle. Vortrag; 36 ${ }^{\text {th }}$ United Kingdom Group Meeting on Human Responses to Vibration; Farnborough/UK, 12.14. September 2001, Onlinedokument: http://www.lboro.ac.uk/departments/hu/groups/viserg/viserg1.htm

Cobb SVG, Nichols S, Ramsey A, Wilson JR (1999). Virtual Reality-Induced Symptoms and Effects (VRISE). Presence Teleoper Virtual Environ 8(2), 169-186 
Cox DJ, Quillian WC, Thorndike FP, Kovatchev P, Hanna G (1998): Evaluating Driving Performance of Outpatients with Alzheimer Disease. J Am Board Fam Pract 11(4), 264-271

Cruz-Neira C, Sandin DJ, DeFanti TA: Surround-screen projection-based virtual reality: The design and implementation of the CAVE; in: Proceedings ACM SIGGRAPH '93; O. Hrsg.; ACM, Anaheim CA 1993, ohne Seitenangaben

Deisinger J, Cruz-Neira C, Riedel O, Symanzik J: The effect of different viewing devices for the sense of presence and immersion in virtual environments: a comparison of stereoprojections based on monitors, HMDs and screens; in: Design of Computing Systems: Social and Ergonomic Considerations (Advances in Human Factors/Ergonomics, Vol. 21B); hrsg. v. Smith MJ, Salvendy G, Koubek RJ; Elsevier Science, Amsterdam 1997, 881.884

Denenberg VH, Talgo N, Carroll DA, Freter S, Deni R (1991): A computer-aided procedure for

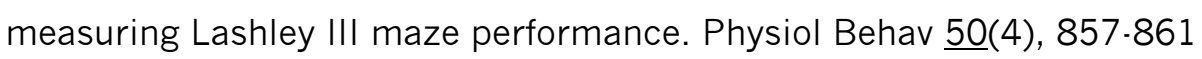

DiZio P, Lackner JR (1992): Spatial Orientation, Adaption and Motion Sickness in Real and Virtual Environments. Presence Teleoper Virtual Environ $\underline{3}(1), 319 \cdot 328$

Dobie T, McBride D, Dobie T jr, May J (2001): The effects of age and sex on susceptibility to motion sickness. Aviat Space Environ Med 72, 13.20

Durlach NI, Mavor AS. Virtual Reality. Scientific and Technological Changes. National Academy Press, Washington D.C. 1995, S. $395 f$

Fenlon HM, Nunes DP, Schroy PC III, Barish MA, Clarke PD, Ferrucci JT (1999): A Comparison of Virtual and Conventional Colonoscopy for the Detection of Colorectal Polyps. N Engl J Med $\underline{341}(20), 1496-1503$

Ferrucci JT (2001): Colon cancer screening with virtual colonoscopy: promise, polyps, politics. Am J Roentgenol $2001 \underline{177(5), 975.988}$

FHG-IAO: Personal Immersion. Projektbeschreibung; Fraunhofer Institut für Arbeitswirtschaft und Organisation, Stuttgart 2001

Fox S, Arnon I (1988): Motion sickness and anxiety. Aviat Space Environ Med 59(8), 728-33

Gahlinger PM (1999): Motion Sickness - How to help your patients to avoid travel travail. Postgrad Med 106(4), 177.184

Garcia-Palacios A, Hoffman H, Carlin A, Furness III TA, Botella C (2002): Virtual reality in the treatment of spider phobia: a controlled study. Behav Res Ther $\underline{40}$, 983.993

Gillner S, Mallot HA (1998): Navigation and Acquisition of Spatial Knowledge in a Virtual Maze. J Cog Neurosci 10(4), 445.463 
Glantz K, Durlach NI, Barnett RC, Aviles WA (1997): Virtual reality (VR) and psychotherapy: opportunities and challenges. Presence Teleoper Virtual Environ $\underline{6}$, 87-105

Gomoll K: Some Techniques for Observing Users; in: The Art of Human-Computer Interace Design; hrsg. v. Laurel B; Addison-Wesley Reading MA 1990, 85-90

Grimsdale C: Virtual Reality - key technologies, problems and emerging solutions; in: Virtual Reality International 1992. Impacts and Application; hrsg. v. Feldman T; Meckler, London 1992, 14-22

Grön G, Wunderlich AP, Spitzer M, Tomczak R, Riepe MW (2000): Brain activation during human navigation: gender-different neural networks as substrate of performance. Nat Neurosci $\underline{3}(4), 404.408$

Grönemeyer DHW, Seibel RMM, Schmidt A, Melzer A, Deli M: Two and three dimensional imaging for interventional MRI and CT guidance; in: Studies in Health Technology and Informatics, Vol. 29; hrsg. v. Weghorst SJ, Sieburg HB, Morgan SB ; IOS Press, AmsterdamOxford-Tokyo-Washington DC 1996, 62.76

Grossmann M, Skinner MH (1996): A simple computer based system to analyze Morris water maze trials on-line. J Neurosci Methods $\underline{70(2), 171-175}$

Haberland N, Kalff R, Ebmeier K (1997): Neuronavigation in der Tumorchirurgie des zentralen Nervensystems. Onkologe $\underline{3}(2), 143.147$

Hartley T, Maguire EA, Spiers HJ, Burgess N (2003): The well-worn route and the path less travelled: distinct neural bases of route following and wayfinding in humans. Neuron $\underline{37}, 877$. 888.

Hendrix C, Barfield W (1996): Presence within Virtual Environments as a Function of Visual Display. Presence Teleoper Virtual Environ 5(3), 274-289

Hettinger LJ, Riccio GE (1992): Visually Induced Motion Sickness in Virtual Environments. Presence Teleoper Virtual Environ 1(3), 306-310

Holdstock JS, Mayes AR, Cezayirli E, Isaac CL, Aggleton JP, Roberts N (2000): A comparison of egocentric and allocentric spatial memory in a patient with selective hippocampal damage. Neuropsychologia $\underline{38}$, 410-425.

Hoplight BJ 2nd, Boehm GW, Hyde LA, Deni R, Denenberg VH (1996): A computer-aided procedure for measuring Hebb-Williams maze performance. Physiol Behav $\underline{60}(4), 1171-1176$

Insko BE: Measuring Presence: Subjective, Behavioral and Physiological Methods; in: Being There: Concepts, effects and measurement of user presence in synthetic environments; hrsg. v. Riva G, Davide F, Jsselsteijn WJ; los Press, Amsterdam NL 2003, 109.119 
Jaeger BK, Mourant RR: Comparison of Simulator Sickness Using Static and Dynamic Walking Simulators; in: Proceedings of the Human Factors and Ergonomics Society, 45th Annual Meeting; Human Factors and Ergonomics Society, Santa Monica, CA 2001, 1896-1900

John NW, Phillips N:Surgical Simulators Using the WWW; in: Medicine Meets Virtual Reality 2000. Studies in Health Technology and Informatics, Vol. 70; hrsg v. Westwood JD, Miller Hoffman H, Mogel GT, Robb RA, Stredney D; IOS Press, Amsterdam 2000, 146-152

Johnson DA, Rose FD, Rushton S, Pentland B, Attree EA (1998): Virtual Reality: A new prosthesis for brain injury rehabilitation. Scott Med J $\underline{43}, 81.83$

Jokerst MD, Gatto M, Fazio R, Gianaros PJ, Stern RM, Koch KL (1999): Effects of gender of subjects and experimenter on susceptibility to motion sickness. Aviat Space Environ Med $\underline{70}$, 962.965

Kahana MJ, Sekuler R, Caplan JB, Kirschen M, Madsen JT (1999): Human theta oscillations exhibit task dependence during virtual maze navigation. Nature 399, 781.784

Kaltenborn KF, Rienhoff O (1993): Virtual Reality in Medicine. Methods Inf Med 32, 407-17

Kennedy RS, Lane NE (1993): Simulator sickness questionnaire: an enhanced method for quantifying simulator sickness. Int J Aviat Psychol $\underline{3}(3), 203-220$

Kennedy RS, Lane NE, Lilienthal MG, Berbaum KS, Hettinger LJ (1992): Profile Analysis of Simulator Sickness Symptoms: Application to Virtual Environment Systems. Presence Teleoper Virtual Environ 1(3), 295-301

Kerkhoff F: Räumlich-perzeptive, räumlich-kognitive, räumlich-konstruktive und räumlichtopographische Störungen; in: Lehrbuch der Klinischen Neuropsychologie; hrsg. v. Sturm W, Hermann M, Wallesch CW. Swets \& Zeitlinger, Lisse/NL 2000, 411.429

Kindsmüller M, Kaindl A, Schuri U, Zimmer A (1999): Topographische Orientierung bei Patienten mit erworbener Hirnschädigung. Z Neuropsychol 10(2), 77.86

King JA, Burgess N, Hartley T, Vargha-Khadem F, O'Keefe J (2002): Human hippocampus and viewpoint dependence in spatial memory. Hippocampus 12, 811-820.

Kolasinski EM: Simulator Sickness in Virtual Environment (Technical Report 1027); US Army Research Institute for Behavioural and Social Sciences, Alexandria/Virginia - USA 1995

Kolasinski EM: Prediction of Simulator Sickness in a Virtual Environment. PhD Dissertation, University of Central Florida, Orlando/Florida 1996

Kolasinski EM, Gilson RD (1999): Ataxia Following Exposure to a Virtual Environment. Aviat Space Environ Med 70(3), 264-269 
Kuhlen T, Kraiss KF, Szymanski A, Dohle C, Hefter H, Freund HJ: Virtual Holography in Diagnosis and Therapy of Sensorimotor Disturbances; in: Studies in Health Technology and Informatics, Vol. 29; hrsg. v. Weghorst SJ, Sieburg HB, Morgan SB; IOS Press, Amsterdam. Oxford-Tokyo-Washington DC 1996, 184-193

Kushniruk AW, Patel VL, Cimino JJ: Usability Testing in Medical Informatics: Cognitive Approaches to Evaluation of Information Systems and User Interfaces ; in: Proceedings of the 1997 AMIA Annual Fall Symposium; hrsg. v. Masys DR; Hanley \& Belfus, Philadelphia 1997, 218.222

Leimann Patt HO, Baistrochi RL, Moia PL (1988): Neuropsychiatric observations of proprioceptive sensitivity in motion sickness susceptibility. Aviat Space Environ Med $\underline{59}(11)$, 1083.88

Lessiter J, Freeman J, Keogh E, Davidoff J (2001): A Cross-Media Presence Questionnaire: The ITC-Sense of Presence Inventory. Presence Teleoper Virtual Environ 10(3), 282.297

Lewis $\mathrm{CH}$, Griffin MJ: Human Factors Consideration in Clinical Applications of Virtual Reality; in: Studies in Health Technology and Informatics, Vol. 29; hrsg. v. Morgan KS, Hoffman HM, Stredney D, Weghorst SJ; IOS Press, Amsterdam-Oxford-Tokyo-Washington DC 1997, 35-56

Lezak MD. Neuropsychological Assessment (3rd Ed.). Oxford University Press, New York 1995

Ludwig A, Verhey JF: Transfer of sonographical Data to a Head Mounted Display (HMD) Advantages for Head and Neck Surgery; in: Proceedings of the 14th International Congress and Exhibition, Computer Assisted Radiology and Surgery (CARS 2000), San Francisco, CA, USA; hrsg. v. Lemke LU, Vannier MW, Inamura K, Farman AG, Doi K; Elsevier Science, Amsterdam $2000,60-65$

Maguire EA, Burke T, Phillips J, Staunton H (1996). Topographical disorientation following unilateral temporal lobe lesions in humans. Neuropsychologia 34(10), 993-1001

Maguire EA, Frith CD, Burgess N, Donnett JG, O'Keefe JO (1998): Knowing Where Things Are: Parahippocampal Involvement in Encoding Object Locations in Virtual Large-Scale Space. J Cog Neurosci 10(1), 61.76

Mattes-Cramon G, Cramon Y von: Störungen exekutiver Funktionen; in: Lehrbuch der Klinischen Neuropsychologie; hrsg. v. Sturm W, Hermann M, Wallesch CW; Swets \& Zeitlinger, Lisse (NL) 2000, 392-411

McKenna M, Zeltzer D (1992): Three Dimensional Visual Display Systems for Virtual Environments. Presence Teleoper Virtual Environ 1(4), 421.458

Mehlitz M, Weniger G, Kleinoeder T, Rienhoff O (1998): Virtual Reality in der medizinischen Anwendung - Perspektiven für die Krankenversorgung. Krankenhaus $\underline{8}$, 463.468 
Morgan KS, Hoffman HM, Stredney D, Weghorst SJ: Global Healthcare Grid (Studies in Health Technology and Informatics, Vol. 39); IOS Press, Amsterdam-Oxford-Tokyo-Washington DC 1997

Morrison PR, Gates GR (1988): Assessment of a microcomputer-based version of the Austin maze. J Gen Psychol 115(3), 307-314

Müller WK, Ziegler R, Bauer A, Soldner EH (1995): Virtual Reality in Surgical Arthroscopic Training. J Image Guid Surg $\underline{1}$, 288-294

Münte S, Hollenhorst J, Friedrich L, Heine J, Leuwer M, Piepenbrock S, Becker H (2002): Intranasale Midazolamapplikation zur Anxiolyse bei der Magnetresonanztomographie. Klin Neurorad $\underline{12}, 82.87$

Naepflin U, Menozzi M (1998): VFX1-Datenhelm im Vergleich mit 17" Monitor. Effekte der

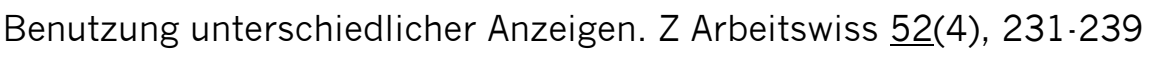

Nichols S, Patel H (2002): Health and safety implications of virtual reality: a review of empirical evidence. Appl Ergon 33, 251-271

OpenGL Performance Characterization Group: Results of the OPC-AWadvs-01 test. Report; The OpenGL Performance Characterization Group 1998, ohne Seitenangabe

Ordrige RJ, Fullerton G, Norris DG (2000): MRI safety limits: is MRI safe or not? Br J Radiology $\underline{73}, 1-2$

Pagel B.U, Six H-W: Software Engineering. Die Phasen der Softwareentwicklung. AddisonWesley, Bonn-Paris-Reading/Massachusetts 1994

Park AH, Hu S (1999): Gender differences in motion sickness history and susceptibility to optokinetic rotation-induced motion sickness. Aviat Space Environ Med 70, 1077-1080

Pausch R, Crea T, Conway M (1992): A literature survey for virtual environments: military flight simulator visual systems and simulator sickness. Presence Teleoper Virtual Environ $\underline{1}$, 344-363

Pimentel K, Teixeira K: Virtual Reality - Through the new looking glasses. McGraw Hill, New York-San Francisco 1995

Pimentel de Sampaio Goes V, Santos Machado L dos, Calixto Cabral M, Bittencourt Vidigal Leitao R, Deus Lopes R de, Knörich Zuffo M: Interactive Stereoscopic Full-Color Direct Volume Visualization for Virtual Reality Applications in Medicine; in: Medicine Meets Virtual Reality 2001 (Studies in Health Technology and Informatics, Vol. 81); hrsg. v. Westwood JD, Miller Hoffman H, Mogel GT, Stredney D, Robb RA; IOS Press, Amsterdam 2001, 161-167

Porteus SD: The maze test and clinical psychology. Pacific Books, Palo Alto CA 1959 
Poucet B (1993): Spatial Cognitive Maps in Animals: New Hypotheses on Their Structure and Neural Mechanisms. Psychol Rev $\underline{100}(2), 163.182$

Prothero JD, Draper MH, Furness TA, Parker DE, Wells MJ (1999): The Use of an Independent Visual Background to Reduce Simulator Side Effects. Aviat Space Environ Med 70(3), 277.283

Pugnetti L, Mendozzi L, Barbieri E, Rose FD, Attree EA (1995a). Nervous system correlates of virtual reality experience; in: Proc 1st Euro Conf Disab Virt Reality \& Assoc Tech; hrsg. v. Sharkey PM; Maidenhead UK, 239-246

Pugnetti L, Mendozzi L, Motta A, Cattaneo A, Barbieri E, Brancotti A (1995b): Evaluation and retraining of adults cognitive impairments: which role for virtual reality technology? Comput Biol Med 25(2), 213.227

Pugnetti L, Mendozzi L, Barbieri E, Motta A: VR Experience with neurological patients: basic cost/benefits issues; in: Studies in Health Technology and Informatics, Vol. 29; hrsg. v. Riva G, Wiederhold BK, Molinari E ; IOS Press, Amsterdam-Oxford-Tokyo-Washington DC 1998, 243. 248

Regan EC, Price KR (1994): The frequency of occurrence and severity of side-effects of immersion virtual reality. Aviat Space Environ Med 65, 527-30

Regan EC: Some human factors issues in immersive virtual reality: facts and speculation; in: Virtual Reality Applications; hrsg. v. Earnshaw RA, Vince JA, Jones H; Academic Press 1995, 163.184

Riecke BE, van Veen HAHC, Bülthoff HH (2002): Visual Homing Is Possible Without Landmarks: A Path Integration Study In Virtual Reality. Presence 11(5), 443.473

Riedel O: Immersive Projection Systems for Virtual Environments - Benefits for the Industry. Fraunhofer Institut für Arbeitswirtschaft und Organisation, Stuttgart 1997

Ring H (1998): Is neurological rehabilitation read for immersion in the world of virtual reality? Disab Rehab 20(3), 98-101

Riva G (1997): The Virtual Environment for Body-Image Modification (VEBIM): Development and Preliminary Evaluation. Presence Teleoper Virtual Environ $\underline{6}(1), 106.117$

Riva G (1998): Modifications of Body-Image Induced by Virtual Reality. Percept Mot Skills $\underline{86}$, 163.170

Rizzo AA, Wiederhold M, Buckwalter JG: Basic issues in the use of virtual environments for mental health applications; in: Virtual Environments in Clinical Psychology and Neuroscience; hrsg. v. Riva G, Wiederhold BK, Molinari E; IOS Press Amsterdam 1998a, 21.42 
Rizzo AA, Buckwalter JG, Neumann U, Kesselman C, Thiebaux M (1998b): Basic issues in the application of Virtual Reality for the assessment and rehabilitation of cognitive impairments and functional disabilities. CyberPsychol Behav $\underline{1}(1), 59.78$

Rizzo AA, Bowerly T, Buckwalter JG, Schultheis M, Matheis R: Virtual Environments for the Assessment of Attention and Memory: The Virtual Classroom and Office; in: Proceedings of the International Conference on Disability, Virtual Reality and Associated Technology; hrsg. v. Sharkey PM; Vesaprem, Hungary, 2002, ohne Seitenangaben

Robb RA: Virtual (computed) endoscopy: development and evaluation using the Visible Human Datasets; in: The Visible Human Project Conference Proceedings; hrsg. v. Banvard RA; National Library of Medicine (US), Office of High Performance Computing and Communications Bethesda (MD) 1996, ohne Seitenangaben

Rose FD, Johnson DA, Attree EA (1997): Rehabilitation of the head-injured child: basic research and new technology. Pediat Rehabil $1(1), 3 \cdot 7$

Rothbaum BO, Hodges L, Kooper R, Opdyke D, Williford M, North M (1995): Effectiveness of computer-generated (virtual reality) graded exposure in the treatment of acrophobia. Am J Psychiatry 152, 626.628

Ruddle RA, Payne SJ, Jones DM (1998): Navigating Large-Scale 'Desktop' Virtual Buildings: Effects of Orientation Aids and Familiarity. Presence Teleoper Virtual Environ $\underline{7}(2), 179.192$

Sandstrom NJ, Kaufman J, Huettel SA (1998): Males and females use different distal cues in a virtual environment navigation task. Cog Brain Res $\underline{6}, 351-360$

Saranummi N: Supporting System Development with Technology Assessment; in: Assessment and Evaluation of Information Technology in Medicine; hrsg. v. Van Gennip EMSJ, Talmon JL; IOS Press, Amsterdam-Oxford-Tokyo-Washington DC 1995, 35.49

Sheridan TB (1992). Musings on Telepresence and Virtual Presence. Presence Teleoper Virtual Environ 1(1), 120-126

Shin H, Stamm G, Högemann D, Galanski M (2000): Grundregeln der Datenakquisition und Datennachverarbeitung für die Erstellung hochwertiger virtueller Modelle. Radiologe 40, 304 . 312

Skelton RW, Bukach CM, Laurance HE, Thomas KGF, Jacobs WJ (2000): Humans with traumatic brain injuries show place-learning deficits in computer-generated virtual space. J Clin Exp Neuropsychol 22(2), 157-175

Slater M, Usoh M, Steed A (1994): Depth of Presence in Virtual Environments. Presence Teleoper Virtual Environ $\underline{3}(2), 130-144$ 
Smith EE, Jonides J (1999): Storage and executive processes in the frontal lobes. Science 283, $1657 \cdot 1660$

So RHY, Lo WT, Ho ATK (2001): Effects of Navigation Speed on Motion Sickness Caused by an Immersive Virtual Environment. Hum Factors 43(3), 452-461

Spiers HJ, Burgess N, Maguire EA, Baxendale SA, Hartley T, Thompson PJ, O'Keefe JO (2001): Unilateral temporal lobectomy show lateralized topographical and episodic memory deficits in a virtual town. Brain 124, 2476-2489

SPSS für Windows, Release 10.0.7 [Software]. SPSS Inc., Chicago/IIlinois 2001

Stanney KM, Hash P (1998): Locus of User-Initiated Control in Virtual Environments: Influences on Cybersickness. Presence Teleoper Virtual Environ $\underline{7}(5)$, 447.459

Stanney K, Salvendy G, Deisinger J, DiZio P, Ellis S, Ellison J, Fogleman G, Gallimore J, Singer M, Hettinger L, Kennedy R, Lackner J, Lawson B, Mead A, Mon-Williams M, Newman D, Piantanida T, Reeves L, Riedel O, Stoffregen T, Wann J, Welch R, Wilson J, Witmer B (1998): Aftereffects and sense of presence in virtual environments: formulation of a research and development agenda. Int J Hum Comput Interact 10(2), 135.187

Stanton D, Foreman N, Wilson PN: Uses of virtual reality in clinical training: developing the spatial skills of children with mobility impairments; in: Studies in Health Technology and Informatics, Vol. 29; hrsg. v. Riva G, Wiederhold BK, Molinari E; IOS Press, Amsterdam. Oxford-Tokyo-Washington DC 1998, 219.32

Stuss DT, Alexander MP (2000): Executive functions and the frontal lobes: a conceptual view. Psychological Research $\underline{63}, 289.298$

Stuss DT, Benson DF: The frontal lobes. Raven, New York 1986

Sutherland IE: A head-mounted three-dimensional display; in: Fall Joint Computer Conference, AFIPS Conference Proceedings Vol. 33(1); o. Hrsg.; Thompson Book, Washington (DC) 1968, 757.764

Székely G, Brechbühler C, Hutter R, Rhomberg A, Schmid P: Modelling of Soft Tissue Deformation for Laparoscopic Surgery Simulation; in: Proceedings of the MICCAI '98 Conference - Medical Image Computing and Computer-Assisted Intervention; hrsg. v. Wells WM, Colchester ACF, Delp S; Springer, Heidelberg 1998, 550-561

Székely G, Satava RM (1999): Virtual Reality in medicine. BMJ $\underline{319}$, 1305-1308

Tasto JL, Verstreken K, Brown M, Bauer JJ (2000). PreOP Endoscopy Simulator: From Bronchoscopy to Ureteroscopy; in: Medicine Meets Virtual Reality 2000 (Studies in Health Technology and Informatics, Vol. 70); hrsg v. Westwood JD, Miller Hoffman H, Mogel GT, Robb RA, Stredney D; IOS Press, Amsterdam 2000, 344-349 
Tranel D: Development of the concept of 'executive function' and its relationship to frontal lobes; in: Handbook of Neuropsychology (Vol. 9); hrsg. v. Boller, Grafman: Elsevier, Amsterdam 1994, 125-141

Ungerleider LG, Courtney SM, Haxby JV (1998): A neural system for human visual working memory. Proc Natl Acad Sci USA 95, 883.890

Veen HAHC van, Distler HK, Braun SJ, Bülthoff HH: Navigating through a virtual city: using virtual reality technology to study human action and perception. Technical Report No. 57, MaxPlanck-Institut für biologische Kybernetik, Tübingen 1998

Verhey JF, Ludwig A, Rexilius J, Warfield SK, Mamisch CT, Kikinis R, Westin CF, Seibel R, Rienhoff O (2002). Multimodale nicht-rigide Registrierung von Ultraschall und MR-Bilddaten unter Verwendung eines biomechanischen Modells; in: Bildverarbeitung für die Medizin 2002, Algorithmen - Systeme - Anwendungen; hrsg v. Meiler M, Saupe D, Kruggel F, Handels H, Lehmann T: Springer, Heidelberg 2002, 310-313

VirtualResearch Inc.: V8 User Guide; o. Hrsg.; VirtualResearch Inc., Aptos/California 1998, ohne Seitenangabe

Waller D, Hunt E, Knapp D (1998): The Transfer of Spatial Knowledge in Virtual Environment Training. Presence Teleoper Virtual Environ $\underline{7}(2), 129.143$

Wann J, Mon-Williams M (1996): What does virtual reality NEED?: human factor issues in the design of three-dimensional computer environments. Int J Hum-Comp Stud 444, 829-847

Wartenberg F, May M, Péruch O: Spatial Orientation in Virtual Environments: Background Considerations and Experiments; in: Spatial Cognition - An interdisciplinary approach to representing and processing spatial knowledge; hrsg. v. Freksa C, Habel C, Wender K; Springer, Heidelberg 1998, 469.489

Weghorst SJ, Sieburg HB, Morgan SB (Hrsg.). Healthcare in the Information Age. Studies in Health Technology and Informatics, Vol. 29; IOS Press, Amsterdam-Oxford-Tokyo-Washington DC 1996

Welch RB, Blackmon TT, Liu A, Mellers BA, Stark LW (1996): The Effects of Pictorial Realism, Delay of Visual Feedback and Observer Interactivity on the Subjective Sense of Presence. Presence Teleoper Virtual Environ $\underline{5}(3), 263.273$

Westwood JD, Miller Hoffman H, Mogel GT, Robb RA, Stredney D (Hrsg.). Medicine Meets Virtual Reality 2000. Studies in Health Technology and Informatics, Vol. 70; IOS Press, Amsterdam 2000 
Westwood JD, Miller Hoffman H, Mogel GT, Stredney D, Robb RA (Hrsg.). Medicine Meets Virtual Reality 2001. Studies in Health Technology and Informatics, Vol. 81; IOS Press, Amsterdam 2001

Whalley LJ (1995): Ethical issues in the application of virtual reality to medicine. Comput Biol Med 25(2), 107-114

Witmer BG, Bailey JH, Knerr BW (1996): Virtual spaces and real world places: transfer of route knowledge. Int J Hum-Comp Stud $\underline{45}$, 413.428

Witmer BG, Klein PB (1998): Judging Perceived and Traversed Distance in Virtual Environments. Presence Teleoper Virtual Environ Z(2), 144-167

Witmer BG, Singer MJ (1998): Measuring Presence in Virtual Environments: A Presence Questionnaire. Presence Teleoper Virtual Environ Z(3), 225-240

Youngblut C, Johnson RE, Nash SH, Wienclaw RA, Will CA: Review of Virtual Environment Interface Technology. IDA Paper P.3186, Institute For Defense Analyses, Alexandria/Virginia, USA 1996

Zelkovitz MV: Stages in Software Development; in: Principles of Software Engineering and Design, hrsg. v. Zelkowitz MV, Shaw AC, Gannon JD; Prentice-Hall, Englewood Cliffs/New Jersey $1979,2 \cdot 11$ 


\section{Danksagung}

Herrn Prof. Dr. med. O. Rienhoff danke ich für die Überlassung des Dissertationsthemas und die Betreuung der vorliegenden Arbeit, Frau Prof. Dr. rer. nat. E. Irle (Abteilung Psychiatrie/ Schwerpunkt Neuropsychologie) für die Unterstützung der Durchführung dieser abteilungsübergreifenden Arbeit.

Für die kritische Mitbetreuung, die fachlich und persönlich gute Zusammenarbeit möchte ich mich vor allem bei Dr. med. Dipl. Psych. G. Weniger (Abt. Psychiatrie) bedanken, sowie bei Frau Dipl. Psych. S. Wolf (Abt. Psychiatrie), Britta v.d. Brincken, Dr. med. T. Kleinoeder (Medizinisches Rechenzentrum), Prof. (FH) Dr. med. T. Kesztyues (Fachhochschule UIm), Frau Dipl. Psych. U. Piccolo (Abt. Medizinische Informatik) und Dr. rer nat. J. Verhey (Abt. Medizinische Informatik).

Die in dieser Arbeit vorgestellten klinischen Ergebnisse wurden im Rahmen eines durch die Deutsche Forschungsgemeinschaft geförderten Projektes gewonnen (Rienhoff O, Irle E, Weniger G: VR-gestütztes neuropsychologisches Testsystem zur Diagnose und Therapie neuropsychiatrischer Erkrankungen. Geschäftszeichen RI 1000/1-1, Göttingen 1998). 


\section{Lebenslauf}

Am 22. Oktober 1972 wurde ich in Lüdinghausen/Westfalen als viertes Kind von Herrn Hubert Mehlitz und Frau Agnes Mehlitz, geb. Schlüter, geboren. Von 1979 bis 1983 besuchte ich die St. Ludgeri-Grundschule in Selm. 1992 legte ich das Abitur am Gymnasium Canisianum in Lüdinghausen/Westfalen ab.

Meinen Zivildienst leistete ich 1992/93 beim DRK Rettungsdienst Lünen mit Abschluss zum Rettungssanitäter. Nach Beginn eines Maschinenbaustudiums an der RWTH Aachen im WS 1993/94 wechselte ich zum SS 1994 an die Georg-August-Universität Göttingen zum Studium der Medizin. Im Rahmen meines Praktischen Jahres leistete ich ein Tertial am Zentralkrankenhaus Bremen-Ost ab (Chirurgie), zwei weitere Tertiale am Centre Hospitalier Universitaire Montpellier, Frankreich (Innere Medizin, Neurologie), sowie am Sunnybrook \& Women's College Health Science Centre, University of Toronto, Kanada (Neurologie). Im Oktober 2000 schloss ich das Medizinstudium mit dem 3. Staatsexamen ab.

Seit dem 1.2.2001 bin ich in der Neurochirurgischen Abteilung (Prof. Dr. med. M. Bettag) am Krankenhaus der Barmherzigen Brüder in Trier als Arzt in Weiterbildung tätig. 